\title{
Herstellung von Optiken für weiche Röntgenstrahlung und deren Charakterisierung an Labor- und Synchrotronstrahlungsquellen
}

\author{
Dissertation zur Erlangung \\ des mathematisch-naturwissenschaftlichen Doktorgrades \\ „Doctor rerum naturalium“ \\ der Georg-August-Universität zu Göttingen
}

\author{
vorgelegt von \\ Michael Reese
}

aus

Hamburg

Göttingen, 2011 

Referent:

Prof. Dr. T. Salditt

Korreferent:

Prof. Dr. G. Marowsky

Tag der Disputation: 



\section{Inhaltsverzeichnis}

$\begin{array}{lr}\text { Einleitung } & 1\end{array}$

1 Grundlagen 5

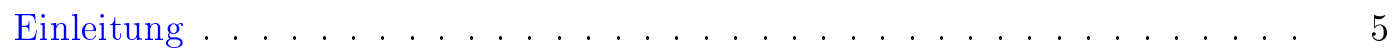

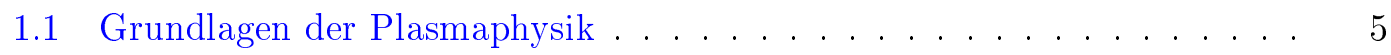

1.1.1 Plasmaprozesse und -eigenschaften . . . . . . . . . . 5

1.1 .2 Plasmaemission . . . . . . . . . . . . . . . . 8

1.2 Extrem ultraviolette Strahlung . . . . . . . . . . . . . . 9

1.2.1 Wechselwirkungen extrem ultravioletter Strahlung mit Materie . 9

1.2 .2 Brillanz . . . . . . . . . . . . . . . . . . 13

1.2 .3 Röntgenröhren . . . . . . . . . . . . . . . . . . 13

1.2.4 Synchrotronquellen . . . . . . . . . . . . . . . . . . . 14

1.2.5 Freie-Elektronenlaser . . . . . . . . . . . . . . . . . 15

1.2 .6 Röntgenlaser . . . . . . . . . . . . . . . . 15

1.2.7 Erzeugung hoher Harmonischer . . . . . . . . . . . . . . . 16

1.2 .8 Gasentladungsquellen . . . . . . . . . . . . . . . . 16

1.2 .9 Laserplasmaquellen . . . . . . . . . . . . . . . . . . . . 16

1.3 Röntgenoptiken . . . . . . . . . . . . . . . . . . . 18

1.3 .1 Blenden . . . . . . . . . . . . . . . . . . 18

1.3.2 Fresnelsche Zonenplatten . . . . . . . . . . . . . . . 19

2 Targetkonzepte und deren Anwendung 23

Einleitung . . . . . . . . . . . . . . . . . . 23

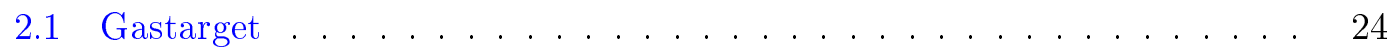

2.2 Flüssiges Argon als Targetmaterial . . . . . . . . . . . . . . . . 28

2.2.1 Aufbau des Argonkryostaten . . . . . . . . . . . . . . 29

2.2.2 Betrieb des Argonkryostaten . . . . . . . . . . . . . . 29

2.2.3 Lagestabilität des Kryostaten und des Argonstrahls . . . . . . . . 36

2.2.4 Debris-Erzeugung während des Betriebes . . . . . . . . . . . 38

2.3 Aufbau des Laborröntgenmikroskops . . . . . . . . . . . . . . . 41

2.3 .1 Design . . . . . . . . . . . . . . . . . . . 41

2.3.2 Erste Detektion von XUV-Strahlung . . . . . . . . . . . . 44 
2.3.3 Justage des Beleuchtungsstrahlgangs . . . . . . . . . . . . . 46

2.3.4 Photonendichte . . . . . . . . . . . . . . . . 47

2.4 Fazit . . . . . . . . . . . . . . . . . . . . . . 49

3 Charakterisierung von Multischicht-Laue-Linsen 51

Einleitung . . . . . . . . . . . . . . . . . . 51

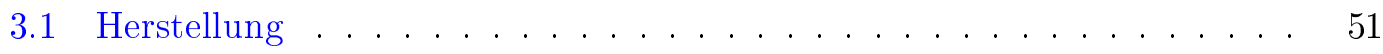

3.1 .1 Motivation ...................... 52

3.1 .2 Pulsed Laser Deposition . . . . . . . . . . . . . . . . . 52

3.1 .3 fokussierter Ionenstrahl _. . . . . . . . . . . . . . . 53

3.1.4 Fertigung der Multischicht-Laue-Linse . . . . . . . . . . . 54

3.2 Experimentelle Strahlcharakterisierung . . . . . . . . . . . . . 58

3.2 .1 Divergenzwinkelexperiment . . . . . . . . . . . . 58

3.2 .2 Schneidblendenrastermessung . . . . . . . . . . . . . . 63

3.2 .3 Messung am Synchrotron . . . . . . . . . . . . . . . . 71

3.2 .4 Farbzentrenerzeugung . . . . . . . . . . . . . 74

3.3 Simulationen . . . . . . . . . . . . . . . . 76

3.3.1 Das Kirchhoffsche Beugungsintegral . . . . . . . . . . . 77

3.3.2 Modellierung des Experiments . . . . . . . . . . . . . . . . 79

3.3.3 Simulationsergebnisse einer Teststruktur . . . . . . . . . . . . 82

3.3.4 Simulationsergebnisse für Multischicht-Laue-Linse 1a . . . . . . . 84

3.3.5 Simulationsergebnisse für Multischicht-Laue-Linse 1b . . . . . . . 91

3.3.6 Simulationsergebnisse für Multischicht-Laue-Linse 2 . . . . . . 92

3.3.7 Simulationsergebnisse für Multischicht-Laue-Linse, Volllinse . . . 92

3.4 Vergleich und Diskussion der Ergebnisse . . . . . . . . . . . . . . . 99

3.4.1 Multischicht-Laue-Linse 1a/b . . . . . . . . . . . . . . . . . 99

3.4.2 Multischicht-Laue-Linse $2 \ldots \ldots$. . . . . . . . . . . . . . 99

3.4.3 Einfluss experimenteller Fehlerquellen . . . . . . . . . . . . . 100

3.4.4 Fazit . . . . . . . . . . . . . . . . . . . . . 101

4 Ultra-kleine Lochblenden 103

Einleitung . . . . . . . . . . . . . . . . . . . . 103

4.1 Herstellung . . . . . . . . . . . . . . . . . . . . . . . 103

4.1 .1 Aufdampfen . . . . . . . . . . . . . . . . . . 104

4.1 .2 fokussierter Ionenstrahl . . . . . . . . . . . . . . . . . 105

4.2 Experimentelle Charakterisierung . . . . . . . . . . . . . . . . 107

4.2.1 Voruntersuchung der ultra-kleinen Lochblenden . . . . . . . . . . 107

4.2.2 Wellenleitende Eigenschaften der ultra-kleinen Lochblenden . . . 110

4.3 Ptychographic Coherent Diffractive Imaging . . . . . . . . . . . . . . 117 
4.3.1 Algorithmus . . . . . . . . . . . . . . . . . . . . . 118

4.3 .2 Versuchsaufbau . . . . . . . . . . . . . . . . . . 119

4.3.3 Ergebnis Rekonstruktion . . . . . . . . . . . . . . . . . . . 119

4.3.4 Chemischer Kontrast in der Ptychographie. . . . . . . . . . 119

4.4 Simulation . . . . . . . . . . . . . . . . . . . . . 123

4.4.1 Das finite-Differenzen Programm Planar Wave Guide . . . . . . . 123

4.4.2 Simulation der ultra-kleinen Lochblenden . . . . . . . . . . . . 123

4.5 Vergleich der Ergebnisse . . . . . . . . . . . . . . . 127

5 Zusammenfassung und Ausblick 129

$\begin{array}{ll}\text { A Ausbreitung eines Gaußschen Strahlenbündels } & 131\end{array}$

$\begin{array}{ll}\text { B Übertragung der Momentenbreite in Halbwertsbreite } & 133\end{array}$

$\begin{array}{ll}\text { C Simulation der Experimente mit Multischicht-Laue-Linsen } & 135\end{array}$

$\begin{array}{ll}\text { D Analyse der Beugungsbilder der ultra-kleinen Lochblenden } & 145\end{array}$

$\begin{array}{ll}\text { Literaturverzeichnis } & 153\end{array}$

$\begin{array}{lr}\text { Danksagung } & 169\end{array}$

$\begin{array}{ll}\text { Lebenslauf } & 171\end{array}$ 


\section{Abbildungsverzeichnis}

0.1 Das elektromagnetische Spektrum . . . . . . . . . . . . . . . 1

0.2 Absorptionslängen im Wasserfenster . . . . . . . . . . . . . 3

1.1 Elektronendichteverlauf eines laserinduzierten Plasmas . . . . . . . . . . 7

1.2 Emissionsspektrum eines heißen und dichten Plasmas . . . . . . . . . 8

1.3 Wechselwirkungsquerschnitt von Kohlenstoff . . . . . . . . . . . . . . 12

1.4 Brillanzen verschiedener Röntgenquellen . . . . . . . . . . . . . . . . . . . . . . 14

1.5 Konstruktion einer Fresnelschen Zonenplatte . . . . . . . . . . . . . . . 20

2.1 Proch-Trickl-Ventil . . . . . . . . . . . . . . . . . . 25

2.2 Spektren der Targetgase . . . . . . . . . . . . . . . . 27

2.3 Spektrum eines Gastarget und eines kryogenen Festkörpertargets . . . . 28

2.4 Schematischer Aufbau des Argonkryostaten . . . . . . . . . . . . . . 31

2.5 Phasendiagramm von Stickstoff und Argon . . . . . . . . . . . . . . . . . . . . 32

2.6 Kryogener Argonstrahl . . . . . . . . . . . . . . . . . . . . 33

2.7 Geometrische Parameter einer beliebigen Düse . . . . . . . . . . . . . 33

2.8 Verschiedene Kapillare im Betrieb. . . . . . . . . . . . . . . . . . . . . 34

2.9 Transmission von XUV-Strahlung im Versuchsaufbau . . . . . . . . . 35

2.10 Lage der Kapillare und des Argonstrahls . . . . . . . . . . . . . . . . 37

2.11 Beschädigung der Kapillare durch Debris . . . . . . . . . . . . . . . . . . . . . . . . . . . . . . . 38

2.12 Betrieb des kryogenen Argonstrahls . . . . . . . . . . . . . . . . . . 39

2.13 Beschädigung von Siliziumnitrid-Membranen durch Schneebälle . . . . . 40

2.14 Versuchsdesign des Laborröntgenmikroskops . . . . . . . . . . . . . . . 43

2.15 Foto des Aufbaus zur Laborröntgenmikroskopie . . . . . . . . . . . . . . . . . . . 43

2.16 Transmission von Ti- und LiF-Filter . . . . . . . . . . . . . . . . . . . . . . . . . 44

2.17 Detektion von XUV Strahlung . . . . . . . . . . . . . . . . 45

2.18 Justage des Beleuchtungsstrahlengangs . . . . . . . . . . . . . . . 46

2.19 Justage des Kondensorspiegels . . . . . . . . . . . . . . . . . . . . . . . . 47

2.20 Messung der Photonendichte an der Probenposition . . . . . . . . . . . 48

3.1 Herstellung Multischicht-Laue-Linse . . . . . . . . . . . . . 56

3.2 Multischicht-Laue-Linse, Probe 1a . . . . . . . . . . . 57

3.3 Aufbau Divergenzwinkelexperiment . . . . . . . . . . . . . 58 
3.4 Detektorbild Divergenzwinkelexperiment . . . . . . . . . . . . 59

3.5 Strahloptische Überlegung zum Divergenzwinkelexperiment . . . . . . . 60

3.6 Im Divergenzwinkelexperiment bestimmte Strahlbreiten . . . . . . . . . 61

3.7 Modellierung der Daten der Schneidblendenrastermessung . . . . . . . . 65

3.8 Aufbau des Schneidblenden-Experiments . . . . . . . . . . . . . . . . 67

3.9 Rohdaten der Schneidblendenrastermessung . . . . . . . . . . . . . 68

3.10 gemessene Strahltaille von Probe $1 b \ldots \ldots$. . . . . . . . . 70

3.11 Strahlprofil an der Strahltaille von Probe 1b . . . . . . . . . . . 70

3.12 Intensitätsverteilung hinter der Multischicht-Laue-Linse 1 (BESSY II) • 71

3.13 Divergenzwinkelbestimmung am BESSY II . . . . . . . . . . . . . . 72

3.14 Vergleich der Strahlfeinstrukturierung . . . . . . . . . . . . . 73

3.15 Laseraktive Farbzentren in LiF-Kristallen . . . . . . . . . . . . . . . . . . . 75

3.16 STED-Mikroskopie der LiF-Farbzentren . . . . . . . . . . . . 76

3.17 Fluoreszenz-Signal in Abhängigkeit der STED-Laser Intensität. . . . . . 77

3.18 Kirchhoffsches Beugungsintegral . . . . . . . . . . . . . . . 78

3.19 Simulationsgrößen des diskretisierten Kirchhoffschen Beugungsintegrals . 81

3.20 Ausführung des diskretisierten Kirchhoff'schen Beugungsintegrals . . . . 81

3.21 Intensitäten des Wellenfelds hinter einem Gitter . . . . . . . . . . . . . 83

3.22 Lage der Beugungsordnungen der Teststruktur . . . . . . . . . . . . . 83

3.23 Kaustik hinter der Multischicht-Laue-Linse 1a . . . . . . . . . . . . . . . 84

3.24 Detailsimulation des Bildpunkts hinter der Multischicht-Laue-Linse 1a . 86

3.25 Strahlbreiten nahe des Bildpunkts bei Probe $1 \ldots \ldots$. . . . . . . . 86

3.26 Vergleich Bildbreite und Intensität bei einer Punktquelle . . . . . . . . . 89

3.27 Vergleich Bildbreite und Intensität einer ausgedehnten Quelle . . . . . . 89

3.28 Strahlkaustik hinter der Multischicht-Laue-Linse 1a (fehlerbehaftet) . . . 90

3.29 Linearer Schichtdickenfehler der Multischicht-Laue-Linse . . . . . . . . . 90

3.30 Strahlkaustik hinter der Multischicht-Laue-Linse 1b . . . . . . . . . . 93

3.31 Strahlkaustik hinter der Multischicht-Laue-Linse 1b - Detail . . . . . . . 93

3.32 Breite des Strahls hinter der Multischicht-Laue-Linse 1b . . . . . . . . . 94

3.33 Bildbreite der Multischicht-Laue-Linse 1b . . . . . . . . . . . . . . 94

3.34 Strahlkaustik hinter einer fehlerhaften Multischicht-Laue-Linse 1b . . . . 95

3.35 Breite des Strahls hinter einer fehlerbehafteten Multischicht-Laue-Linse 1b 95

3.36 Strahltaille hinter der Multischicht-Laue-Linse 1b . . . . . . . . . . . . 96

3.37 Intensitäten des Wellenfelds hinter Probe $2 \ldots \ldots$. . . . . . . 96

3.38 Breite des Strahls hinter der Multischicht-Laue-Linse 2 . . . . . . . . . . 97

3.39 Strahlkaustik hinter einer Vollfeld Multischicht-Laue-Linse . . . . . . . . 97

3.40 Strahlkaustik hinter einer Vollfeld Multischicht-Laue-Linse - Detail . . . 98

3.41 Bildbreite der Vollfeld Multischicht-Laue-Linse . . . . . . . . . . . . . . 98 
4.1 Oberflächenprofil einer Goldfolie . . . . . . . . . . . . . . . . 105

4.2 Herstellung der ultra-kleinen Lochblenden . . . . . . . . . . . . . . . . 106

4.3 Aufbau Vorcharakterisierung von UNHs . . . . . . . . . . . . . . 108

4.4 Einsetzende Wellenleitung bei Lochblenden an einer Laborquelle . . . . 109

4.5 Zusammenstellung ultra-kleiner Lochblenden - Photonenenergie . . . . . 112

4.6 Zusammenstellung ultra-kleiner Lochblenden - Durchmesser . . . . . . . 113

4.7 Auswertung der Beugungsringe hinter einem UNH . . . . . . . . . . . 114

4.8 q-Raum Lage des ersten Beugungsminimums beim $1 \mu \mathrm{m}$ UNH . . . . . . 115

4.9 q-Raum Lage des ersten Beugungsminimums beim $3 \mu \mathrm{m}$ UNH . . . . . . 116

4.10 Resultierende Durchmesser der 1um UNHs . . . . . . . . . . . . . . . 116

4.11 Rekonstruktion des Wellenfeldes am UNH-Ausgang . . . . . . . . . . . . 120

4.12 Rekonstruktion der Objektfunktionen des $\mathrm{SiO}_{2^{-}} / \mathrm{PMMA-Modellsystems} 121$

4.13 Rekonstruktion der Objektfunktionen eines D. radiodurans-Bakteriums . 122

4.14 Simulation ultra-kleiner Lochblenden mit Planar Wave Guide . . . . . . 124

4.15 Simulation der ultra-kleinen Lochblenden des Ptychographie-Experiments 125

4.16 Vergleich der rekonstruierten und simulierten Probenfunktion . . . . . . 126 


\section{Tabellenverzeichnis}

2.1 Parameter der in den Experimenten verwendeten Laser . . . . . . . . . 26

2.2 Photonenfluss der Laserplasmaquelle (kryogenes Argontarget) . . . . . . 48

3.1 In den Experimenten verwendete Multischicht-Laue-Linse . . . . . . . . 55

3.2 Ergebnisse der Divergenzwinkelmessungen, Brennweite . . . . . . . . . 62

3.3 Eigenschaften des Strahls hinter Probe 1b . . . . . . . . . . . . . 69

3.4 Beugungswinkel aus der Divergenzwinkelmessungen . . . . . . . . . . 71

3.5 Ablenkwinkel der Teststruktur . . . . . . . . . . . . . . 82

3.6 Parameter der MLL Simulationen . . . . . . . . . . . . . . . 85

3.7 Aus Simulation und Messungen gewonnene minimale Strahltaillenbreiten 102

4.1 Ultra-kleinen Lochblenden $1470 \mathrm{~nm}$ Folie . . . . . . . . . . . . . . . . . . 111

4.2 Ultra-kleinen Lochblenden $1860 \mathrm{~nm}$ Folie . . . . . . . . . . . . . . . . 111 



\section{Einleitung}

Das Ziel dieser Arbeit ist die Entwicklung und Charakterisierung von verbesserten Quellen und Optiken für weiche Röntgenstrahlung. Mikroskopische Untersuchungen mit Röntgenstrahlung sind in den letzten Jahrzehnten immer bedeutender geworden. Lange Zeit war diese Technologie an die Großfforschungsanlagen zur Synchrotronstrahlungserzeugung gebunden. Laborstrahlungsquellen sind hingegen einer größeren Gemeinschaft von Forschern zugänglich und machen diese gleichzeitig unabhängiger von den Großforschungsanlagen. Wenngleich die Brillanzen dieser Strahlungsquellen hinter der Synchrotronstrahlung zurückbleiben, bieten sie durch ihre permanente Verfügbarkeit eine attraktive Alternative zu den begrenzt zugänglichen und seltenen Großforschungseinrichtungen. Im Folgenden soll eine kurze Motivation und ein Ausblick auf die Arbeit gegeben werden.

Als weiche Röntgenstrahlung bezeichnet man den Spektralbereich von weniger als 5 bis hin zu etwa $0,5 \mathrm{~nm}$ Wellenlänge. Abbildung 0.1 illustriert den Spektralbereich von infraroter (IR) bis zur harten Röntgenstrahlung (XR) und gibt einen Überblick über die Nomenklatur und einige ausgewählte Energien darin.

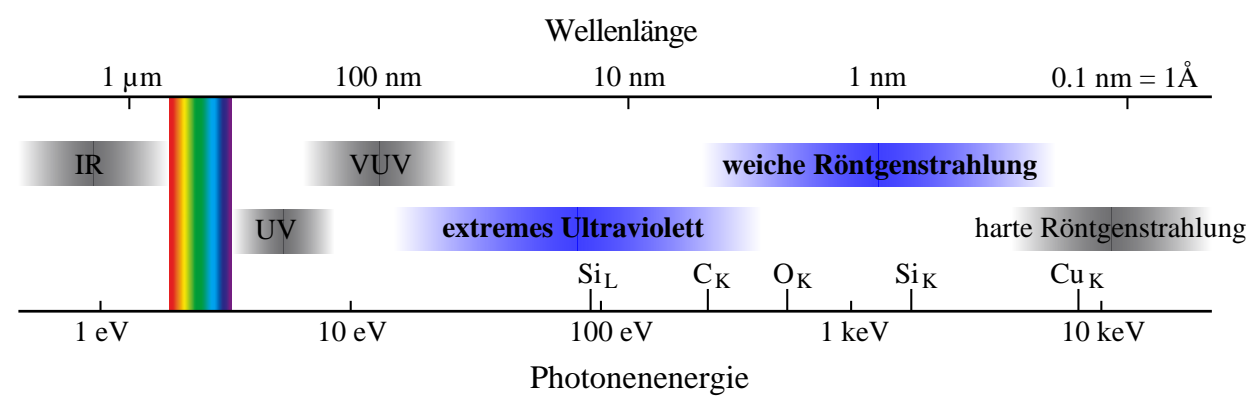

\section{Abbildung 0.1:}

Das elektromagnetische Spektrum vom Infraroten bis hin zur harten Röntgenstrahlung. Unter einer Wellenlänge von $50 \mathrm{~nm}$ spricht man von extrem ultravioletter Strahlung (EUV), deren prominenteste Wellenlänge bei $13 \mathrm{~nm}$ liegt, nämlich der Wellenlänge der Next-Generation-Lithography der Halbleiterindustrie. Die weiche Röntgenstrahlung (SXR - englisch: soft X-ray) oder ebenfalls auch extrem ultraviolette Strahlung (XUV) genannt, schließt unterhalb an und reicht bis etwa $0,5 \mathrm{~nm}$ Wellenlänge. Auf sie folgt die harte Röntgenstrahlung. Innerhalb der weichen Röntgenstrahlung liegt das Wasserfenster, ein Wellenlängenbereich, der besonders interessant für die Untersuchung organischer und biologischer Proben ist. Für einige ausgewählte Elemente sind die Energien der K- und L-Schalen-Absorptionskante dargestellt [Attwood, 1999].

Die Bedeutung der Röntgenstrahlung zur medizinischen Untersuchung wurde bereits 
direkt bei ihrer Entdeckung durch Wilhelm Conrad Röntgen [1896] offenbar. Die einzigartigen physikalischen Eigenschaften ermöglichten bald einen vielseitigen Einsatz bei der Analyse von Materie. Zum einen hat weiche Röntgenstrahlung eine kurze Wellenlänge, was theoretisch Experimente mit hoher räumlicher Auflösung ermöglicht [J. W. Strutt, 3rd Baron Rayleigh, 1879]. Zum anderen durchdringt Röntgenstrahlung optisch dichte Materie und lässt so Beobachtungen in sonst unzugänglichen Bereichen zu. Durch ihre stark energie- und elementabhängigen Absorptions- und Dispersionseigenschaften liefert sie dabei Informationen über die innere Zusammensetzung der Materie. Dabei liegen spezifische Absorptionskanten beinahe aller häufig vorkommenden Elemente in diesem Wellenlängenbereich.

Die Untersuchung organischer und biologischer Proben mit weicher Röntgenstrahlung ist besonders im Bereich des Wasserfensters, das zwischen 2,28 nm und 4,38 nm Wellenlänge liegt, interessant. Eine Auftragung der elementspezifischen Absorptionslängen von Kohlenstoff und Sauerstoff, die das Wasserfenster aufspannen, aber auch einiger anderer Elemente ist in Abbildung 0.2 dargestellt.

Für mikroskopische Untersuchungen benötigt man effektive Optiken für diese Wellenlängen. Aufgrund der starken Absorption der XUV-Strahlung existieren innerhalb einer Absorptionslänge keine effektiven brechenden Optiken. Daher müssen stattdessen Spiegel oder diffraktive Optiken verwendet werden. Deren Herstellung ist mit erheblichem Aufwand verbunden und Gegenstand aktueller Forschung und Entwicklung, wobei die räumliche Auflösung der Röntgenmikroskope immer weiter verbessert werden kann [Sakdinawat und Attwood, 2010].

Für bildgebende und andere Experimente ist die Kohärenz der Strahlung ein zunehmend wichtiges Kriterium. Die räumliche Kohärenz wurde durch die Verbesserung der Synchrotronstrahlungsquellen immer weiter verbessert und kann durch kleinste Aperturen noch weiter erhöht werden. Auf diese Weise wird neben der Intensität auch die Phase der elektromagnetischen Welle als Informationsträger zugänglich.

Die Verfügbarkeit hochwertiger weicher Synchrotronstrahlung war lange auf Speicherringe begrenzt. Durch fortschreitende Entwicklung von Laborquellsystemen gelingt es jedoch zunehmend auch im kleinen Maßstab immer brillantere Strahlung zu generieren, die mittlerweile ein Niveau erreicht, dass Mikroskopie im Wasserfenster möglich macht.

Im Rahmen dieser Arbeit wird in Kapitel 1 ein Überblick über die verwendeten Grundlagen gegeben. Dabei wird die Physik eines laserinduzierten Plasmas zur XUVStrahlungserzeugung eingeführt. Anschließend werden verschiedene Quellsysteme für weiche Röntgenstrahlung vorgestellt und dabei ein Schwerpunkt auf die Laser-PlasmaQuellen gelegt. Danach werden die Prinzipien der hergestellten und charakterisierten Optiken dargestellt.

In Kapitel 2 werden die verwendeten Laborquellen vorgestellt. Zur Erzeugung hochbrillanter Strahlung wird ein Argon-Flüssigtarget benutzt und dessen Betriebsparame- 


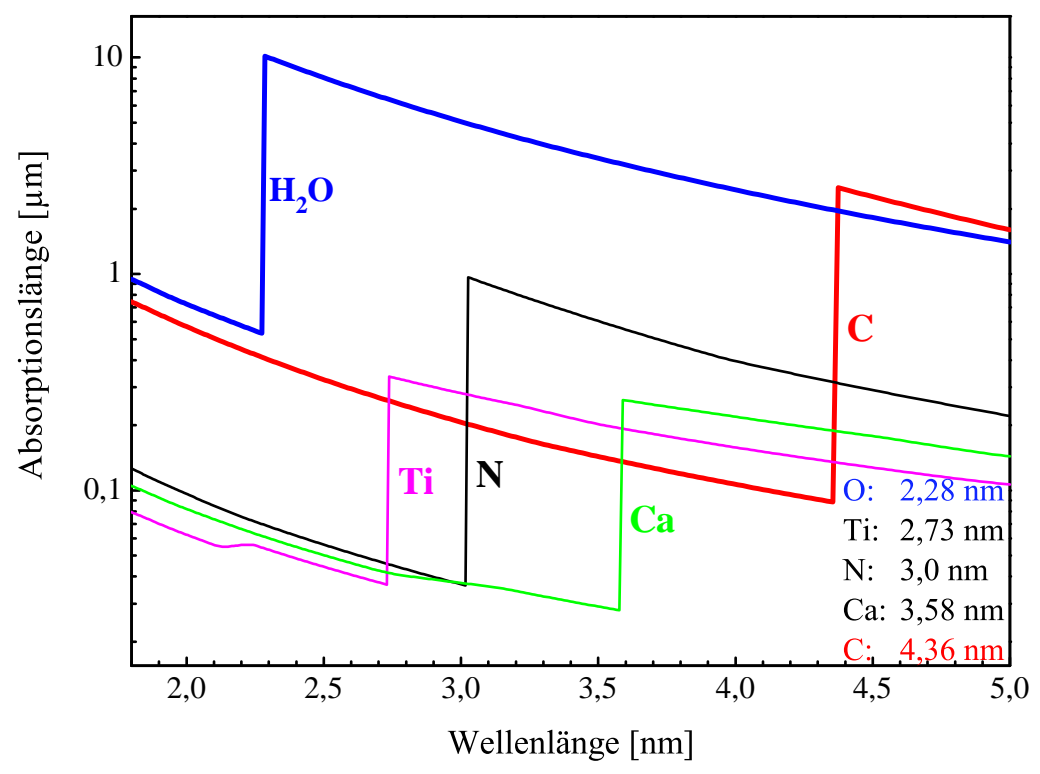

Abbildung 0.2:

Auftragung einiger Absorptionslängen im Wasserfenster. Bei 2,28 nm (oder $543 \mathrm{eV}$ ) wird Sauerstoff, der den Hauptbestandteil der natürlichen wässrigen Umgebung vieler biologischer und organischer Proben bildet, relativ transparent für XUV-Strahlung. Dadurch entsteht ein natürlicher Kontrast zwischen Lösungsmittel und der kohlenstoffhaltigen Probe. Da viele Elemente in diesem Bereich Absorptionskanten besitzen, können beispielsweise Zellen, Proteine, Substanzen aus der Umwelt, wie Bodenproben und andere Systeme, elementensensitiv untersucht werden. Herausgegriffen sind in der Auftragung die Absorptionskanten von Titan, Stickstoff und Kalzium (Daten entnommen aus Henke u.a. [1993]).

ter zur XUV-Strahlungserzeugung untersucht. Anschließend wird die Integration dieser Quelle in ein Experiment zur Röntgenmikroskopie demonstriert. Es wird ein bichromatischer Ansatz verfolgt, der über die Referenzierung zweier mikroskopischer Aufnahmen, einer unter und einer über der spezifischen Absorptionskante des Kalziums eine genaue Lokalisation kalziumhaltiger Strukturen ermöglicht. 
Die Herstellung leistungsfähiger diffraktiver Optiken ist aufgrund der gewünschten Strukturgrößen im Nanometerbereich eine große Herausforderung. Ein neuer Ansatz zur Herstellung von Optiken für weiche Röntgenstrahlung ist der Einsatz sogenannter Multischicht-Laue-Linsen (MLL), deren Herstellung und Charakterisierung an Laborund Synchrotronstrahlungsquellen in Kapitel 3 diskutiert wird. Die aus den Experimenten gewonnenen Strahlparameter einer solchen Linse werden mit Simulationen der Strahlpropagation verglichen.

Die Herstellung und Charakterisierung ultra-kleiner Lochblenden zur Anwendung in der linsenlosen Bildgebung wird in Kapitel 4 gezeigt. In zwei Experimenten am BESSY II Speicherring am Helmholzzentrum in Berlin wurden diese durch Beugungsexperimente und im linsenlosen Bildgebungsverfahren der Ptychographie in ihren Eigenschaften analysiert und mit Simulationen verglichen.

Die Arbeit schließt mit einer Zusammenfassung und einem Ausblick in Kapitel 5. 


\section{Kapitel 1}

\section{Grundlagen}

\section{Einleitung}

In diesem Kapitel wird eine Übersicht der in dieser Arbeit verwendeten Grundlagen gegeben. Dazu wird zunächst eine knappe Zusammenfassung der Plasmaphysik, die für die Beschreibung einer laserinduzierten Plasmaquelle für Röntgenlicht notwendig ist, vorangestellt. Im zweiten Abschnitt folgt ein Überblick über die Eigenschaften von Röntgenstrahlung und die Möglichkeiten ihrer Erzeugung. Anschließend werden die in dieser Arbeit untersuchten Optiken vorgestellt.

\subsection{Grundlagen der Plasmaphysik}

Heiße und dichte Plasmen sind Quellen für extrem ultraviolette Strahlung. In erster Näherung können sie als schwarze Strahler betrachtet werden, deren Strahlungsmaximum durch das Wiensche Verschiebungsgesetz gegeben ist. Damit ein schwarzer Körper im Bereich der weichen Röntgenstrahlung emittiert, muss er eine Temperatur von einigen $100 \mathrm{eV}$ besitzen. Diese Energie liegt deutlich über der Ionisationsenergie aller Elemente, sodass die Quelle im Plasmazustand vorliegen muss. In diesem Kapitel wird übersichtsartig in die Physik von Plasmen durch wichtige Kenngrößen eingeführt.

\subsubsection{Plasmaprozesse und -eigenschaften}

Die Beschreibung eines Plasmas ist ein Vielteilchenproblem, das in seiner vollständigen Beschreibung auf wenige Teilchen beschränkt ist, bei dem alle Geschwindigkeiten, Orte sowie elektrische und magnetische Felder erwogen werden. Alternativ muss die Darstellung mit makroskopischen Parametern vereinfacht werden.

Man kann in einem Plasma zwischen kurzreichweitigen Wechselwirkungen, wie Stoßprozessen und Bremsstrahlung durch Coulombkräfte, und langreichweitigen Wechselwirkungen, wie Plasmaoszillationen, bei denen geladene Plasmateilchen kollektiv an einem Prozess teilnehmen, unterscheiden. 
Ein wichtige Kenngröße für das Abschätzen, ob lang- oder kurzreichweitige Wechselwirkungen dominieren, ist die Debyesche Abschirmlänge $\lambda_{d}$ :

$$
\lambda_{d}=\sqrt{\frac{\epsilon_{0} k_{b} T_{e}}{e^{2} n_{e}}} .
$$

Dabei bezeichnet $\epsilon_{0}$ die elektrische Feldkonstante, $k_{b}$ die Boltzmannkonstante, $T_{e}$ die Elektronentemperatur, $e$ die Elementarladung und $n_{e}$ die Elektronendichte. Für Plasmen zur Erzeugung weicher Röntgenstrahlung, wie sie in dieser Arbeit zum Einsatz kommen, liegt die Debysche Abschirmlänge typischerweise bei etwa $5 \mathrm{~nm}$ [Attwood, 1999]. Innerhalb der Abschirmlänge dominieren Effekte der geladenen Ionen und der freien Elektronen. Bei größeren Längenskalen wird die elektrische Wirkung der Ionen durch die Elektronen abgeschirmt und das Plasma verhält sich elektrisch quasineutral. Im Flüssigkeitsmodell haben die Elektronen die natürliche Eigenfrequenz, mit der das Plasma kollektiv schwingt, die Plasmafrequenz $\omega_{p}$. Es gilt mit $m_{e}$ als der Ruhemasse des Elektrons:

$$
\begin{aligned}
\omega_{p} & =\sqrt{\frac{e^{2} n_{e}}{\epsilon_{0} m_{e}}} \\
& =\frac{1}{\lambda_{d}} \sqrt{\frac{k_{b} T_{e}}{m_{e}}} .
\end{aligned}
$$

Der Brechungsindex eines Plasmas kann aus der Dispersionsrelation für die Ausbreitung elektromagnetischer Wellen in Plasmen als Zusammenhang der Plasmafrequenz $\omega_{p}$ und der sich ausbreitenden Welle der Frequenz $\omega$ angegeben werden [Attwood, 1999].

$$
\begin{aligned}
n & =\sqrt{1-\frac{\omega_{p}^{2}}{\omega^{2}}} \\
& =\sqrt{1-\frac{n_{e}}{n_{c}}}
\end{aligned}
$$

Diese makroskopische Größe ist für laserinduzierte Plasmen von besonderer Bedeutung, da nur für eine Laserfrequenz höher der Plasmafrequenz $\omega>\omega_{p}$ die Strahlung zum Heizen in das Plasma eindringen kann. Andernfalls wird die Strahlung vom Plasma zurück reflektiert. Zusammen mit der Gleichung (1.3) lässt sich die kritische Elektronendichte $n_{c}$ angeben $\left(\omega_{p}=\omega\right)$, bis zu welcher sich die eindringende elektromagnetische Welle noch im Plasma ausbreiten kann:

$$
n_{c}=\frac{\epsilon_{0} m_{e} \omega^{2}}{e^{2}} .
$$




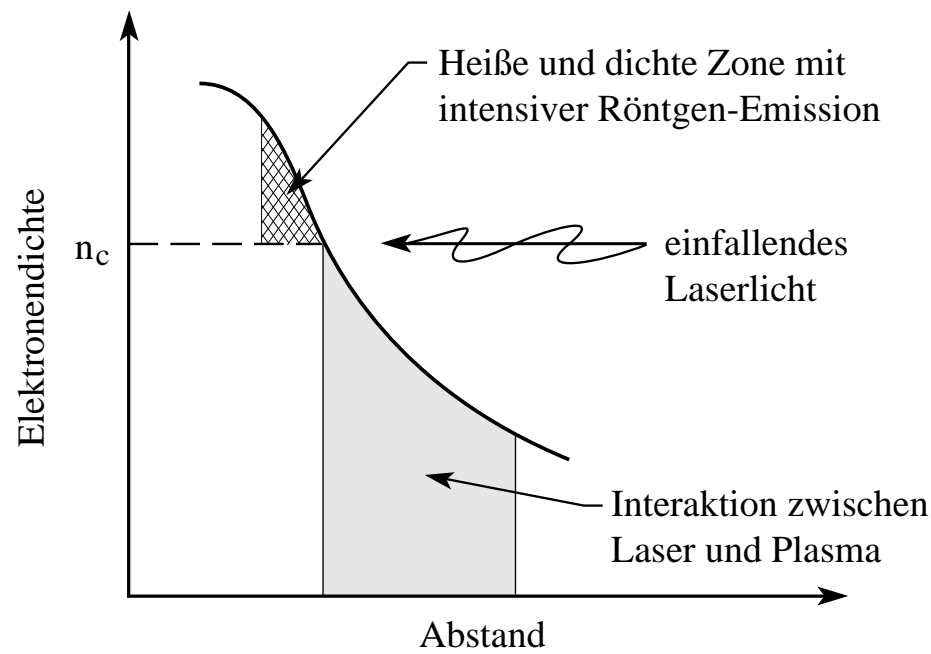

Abbildung 1.1:

Elektronendichteverlauf eines laserinduzierten Plasmas. Das Laserlicht wird besonders effektiv nahe der kritischen Elektronendichte absorbiert. Durch Energietransport in das Innere des Plasmas entsteht ein Bereich intensiver Röntgenemission [Attwood, 1999].

Für die zur Plasmaerzeugung oft verwendeten, gepulsten Nd:YAG-Laser ergibt sich für die fundamentale Wellenlänge von $\lambda=1064 \mathrm{~nm}$ eine kritische Plasmadichte von $10^{21} \frac{\mathrm{e}}{\mathrm{cm}^{3}}$. Wird ein Laserpuls auf ein Target zur Plasmaerzeugung fokussiert, so wird bereits beim Ansteigen der Laserintensität das Targetmaterial ionisiert, wobei Multiphotonen-, Tunnel- und Feldionisation in Abhängigkeit von der eingestrahlten Energie auftreten [Morgan, 1975]. Die weitere Aufheizung des Plasmas geschieht überwiegend bei mittleren Intensitäten $\left(I \leq 10^{15} \mathrm{~W}\right)$ durch den Prozess inverser Bremsstrahlung. Dabei werden die freien Elektronen beschleunigt und ionisieren durch Stöße weitere Targetatome [Kruer, 1988].

Ein Plasma dehnt sich mit hoher Geschwindigkeit aus, wobei die Geschwindigkeit auch von der Masse und dem mittleren Ionisationsgrad der Atome abhängt [Attwood, 1999]:

$$
v_{\text {exp }}=\sqrt{\frac{Z \gamma k_{b} T_{e}}{M}}
$$

$\gamma$ bezeichnet dabei den Adiabatenexponent. Ein zehnfach ionisiertes Argonplasma bei $500 \mathrm{eV}$ expandiert mit etwa 0,14 $\frac{\mu \mathrm{m}}{\mathrm{ps}}$. Diese Expansion führt zu der Ausprägung einer sich selbst regulierenden Heizzone, in der die Strahlung um den Kern des Plasmas nahe der kritischen Elektronendichte effektiv umgesetzt wird (siehe Abbildung 1.1). 


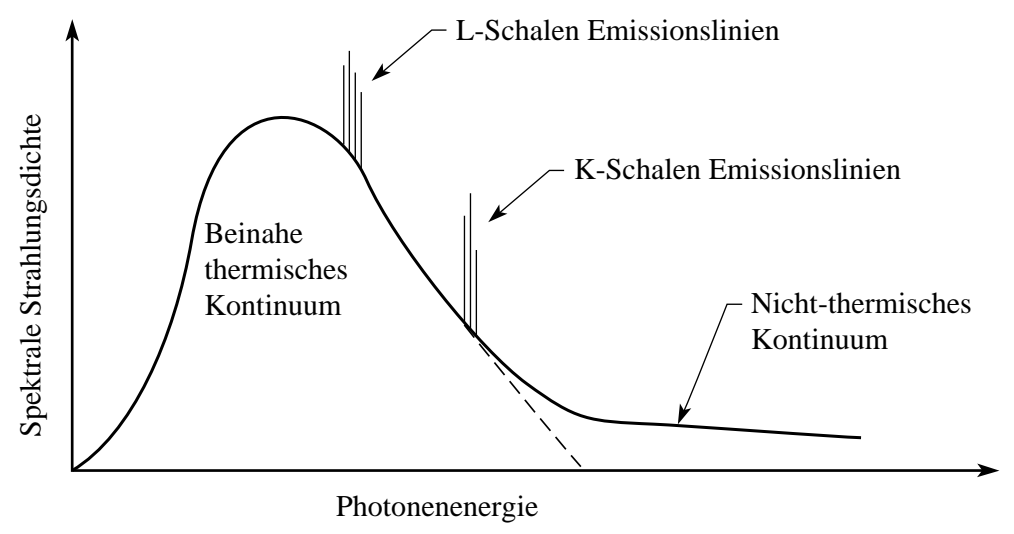

\section{Abbildung 1.2:}

Emissionsspektrum eines heißen und dichten Plasmas. Die Linien beruhen auf verschiedenen lonisierungszuständen der Plasmaatome [Attwood, 1999].

\subsubsection{Plasmaemission}

Ein, wie in Kapitel 1.1.1 beschriebenes Laserplasma, kann in seiner Emissionseigenschaft in erster Näherung als ein Körper mit gleich verteilter Temperatur beschreiben werden, sodass die Abstrahlcharakteristik näherungsweise die eines schwarzen Strahlers ist. Der schwarze Strahler ist ein oberes Limit für die spektrale Leistungsdichte des Plasmas, das sich nicht vollständig im thermischen Gleichgewicht befindet. Darüber hinaus kommt es zu einem nicht-thermischen Kontinuum, in welchem hochenergetische Photonen emittiert werden, die aufgrund von nichtlinearen Elektronenprozessen, wie Resonanzabsorption und stimulierter Raman-Streuung höhere Energiezustände als die Temperatur des Plasmas besitzen [Attwood, 1999; Griem, 2005]. In Abbildung 1.2 ist das Emissionsspektrum eines heißen und dichten Plasmas dargestellt.

Die Emission eines Plasmas setzt sich aus verschiedenen Prozessen des Plasmas zusammen. Zum einen werden freie Elektronen im Coulombfeld der Ionen abgebremst. Dabei entsteht ein breitbandiges Energiespektrum, die Bremsstrahlung, das von der Geschwindigkeitsverteilung und mittleren Energie der Elektronen abhängt. Darüber hinaus können die Elektronen mit den Ionen rekombinieren, wobei auch diese Rekombinationsstrahlung kontinuierlich ist und von der kinetischen Energie der Elektronen abhängt. Die Linienstrahlung wird bei dem Übergang eines in eine höher angeregten Elektrons in eine niedrigeren Energiezustand in Linien emittiert, deren Photonenenergie der Übergangsenergie entspricht. Diese diskreten Energien lassen sich mit dem empirischen Moseleyschen Gesetz berechnen [Mayer-Kuckuk, 1997]:

$$
\frac{1}{\lambda}=R_{\infty}\left(Z-\sigma_{A}\right)^{2}\left(\frac{1}{n^{2}}-\frac{1}{m^{2}}\right) .
$$


Dabei bezeichnet $Z$ die Kernladungszahl, $R_{\infty}$ die Rydbergkonstante, $\sigma_{A}$ die Abschirmkonstante des Kerns durch Elektronen in niedrigeren Schalen und $m$ sowie $n$ die Hauptquantenzahlen des Anfangs- und Endzustandes des Prozesses.

Die spektrale Linienbreite gibt direkten Rückschluss auf Temperatur und Dichte des Plasmas. Ihre Breite hängt dabei von verschiedenen Prozessen innerhalb des Plasmas ab: Von der natürlichen Linienbreite, die durch die Lebensdauer der Niveaus definiert wird, der Dopplerverbreiterung durch thermische Bewegung der Ionen, der Starkverbreiterung, der durch Coulomb-Wechselwirkung induzierten Deformation von Elektronenwolken und der damit verbundenen Verschiebung energetischer Niveaus bei hohen (Ladungs-)Dichten. Darüber hinaus von der Stoßverbreiterung durch Stöße angeregter Ionen, welche die Lebensdauer des angeregten Zustandes verkürzen.

Je nach Ordnungszahl sind diese Prozesse verschieden dominant, sodass bei leichten Elementen vor allem wenige Spektrallinien und praktisch keine Bremsstrahlung emittiert wird. Bei schweren Atomen hingegen führt eine Vielzahl stark verbreiterter Emissionslinien bei einem gleichzeitig hohen Untergrund an Bremsstrahlung zu einem quasi-kontinuierlichen Spektrum.

\subsection{Extrem ultraviolette Strahlung}

In diesem Kapitel wird ein Überblick über die Eigenschaften der Strahlung und der verfügbaren Quellen in diesem Wellenlängenbereich gegeben. Aufgrund der starken Wechselwirkung von extrem ultravioletter (EUV) oder weicher Röntgenstrahlung (XUV/SXR) mit Materie - die freie Weglänge liegt bei wenigen Millimetern unter Normaldruck - kommt sie auf der Erdoberfläche nicht natürlich vor, obwohl die Sonne auch in diesem Spektralbereich ein starker Emitter ist.

Dies stellt auch an die Erzeugung im Labor und am Speicherring die Bedingung, dass die Experimente im Vakuum erfolgen. Die Erzeugung ist dabei auf einer Vielzahl von Wegen möglich. Einige der vorgestellten Methoden sind auf Grofforschungsanlagen angewiesen, während andere auch im Labor realisierbar sind.

\subsubsection{Wechselwirkungen extrem ultravioletter Strahlung mit Materie}

Die Wechselwirkung zwischen weicher Röntgenstrahlung und Materie, sind besonders stark von der Wellenlänge der Strahlung abhängig. Der Absorptionsquerschnitt ist, betrachtet über das ganze elektromagnetische Spektrum, hier beinahe maximal, da die Photonenenergie im Bereich der Bindungsenergien der K- und L-Schalen-Elektronen der Elemente niedriger und mittlerer Ordnungszahl $Z$ liegen [Bearden und Burr, 1967]. Durch diese Eigenschaft, gepaart mit der kurzen Wellenlänge, ist sie für sensitive Untersuchungen bei hoher räumlicher Auflösung sehr geeignet. 


\section{Brechungsindex}

Die Dispersion und Absorption in einem Medium wird durch den komplexen Brechungsindex $n$ gegeben, der im Wellenlängenbereich der Röntgenstrahlung als Abweichung von der Wellenausbreitung im Vakuum angegeben wird:

$$
n=1-\delta+i \beta
$$

Der Realteil des Brechungsindex beschreibt dabei die Brechung und der Imaginärteil die Absorption durch das Medium. Beide sind abseits von Resonanzen deutlich kleiner als eins.

Der atomare, komplexe Streufaktor ( $f$ ) ist bei Vorwärtsstreuung direkt mit dem makroskopischen Brechungsindex verbunden [Attwood, 1999]. Real- und Imaginärteil sind über die Kramers-Kronig Relation verknüpft [Als-Nielsen und McMorrow, 2001].

\section{Reflexion}

Aufgrund der Tatsache, dass der Realteil des Brechungsindex $\operatorname{Re}(n)<1$ ist, kommt es zur totalen äußeren Reflexion von weicher Röntgenstrahlung an Grenzflächen von Vakuum zu Materie. Für spekuläre Reflexion kann der kritische Winkel, unter dem die Strahlung gerade noch total reflektiert wird, aus dem Snelliusschen Brechungsgesetz

$$
n_{\text {Vakuum }} \cdot \cos \left(\theta_{\text {einfallend }}\right)=n_{\text {Materie }} \cdot \cos \left(\theta_{\text {ausfallend }}\right)
$$

abgeleitet werden. Für den Grenzfall, dass der ausfallende Winkel $\theta_{\text {ausfallend }}=0$ wird, ist der einfallende Winkel $\theta_{\text {einfallend }}$ gleich dem kritischem Winkel $\theta_{c}$. Unter Nichtbeachtung der Absorption lässt sich mit $\cos \left(\theta_{c}\right)=1-1 / 2 \cdot \theta_{c}^{2}$ dieser entwickelt als

$$
\theta_{c}=\sqrt{2 \delta}
$$

darstellen. Der kritische Winkel $\theta_{c}$ kann für einige Wellenlänge große Werte von mehreren Grad - mitunter auch deutlich mehr als $10^{\circ}$ - annehmen. Zum Beispiel liegt bei einer Photonenenergie von $92 \mathrm{eV}$ der kritische Winkel von Gold bei 25,7. Aufgrund der hohen Absorption in diffraktiven und brechenden Optiken der weichen Röntgenstrahlung in Medien wird dieser Effekt oft dazu genutzt, die Strahung effektiv zu sammeln und bei geringen Verlusten zu reflektieren. Häufig eingesetzte Optikanordnungen sind die Kirkpatrick-Baez-Spiegel [Kirkpatrick und Baez, 1948], Wolter Teleskope [Wolter, 1952] sowie Mono- und Polykapillare [Bilderback und Thiel, 1995; MacDonald und Gibson, 2003]. Für eine detaillierte Beschreibung der Reflektivität nach den Fresnel-Gleichungen sei an dieser Stelle auf die Darstellung von Attwood [1999] verwiesen.

Die Reflektion von Röntgenstrahlung unter Normaleinfall ist ungleich ineffektiver und 
beträgt oft nur den Bruchteil von Prozenten. Durch das Einsetzen von Vielschichtsystemen kann die Reflektivität jedoch erhöht werden. Dazu werden abwechselnd Materialien einer niedrigen und einer hohen Ordnungszahl $Z$ auf ein Substrat aufgebracht. An jeder Grenzfläche kommt es durch die stark unterschiedlichen Elektronendichten zur Streuung der einfallenden Strahlung, die konstruktiv interferiert und so die Reflektivität für eine bestimmte Wellenlänge und einen zugehörigen Winkel erhöht. Die Dicke der Schichten muss also so gewählt sein, dass es zu der notwendigen Phasenanpassung kommt. Bei senkrechtem Einfall muss diese $\lambda / 2$ betragen. Das bedeutet für die Einzelschicht eine Dicke von $\lambda / 4$. Soll eine Reflektivität unter einem bestimmten Winkel erzielt werden, so ergibt sich die optimale Periode der Schichten aus der Bragg-Bedingung [Attwood, 1999].

Die Forderung von Schichtdicken im Nanometerbereich stellt extrem hohe Anforderungen an den Herstellungsprozess. Die Schichtsysteme müssen nicht nur eine hohe theoretische Reflektivität aufweisen, sondern auch eine Dicke im Bereich von etwa $1 \mathrm{~nm}$ haben. Dieses entspricht nur wenigen Atomlagen. Im Energiebereich des Wasserfensters (284,1 - 543,1 eV) haben Chrom/Scandium und Eisen/Scandium-Schichtsysteme bei Photonenenergien von 120 - $399 \mathrm{eV}$ die höchste erwartete Reflektivität von etwa $50 \%$ unter Normaleinfall [Schäfers u.a., 1998]. Aufgrund der Rauigkeiten der Schichten, die beispielsweise bei Beschichtung oder später durch Interdiffusion entstehen, sind jedoch die erreichten Reflektivitäten deutlich geringer. Dazu siehe beispielsweise:

- 7\% Reflektivität bei $395 \mathrm{eV}$ und senkrechtem Einfall [Schäfers u. a., 1998],

- 5,5\% Reflektivität bei $367 \mathrm{eV}$ und Einfall unter $76^{\circ}$ [Birch u. a., 2003],

- 11,5\% Reflektivität bei $398.6 \mathrm{eV}$ und Einfall unter $80^{\circ}$ [Ghafoor u. a., 2008].

Aufgrund ihrer kleinen spektralen Bandbreite eignen sich diese Spiegel auch als Monochromatoren.

\section{Absorption}

Die stärkste Wechselwirkung zwischen weicher Röntgenstrahlung und Materie ist die Absorption. Aus der geringen Reflexion der Strahlung oberhalb des kritischen Winkels ergibt sich, dass ein Großteil der Strahlung in das Medium eindringt und absorbiert wird. Dieser Prozess setzt sich aus verschiedenen Komponenten zusammen, dem photoelektrischen Effekt sowie der kohärenten und inkohärenten Streuung, wobei letztere bei der weichen Röntgenstrahlung kaum eine Rolle spielt. In Abbildung 1.3 werden die Beiträge der einzelnen Prozesse zum Gesamtwirkungsquerschnitt beispielhaft für Kohlenstoff aufgetragen. Quantitativ beschreibt das Lambert-Beersche Gesetz die Absorption über 


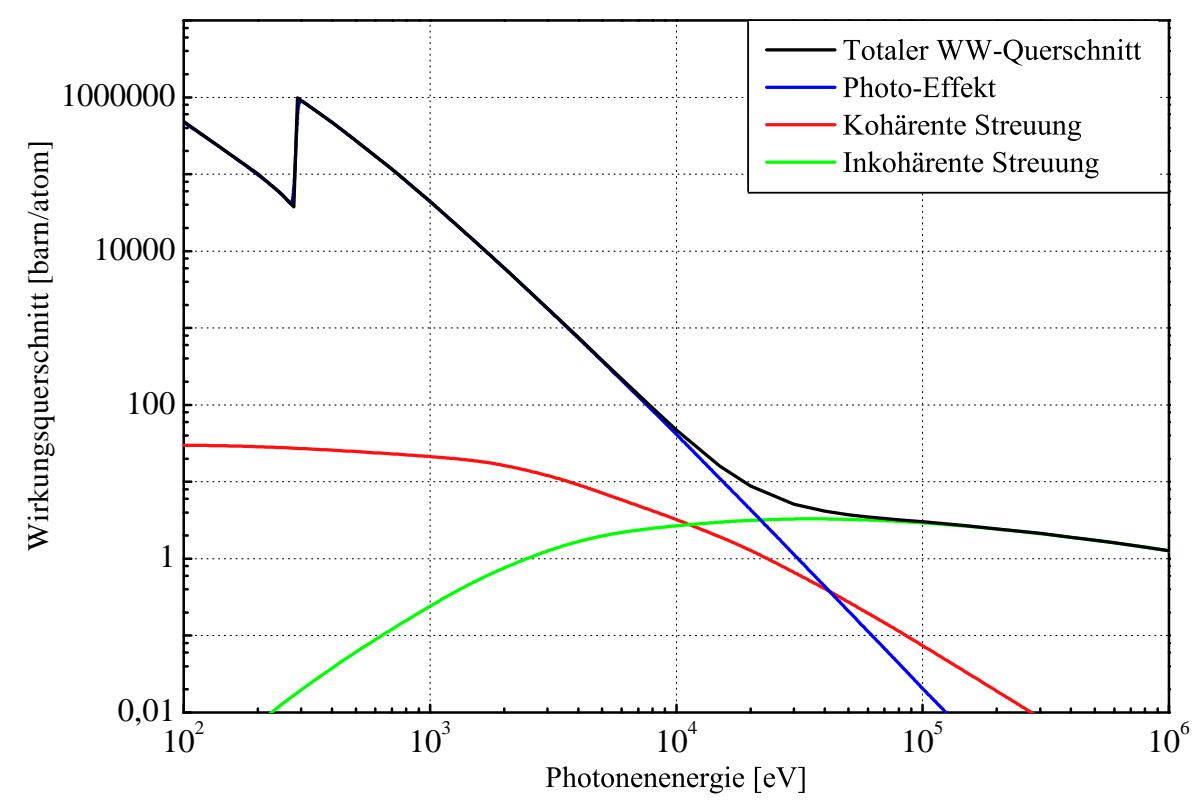

Abbildung 1.3:

Wechselwirkungsquerschnitt von Kohlenstoff $(Z=6)$. Für niedrige Photonenenergien dominiert der Photoeffekt als Wechselwirkungsursache. Kohärente Streuung wird erst zu höheren Energien relevanter. Inkohärente Streuung dominiert die Wechselwirkung hochenergetischer Strahlung (Daten aus Henke u.a. [1993]; Berger u. a. [2010]).

den Absorptionskoeffizienten $\mu$ und die Eindringtiefe $z$ :

$$
I(z)=I_{0} \cdot e^{-\mu z}
$$

Es besteht ein direkter Zusammenhang zwischen dem Absorptionskoeffizient $\mu$ und dem Wechselwirkungsquerschnitt das Atoms [Als-Nielsen und McMorrow, 2001]:

$$
\begin{aligned}
\mu & =n_{a} \sigma_{a} \\
& =\left(\frac{\rho_{m} N_{A}}{A}\right) \sigma_{a} .
\end{aligned}
$$

Dabei bezeichnet $n_{a}$ die atomare Dichte, $\rho_{m}$ die Massendichte, $N_{A}$ die Avogadro-Zahl und $A$ die atomare Massenzahl. Zum makroskopischen Imaginärteil des Brechungsindex besteht folgender Zusammenhang [Attwood, 1999]:

$$
\beta=\frac{\lambda}{2 \pi} \frac{\mu}{2} .
$$


Bei senkrechtem Einfall in ein Medium sind für weiche Röntgenstrahlung im Wasserfenster typische Eindringtiefen für Festkörper, bei denen die Intensität auf 1/e abgefallen ist, im Bereich unter $100 \mathrm{~nm}$. Für Gold sind dies $37 \mathrm{~nm}$ und für Silizium $29 \mathrm{~nm}$ bei $2,88 \mathrm{~nm}$ Wellenlänge [Henke u. a., 1993]. Obwohl die Absorptionslängen für Gase aufgrund der geringeren Atomdichte größer sind, sind sie dennoch fast immer im Bereich von einigen hundert Mikrometern. Um die Strahlung im Experiment nutzen zu können, muss es daher unter ausreichend gutem Vakuum durchgeführt werden.

\subsubsection{Brillanz}

Eine Kennzahl, um die vielen verschiedenen Röntgenquellen miteinander zu vergleichen ist die Brillanz. Durch sie erreicht man eine Vergleichbarkeit der Strahlungsquellen, die sich mitunter erheblich in ihren Abstrahlungseigenschaften wie Wellenlänge und deren Spektrum, Divergenz, Kohärenz und Pulsdauer unterscheiden. Die Brillanz ist definiert als die Anzahl der Photonen pro Sekunde bezogen auf den Raumwinkel, die Quellfäche und die spektrale Bandweite [Als-Nielsen und McMorrow, 2001]:

$$
\text { Brillanz }=\frac{\text { Photonen/Sekunde }}{\text { Raumwinkel }\left[\mathrm{mrad}^{2}\right] \cdot \text { Quellfläche }\left[\mathrm{mm}^{2}\right] \cdot \text { Bandweite }[0,1 \%]}
$$

Die erreichten Brillanzen verschiedener Röntgenquellen in Abhängigkeit der Photonenenergie sind in Abbildung 1.4 aufgetragen. Einerseits werden die Spitzenbrillanzen (Abbildung 1.4(a)), die die Brillanzen bezogen auf einen Puls angeben, andererseits die mittlere Brillanzen (Abbildung 1.4(b)), die jene über einen längeren Betrieb der Quelle angeben, dargestellt.

\subsubsection{Röntgenröhren}

Röntgenröhren haben sich seit der Entdeckung der Röntgenstrahlung durch W. C. Röntgen in ihrem grundsätzlichen Aufbau kaum verändert [Röntgen, 1896]. Eine Röntgenröhre besteht aus einer Glühkathode, die Elektronen freisetzt, welche anschließend durch ein elektrisches Feld zu einer Anode hin beschleunigt werden. Die Elektronen treten beim Auftreffen auf die Anode in heftige Wechselwirkung mit den Elektronenschalen der Atome des Anodenmaterials und werden dabei stark abgebremst. Dadurch entsteht die sogenannte Bremsstrahlung, welche durch elementspezifische charakteristische Emissionslinien ergänzt wird, die durch Ionisations- und Rekombinationsvorgänge innerer Schalen hervorgerufen werden. Zur Steigerung der Leistung von Röntgenröhren, die im Wesentlichen nur durch die aufnehmbare thermische Last der Anode begrenzt wird, wurden Rotationsanoden und flüssige Anoden eingeführt. Eine Röntgenröhre strahlt kontinuierlich, daher wird für sie keine Spitzenbrillanz angegeben. Weil die Effizienz der 


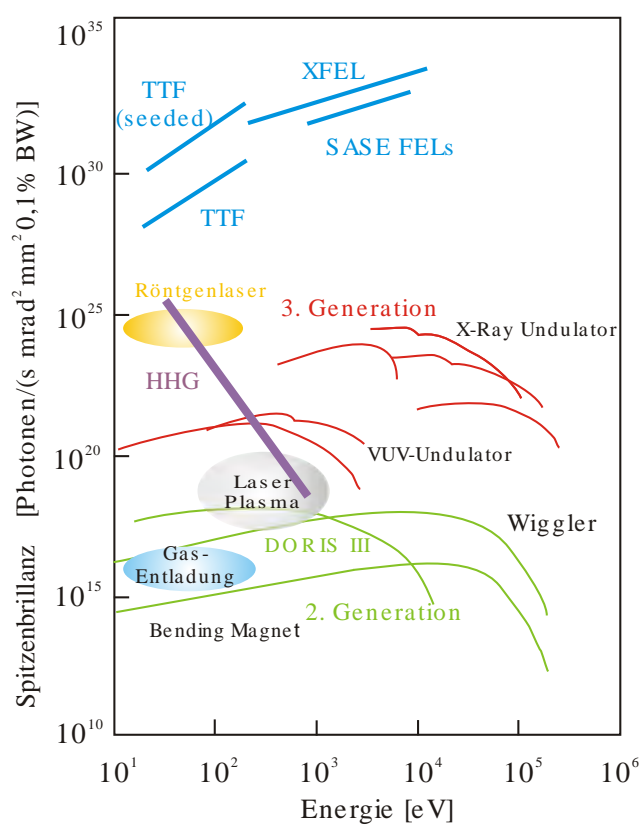

(a) Spitzenbrillanz

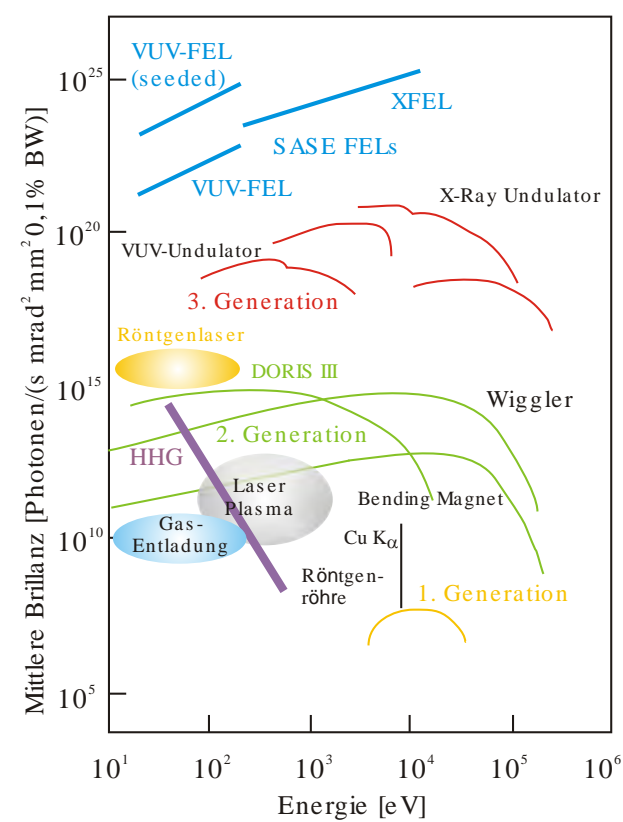

(b) mittlere Brillanz

Abbildung 1.4:

Vergleich verschiedener Röntgenquellen in ihrer Spitzen- (a) und ihrer mittleren Brillanz (b). Die höchsten Brillanzen erreicht man zur Zeit mit den Freie-Elektronenlasern [Peth, 2008].

Röntgenröhre proportional zur Beschleunigungsspannung ist, findet sie für die niederenergetische weiche Röntgenstrahlung bisher aufgrund ihrer geringen Effizienz wenig Anwendung [Krieger, 2001].

Skoglund u. a. [2010] jedoch zeigen eine hochbrillante Labor-Röntgenquelle auf Basis einer Wasserjet Anode, die Sauerstoff-Linienemission bei 2,36 nm Wellenlänge erzeugt (Helligkeit von $3 \cdot 10^{9} \frac{\text { Photonen }}{s \cdot \mu \mathrm{m}^{2} \cdot \text { sr.Linienbreite }}$ ) und erfolgreich in ein Labormikroskop integriert wurde.

\subsubsection{Synchrotronquellen}

In Synchrotron- und Speicherring-Einrichtungen werden leichte, geladene Elementarteilchen auf relativistische Geschwindigkeiten beschleunigt und durch Magnete auf eine geschlossene Bahn gezwungen. Durch die erzwungene Richtungsänderung strahlen die bewegten Teilchen die sogenannte Synchrotronstrahlung ab (siehe beispielsweise [Kim, 1989; Als-Nielsen und McMorrow, 2001]).

Nach der Reihenfolge ihres Auftretens unterscheidet man grob drei Arten von Synchrotronstrahlung. Die Quellen erster Generation sind Ablenkmagnete, die den Strahl auf einen geschlossenen Orbit zwingen. Die Änderung der Bewegungsrichtung führt zum 
Entstehen der Strahlung. Bei den Quellen der zweiten Generation befinden sich in den geraden Passagen der Teilchenstrahlführung alternierende Magnetfelder, die Wiggler, die die geladenen Teilchen auf eine oszillierende Bahn zwingen, wobei sich die Amplituden der entstehenden Strahlung jeder Oszillation inkohärent addieren. Die dritte Generation der Synchrotronstrahlquellen sind Undulatoren, die wie Wiggler alternierende Magnetfelder erzeugen, die jedoch eine geringere Feldstärke und eine höhere Periodenzahl haben. Die Amplituden der abgestrahlten Synchrotronstrahlung sind phasengleich und addieren sich mit der der nächsten Oszillation des geladenen Teilchens. So wird nur die selektierte Wellenlänge (und ihre Harmonischen) kohärent verstärkt. Die Strahlung eines Undulators ist quasi monochromatisch, extrem brillant und im gesamten Röntgenspektralbereich verfügbar. In Abbildung 1.4 sind die Brillanzen einiger Synchrotronquellen aufgeführt.

\subsubsection{Freie-Elektronenlaser}

Der Freie-Elektronenlaser (FEL) basiert auf den im Synchrotron der dritten Generation gewonnen Erkenntnissen. Es kommt ein linearer Teilchenbeschleuniger zum Einsatz und der vom Elektronenpaket durchlaufene Undulator ist besonders lang. Durch den „SASEEffect" (SASE, englisch: Self Amplified Stimulated Emission - Selbstverstärkte angeregte Emission) kommt es darin zu Wechselwirkungen zwischen den Elektronen untereinander, die kohärent miteinander in Mikropaketen zu schwingen beginnen [Derbenev u. a., 1982; Murphy und Pellegrini, 1985]. Die so erzeugte Strahlung ist noch brillanter als die eines Undulators und kann mittlerweile bis in den Bereich des Wasserfensters hinunter erzeugt werden [Ackermann u. a., 2007; Faatz u. a., 2011](vergleiche Abbildung 1.4).

\subsubsection{Röntgenlaser}

Aufgrund der geringen Reflektivität von Röntgenstrahlen ist es schwierig, einen Röntgenlaser zu konstruieren, der das Prinzip des Lasers im optisch Sichtbaren nur überträgt. Da die notwendigen Energien des laseraktiven Mediums nicht durch Ionisationszustände der äußeren Schale erreicht werden können, sondern höhere Ionisationsgrade erfordern, kommen Plasmen zum Einsatz, die durch einen Laser oder eine schnelle Gasentladung erzeugt werden. Die kurze Lebensdauer eines Plasmas als laseraktives Medium erzwingt einen kurzen Resonator, der aber räumlich getrennt oder widerstandsfähig gegenüber dem Plasma sein müsste. Darüber hinaus wären die Reflektivitäten der Resonatorspiegel unter Normaleinfall sehr niedrig. Der Strahl wird daher im Normalfall im Plasma durch Amplified Spontaneous Emission bei einem einzigen Durchlauf erzeugt [Janulewicz u. a., 2004].

Besonders hohe Spitzenbrillanzen bei $32,6 \mathrm{~nm}$ von $2 \cdot 10^{26} \frac{\text { Photonen }}{\mathrm{s} \cdot \mathrm{mm}^{2} \cdot \mathrm{mrad}^{2} \cdot 0,01 \% \mathrm{BW}}$, die auch durch die extrem kurzen Pulse bedingt sind, sind mit Titanfestkörpertargets er- 
reicht worden [Wang u. a., 2006]. Lange Arbeitszeiträume konnten bei Röntgenlasern auf Basis von Plasmen in Gastargets demonstriert werden, da das Targetmaterial permanent nachgeführt werden kann [Fiedorowicz u. a., 2001]. Noch sind diese Quellen jedoch recht experimentell und für Anwendungen nur eingeschränkt nutzbar (beispielsweise Guilbaud u. a. [2010]).

\subsubsection{Erzeugung hoher Harmonischer}

Durch nichtlineare Prozesse hochenergetischer Laserpulse in Materie können hohe Harmonische der eingestrahlten Laserstrahlung anschwingen. In einem vereinfachten Bild wird dabei eine bestimmte Anzahl Photonen absorbiert und ein einzelnes Photon mit all ihrer Energie reemittiert. Ein anderer Erklärungsansatz ist das ,,Simple-Man-Model“, wie es Corkum [1993] beschreibt. Durch das starke elektromagnetische Feld eines Laserpulses wird das elektrische Feld, welches die Elektronen an ihre Kerne bindet, geschwächt, sodass durch Tunnel- und Feldionisation die Atome ionisiert werden. In diesem Feld werden die Elektronen vom Atom weg- und anschließend wieder zum Ion zurück beschleunigt, wobei sie kinetische Energie aufnehmen. Bei der Rekombination mit dem Ion kommt es zur Aussendung von Röntgenstrahlung.

Durch das Fokussieren von Femtosekundenlasern in einem Gas können Röntgenstrahlen über einen großen Spektralbereich erzeugt werden (siehe beispielsweise [Sommerer u. a., 1998; Seres u.a., 2005]). Hohe-Harmonische Quellen wurden bei $29 \mathrm{~nm}$ Wellenlänge erfolgreich zur diffraktiven Bildgebung eingesetzt. Die dabei erreichte räumliche Auflösung der kompakten Anlage liegt bei $214 \mathrm{~nm}$ [Sandberg u. a., 2007].

\subsubsection{Gasentladungsquellen}

Mittels elektrischer Gasentladung, wie beispielsweise durch einen Blitz, kann sehr effektiv ein Plasma erzeugt werden. Unter Ausnutzung des Pinch-Effektes [Bennett, 1934] kann das so erzeugte, gewöhnlich kalte, Plasma durch das azimutale Eigenmagnetfeld des axialen Entladungsstroms zu einem zylinderförmigen heißen und dichten Plasma komprimiert werden.

Gasentladungsquellen sollen vor allem bei Strahlungsquellen der EUV-Lithographie, aber auch in der Erzeugung von Strahlung im Wasserfenster [Bergmann u. a., 2008] Anwendung finden. Diese Quelle mit einer Brillanz von $4,3 \cdot 10^{9} \frac{\text { Photonen }}{\mu \mathrm{m}^{2} \cdot \mathrm{sr} \cdot \mathrm{s}}$ bei $2,88 \mathrm{~nm}$ wurde erfolgreich zur mikroskopischen Bildgebung eingesetzt [Benk u. a., 2008].

\subsubsection{Laserplasmaquellen}

Eine weitere Methode ein heißes und dichtes Plasma zu zünden, ist das Fokussieren eines hochenergetischen Laserspulses auf ein Targetmaterial. Multiphotonenabsorption, 
Tunnel- und Feldionisation [Morgan, 1975] ermöglichen eine hohe Ionisation des Materials, wenngleich die Photonenenergie des eingestrahlten Laserlichts vergleichsweise gering ist. Der Energiebereich, in dem das Plasma emittiert, kann durch die Intensität und Pulsdauer des Laserfokus sowie durch die Wahl des Targetmaterials in einem großen Bereich skaliert werden. Die notwendigen hohen Pulsenergien weniger Nanosekunden Länge werden gewöhnlich mit Nd:YAG-Laser erreicht [Kruer, 1988].

Die Wahl des Targetmaterials erfolgt sowohl nach den Anforderungen der Abstrahlcharakteristik als auch nach experimentellen Anforderungen wie der Repetitionsfrequenz, der geforderten Brillanz, der Zuführbarkeit unter Vakuumbedingungen und der Sauberkeit der Quelle (Erzeugung von Debris, englisch: Debris - Trümmer). Je nach Aggregatzustand unterscheidet man zwischen drei verschiedenen Targetarten.

Bei einem Gastarget wird Gas unter hohem Druck gepulst oder kontinuierlich durch eine Düse als gerichteter Strahl in das Vakuum geleitet. Durch geeignete Verfahren lässt sich die Stärke der Expansion des Gasstrahls in das Vakuum verzögern, sodass ein Plasma effektiv gezündet werden kann. Obwohl es ein sehr sauberes und störungsarmes Target ist, sind durch die geringe Teilchen- und damit Elektronendichte die erreichbaren Brillanzen relativ gering. Wachulak u. a. [2010] zeigen ein EUV-Argon-Gastarget mit einem Photonenfluss von $8,8 \cdot 10^{10} \frac{\text { Photonen in } 4 \pi}{\text { Puls }}$ bei $13,8 \mathrm{~nm}$ Wellenlänge, das Leistungsdichten erzeugt, die für lithographische Experimente ausreichen, bei denen weniger die Brillanz als mehr der Photonenfluss entscheidend ist. Peth [2008] demonstriert ein Gastarget und dessen Charakterisierung mit verschiedenen Targetgasen, so beispielsweise Stickstoff als schmalbandigen Emitter im Wasserfenster mit einem Photonenfluss von $5 \cdot 10^{11} \frac{\text { Photonen }}{\text { Puls s.s }}$ bei $2,88 \mathrm{~nm}$ Wellenlänge.

Die am Laserlabor entwickelten Laserplasmaquellen auf Basis eines Proch-TricklVentils erzeugen bei einem Stickstofftarget einen Photonenfluss von $5 \cdot 10^{12} \frac{\text { Photonen }}{\text { Puls.sr }}$. Das entspricht auf die Quellfläche von $0,071 \mathrm{~mm}^{2}$ bezogen einer Spitzenbrillanz von $8,8 \cdot 10^{15} \frac{\text { Photonen }}{\mathrm{s} \cdot \mathrm{mrad}^{2} \cdot \mathrm{mm}^{2} \cdot \text { Linienbreite }}$. Es wurden Repetitionsraten bis zu $30 \mathrm{~Hz}$ realisiert.

Eine Erhöhung der Teilchen- und Elektronendichte lässt sich durch die Verwendung von Flüssigtargets realisieren. Bei ihnen wird eine Flüssigkeit über eine Düse als Strahl oder als Tropfen in die Vakuumkammer geführt, in der anschließend das Laserplasma gezündet wird. Solche Targets führen zu deutlich brillanteren Quellen als die Gastargets und können wegen der hohen Fließgeschwindigkeit des Targets (bis zu $100 \frac{\mathrm{m}}{\mathrm{s}}$ ) bei hohen Repetitionsraten betrieben werden. Wird das gesamte Targetmaterial in das Plasma überführt, spricht man von einem massenlimitierten Target, bei dem die Erzeugung von Debris minimiert ist. Eine Überführung des Targetmaterials in die flüssige Phase kann allerdings experimentell herausfordernd sein und starke Kühlung (sogenannte Kryotargets), Heizung oder Verflüssigung in einer Lösung erfordern.

Mit Festkörpertargets lassen sich die höchsten Konversionseffizienzen und Brillanzen erzeugen. Bei einer Plasmazündung wird das Targetmaterial ablatiert, sodass zum einen 
Debris in der Vakuumkammer freigesezt wird und zum anderen ersetzt werden muss. Zumeist kommen dabei ein Band oder eine Folie, sogenannte Tape-Targets, oder ein rotierender Zylinder zum Einsatz. Hohe Repetitionsraten verbrauchen entsprechend viel Targetmaterial, sodass diese nur schwer realisierbar sind. Eine Möglichkeit, ein sich regenerierendes Festkörpertarget herzustellen, ist ein Gas oder eine Flüssigkeit auf einem Festkörper kontinuierlich auszufrieren [Amano u. a., 2010]. Die Kondensationsrate dominiert dann die mögliche Repetitionsrate.

\subsection{Röntgenoptiken}

Die Herstellung effektiver brechender Optiken ist im Wellenlängenbereich der weichen Röntgenstrahlung innerhalb einer Absorptionslänge nicht realisierbar, da das Verhältnis des Real- und Imaginärteils des Brechungsindex ausreichend nahe 1 ist [Attwood, 1999]. Dies erfordert alternative Strahlformungsmethoden, die die Beugung oder Reflexion ausnutzen. Die in dieser Arbeit untersuchten, für bildgebende Verfahren mit weicher Röntgenstrahlung wichtigen optischen Elemente werden übersichtsartig vorgestellt.

\subsubsection{Blenden}

Eine Blende im klassischen Sinn ist eine Maske, bei der das einstrahlende elektromagnetische Feld durch eine Maske transmittiert und ansonsten vollständig absorbiert wird. Das austretende elektrische Feld ist praktisch identisch mit dem eintretenden. Diese Art von Blenden, wie Spalte und Lochblenden, sind in der Literatur ausgiebig beschrieben (siehe beispielsweise [Born und Wolf, 1997]). Insbesondere ist die Intensitätsverteilung des Fernfelds hinter einer Lochblende gegeben durch

$$
I(r)=I_{0} \cdot\left(\frac{J_{1}(2 \pi r)}{\pi r}\right)^{2} .
$$

Dabei bezeichnet $I_{0}$ die eingestrahlte Intensität der Strahlung, $r$ den Abstand zur optischen Achse und $J_{1}$ ist die Besselfunktion erster Art. Die Anforderung nach immer kleineren Blenden für Röntgenmikroskopie und Röntgenlithographie, die aufgrund der notwendigen Absorption eine endliche Dicke haben müssen, erhöht das Aspektverhältnis von Durchmesser zu Tiefe. Es ist anzunehmen, dass das optische Verhalten der Blende durch Eigenschaften beeinflusst wird, wie man sie bereits aus der Betrachtung unendlich langer Wellenleiter kennt [Fuhse und Salditt, 2006b]. Eine geschlossene analytische Lösung für sichtbares Licht und Aperturen kleiner der Wellenlänge wurde durch Bethe [1944] gegeben. Numerische Simulationen von sehr dünnen, wenige Wellenlängen dicken und breiten, ultra-kleinen Blenden für EUV-Lithographie wurden von Goldberg u. a. 
[1996] durchgeführt. Für die mit weicher Röntgenstrahlung untersuchten ultra-kleinen Blenden mit einem Durchmesser $d$ deutlich größer als der Wellenlänge $\lambda$ erwartet man ebenfalls ein von der klassischen Lochblende abweichendes Verhalten, das mit abnehmendem Durchmesser immer stärker ausgeprägt ist.

Bei einer Simulation des erwarteten Verhaltens kann der hohe numerische Aufwand die Wellengleichung zu berechnen durch Approximation mit der parabolischen Wellengleichung deutlich reduziert werden [Kopylov u. a., 1995, 1996]. Es gibt einen Übergang zu einem Wellenleiter, der sich in drei Abschnitte einteilen lässt [Fuhse und Salditt, 2006b]:

- Bei einem Verhältnis von Durchmesser $d$ zu Tiefe $l$, das verglichen mit dem kritischen Winkel groß ist $\left(d / l \gg \theta_{c}\right)$, kann das Feld bis auf kleinere Störungen am Rand durch das einfallende Feld beschrieben werden. Das Fernfeld wird gut durch die klassische Beschreibung der Beugung wieder gegeben.

- Ist das Verhältnis kleiner als der kritische Winkel $d / l<\theta_{c}$, kommt es zu wellenleitenden Effekten innerhalb der Blende, bei der die an einem Rand unter dem kritischen Winkel gebrochene Welle den gegenüberliegenden anderen Rand trifft und sich eine aus der Wellenleitung bekannte Modenstruktur auszuprägen beginnt.

- Für einen Durchmesser im Bereich der sogenannten kritischen Weite, die für eine Transmission der Strahlung erforderlich ist [Bergemann u. a., 2003], kommt es zur Ausprägung eines Wellenfeldes, das von der einzelnen, fundamentalen Schwingungsmode dominiert wird. Die Strahlung wird mit kleiner werdendem Durchmesser zunehmend stark gedämpft.

Die Erzeugung kleinster Aperturen für weiche Röntgenstrahlung wird zum jetzigen Zeitpunkt durch die durch einen fokussierten Ionenstrahl geschaffene Strukturen realisiert. Abhängig vom Aspektverhältnis können Lochblenden eines Durchmessers von $200 \mathrm{~nm}$ in $700 \mathrm{~nm}$ Goldfolie realisiert werden [Eisebitt u. a., 2004; Barth u. a., 2010]. Bedingt durch den Strukturierungsprozess sind in dickeren Filmen von etwa 1,5 $\mu \mathrm{m}$ die kleinsten erreichbaren Lochgrößen etwa $400 \mathrm{~nm}$ und werden in dieser Arbeit vorgestellt.

\subsubsection{Fresnelsche Zonenplatten}

Bei Wellenlängen im Bereich von 1 bis $5 \mathrm{~nm}$, also dem Bereich des Wasserfensters, haben sich diffraktive Optiken, wie Fresnelsche Zonenplatten, für Abbildungen mit hoher Auflösung als sehr interessante Möglichkeit bewährt, die sich immer weiter der Beugungslimitierung nähern [Thieme u. a., 1998].

Eine Fresnelsche Zonenplatte ist so konstruiert, dass die durch eine Absorptions- oder Phasenmaske transmittierte Röntgenstrahlung in einem Fokuspunkt konstruktiv interferiert. Diese Bedingung ist in Abbildung 1.5 illustriert und lässt sich durch den Satz 


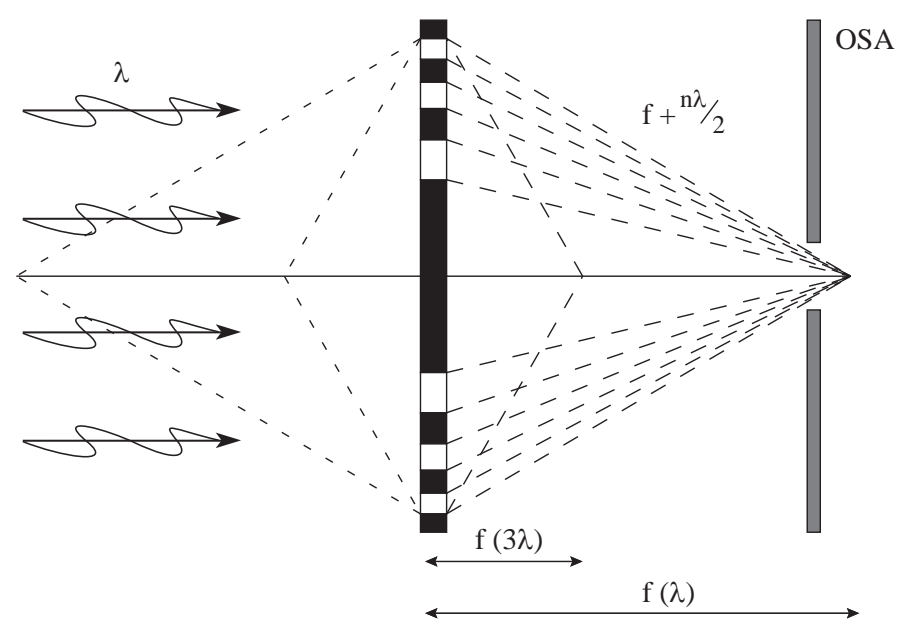

Abbildung 1.5:

Konstruktion einer Fresnelschen Zonenplatte. Die weißen Schichten seien transparent, während die schwarzen opak seien. Durch konstruktive Interferenz entsteht ein Fokus. Eine kürzere Wellenlänge bewirkt eine größere Brennweite. Es entstehen auch Brennpunkte höherer Beugungsordnungen und negative Ordnungen, die einen divergenten Strahl bewirken. Eine ordnungssortierende Apertur (OSA) kann die gewünschte fokussierende Ordnung selektieren und den Hintergrund minimieren.

des Pythagoras im Zonenplattenbildungsgesetz formulieren:

$$
f^{2}+r_{n}^{2}=\left(f+\frac{n \lambda}{2}\right)^{2}
$$

$f$ ist dabei die Brennweite, $r_{n}$ der Radius der $n$-ten Zone und $\lambda$ die Wellenlänge der Strahlung. Für $f \gg n \lambda / 2$ vereinfacht sich der Ausdruck zu

$$
r_{n}^{2} \approx \sqrt{n \lambda f}
$$

Von den Anforderungen und der Herstellungstechnik abhängig werden solche Geometrien zur Erzeugung eines Linien- oder Punktfokus produziert. Um die Effizienz einer solchen Zonenplatte zu erhöhen, kann die in Abbildung 1.5 opake Zone durch eine um $\lambda / 2$ phasenschiebende ersetzt werden.

Die Breite einer einzelnen Zone kann durch

$$
\begin{aligned}
d_{n} & =r_{n+1}-r_{n} \\
& =\sqrt{\lambda f(n+1)}-\sqrt{\lambda f n} \\
& \approx \sqrt{\frac{\lambda f}{4 n}}
\end{aligned}
$$

berechnet werden. Die kleinste Zonenbreite $a$, ein für die Herstellung wichtiger Para- 
meter, bestimmt die Zahl der strukturierbaren Zonen und damit auch die Apertur und das Auflösevermögen der Optik, das in der kleinsten Zonenbreite $\delta_{r}=1,22 a$ angegeben wird.

Die Effizienz $\eta_{m}$ einer abwechselnd absorbierenden und transparenten Zonenplatte in der jeweiligen Ordnung ist gegeben durch [Attwood, 1999]:

$$
\eta_{m}= \begin{cases}1 / 4 & \text { für } m=0 \\ 1 / m^{2} \pi^{2} & \text { für } m \text { ungerade } \\ 0 & \text { für } m \text { gerade. }\end{cases}
$$

Eine Steigerung der Effizienz der meist mit einer binären Maske hergestellten Zonenplatten kann durch eine Annäherung an die Kinoform der Fresnel-Linse erreicht werden, die jedoch noch höhere Anforderungen an den Herstellungsprozess stellt [Tamura u. a., 2009; Alianelli u. a., 2011].

Nicht alle Zonenplatten haben eine vollständige Apertur. So kann beispielsweise ein experimentell bedingter Mittenstopp die innere Apertur verdecken oder aufgrund von Limitierungen des Herstellungsprozesses werden nicht alle Zonen strukturiert. Solche Zonenplatten haben eine eingeschränkte Apertur und analog zu klassischen refraktiven Optiken nimmt das räumliche Auflösevermögen ab. Es ist dann gegeben durch die Wellenlänge $\lambda$ und die numerische Apertur $N A$ [Vinogradov und Mitrofanow, 2009]:

$$
\delta_{r}=\frac{\lambda}{N A} .
$$

Für weiche Röntgenstrahlung wurden stetig kleinere Zonenbreiten demonstriert, die heute bis zu $12 \mathrm{~nm}$ klein sind. Dazu siehe beispielsweise für weiche Röntgenstrahlung:

- $20 \mathrm{~nm}$ Auflösevermögen bei 2,07 nm Wellenlänge [Chao u. a., 2003],

- $15 \mathrm{~nm}$ Auflösevermögen bei 1,52 nm Wellenlänge [Chao u. a., 2005],

- $12 \mathrm{~nm}$ Auflösevermögen bei 1,75 nm Wellenlänge [Chao u. a., 2009a,b]

und für harte Röntgenstrahlung:

- $500 \mathrm{~nm}$ Auflösevermögen bei $100 \mathrm{keV}$ Photonenenergie [Kamijo u. a., 2003],

- $30 \mathrm{~nm}$ Auflösevermögen (Fokus 3. Ordnung) bei $8 \mathrm{keV}$ Photonenenergie [Yin u. a., 2006],

- $40 \mathrm{~nm}$ Aufösevermögen bei $8 \mathrm{keV}$ Photonenenergie [Chu u. a., 2008],

- $15 \mathrm{~nm}$ Aufösevermögen bei 6,2 keV Photonenenergie [Vila-Comamala u. a., 2010].

Bei der Herstellung kommen zumeist lithografische oder holografische Belichtungs- und Ätzverfahren zum Einsatz. 
Ein alternativer Ansatz ist die Deposition von transparenten sowie absorbierenden oder phasenschiebenden Schichten auf ein ebenes oder rundes Substrat. In die entsprechende Länge geschnitten lässt es höhere Aspektverhältnisse zu, wie sie für harte Röntgenstrahlung wegen der geringeren Wechselwirkung notwendig sind und dabei höhere Effizienzen zulassen. Diese Multischicht-Laue-Linsen erzeugen je nach Art des Substrats einen Linien- oder Punktfokus. Auch bei ihnen werden für harte Röntgenstrahlung immer kleinere Foki demonstriert. Dazu siehe beispielsweise:

- $30 \mathrm{~nm}$ Linienfokus bei 19,5 keV Photonenenergie [Kang u. a., 2006],

- $16 \mathrm{~nm}$ Linienfokus bei 19,5 keV Photonenenergie [Kang u. a., 2008],

- $28 \mathrm{~nm}$ Linienfokus bei $20 \mathrm{keV}$ Photonenenergie [Takenaka u. a., 2009],

- 13,1 nm Linienfokus bei $20 \mathrm{keV}$ Photonenenergie [Koyama u. a., 2011a],

- 300 - 400 nm Punktfokus bei 15,5 keV Photonenenergie [Tamura u. a., 2002],

- $280 \mathrm{~nm}$ Punktfokus bei $20 \mathrm{keV}$ Photonenenergie [Koyama u. a., 2011b].

Für weiche Röntgenstrahlung wurden vergleichbare Optiken noch nicht demonstriert und werden in Liese u. a. [2011] und Reese u. a. [2011] und dieser Arbeit vorgestellt. 


\section{Kapitel 2}

\section{Targetkonzepte und deren Anwendung}

\section{Einleitung}

Viele Experimente mit XUV-Strahlung sind auf brillante Quellen und einen hohen Photonenfluss angewiesen, wie man ihn vor allem an Elektronenspeicherringen findet. So bietet die Röntgenmikroskopie im Wellenlängenbereich des Wasserfensters $(2,2-4,4 \mathrm{~nm})$ die Möglichkeit, mikroskopische Bilder mit hoher räumlicher Auflösung von anorganischen, organischen und biologischen Probesystemen in wässriger Umgebung anzufertigen. Diese sich stark entwickelnde Technologie ist inzwischen an den meisten Synchrotronstrahlungsquellen der Welt vertreten und hat sich dabei als Standardmethode in vielen Anwendungsbereichen etabliert [Quitmann u. a., 2009]. Ihre Verfügbarkeit ist jedoch durch den aufwendigen Betrieb der Speicherringe begrenzt. Für den laborgestützten Einsatz der Röntgenmikroskopie im Wasserfenster, der einer größeren Zahl von Nutzern zugänglich ist, bedarf es daher geeigneter Laborstrahlungsquellen.

$\mathrm{Zu}$ diesem Zweck wurden verschiedene, relativ brillante Strahlungsquellsysteme mit hohem Photonenfluss für das Wasserfenster entwickelt, darunter Gasentladungsquellen mit einem

- Photonenfluss $\approx 4 \cdot 10^{13} \frac{\text { Photonen }}{\text { sr.Puls }}$ bei $2,88 \mathrm{~nm}$ Wellenlänge und $1175 \times 1175 \mu^{2}$ Quellfläche [Benk u. a., 2008].

Darüber hinaus Laserplasmaquellen mit verschiedenen Targetkonzepten:

- ein Ethanoltröpfchentarget mit $\approx 10^{12} \frac{\text { Photonen }}{\text { sr.Linienbreite.Puls }}$ bei $3,37 \mathrm{~nm}$ Wellenlänge und $25 \times 25 \mu^{2}$ Quellfläche (FWHM) [Berglund u. a., 2000],

- ein kryogenes Stickstoff-Flüssigstrahl-Target mit $\approx 10^{12} \frac{\text { Photonen }}{\text { sr.Linienbreite.Puls }}$ bei $2,48 \mathrm{~nm}$ Wellenlänge und $20 \times 20 \mu \mathrm{m}^{2}$ Quellfläche $(\mathrm{FWHM})$ [Jansson u. a., 2005; Takman u. a., 2007],

\footnotetext{
${ }^{2}$ Der von Firma Microliquids entwickelte und aufgebaute Kryostat wurde in gemeinsamer Arbeit mit Dr. Anton Kalinin und Dr. Christian Peth (Firma Microliquids) im Rahmen des Projektes „Labratory X-ray Microscope" in den Versuchsaufbau integriert und charakterisiert.
} 
- ein gasförmiges Stickstoff-Target mit $5 \cdot 10^{11} \frac{\text { Photonen }}{\text { sr.Puls }}$ bei $2,88 \mathrm{~nm}$ Wellenlänge [Peth, 2008],

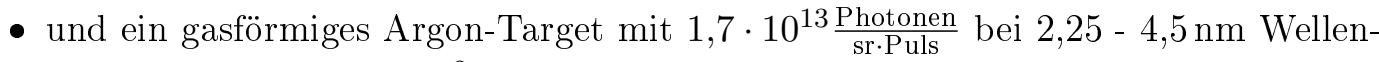
länge und $221 \times 105 \mu^{2}$ Quellfläche (FWHM) [Peth, 2008].

Auch von einer Wasserstrahlanode zur „klassischen“ Röntgenstrahlungserzeugung im Wasserfenster mit

- $\left(3 \cdot 10^{9} \frac{\text { Photonen }}{s \cdot \mu \mathrm{m}^{2} \cdot \mathrm{sr} \cdot \text { Linienbreite }}\right.$ bei $2,36 \mathrm{~nm}$ Wellenlänge und $15 \times 15 \mu \mathrm{m}$ Quellfläche (FWHM) [Skoglund u. a., 2010])

wurde demonstriert.

In diesem Kapitel werden zwei Targetkonzepte für Laserplasmaquellen für die Erzeugung von schmal- wie breitbandiger weicher Röntgenstrahlung im Labor diskutiert. Erstens eine Quelle auf Basis eines Gastargets (Kapitel 2.1) und zweitens eine auf Basis eines flüssigen kryogenen Argonstrahls, wobei die Entwicklung einer Quelle mit möglichst hoher Brillanz das Ziel ist. Ferner wird der Aufbau eines Wasserfenstermikroskops vorgestellt, in dem eine solche hochbrillante und breitbandige Laborquelle elementspezifische Kontrastmikroskopie an der Kalzium-Absorptionskante bei 3,56 nm Wellenlänge ermöglichen soll.

\subsection{Gastarget}

Zur Erzeugung eines Gastargets unter Vakuumbedingungen bedarf es eines gerichteten Gasstrahls, in dem Materialdichten erreicht werden, die zur hinreichenden Absorption des Laserstrahls führen und damit zum Zünden eines Plasmas ausreichen. Dieses wird durch eine Düse mit vereinfachter Lavalform erreicht, sodass der Gasstrom mit Überschallgeschwindigkeit gerichtet in die Kammer tritt und sich dort zunächst fortsetzt, bis er schließlich frei in das Vakuum expandiert. Durch Synchronisation von Laser- und Gaspuls wird ein Plasma gezündet und gleichzeitig der Gasballast in der Vakuumkammer auf ein Minimum reduziert.

Der Einsatz eines Gastarget ist - trotz seiner relativ geringen Teilchendichte und der daher geringen zu erwartenden Brillanz - im Betrieb mit Vorteilen behaftet, denn es handelt sich hierbei um ein sauberes Plasma, welches keine größeren Partikel oder schnelle Ionen in der Kammer freisetzt, die Beschädigungen an verschiedenen Teilen des Experiments - insbesondere den empfindlichen Optiken - verursachen können. Darüber hinaus kann durch die Wahl des Targetgases die Abstrahlcharakteristik des Laserplasmas einfach angepasst werden. Eine Vielzahl kompakter und schneller Ventile sind für diese und ähnliche Anwendung bereits entwickelt worden [Cross und Valentini, 1982; Proch und Trickl, 1989; Even u. a., 2000; Nugent-Glandorf u. a., 2002; Töttger, 2007]. 


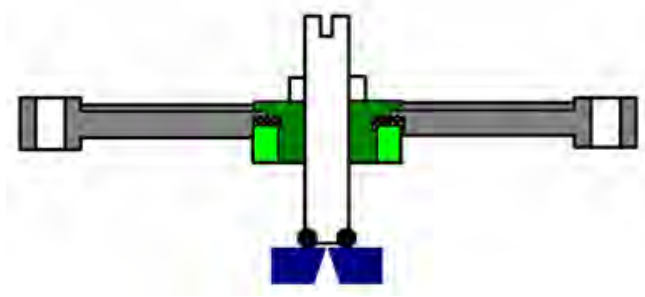

(a) Geschlossen

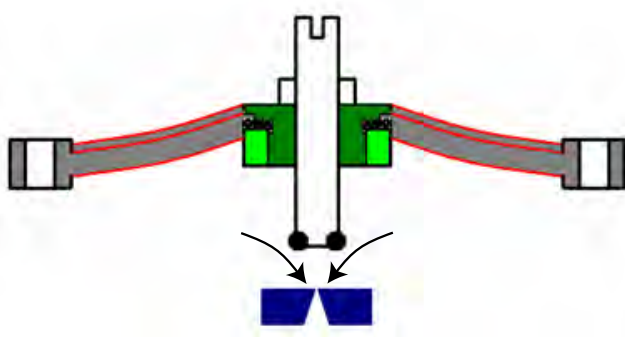

(b) Geöffnet

Abbildung 2.1:

Schematische Darstellung des Proch-Trickl-Ventils und dessen Schaltzustände. Unter Spannung (b) gesetzt verformt sich das System aus Piezo und Substrat und öffnet so das Ventil. Das umgebende Gas stömt durch eine Düse in vereinfachter Laval-Geometrie gerichtet in die Vakuumkammer [Großmann, 2009].

In vielen der durchgeführten Experimente kam ein Gastarget für das zu induzierende Plasma wegen seiner großen Vielseitigkeit und Stabilität zum Einsatz. Dessen Funktionsweise wird deshalb hier erläutert. Ein an die Bedingungen zur Erzeugung eines gepulsten Gasjets im Vakuum angepasstes Proch-Trickl-Ventil wurde optimiert [Großmann, 2009].

Ein Proch-Trickl-Ventil basiert auf einem Piezo-Disk-Translator, der unter Spannung verformt. Dadurch öffnet in Folge das Ventil wodurch das Einströmen des Targetgases in die Vakuumkammer ermöglicht wird. Die beiden Schaltzustände des schematisch dargestellten Ventils sind in Abbildung $2.1 \mathrm{zu}$ sehen. Wegen der kurzen Öffnungszeit des Ventils bei einer $\sin ^{2}$-förmigen Ansteuerspannung (maximal $750 \mathrm{~V}$ ) von etwa $800 \mu \mathrm{s}$ [Großmann, 2011] und dem gleichzeitig geringem Totvolumen liegen die Vorteile dieses Ventilsystems - gegenüber den zuvor verwendeten elektromagnetischen Ventilen - vor allem in seinem geringen Gasverbrauch und damit auch in der Verringerung des Gasballasts in der Vakuumkammer. Damit ist die mögliche Repetitionsfrequenz des Ventils höher.

Um einen in das Vakuum gerichteten Gasstrom zu erzeugen, wird das Gas über eine vereinfachte Laval-Düse in das Vakuum geleitet. In einer Entfernung von etwa $500 \mu \mathrm{m}$ wird durch den Laserfokus von $60 \mu \mathrm{m}$ Durchmesser ein heißes und dichtes Plasma in der Kammermitte gezündet. Für die Experimente im Wasserfenster werden dazu ein breitbandiger Emitter (Argon) und ein schmalbandiger Emitter (Stickstoff) gewählt.

Zur Charakterisierung der Form und Größe der erzeugten Plasmen wurden Lochkameraaufnahmen der Plasmen und Spektren der beiden Targetgase aufgenommen. Diese sind in Abbildung 2.2 dargestellt. Zum Zünden des Plasmas kam ein Innolas YMR800 (ausgewählte Parameter in Tabele 2.1) zum Einsatz. Das Spektrometer wurde durch Peth [2008] vorgestellt. Durch Filterung mit Titanfolie kann bei dem Stickstoffplasma eine einzelne Emissionslinie im Wasserfenster isoliert werden (Abbildung 2.2(d)). 
Tabelle 2.1:

Parameter der in den Laborexperimenten verwendeten Laser. Der Spitlight Hybrid wurde umgebaut um eine höhere Pulsleistung bei niedrigerer Repetitonsrate zu erzielen.

\begin{tabular}{|c|c|c|c|c|c|}
\hline & $\begin{array}{l}\text { Coherent } \\
\text { Infinity } \\
40-100\end{array}$ & $\begin{array}{l}\text { InnoLas } \\
\text { YMR800 }\end{array}$ & $\begin{array}{l}\text { InnoLas } \\
\text { Spitlight } \\
\text { Hybrid }\end{array}$ & $\begin{array}{l}\text { InnoLas } \\
\text { Spitlight } \\
600\end{array}$ & $\begin{array}{l}\text { Quantel } \\
\text { YG980 }\end{array}$ \\
\hline Wellenlänge & $\begin{array}{l}1064 \mathrm{~nm}, \\
532 \mathrm{~nm}\end{array}$ & $1064 \mathrm{~nm}$ & $1024 \mathrm{~nm}$ & $1024 \mathrm{~nm}$ & $\begin{array}{l}1024 \mathrm{~nm}, \\
532 \mathrm{~nm}, \\
355 \mathrm{~nm}\end{array}$ \\
\hline Pulsdauer & $3 \mathrm{~ns}$ & $7 \mathrm{~ns}$ & $9 \mathrm{~ns}$ & $6 \mathrm{~ns}$ & $7 \mathrm{~ns}$ \\
\hline Repetitionsrate & $1-100 \mathrm{~Hz}$ & $1 \mathrm{~Hz}$ & $\begin{array}{l}1-100 \mathrm{~Hz} \\
1-30 \mathrm{~Hz}\end{array}$ & $10 \mathrm{~Hz}$ & $1-10 \mathrm{~Hz}$ \\
\hline $\begin{array}{l}\text { maximale } \\
\text { Pulsenergie }\end{array}$ & $600 \mathrm{~mJ}$ & $900 \mathrm{~mJ}$ & $\begin{array}{l}300 \mathrm{~mJ} \\
590 \mathrm{~mJ}\end{array}$ & $700 \mathrm{~mJ}$ & $1600 \mathrm{~mJ}$ \\
\hline
\end{tabular}

Gasförmige Lasertargets haben jedoch im Wesentlichen zwei große Nachteile. Zum einen lassen sie sich wegen des hohen Gasballasts nicht bei einer hohen Repetitionsrate betreiben. Zum anderen hat das Targetmaterial nur eine geringe Dichte, wodurch die Absorption der Laserstrahlung weniger effektiv ist. Resultierend ist das induzierte Plasma weniger brillant und erzeugt einen geringeren mittleren Photonenfluss als es von den deutlich dichteren festen oder flüssigen Targets zu erwarten wäre.

Gegenüber dem Vorgängerventil [Peth, 2008] konnte die Photonenzahl pro Puls und Steradian mit dem Proch-Trickl-Ventil um eine Größenordnung auf $5 \cdot 10^{12} \frac{\text { Photonen }}{\mathrm{sr} P \text { Puls }}$ mit einem Stickstoff-Gastarget bei 2,88 nm Wellenlänge erhöht werden, die mögliche Repetitionsfrequenz erhöhte sich von $1 \mathrm{~Hz}$ auf $30 \mathrm{~Hz}$. 


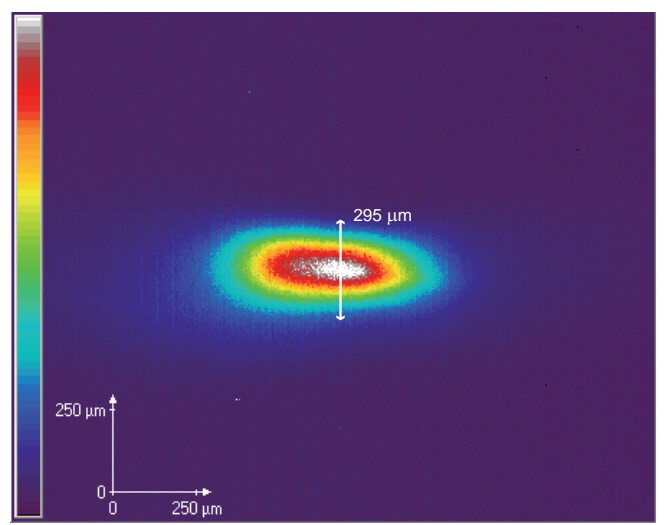

(a) Lochkameraaufnahme $(30 \mu \mathrm{m} \varnothing)$ eines Tigefilterten Stickstoffplasmas von $295 \times 823 \mu \mathrm{m}^{2}$ Größe $\left(1 / e^{2}\right)$ (Lochkamera mit Toshiba Teli CS8620, P43 Phosphorbeschichtung, $8 \mathrm{~ms} \mathrm{Be}$ lichtungszeit) [Reese u.a., 2011].

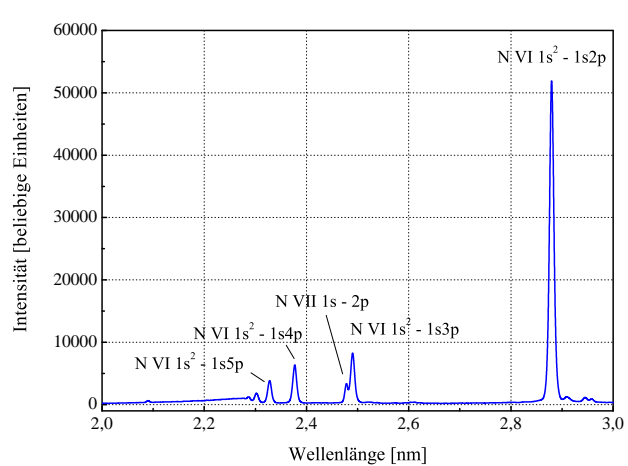

(c) Spektrum eines Stickstoffgastargets, einige der Übergänge sind detailliert angegeben [Großmann, 2011].

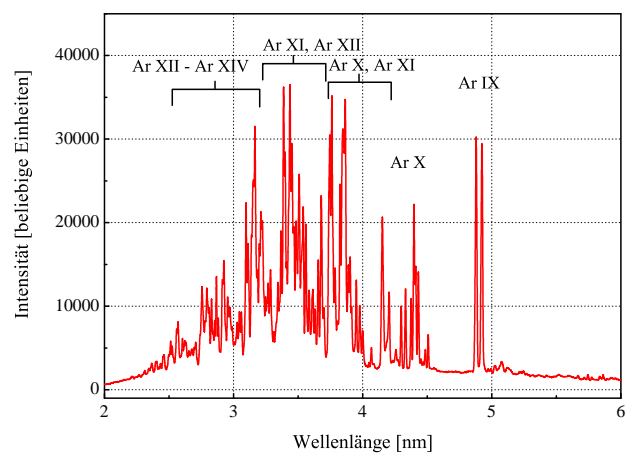

(b) Spektrum eines Argongastargets [Großmann, 2011].

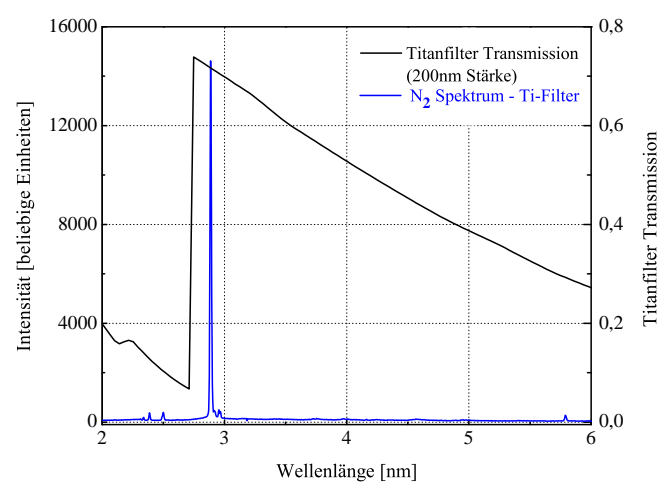

(d) Spektrum eines Stickstoffgastargets, gefiltert durch eine $200 \mathrm{~nm}$ Titanfolie. Die einzelne 2,88 nm Spektrallinie wird dabei freigestellt. [Reese u. a., 2011].

\section{Abbildung 2.2:}

Spektren der verwendeten Targetgase bei Verwendung eines Proch-Trickl-Ventils. Einige der Elementenübergänge sind zur Übersicht eingezeichnet. Es werden die lonisierungszustände nach Konvention in der Spektroskopie angegeben, in der beispielsweise ArI Argonatome und Ar IX achtfach ionisiertes Argon bezeichet. [Attwood, 1999]. 


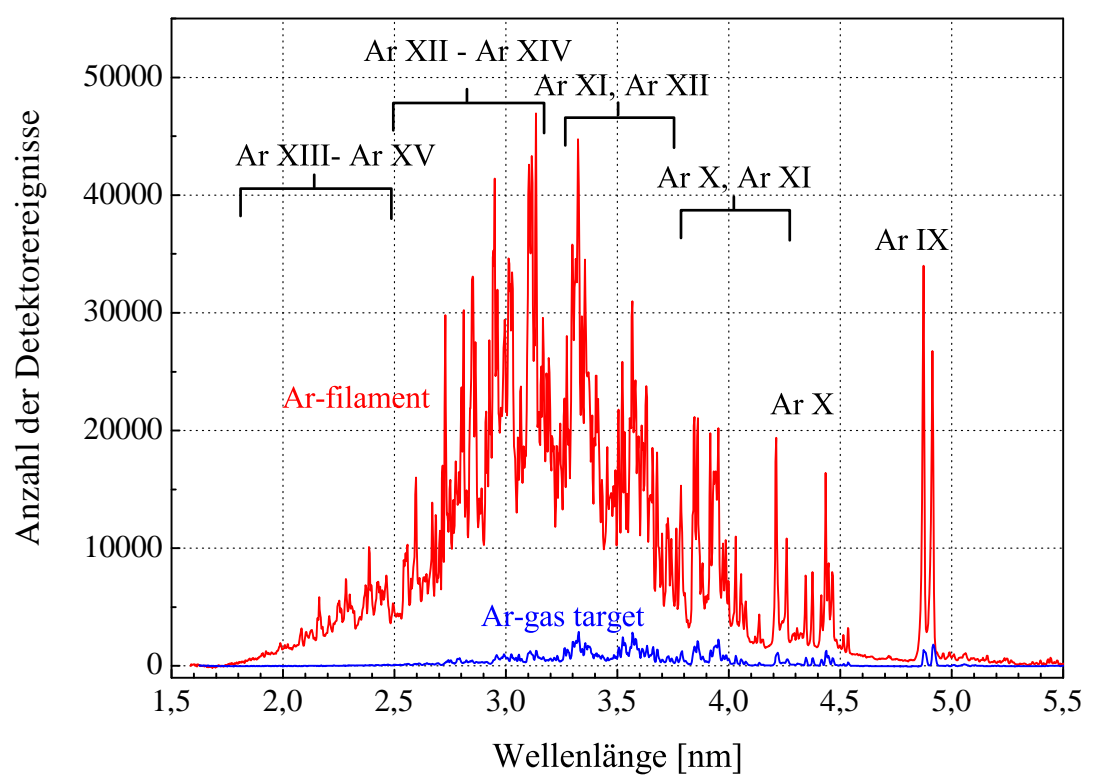

Abbildung 2.3:

Spektrum eines Gastarges verglichen mit einem kryogenen Festkörpertarget [Peth u. a., 2007].

\subsection{Flüssiges Argon als Targetmaterial}

Da gerade mikroskopischen Verfahren zur Erzeugung von Bildern mit hoher räumlicher Aufösung eine Quelle mit hoher Brillanz voraussetzen, werden massenlimitierter Festkörper- oder Flüssig-Targets eingesetzt um die Brillanz der Laserplasmaquelle entscheidend zu erhöhen. Mit solchen Laborquellen konnten mikroskopische Aufnahmen mit einer Auflösung bis unter $50 \mathrm{~nm}$ bei Belichtungszeiten von wenigen Minuten realisiert [Berglund u. a., 2000; Takman u. a., 2007; von Hofsten u. a., 2009] und auch kryotomographische Bilder angefertigt werden Bertilson u. a. [2011].

Abbildung 2.3 zeigt das Spektrum eines kryogenen massenlimitierten Laserplasmas. Man sieht die deutliche Steigerung der Intensität des Plasmas und die effektivere Ionisationsrate, die sich in höheren Photonenenergien ausdrückt. Die Brillanz dieser Quelle liegt beinahe beim Hundertfachen, verglichen mit der des Gastargets [Peth u. a., 2007; Peth, 2008].

Eine solch brillante und breitbandige Quelle soll in einem Experiment zur Spektromikroskopie im Wasserfenster verwendet werden. Dazu wurde ein kryogenes und flüssiges Argontarget entwickelt, dessen breitbandiges Spektrum erst später durch geeignete Optiken monochromatisiert werden soll. 


\subsubsection{Aufbau des Argonkryostaten}

Zur Erzeugung eines kryogenen und flüssigen Argonstrahls als Target für den Laser wurde ein Kryostat in Zusammenarbeit mit der Firma Microliquids entwickelt und optimiert. Der schematische Aufbau ist in Abbildung 2.4 dargestellt.

Die Zuleitung von Argon der Reinheit 4.8 findet über ein Stahlrohr statt. Das Gas wird zunächst in einem Aktivkohlefilter von möglichen Verunreinigungen befreit. In einem Vakuumbehälter, der über der Quellkammer montiert ist, befindet sich eine Kühlwendel in einem Bad aus flüssigem Stickstoff. Mechanisch wird der Behälter durch einen Ring von Teflonstäben stabilisiert. Das Argon wird in die Kühlwendel geleitet und kondensiert dabei. Zwei Partikelfilter von $15 \mu \mathrm{m}$ und $2 \mu \mathrm{m}$ Porengröße filtern das flüssige Argon von etwaigen Verunreinigungen. Danach tritt es in eine sich zur Düse verjüngende Glaskapillare (Fa. Microliquids, Austrittsöffnung etwa $30 \mu \mathrm{m} \varnothing$ ) und fließst daraufhin als Strahl von etwa $30 \mu \mathrm{m}$ Durchmesser durch die Vakuumkammer. Dort wird es von dem Skimmer aufgefangen. In diesem verdampft das Argon wieder und wird differentiell abgepumpt. Der gesamte Kryostat ist so auf zwei Platten gelagert, dass der Argonstrahl zum Laserfokus hin justiert werden kann. Auch der Skimmer kann entsprechend in xz-Ebene justiert werden.

Der Kryostat wird unter einem Druck von 2,3 bar betrieben um die Siedetemperatur des Stickstoffs zu erhöhen. Hierdurch wird eine stabile Arbeitstemperatur von $85 \mathrm{~K}$ zur Verflüssigung des Argons erreicht. Die Phasendiagramme der beiden Gase mit dem markierten Arbeitspunkt sind in Abbildung 2.5 gegeben.

\subsubsection{Betrieb des Argonkryostaten}

Abbildung 2.6 zeigt den Betrieb eines stabilen Argonstrahls. Der Abstand der Kapillare zum Skimmer wurde auf $45 \mathrm{~mm}$ festgelegt. Dieses sind $15 \mathrm{~mm}$ mehr als durch die abschattungsfreie XUV-Strahlführung bedingt ist. Jedoch wird so der Einfluss von Debris, Thermik und Schockwellen auf den empfindlichen Kapillarausgang minimiert.

Die Eigenschaften der Kapillare sind entscheidend für die Qualität und die Stabilität des erzeugten Argonstrahls. McCarthy und Molloy [1974] beschrieben fünf ausschlaggebende, geometrischen Parameter (siehe Abbildung 2.7), die das Strahlverhalten beeinflussen:

- Das Verjüngungsverhältnis $\beta=d / D$,

- das Düsenaspektverhältnis $A R=L / d$,

- der Kontraktionswinkel $\alpha$,

- die Stromlinienform der Düse und

- die Glattheit der Düseninnenseite. 
Diese Parameter sind experimentell nicht zugänglich, sodass keine verlässliche Vorhersage über die Qualität des Argonstrahls getroffen werden kann. Lediglich die Form der Düsenaustrittsöffnung, der Winkel zwischen der Düsenaustrittsebenennormalen zur Kapillarachse und die Unversehrtheit der Kapillaren- und Düsenwand können mit dem Lichtmikroskop inspiziert werden. Beispiele verschiedener Kapillare im Betrieb und unter dem Mikroskop sind in Abbildung 2.8 dargestellt.

Durch Verdampfen von Argon von der Strahloberfläche wird der Arbeitsdruck in der Kammer erhöht. Durch das differentielle Abpumpen des abdampfenden Argonstrahls und den Einsatz von zwei Turbomolekularpumpen wird ein Vakuum in der Kammer von weniger als $5 \cdot 10^{-3}$ mbar erreicht. Das ist ausreichend um über die gesamte Länge von $165 \mathrm{~cm}$ der Strahlführung im späteren Versuchsaufbau (Kapitel 2.3.1) eine Transmission von mehr als $90 \%$ der XUV-Strahlung zu gewährleisten (Abbildung 2.9). 


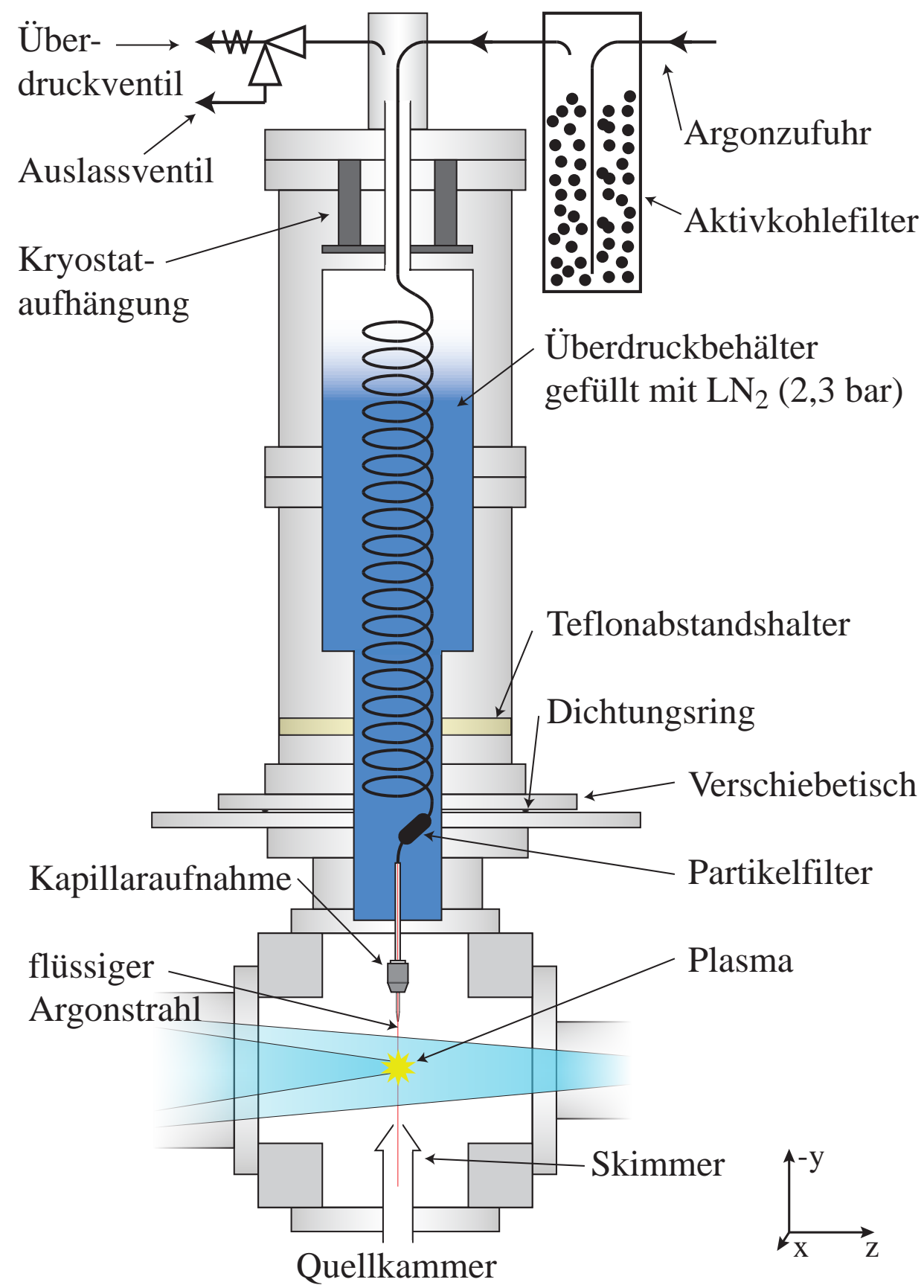

Abbildung 2.4:

Schematischer Aufbau des Argonkryostaten. Das im flüssigen Stickstoffbad verflüssigte Argon wird durch eine Kapillare in die Vakuumkammer geführt und als Target für das Laserplasma verwendet. Für die Integration im Gesamtaufbau siehe Abbildung 2.14 auf Seite 43 


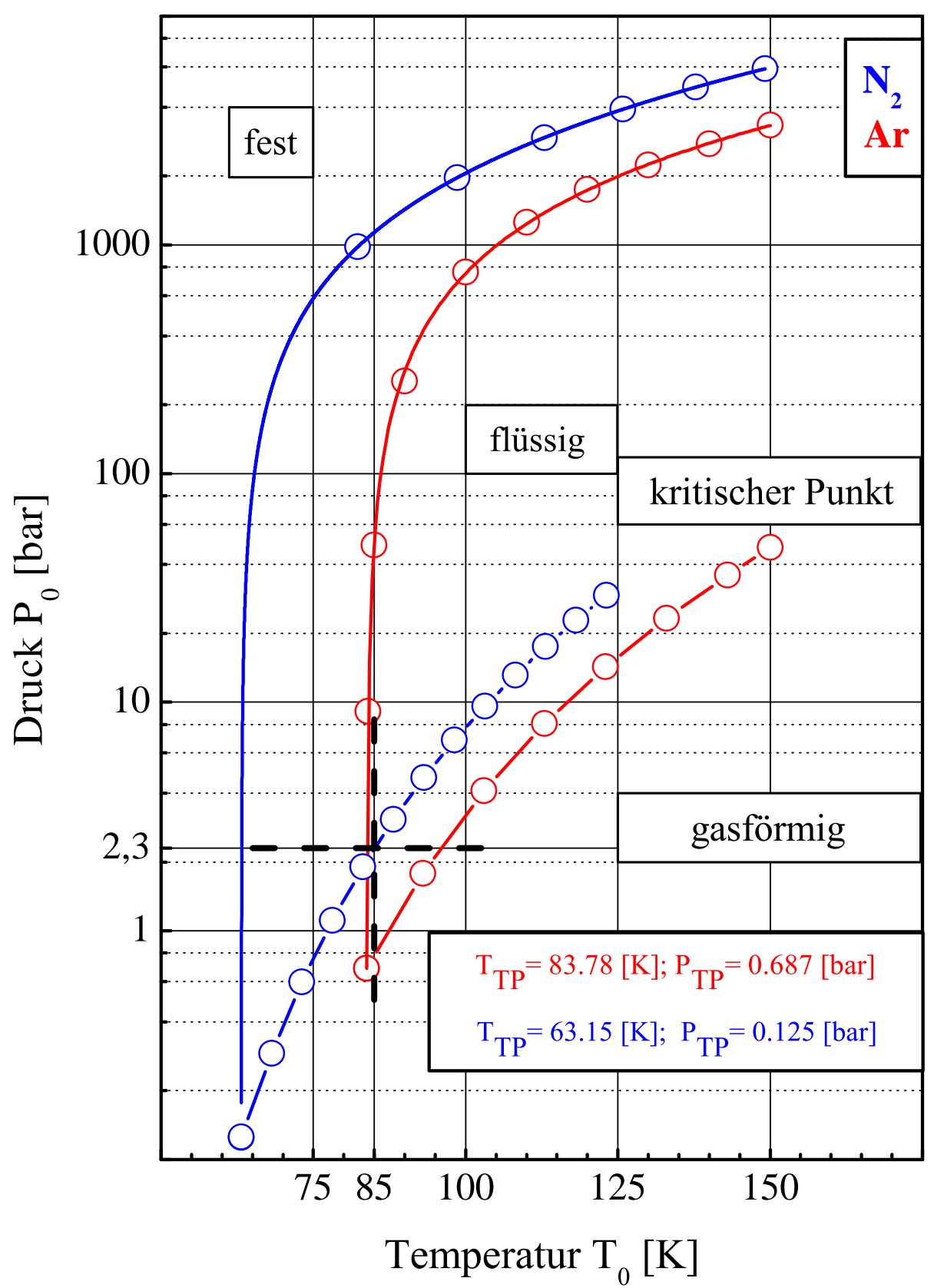

Abbildung 2.5:

Phasendiagramm von Stickstoff und Argon. Der Arbeitspunkt des Kryostaten ist durch ein Kreuz gekennzeichnet. 


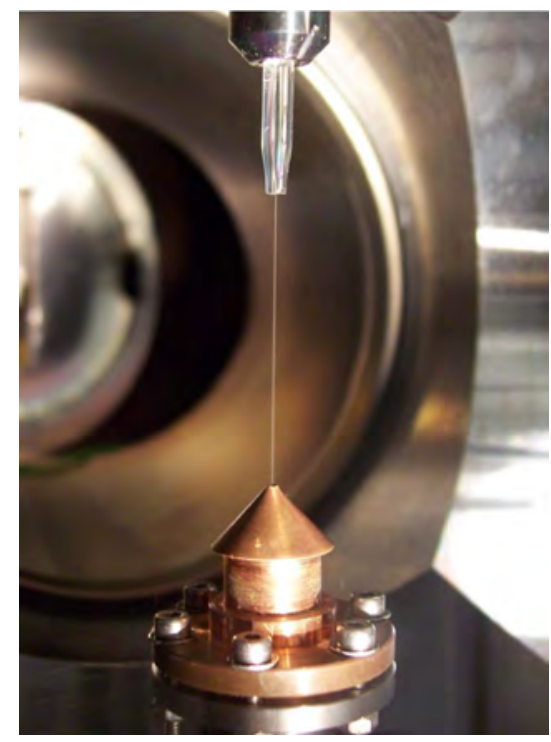

(a) Foto des gesamten Argonstrahls durch die Kammer.

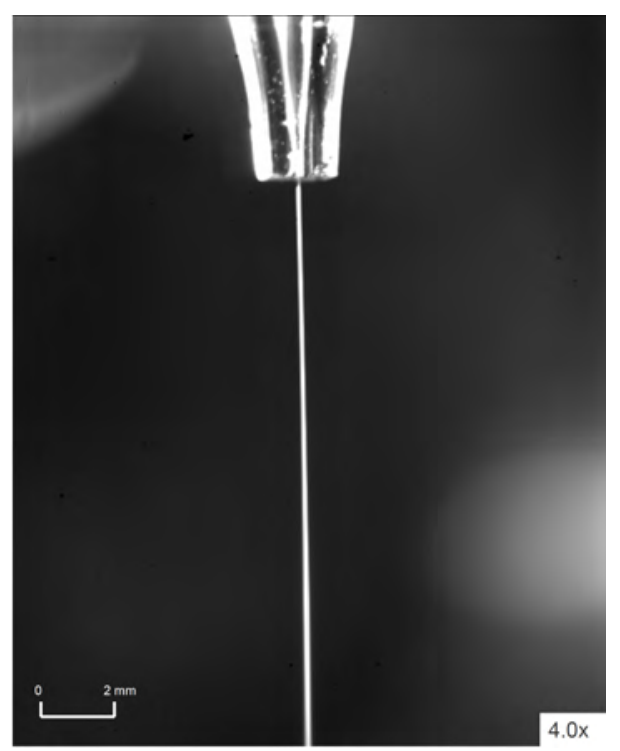

(b) Detailaufnahme des Strahlaustritts aus der Kapillare.

Abbildung 2.6: Fotos des kryogenen Argonstrahls in der Quellkammer bei verschiedenen Vergrößerungen.

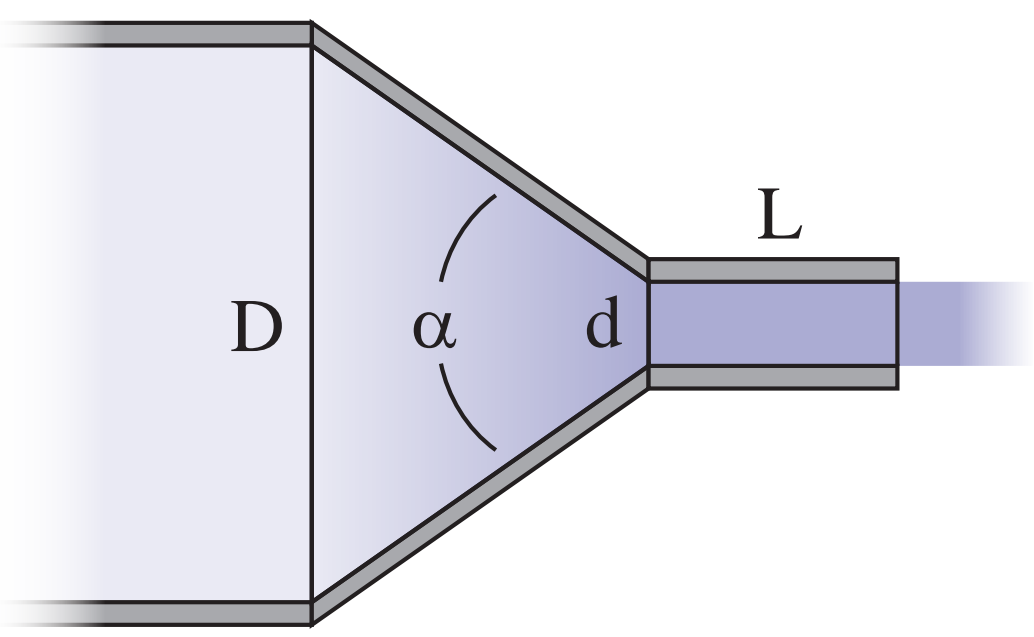

Abbildung 2.7: Geometrische Parameter einer beliebigen Düse [McCarthy und Molloy, 1974]. 


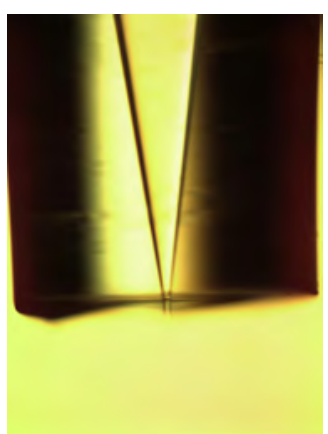

(a) Düsenaustrittsebenennormale liegt parallel zur Kapillarenachse.

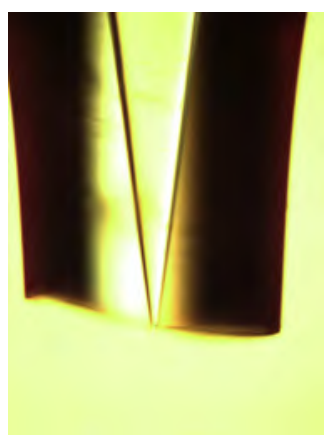

(d) Düsenaustrittsebenennormale abweichend zur

Kapillarenachse.

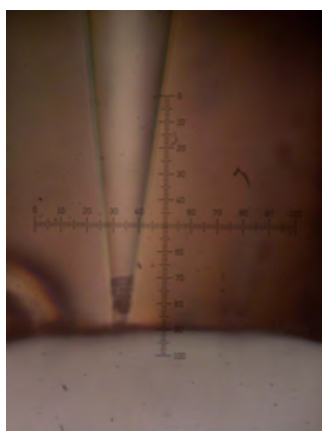

(g) Durch Partikel verdreckte und infolgedessen verstopfte Kapillare.

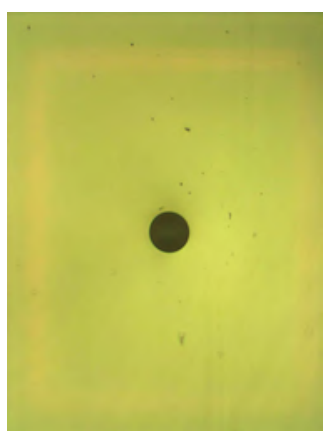

(b) Sauber und gerade gebrochene Düsenöffnung, etwa $30 \mu \mathrm{m} \varnothing$.

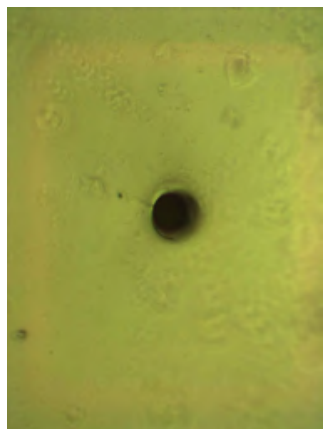

(e) Sauber, aber schräg gebrochene Düsenöffnung, etwa $30 \mu \mathrm{m}$ $\varnothing$.

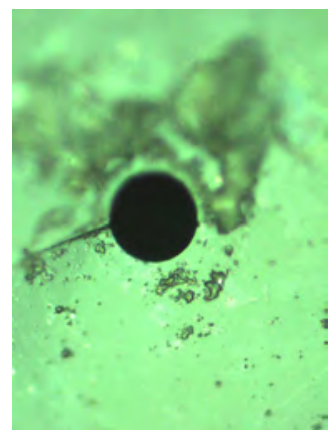

(h) Beschädigung des Düsenaustritts.

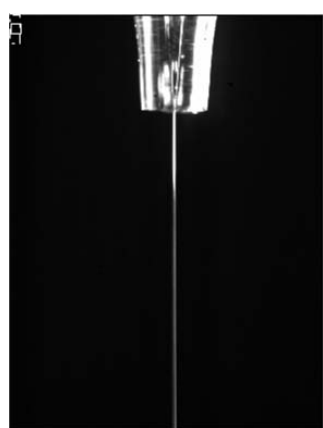

(c) Der resultierende Argonstrahl verhält sich wie gefordert.

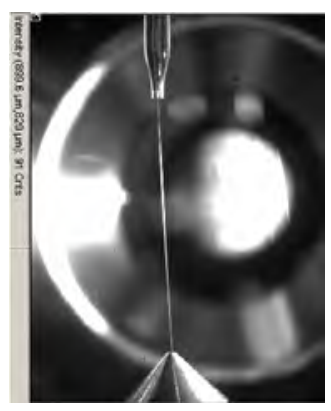

(f) Argonstrahl verläuft schräg durch Kammer.

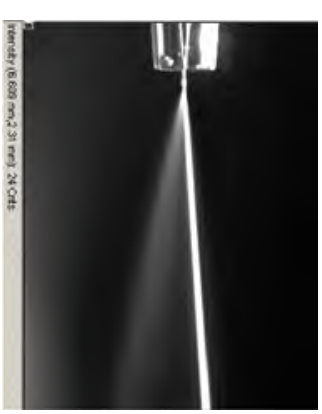

(i) Gestörter Argonstrahl.

Abbildung 2.8:

Betrieb des Argonkryostaten mit verschiedenen Kapillaren und deren lichtmikroskopische Eigenschaften. Zusätzlich sind mit dem Lichtmikroskop identifizierbare Störfaktoren dargestellt. 


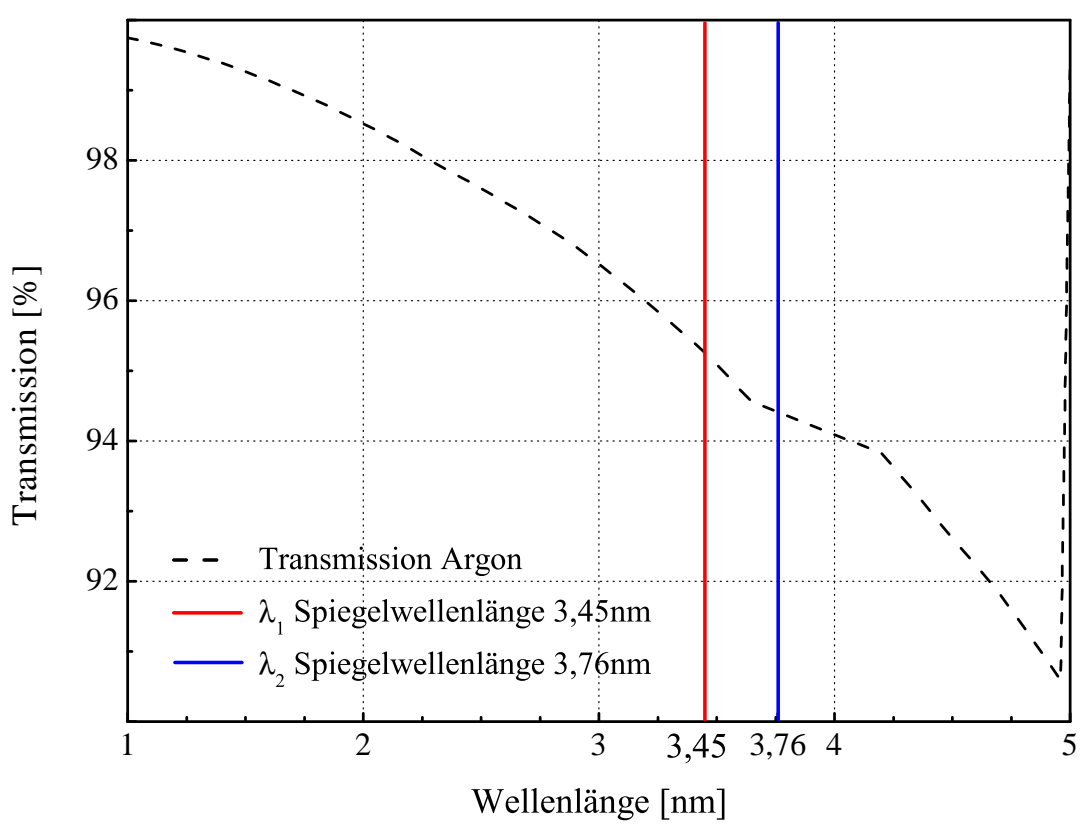

Abbildung 2.9:

Transmission von XUV-Strahlung (Daten aus Henke u. a. [1993]) im Versuchsaufbau unter einer angenommenen Argonatmosphäre, wie er in Kapitel 2.3.1 dargestellt ist. Die beiden durch den bichromatischen $\mathrm{Cr} / \mathrm{Sc}$-Spiegel reflektierten Wellenlängen sind gesondert gekennzeichnet. Beim erreichten Arbeitsdruck von $5 \cdot 10^{-3}$ mbar ist eine Transmission von mehr als $90 \%$ über die Strahlführung im Röntgenmikroskop von $165 \mathrm{~cm}$ gewährleistet. 


\subsubsection{Lagestabilität des Kryostaten und des Argonstrahls}

Die Eigenschaften des im Betrieb befindlichen Kryostaten, insbesondere die Lagestabilität des Argonstrahls, werden näher untersucht. Diese ist besonders wichtig, da der Überlapp von Laserfokus und Argonstrahl für die erfolgreiche Zündung eines Plasmas im Mikrometerbereich liegen muss. Dazu wurde ein Mikroskop mit sehr weitem Arbeitsabstand (Questar QM100, 15 - 35 cm Arbeitsabstand) durch ein Fenster in der Vakuumkammer auf den Strahl und die Kapillare gerichtet und jeweils deren Schwerpunkt mit einer Kamera (Toshiba Teli CS8620, $768 \times 494$ Pixel, 8,4 $\times 9,8 \mu^{2}{ }^{2}$ ) unter Einsatz der Analysesoftware MrBeam [Lübbecke und Schäfer] verfolgt. Die Ergebnisse dieser Messungen sind in Abbildung 2.10 aufgetragen.

Auffällig ist die starke Lageveränderung der Kapillare beim Abkühlen des Kryostaten, die in dessen thermischen Spannungen begründet sind. In y-Richtung sind diese besonders ausgeprägt und auch nach mehreren Stunden nicht in einem Gleichgewicht befindlich (Abbildung 2.10(b)). Allerdings ist die horizontale Lage der Kapillare während des Betriebs des Argonstrahls weitgehend konstant und verändert sich nur wenig (Abbildung 2.10(a) - Detail). Beobachtungen der Strahllage bei verschiedenen Abständen einer typischen Kapillare über jeweils einige Minuten zeigen, dass der Strahl normalerweise nicht permanent auf der gleichen Achse durch die Kammermitte liegt. Ferner wird der Strahl mit zunehmendem Abstand zur Düsenöffnung instabiler (Abbildung 2.10(c)). Bei einem Arbeitsabstand von $45 \mathrm{~mm}$ scheint die Lageinstabilität jedoch immer noch vertretbar. Sie macht dort etwa $40 \%$ des Strahldurchmessers aus. Bei Beobachtung der Lagestabilität über einen längeren Zeitraum zeigt sich jedoch, dass der Strahl sich fortlaufend um etwa den doppelten Strahldurchmesser verschieben kann (Abbildung 2.10(d)). Diese Dejustage durch thermische Effekte im Kryostaten während des Betriebs muss durch Nachjustage des Kryostaten korregiert werden.

All diese Untersuchungen wurden am Argonstrahl ohne Plasmazündung vorgenommen, da an der Oberfläche des Strahls gestreute und reflektierte Laserstrahlung die Kamera beschädigen würde. Somit kann während der Strahlungserzeugung keine Strahllagenüberwachung vorgenommen werden. Experimentell stellte sich heraus, dass bei höherer Repetitionsrate des plasmazündenden Lasers (Coherent Infinity 40 - 100, ausgewählte Parameter in Tabelle 2.1 auf Seite 26) die Argonstrahllage zunehmend instabil wurde, was häufig dazu führte, dass kein Plasma gezündet wurde. Eine mögliche Ursache hierfür sind Schockwellen, die bei der Plasmazündung durch den Strahl und die Kammer laufen und seine Lage destabilisieren. 


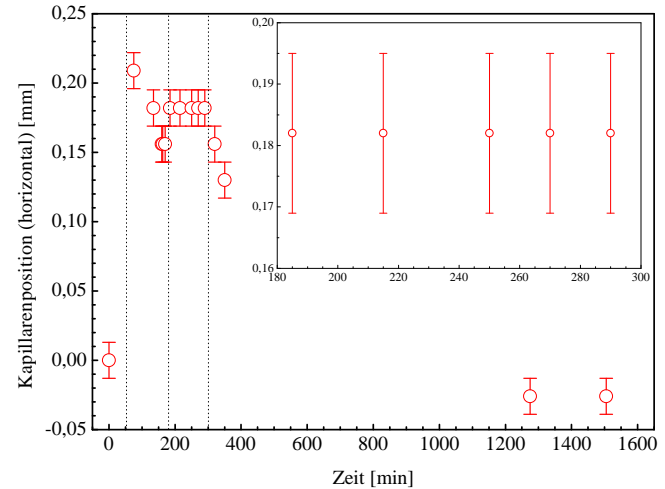

(a) Horizontale Lage (z-Achse) der Kapillare über die Abkühlungs-, Arbeits- und Aufwärmphase des Kryostaten, im Detail ist die Lage über den Betrieb des Strahls gezeigt.

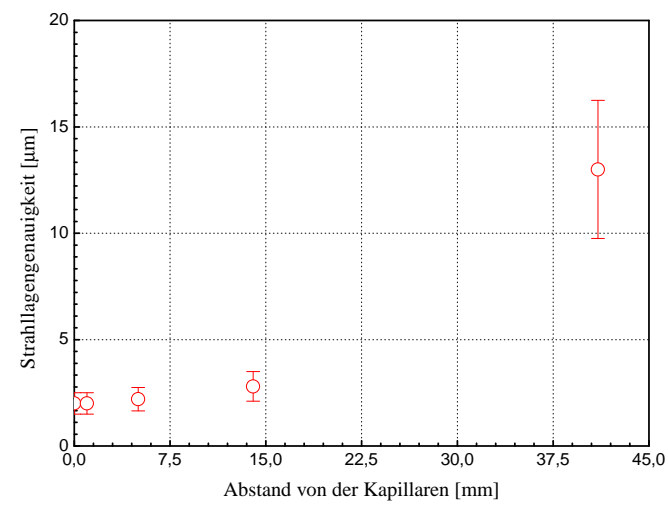

(c) Lagestabilität des Schwerpunktes des Argonstrahls in Abhängigkeit des Abstands von der Kapillare.

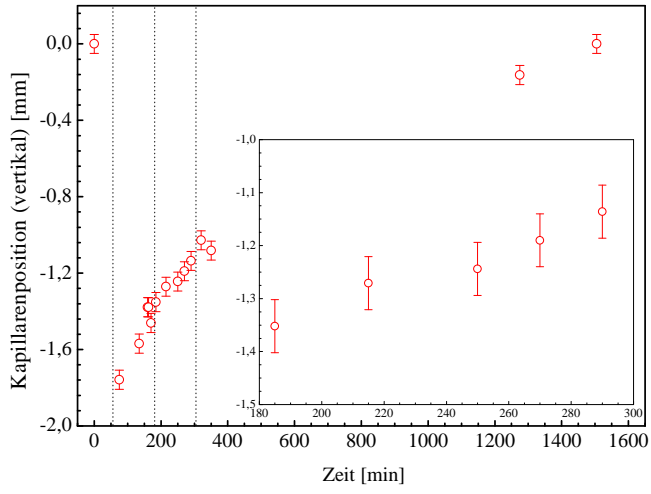

(b) Vertikale Lage (y-Achse) der Kapillare über die Abkühlungs-, Arbeits- und Aufwärmphase des Kryostaten, im Detail ist die Lage über den Betrieb des Strahls gezeigt.

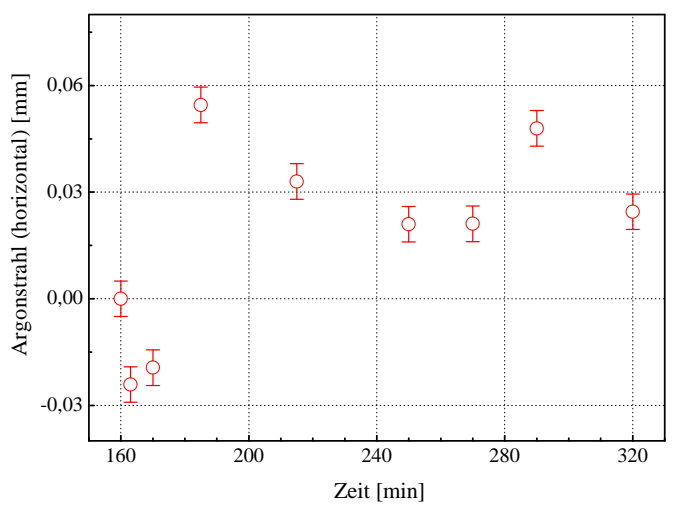

(d) Langzeitlagestabilität des Schwerpunktes des Argonstrahls.

Abbildung 2.10:

Lage der Kapillare und des Argonstrahls über einen Betriebszyklus des Kryostaten. Die Zeitmarken in Abbildung (a) und (b) zeigen: Bei 50 min ist der Druck im Stickstoffbehälter auf den Arbeitsdruck angestiegen, bei 150 Minuten ist die Temperatur im ganzen Kryostaten konstant, sodass der Argonstrahl eingeschaltet werden kann. Die Lage der Kapilare und des Strahls werden in getrennten Experimenten beobachtet. Bei 300 Minuten wird der Strahl abgeschaltet und die Kapillarenposition nach etwa neun Stunden wieder gemessen. 


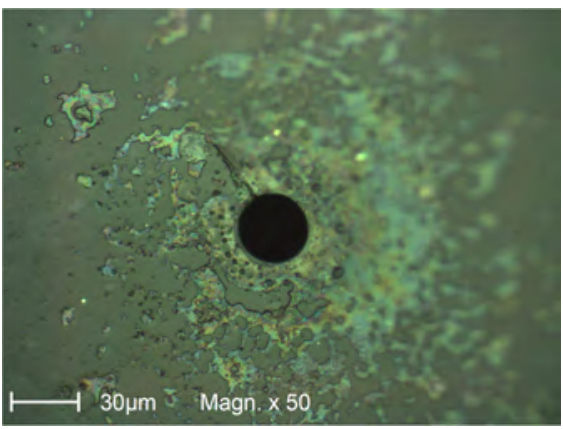

(a) Kapillarenöffnung vor DebrisExposition

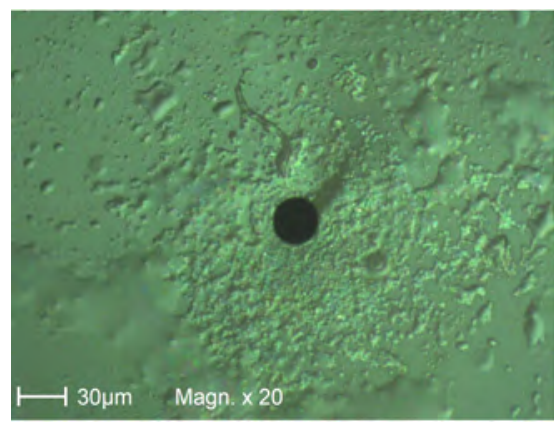

(b) Kapillarenöffnung nach DebrisExposition

Abbildung 2.11:

Beschädigung der Kapillare im Betrieb des Plasmas durch Debris. Durch Erosion der Oberfläche nahe des Düsenaustritts kann die Entstehung des freien Argonstrahls gestört werden.

\subsubsection{Debris-Erzeugung während des Betriebes}

Obwohl Edelgasatome nur schwer auf einer Oberfläche deponiert werden können, weil sie keine chemischen Bindungen eingehen und auf einer Oberfläche bei Raumtemperatur sofort in die Gasphase übergehen, kann Debris in Form von gefrorenen Argoneisfragmenten und schnellen Ionen durch Stoßprozesse empfindliche Beschichtungen und Optiken beschädigen [Kubiak u. a., 1995; Amano u. a., 2010]. Beide Arten von Debris wurden im Betrieb des Kryostaten beobachtet.

\section{lonisches Debris}

Bei Betrieb des Argonstrahls mit Zündung des Plasmas zeigt sich bereits nach wenigen Stunden, dass Erosionseffekte nahe des Düsenaustritts auf der Glaskapillarenoberfläche auftreten. Dieses kann dazu führen, dass der Austritt des flüssigen Argons aus der Düsenöffnung gestört wird und der resultierende Argonstrahl verstärkt Debris erzeugt (siehe Abbildung 2.8(i)) oder ganz zusammenbricht. Abbildung 2.11 zeigt die Oberfläche einer Kapillare nach einigen Stunden des Betriebs im Kryostaten, bei der bereits deutlich Erosionsschäden zu erkennen sind.

Um diese Erosionseffekte zu minimieren, muss der Abstand der Kapillare zum Plasma möglichst groß gewählt werden, damit durch vorhandenes Restgas die Argonionen weiter abgebremst werden. Die Standzeit der Kapillaren konnte so deutlich erhöht werden, obwohl dabei eine vergrößerte Lageinstabilität des flüssigen Argonstrahls in Kauf genommen wird. 


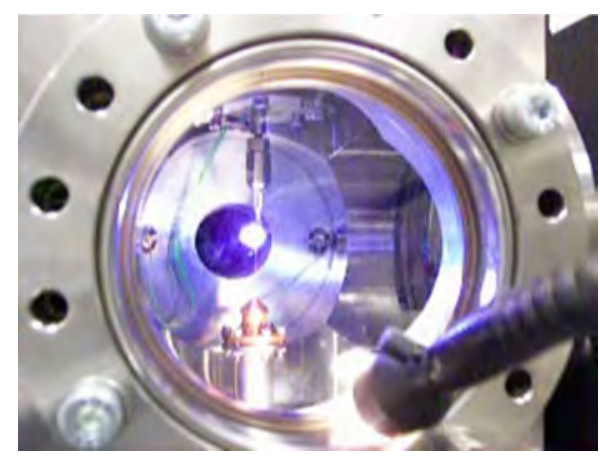

(a) Foto des Argonstrahls bei Zündung eines Plasmas.

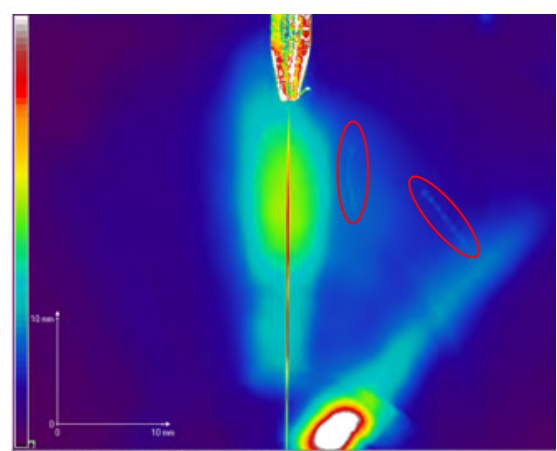

(b) Aufnahme des Argonstrahls durch ein Mikroskopobjektiv (Questar QM100, Toshiba Teli CS8620, 8 ms Belichtungszeit). Rot gekennzeichnet sind Debristeilchen, die auch bei ungestörtem Betrieb des Strahls entstehen können.

Abbildung 2.12: Foto des Betriebs des kryogenen Argonstrahls.

\section{Schneebälle}

Insbesondere beim Ein- und Ausschalten der Argongasversorgung entstehen, bevor es zu einem stabilen Argonstrahl kommt, Cluster gefrorenen Argons. Diese sogenannten Schneebälle schießen in die Anlage hinein und können durch ihre kinetische Energie Beschädigungen hervorrufen. Durch Blenden und Verschlüsse werden während dieser Phase die empfindlichen Bauteile des Experiments geschützt. Dennoch können jederzeit spontan durch Rayleigh-Oszillationen [J. W. Strutt, 3rd Baron Rayleigh, 1878] vereinzelt oder durch Störung des aus der Kapillaren austretenden Argons erhebliche Mengen an Schneebällen entstehen. Solche Störungen werden durch Verunreinigungen verursacht, die mit dem fließenden Argonstrom in die Kapillare gespült werden und diese am Düsenaustritt blockieren (siehe Abbildung 2.8(g)). Abbildung 2.12(a) zeigt ein Foto des im Betrieb befindlichen Argonstrahls bei Zündung eines Plasmas. Unter Gegenlicht können durch Beobachtung des Argonstrahls ohne Plasmazündung in der Kammer durch ein Mikroskop (Questar QM100) auch gestreutes Licht von Debris-Teilchen beobachtet werden (Abbildung 2.12(b)).

Um den beschädigenden Einfluss auf empfindliche Optiken zu untersuchen, wurden im Versuchsaufbau an Siliziumnitrid $\mathrm{Si}_{3} \mathrm{~N}_{4}$-Membranen von $100 \mathrm{~nm}$ Stärke montiert und der Kryostat willentlich zum Erzeugen von Schneebällen manipuliert. Auf solchen Membranen sind typischerweise diffraktive XUV-Optiken wie Zonenplatten aufgebracht, wie sie im späteren Röntgenmikroskop verwendet werden sollen. Es zeigt sich, dass bereits nach wenigen Minuten die Membran durch den Einschlag von Schneebällen vollständig zerstört werden kann (Abbildung 2.13). 


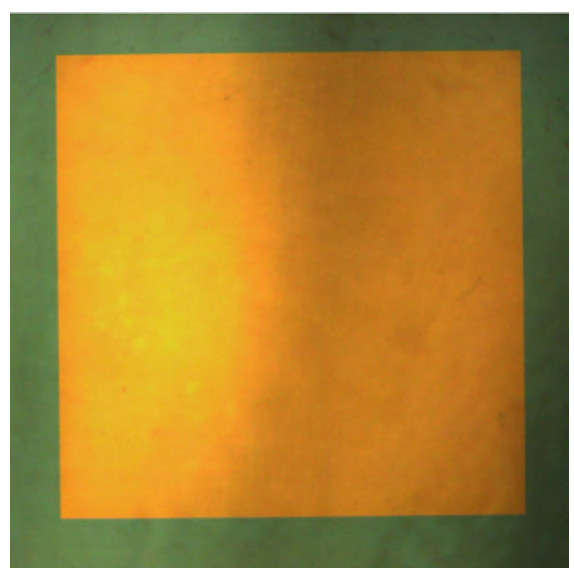

(a) Membran vor der Exposition.

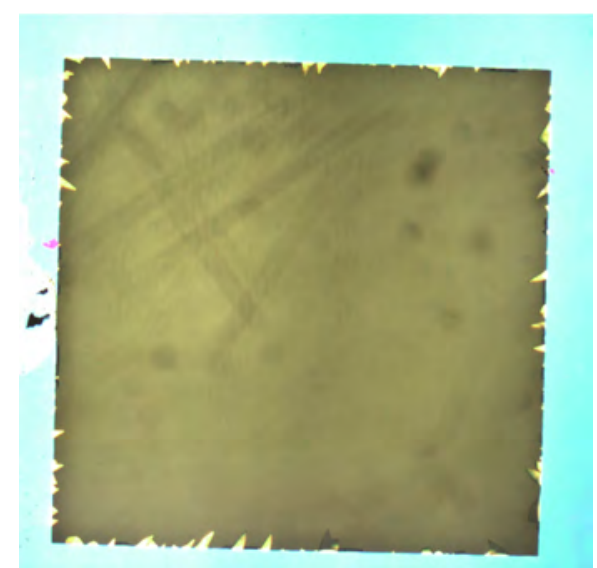

(b) Membran nach der Exposition.

Abbildung 2.13: Beschädigung von Siliziumnitrid-Membranen durch Schneebälle.

Durch Einführen eines Kreuzgitters mit $25 \mu \mathrm{m}$ Gitterkonstante $(15 \mu \mathrm{m}$ frei und $10 \mu \mathrm{m}$ Stegbreite) auf Höhe des Mittenstopps kann gewährleistet werden, dass große Argoncluster nicht mehr in den hinteren Versuchsaufbauteil gelangen können. Eine Beschädigung der Membranen wird nicht mehr beobachtet. Dennoch wird der Hauptteil der XUV-Strahlung transmittiert. Kleinere Schneebälle dringen jedoch hinter das Gitter und verursachen Beschädigungen an den Beschichtungen der CCD-Kameras. 


\subsection{Aufbau des Laborröntgenmikroskops}

Der kryogene Argonstrahl dient als Targetmaterial für eine hochbrillante Strahlungsquelle eines Laborröntgenmikroskops. Mit zwei genutzten Wellenlängen im Wasserfenster verfolgt das Experiment einen bichromatischen Ansatz, indem die elementspezifische Absorption des Kalziums ausnutzt wird. Im Folgenden wird der Aufbau des geplanten Gesamtexperiments geschildert.

\subsubsection{Design}

Der Aufbau des Gesamtsystems ist streng modular, sodass alle Aufbausegmente durch Vakuumverschlüsse voneinander getrennt sind. Eine schematische Zeichnung findet sich in Abbildung 2.14, ein Foto des Gesamtaufbaus in Abbildung 2.15.

\section{Laserplasmaquelle}

Breitbandige XUV-Strahlung wird in der Quellkammer (2) durch Zünden eines Plasmas durch den auf $30 \mu \mathrm{m}$ fokussierten Strahl eines Coherent Infinity 40-100 (ausgewählte Parameter in Tabelle 2.1 auf Seite 26) im massenlimitierten, kryogenen Argontarget erzeugt (siehe Kapitel 2.2). Abbildung 2.3 auf Seite 28 zeigt ein vergleichbares Spektrum eines kryogenen Argonfilamenttargets. Die so erzeugte Strahlung wird in alle Raumrichtungen emittiert.

\section{Kollektorspiegel}

Zum Monochromatisieren der einfallenden Strahlung, zum Nutzen eines möglichst großen Raumwinkels des von der Plasmaquelle emittierten Lichts und zum Abbilden der Plasmaquelle auf die Probe kommt ein sphärischer Vielschichtspiegel (Substrat: LLG e.V., Spiegelradius $550 \mathrm{~mm}, 75 \mathrm{~mm}$ optisch genutzter Durchmesser, 0,0824 quellseitige numerische Apertur, Vergrößerungsfaktor der Abbildung 1,73; Fraunhofer IOF: $\mathrm{Cr} / \mathrm{Sc}-$ Beschichtung) zum Einsatz, welcher über drei Vakuumdurchführungen justierbar in der Optikkammer (1) gehaltert ist. Der Spiegel hat eine für diesen Wellenlängenbereich hohe Reflektivität von $1 \%$ bei $3,435 \mathrm{~nm}$.

In einem später geplanten Ausbauschritt des Mikroskops wird er durch einen in vier Beschichtungs-Quadranten geteilten Vielschichtspiegel ersetzt (Fraunhofer IOF: $\mathrm{Cr}$ /Sc-Beschichtung). Jeweils die beiden gegenüberliegenden Quadranten sind für die Reflexion derselben Wellenlänge beschichtet. Durch eine Blende direkt vor dem Spiegel werden jeweils zwei gleichbeschichtete Quadranten zur Beleuchtung der Probe ausgewählt. Die Beschichtungen besitzen eine Reflektivität von etwa $4 \%$ bei $3,435 \mathrm{~nm}$ und $3,760 \mathrm{~nm}$. 


\section{Probenkammer}

Die Probe in der Probenkammer (3) wird durch den Kondensorspiegel mit einem vergrößerten Bild der Plasmaquelle am Ende des Beleuchtungsstrahlgangs beleuchtet. Diese befindet sich auf dem ersten von zwei mit Piezomotoren (3x Mechonics CU30 und 3x Mechonics CU30CL, $8 \mathrm{~mm}$ Hub, $50 \mathrm{~nm}$ Positioniergenauigkeit, CU30CL mit Längenmesssystem zur Positionskontrolle) in allen drei Raumrichtungen verfahrbaren Türmen. Der zweite dient der Aufnahme und Justage der abbildenden Zonenplatte. Die sich verändernde Brennweite der Zonenplatte (siehe Kapitel 1.3.2) bei Beleuchtung bei unterschiedlicher Wellenlänge wird durch Translation kompensiert.

\section{Zonenplatte}

Das Herzstück des Abbildungsstrahlengangs bildet die Zonenplatte (ZonePlates Ltd., $932 \mu \mathrm{m}$ Brennweite bei 3,435 nm Wellenlänge, $80 \mu \mathrm{m}$ Durchmesser, 500 Zonen, äußerste Zonenbreite $40 \mathrm{~nm}$, integrierter Mittenstopp der direktes Licht auf die CCD blockiert), deren größte numerische Apertur der des Spiegels bei 3,760 nm Wellenlänge angepasst ist. Die im Versuch gewählten Dimensionen ermöglichen eine Vergrößerung um den Faktor 500 bei einer räumlichen Auflösung von etwa $50 \mathrm{~nm}$. Die sich chromatisch verändernde Brennweiten der Zonenplatte (siehe Kapitel 1.3.2) wird durch Translation des Zonenplattenturms entlang der optischen Achse kompensiert. Da der Abstand zwischen Probe und Detektor konstant bleibt, wird die sich dadurch ergebende Veränderung des Vergrößerungsmaßstabs nachträglich numerisch korrigiert.

\section{Detektor}

Das vergrößerte Bild der Probe wird von einer rückseitig gedünnten CCD-Kamera (Roper Scientific PI-SX:1k, $1024^{2}$ Pixel, $13 \times 13 \mu \mathrm{m}^{2}$ Pixelgröße) detektiert. Ihr Abstand zur Probe bestimmt den Vergrößerungsmaßstab, der mit 500-fach geplant ist. Bei dieser Vergrößerung entspricht ein Pixel $26 \mathrm{~nm}$ in der Probenebene. Ein Auflösungsvermögen von $50 \mathrm{~nm}$ ist angestrebt. 


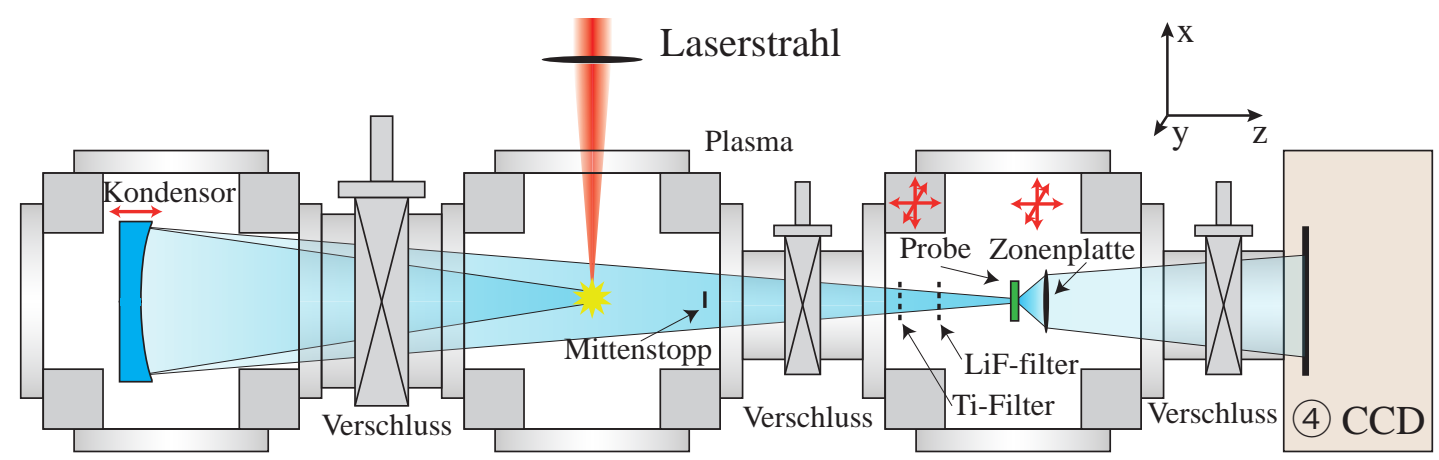

(1) Optikkammer

(2) Quellkammer

(3) Versuchskammer

Abbildung 2.14:

Schematische Zeichnung des Designs des Laborröntgenmikroskops. Der Aufbau ist modular entworfen, sodass die einzelnen Versuchsteile durch Verschlüsse getrennt werden können. In der Optikkammer (1) befindet sich der sphärische Kondensorspiegel, in der Quellkammer (2) wird das XUV-Strahlung erzeugende Plasma im kryogenen Argonstrahl gezündet (vergleiche Abbildung 2.4 auf Seite 31). In der Versuchskammer (3) befinden sich auf zwei motorisierten Türmen zum einen die Probe und zum anderen die Zonenplatte, die die Probe auf die CCD-Kamera (4) abbildet.

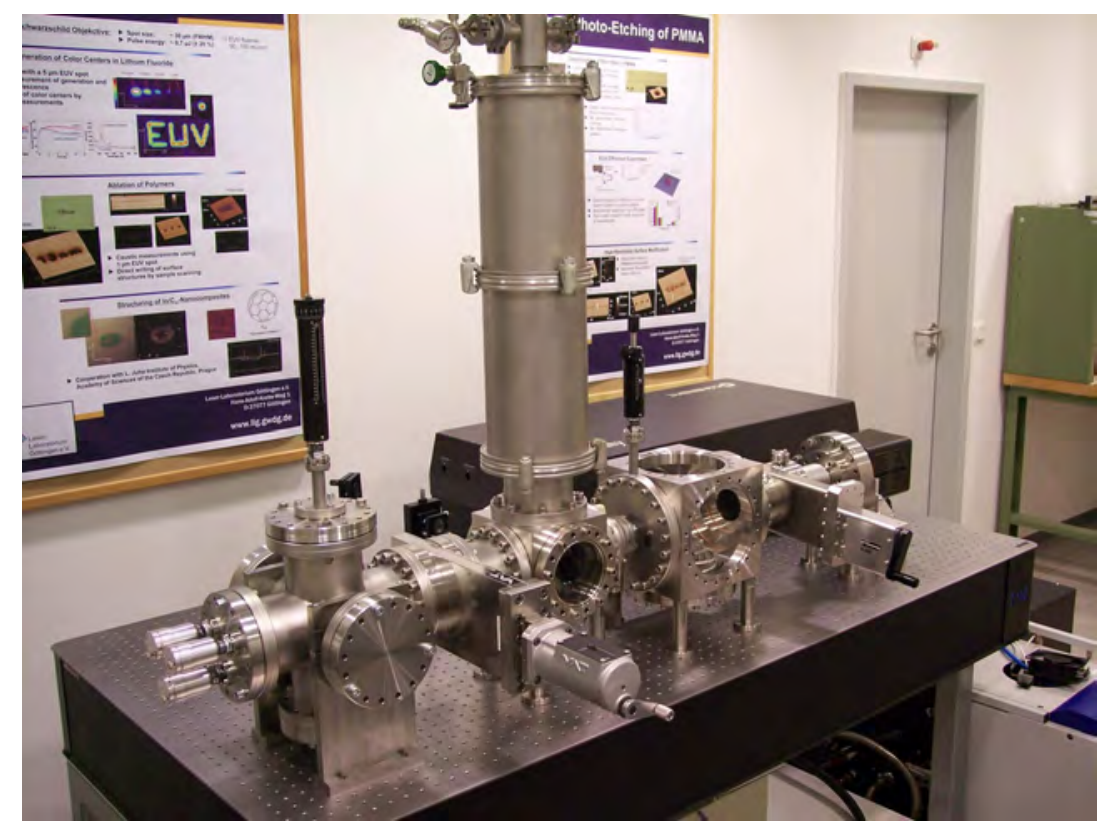

Abbildung 2.15:

Foto des Aufbaus zur Laborröntgenmikroskopie. Die einzelnen Module sind wie in Abbildung 2.14 dargestellt angeordnet. 


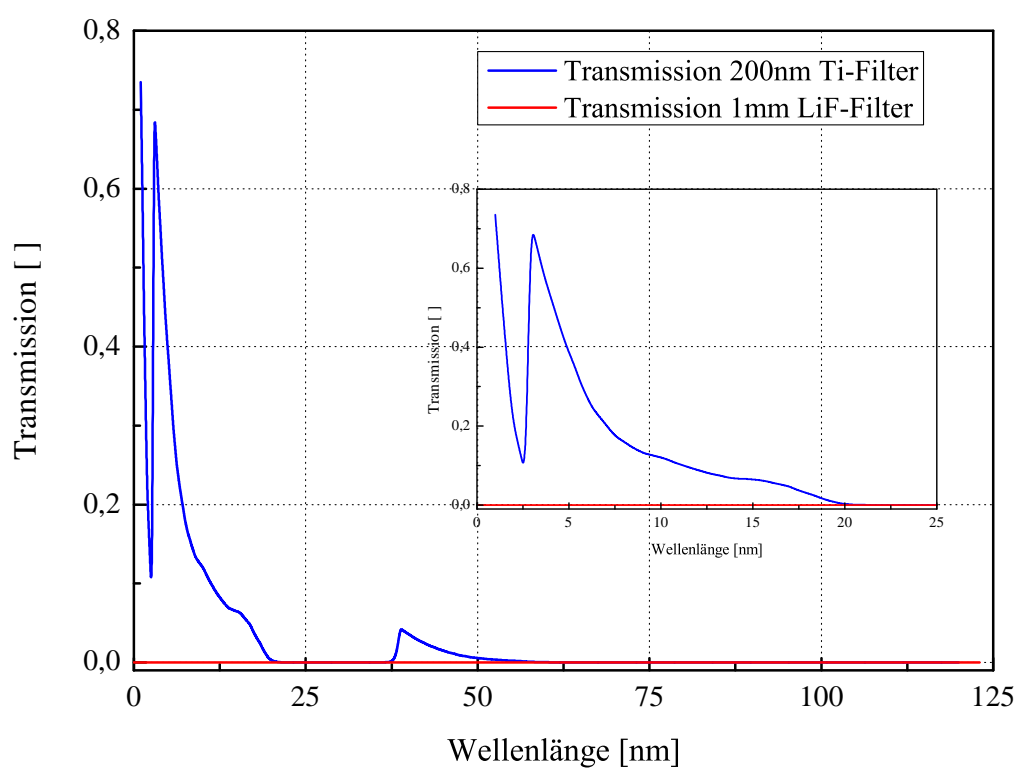

Abbildung 2.16:

Auftragung der Transmission der verwendeten Ti- und LiF-Filter im extrem ultravioletten und weichen Röntgenspektrum (Daten aus Henke u.a. [1993]).

\subsubsection{Erste Detektion von XUV-Strahlung}

Nach erfolgter Inbetriebnahme des Kryostaten und des Laserplasmas wird zunächst die Art der erzeugten Strahlung eindeutig identifiziert. Hierzu dienen zwei Titanfilter (Titanfolie auf einem Stützgitter) von je $200 \mathrm{~nm}$ Dicke (Ti-Folie: Jochen Herbst, Institut für Röntgenphysik, Universität Göttingen), der für Wellenlängen oberhalb von etwa $10 \mathrm{~nm}$ undurchlässig ist, und ein Lithium-Fluorid-Filter (Kristall von $1 \mathrm{~mm}$ Dicke), der für Wellenlängen im tiefen ultravioletten opak, im Optischen und nahen Infrarot jedoch transparent ist. Gemeinsam genutzt erlauben sie weder die Transmission von Röntgenstrahlung noch von sichtbarem und infrarotem Licht. Für den ultravioletten und weichen Röntgenbereich ist dies in Abbildung 2.16 dargestellt. Zur Begrenzung der Beleuchtungsfläche wurde eine Lochblende von $2 \mathrm{~mm}$ Durchmesser in die Probenebene gebracht und durch diese sowohl das direkte, als auch das vom Kondensorspiegel reflektierte Licht durch eine CCD-Kamera (Toshiba Teli CS8620, $768 \times 494$ Pixel, 8,4 × 9,8 $\mu^{2}$ ) mit Quantenkonverter (P43 Phosphorbeschichtung) direkt hinter der Lochblende detektiert.

XUV-Strahlung kann das Titanfilter passieren, muss aber durch den dann eingefahrenen Lithium-Fluorid-Filter absorbiert werden. So kann ausgeschlossen werden, dass es sich bei der detektierten Strahlung um Streustrahlung oder andere Störungen handelt. In ersten Experimenten zeigten sich transmittiertes Licht durch beide Filter sowie Beugungserscheinungen, die am Stützgitter des Titanfilters auftraten. Diese können 


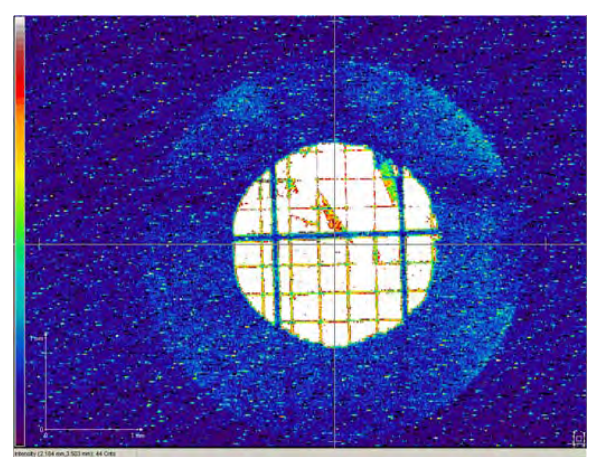

(a) Bild einer einzelnen Plasmazündung mit Ti-Filter.

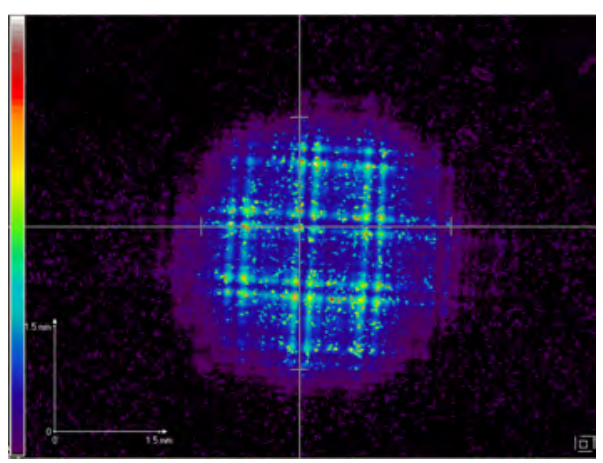

(c) Wie (b), mit Ti- und LiF-Filter.

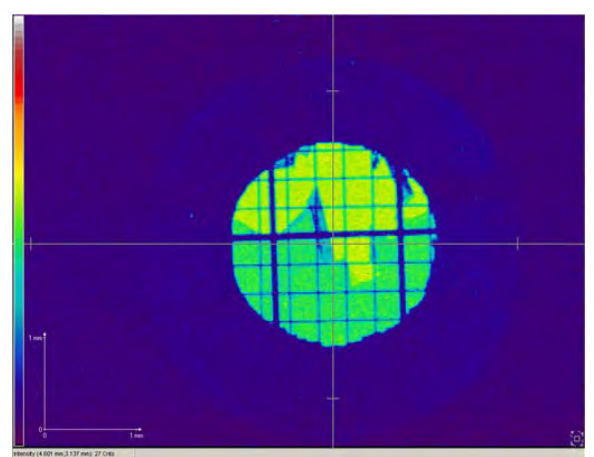

(b) Wie (a), jedoch über 25 Pulse gemittelt.

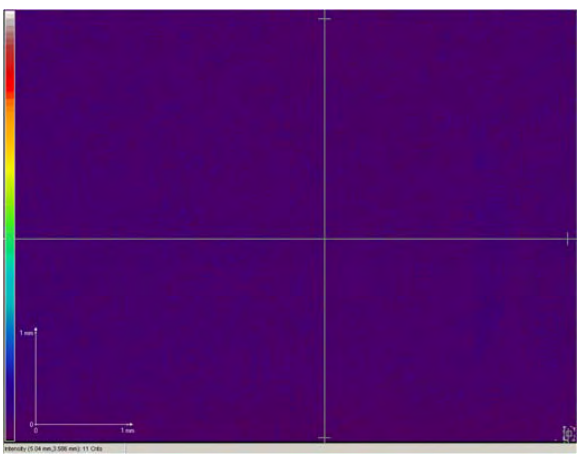

(d) Wie (b), mit Ti- und LiF-Filter und Umrüstung des Lasers auf $532 \mathrm{~nm}$ Wellenlänge.

\section{Abbildung 2.17:}

Detektion von XUV Strahlung (3,45 nm Wellenlänge) durch den Beleuchtungsstrahlgang (Toshiba Teli CS8620 mit P43 Phosphorbeschichtung). Durch Titan- und Lithium-Fluorid-Filters lassen sich komplementäre Spektralbereiche filtern, sodass sich die detektierte Strahlung als XUV-Strahlung identifizieren lässt (siehe Text).

als Laserstreulicht, reflektiert am Argonstrahl und aufgrund seiner hohen Intensität in geringem Anteil durch das Titanfilter transmittiert, identifiziert werden. Durch Umrüstung des Coherent Infinity 40 - 100 mit einem Frequenzverdoppler konnte dieses Licht durch die geringere Reflexion von Licht an Argon bei $532 \mathrm{~nm}$ unterdrückt werden. Abbildung 2.17 zeigt die erfolgreiche erste Detektion von XUV-Strahlung. 


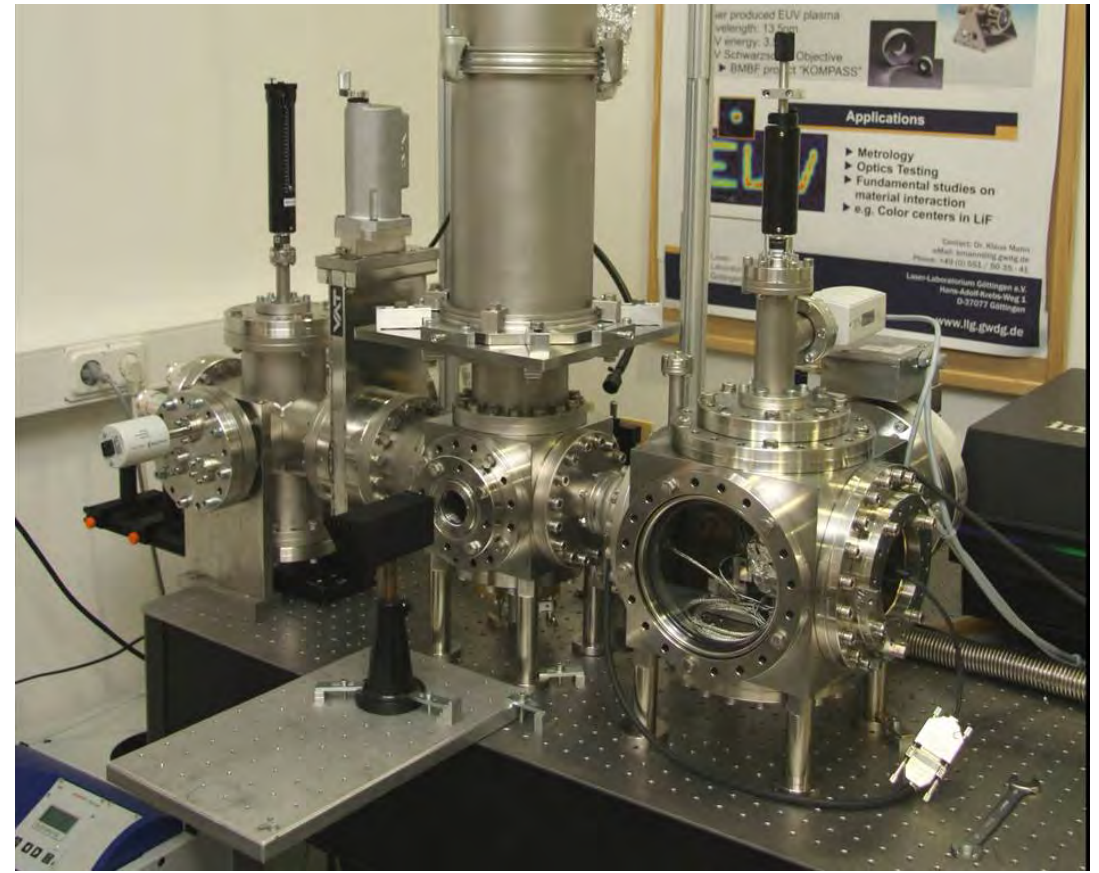

Abbildung 2.18:

Foto des Aufbaus zur Laborröntgenmikroskopie während der Justage des Beleuchtungsstrahlengangs.

\subsubsection{Justage des Beleuchtungsstrahlgangs}

Zur Beleuchtung der Probe muss zunächst der Beleuchtungsstrahlgang justiert werden. Dazu wird mit Hilfe eines Justagelasers eine optische Achse in der Kammermitte aufgespannt. Zum Festlegen des Ortes des Quellplasmas wird ein Luftplasma in der Kammer gezündet, das exakt auf der optischen Achse liegt. Mit Hilfe der Justageschrauben wird der Kondensorspiegel durch Abmessen des Abstands vom Luftdurchbruch positioniert und so justiert, dass sowohl das Licht des Justagelasers direkt zurückreflektiert wird, als auch das gesammelte, sichtbare Licht des Luftdurchbruchs auf der optischen Achse liegt. Anschließend wird der Aufbau abgepumpt und der Argonstrahl über den Laserfokus gefahren. Das nun auf der optischen Achse gezündete Plasma emittiert sowohl sichtbares Licht als auch XUV-Strahlung. Mit Hilfe des sichtbaren Lichts kann eine Vorjustage durchgeführt werden. Das Einfahren eines Titanfilters von $200 \mathrm{~nm}$ Dicke in den Strahlengang filtert alle Strahlung des Plasmas, die nicht im XUV-Bereich liegt. An der Stelle der Probe wurde anschließend eine CCD-Kamera (Toshiba Teli CS8620) mit Quantenkonverter (P43 Phosphorbeschichtung) gebracht, um den Beleutungsfleck zu detektieren. Ein Foto des Aufbaus während der Justage des Beleuchtungsstrahlengangs ist in Abbildung 2.18 gegeben.

Während der Justage des Beleuchtungsstrahlgangs wurde deutlich, dass zum einen 


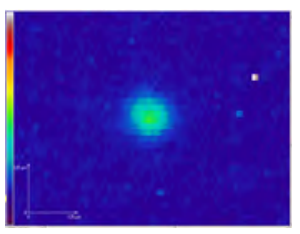

(a) $-500 \mu \mathrm{m}$

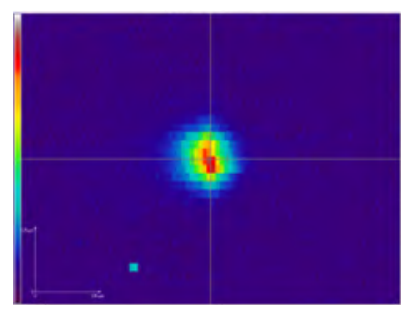

(b) optimale Justageposition

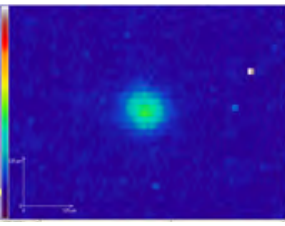

(c) $+500 \mu \mathrm{m}$

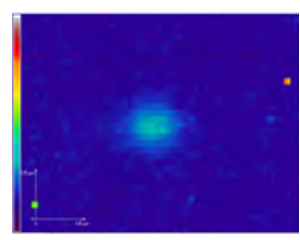

(d) $+1000 \mu \mathrm{m}$

Abbildung 2.19:

Justage des Kondensorspiegels. Die Plasmaquelle und der Detektor stehen fest, während der Kondensorspiegel verfahren wird. Bei einer guten Vorjustage mit sichtbarem Licht lässt sich die optimale Position des Kondensorspiegels mit minimaler Bewegung des Spiegels auffinden. Der in (b) dargestellte Beleuchtungsfleck $\left(3,45 \mathrm{~nm}\right.$ Wellenlänge, Ti-gefiltert) misst $60 \times 95 \mu \mathrm{m}^{2}$ (FWHM).

die Lagestabilität des kryogenen Argontargets bei Zünden eines Plasmas nicht stabil genug ist, da der Beleuchtungsfleck um mehrere $10 \mu \mathrm{m}$ auf der $\mathrm{x}$-Achse springt. Zum anderen ist das Debris-Problem noch nicht abschließend gelöst, da nach einiger Zeit der Justage die Phosphorbeschichtung der CCD-Kamera vollständig erodiert. Dennoch konnten Bilder vom Beleuchtungsfleck an der optimalen Spiegelposition 2.19(b) einer Größe von $60 \times 95 \mu^{2}$ aufgenommen werden (Designparameter $50 \times 50 \mu \mathrm{m}^{2}$ ).

\subsubsection{Photonendichte}

Die Photonenzahl pro Mikrometerquadrat und Sekunde ist eine entscheidende Kenngröße für die Röntgenmikroskopie, denn man braucht etwa $10^{8} \frac{\text { Photonen }}{\mu m^{2}}$ für eine erfolgreiche Belichtung [Guttmann u.a., 2003; Benk u.a., 2008]. Zur Bestimmung der durch das Plasma generierten und vom Kondensorspiegel eingesammelten Photonenzahl pro Puls wurde eine Photodiode (International Radiation Detectors Inc., AXUV-100) mit einer Quanteneffizienz von etwa $100 \frac{\text { Elektronen }}{\text { Photon }}$ bei $3,435 \mathrm{~nm}$ Strahlungswellenlänge anstelle der CCD-Kamera (vergleiche Kapitel 2.3.3) in den Versuchsaufbau gebracht. Deren Signal wurde in Abhängigkeit der Laserpulsenergie gemessen. Dabei wurde erstens das direkte Licht durch eine $650 \mu \mathrm{m}$ Lochblende und zweitens (nach Installation des Mittenstopps) die schmalbandige von Kondensorspiegel reflektierte XUV-Strahlung gemessen, die der späteren Beleuchtung der Probe dienen soll. In Abbildung 2.20 sind die Ergebnisse dieser Messungen dargestellt.

In der Tabelle 2.2 sind die gemessenen und die daraus bestimmten Parameter aufgetragen. Insbesondere stehen für die schmalbandige Beleuchtung der Probe $2,9 \cdot 10^{8}$ Photonen pro Laserpuls bei senkrechtem Einfall auf eine Fläche von $60 \times 95 \mu^{2}(\mathrm{FWHM})$ zur Verfügung. Nach einer Belichtung über 2000 Pulse wäre damit eine Mindestzahl von Photonen zur Erzeugung eines mikroskopischen Bildes erreicht. 


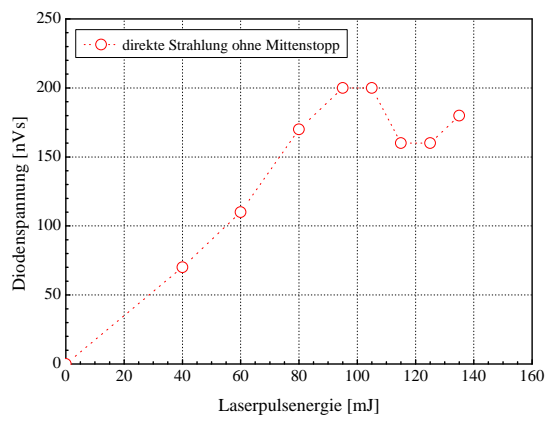

(a) Direkte durch eine $650 \mu \mathrm{m}$ Lochblende an Probenposition detektierte XUVStrahlung (breitbandig).

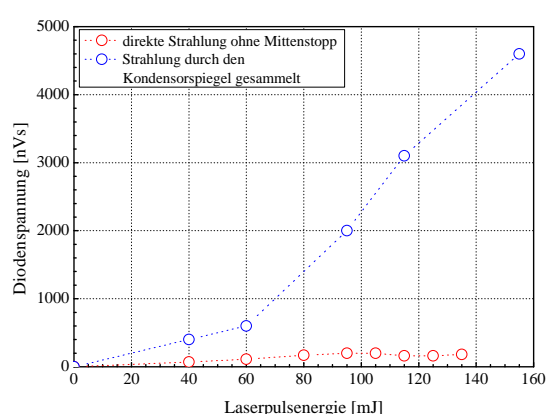

(b) Vom Kondensorspiegel reflektierte XUV-Strahlung. Die direkte Strahlung wird durch den Mittenstopp geblockt.

Abbildung 2.20:

Messung der Photonendichte an der Probenposition mit einer kalibrierten AXUV-100-Photodiode (IRD Inc.)

Tabelle 2.2:

Photonenfluss der Laserplasmaquelle mit kryogenen Argonstrahl als Target an der Probenposition mit einer kalibrierten AXUV-100-Photodiode (IRD Inc.).

\begin{tabular}{|c|c|c|}
\hline & $\begin{array}{l}\text { Direktes Licht des } \\
\text { Plasmas durch ein } \\
650 \mu \mathrm{m} \text { Pinhole }\end{array}$ & $\begin{array}{l}\text { Reflektiertes Licht } \\
\text { des Cr/Sc-Spiegels }\end{array}$ \\
\hline $\begin{array}{l}\text { Mittlere Diodenspannung (Mittel } \\
\text { über } 5 \text { Pulse) }\end{array}$ & $200 \cdot 10^{-9} \mathrm{Vs}$ & $1000 \cdot 10^{-9} \mathrm{Vs}$ \\
\hline $\begin{array}{l}\text { Resultierender Diodenstrom (Mittel } \\
\text { über } 5 \text { Pulse) }\end{array}$ & $2 \cdot 10^{-10} \mathrm{As}$ & $1 \cdot 10^{-9} \mathrm{As}$ \\
\hline Elektronenanzahl & $1,25 \cdot 10^{9}$ & $6,25 \cdot 10^{9}$ \\
\hline Quanteneffizienz AXUV-100 Diode & $\approx 100 \frac{\text { Elektronen }}{\text { Photon }}$ & $\approx 100 \frac{\text { Elektronen }}{\text { Photon }}$ \\
\hline Resultierende Photonenanzahl & $1,25 \cdot 10^{7}$ & $2,9 \cdot 10^{8}$ \\
\hline Beobachteter Raumwinkel & $3,344 \cdot 10^{-6} \mathrm{sr}$ & $0,027 \mathrm{sr}$ \\
\hline Photonenanzahl in $4 \pi$ & $4,5 \cdot 10^{13} \frac{\text { Photonen }}{\text { Puls }}$ & $1,3 \cdot 10^{13} \frac{\text { Photonen }}{\text { Puls }}$ \\
\hline Photonenfluss & $3,6 \cdot 10^{12} \frac{\text { Photonen }}{\text { sr.Puls }}$ & $1,0 \cdot 10^{12} \frac{\text { Photonen }}{\text { sr.Puls }}$ \\
\hline
\end{tabular}




\subsection{Fazit}

Das Quellkonzept eines flüssigen Argonstrahls als Laserplasmatarget für den Aufbau eines Laborröntgenmikroskops erreicht einen Photonenfluss, der in der gleichen Größenordnung liegt wie andere aktuelle schmalbandige und hochbrillante XUV-Strahlungsquellen (siehe beispielsweise [Bertilson u. a., 2011]). Durch den Einsatz eines breitbandig emittierenden Targets lässt sich jedoch durch die Kondensoroptik eine oder mehrere Wellenlängen selektieren, sodass über die Absorptionskanten elementspezifische Mikroskopie möglich wird.

Die experimentellen Herausforderungen, die ein so aufwendiges Targetkonzept bedingt, sind jedoch erheblich. Diese sind vor allem Verschmutzung und Verstopfung der Kapillaren, Lagestabilität des Argonstrahls während der Plasmazündung und Beschädigungen empfindlicher Experimentteile durch Debris-Teilchen. Der Einsatz eines optimierten, debris-freien Gastargets, bietet aufgrund seiner Zuverlässigkeit und Lagestabilität, trotz geringer Repetitionsrate und Brillanz, zum derzeitigen Entwicklungsstand eine Alternative zu dem kryogenen Argontarget. 


\section{Kapitel 3}

\section{Charakterisierung von Multischicht-Laue-Linsen}

\section{Einleitung}

Um hochaufgelöste mikroskopische Bilder zu erzeugen, bedarf es leistungsfähiger Optiken, die eine sehr kleine minimale Strahltaillenbreite ermöglichen. Die dazu notwendige Charakterisierung der ein- und zweidimensionalen Strahltaillen und Foki im Bereich von $10-100 \mathrm{~nm}$ soll an einer Laserplasmaquelle (gasförmiges Stickstofftarget $\lambda=2,88 \mathrm{~nm}$ ) für weiche Röntgenstrahlung durchgeführt werden. Als eine solche Optik wurde die Strahlcharakteristik einer Multischicht-Laue-Linse (MLL, englisch: „Multilayer Laue Lens") vermessen. Eine Simulation dieses Experiments liefert detaillierten Einblick in die Strahlausbreitung und wird mit Messergebnissen und Designparametern verglichen.

\subsection{Herstellung}

Das Design und die Herstellung der Multischicht-Laue-Linse wurde von Tobias Liese am Institut für Materialphysik der Universität Göttingen durchgeführt. In dieser Arbeit soll nur ein Überblick über den Herstellungsprozess gegeben werden. Eine ausführliche Darstellung findet sich in Liese u. a. [2010, 2011]. Zur Herstellung der Probe wurde eine

\footnotetext{
${ }^{3}$ Die Herstellung der Multischicht-Laue-Linsen wurde von Tobias Liese am Institut für Materialphysik der Universität Göttingen (UMP) geplant und durchgeführt. Die Proben wurden anschließend im Rahmen dieser Arbeit am Laser-Laboratorium Göttingen e.V. (LLG) charakterisiert und simuliert. Die Messungen zur Bestimmung des Divergenzwinkels am BESSY II Speicherring wurden von Dr. Dong-Du Mai in Kooperation mit der Arbeitsgruppe Dr. Rosenhahn der Universität Heidelberg durchgeführt.

Im Rahmen des Projektes C4 innerhalb des Sonderforschungsbereichs 755 „Nanoscale Photonic Imaging" entstanden neben dieser Arbeit zum Thema der Herstellung und Charakterisierung von Multischicht-Laue-Linsen die folgenden Publikationen: Herstellungsverfahren der MLL durch das UMP [Liese, Radisch und Krebs, 2010], Publikation erster Ergebnisse zur Entwicklung (UMP) und Charakterisierung (LLG in Kooperation mit dem UMP) [Liese, Radisch, Knorr, Reese, Großmann, Mann und Krebs, 2011] und ausführliche Charakterisierung (LLG mit Unterstützung durch das UMP) [Reese, Schäfer, Großmann, Bayer, Mann, Liese und Krebs, 2011].
} 
neue Kombination von gepulster Laserdeposition (PLD, engl. „pulsed laser deposition“) und fokussiertem Ionenstrahl (FIB, engl. ,focussed ion beam") verwendet. Die dabei angewendeten Techniken werden im Folgenden vorgestellt anschließend wird die Fertigung der eigentlichen Proben präsentiert.

\subsubsection{Motivation}

Die Erzugung immer kleinerer Strukturen bei der Fertigung von Fresnel'schen Zonenplatten (Kapitel 1.3.2) für weiche Röntgenstrahlung gestaltet sich mit den klassischen Herstellungsmethoden zunehmend schwieriger. Mittels eines Verfahrens, welches Elektronenstrahllithografie, Ionenätzen und Überlagerungstechniken verbindet, können die bisher kleinsten Strukturen im Bereich von $12 \mathrm{~nm}$ erzeugt und damit Abbildungsleistungen der gleichen Größenordnung erzeugt werden [Chao u.a., 2005, 2009a]. Diese Technologie der Herstellung scheint allerdings mehr und mehr an die Grenzen der technischen Machbarkeit zu stoßen, da die notwendigen zu erreichenden Aspektverhältnisse zwischen Spalten und Stegen immer größer werden.

Als alternativer Ansatz kann zur Erzeugung solcher diffraktiver Optiken stattdessen ein Vielschichtsystem hergestellt, geeignet geschnitten, geschliffen und poliert werden um damit die gewünschten diffraktiven Eigenschaften zu erzeugen [Tamura u. a., 2002]. Diese Technik hat den Vorteil, dass die Zonenplatten in praktisch beliebiger Dicke hergestellt werden können, um das notwendige Aspektverhältnis für die erforderliche Absorption oder den benötigten Phasenschub zu erreichen. Ferner können die Effizienzen der Optik durch geeignete Deposition, Materialwahl und Fertigung zusätzlich durch keilförmige Ausführung der Schichten [Conley u. a., 2008] oder das Nachbilden der Kinoform der Fresnellinse [Tamura u. a., 2009] optimiert werden. Das Aufbringen von Schichten, die deutlich dünner sind als die Strukturen aus elektronenstrahllithographischer Herstellung, ist mittels Pulsed Laser Deposition möglich, wobei das systematische Glätten von kumulativen Rauigkeiten geeigneter Schichtsysteme ausgenutzt wird [Röder, 2009; Röder u.a., 2010]. Anstelle des mechanischen Schneidens und Polierens des Schichtpakets [Kang u. a., 2007] kommt hier ein fokussierter Ionenstrahl zum Einsatz, der deutlich weniger Störungen in den Schichtpaketen verursacht. Durch die Kombination dieser beiden Techniken können die Limitierungen der lithographischen Herstellung von Zonenplatten in Strukturgröße und Aspektverhältnis umgangen werden. Multischicht-Laue-Linsen wurden bisher für harte Röntgenstrahlung demonstriert und $\mathrm{WSi}_{2} / \mathrm{Si}-\mathrm{Multischichten} \mathrm{er-}$ reichen einen Linienfokus von $16 \mathrm{~nm}$ bei 19,5 keV [Kang u. a., 2008].

\subsubsection{Pulsed Laser Deposition}

Zum Auftragen der Schichten der Multischicht-Laue-Linse wurde das PVD-Verfahren (PVD, englisch: physical vapour deposition - physikalische Gasabscheidung) der gepuls- 
ten Laserdeposition (PLD, englisch: pulsed laser deposition) angewendet, das hier kurz erläutert werden soll. „Die gepulste Laserdeposition [...] ist eine vielseitige Dünnschichtmethode, mit der sich nicht nur Metalle, Keramiken oder Oxide deponieren lassen, sondern auch so komplexe Materialien wie Polymere. Ein Vorteil dieser Methode ist ein im Allgemeinen stöchiometrischer Materialübertrag zwischen Substrat und Target." [Röder, 2009] Dazu wird ein Laser auf einen Festkörper fokussiert, welcher sich dadurch lokal stark erhitzt und abgetragen wird. Bei dem für die Herstellung der Schichtpakete verwendeten PLD-Standardaufbau [Krebs und Bremert, 1993] wurde der Strahl eines Excimer-Lasers (Lambda Physik LPX 110i, 248 nm Wellenlänge, 30 ns Pulsdauer, $5 \frac{\mathrm{J}}{\mathrm{cm}^{2}}$ Energiedichte bei $10 \mathrm{~Hz}$ ) in einer Vakuumkammer auf das zu deponierende Substratmaterial bei Zimmertemperatur fokussiert. Der dabei erreichte Arbeitsdruck in der Vakuumkammer lag stets unter $10^{-8}$ mbar. Das Substratmaterial wird dabei so stark erhitzt, dass eine „Plasmakeule“ entsteht, deren Materialstrom das Target trifft und sich dort ablagert. Auf diese Weise kann das Material in sehr homogenen, dünnen und ebenen Schichten aufgetragen werden.

\subsection{3 fokussierter lonenstrahl}

Zur Bearbeitung und Analyse der hergestellten Vielschichtsysteme kam ein fokussierter Ionenstrahl (FIB, englisch: focussed ion beam) zum Einsatz. Mit dem dabei eingesetzten Gallium-Ionenstrahl lässt sich eine große Spannweite von Materialien vergleichbarer oder leichterer Massenzahlen bis in den Bereich der Nanometerskala abbilden und bearbeiten [Hayles u. a., 2009]. Der Aufbau eines solchen Geräts ist, bis auf den wesentlichen Unterschied, dass statt der Elektronenkanone eine flüssige Metallquelle und anstelle der sonst üblichen magnetischen Linsen elektrostatische Linsen verwendet werden, weitgehend analog zu den bekannten Elektronenstrahlmikroskopen. Diese Variation ist notwendig, da die Lorenzkraft nur schwach auf die relativ langsamen Ionen wirkt. Durch die Größe der Ionen - verglichen mit der Masse der Elektronen - treten sie in intensive Wechselwirkung mit dem Material, auf das sie fokussiert werden. Durch die vergleichbare Masse der verwendeten Gallium-Atome mit den Atomen des Targets können diese aus ihrer Matrix gestoßen und anschließend im Vakuum desorbiert werden. Ein geringer Anteil der bei diesem Prozess zusätzlich ionisierten Targetatome kann dabei zur Erzeugung eines Bildes eingesetzt werden. Durch Kontrolle der Bestrahlungsdauer und des Bestrahlungsstroms kann sehr genau Material abgetragen und so auch sehr kleine Strukturen erzeugt werden. Durch Einleiten von Methyl-cyclo-pentadienyl-Pt(IV)-tri-methyl $\left(\mathrm{CH}_{3}\right)_{3} \mathrm{Pt}\left(\mathrm{C}_{\mathrm{p}} \mathrm{CH}_{3}\right)$ in den Gallium-Ionen- oder Elektronenstrom lässt sich gezielt in kleinen Bereichen Platin deponieren. Das bei der Herstellung genutzte Gerät FEI Nova Nanolab ${ }^{\circledR}$ verfügt außerdem über ein Rasterelektronenmikroskop und einen Mikromanipulator (Omniprobe $\left.{ }^{(}\right)$), mit dem sich die Probe im Gerät bewegen lässt. 


\subsubsection{Fertigung der Multischicht-Laue-Linse}

Mit den beiden oben beschriebenen Verfahren wurden die Proben aus einem Vielschichtsystem hergestellt und anschließend so bearbeitet, dass sie als Optik für weiche Röntgenstrahlung benutzt werden können. Ein $\mathrm{Ti} / \mathrm{ZrO}_{2}$ Schichtsystem wird dazu mit dem in Kapitel 3.1.2 beschriebenen Versuchsaufbau auf ein Silizium(111)-Substrat aufgetragen. Als Schichtsysteme kamen Titan oder Magnesiumdioxid als transmittierendes und Zirkoniumoxid als optisch absorbierendes und gleichzeitig schichtglättendes Material zum Einsatz. Die Depositionsraten der Materialien wurden mit einem Profilometer bestimmt. Die Fertigung unter dem fokussierten Ionenstrahl ist in Abbildung 3.1 dargestellt.

Die hergestellten Multischichten wurden unter einer dünnen Schicht Platin, aufgebracht mittels des Elektronenstrahls, geschützt. Anschließend wurde diese Schicht unter Einsatz des gröberen Ionenstrahls auf eine Schicht von 5,1 $\mu \mathrm{m}$ vergrößert. Danach wurde das Schichtpaket in einer Dicke von $1 \mu \mathrm{m}$ freigestellt. Dieses Paket wurde herausgelöst, durch den Mikromanipulatur an eine Kupferlamelle befestigt und dort mit deponiertem Platin fixiert. Ebenso wurde mit einem zweiten Paket verfahren, das, um $180^{\circ}$ Grad gedreht, gegenüber dem ersten Paket befestigt wurde. Mit Hilfe des Rasterelektronenmikroskops wurde der Abstand der Schicht exakt ausgerichtet. Die notwendige Genauigkeit liegt dabei im Bereich der kleinsten Zonenbreite. Bei der ersten hergestellten Probe mit zwei gegenüberliegenden Schichtpaketen wurde diese Genauigkeit jedoch aus apparativen Gründen noch nicht erreicht und lag etwa $1 \mu \mathrm{m}$ von der optischen Achse entfernt. Der hierbei belassene Spalt wurde durch Deposition von Platin geschlossen, welches später auch als Strahlstopp verwendet wurde. Diese Multischicht-Laue-Linse wurde anschließend durch einen geringen Ionenstrom auf die korrekte Dicke gedünnt und anschließend poliert. Zuletzt wurde sie in eine Streulicht unterbindende Aperturblende gelegt, die ebenfalls mittels des Ionenstrahls in eine mit $530 \mathrm{~nm}$ Gold bedampfte $\mathrm{Si}_{3} \mathrm{~N}_{4}$-Folie (Silson Ltd.) gebohrt wurde.

Bei der hergestellten Dicke der Multischicht-Laue-Linse von etwa $200 \mathrm{~nm}$ bei Probe 1 und etwa $150 \mathrm{~nm}$ bei Probe 2 werden $85 \%$ beziehungsweise $77 \%$ der XUV-Strahlung in der absorbierenden $\mathrm{ZrO}_{2}$-Schicht abgefangen, während $72 \%$, respektive $88 \%$ in der $\mathrm{Ti}$ - $/ \mathrm{MgO}_{2}$-Schicht transmittiert werden. Die durch beide Schichten transmittierte XUVStrahlung hat einen Phasenversatz von 0,95 rad bei Probe 1a/b und 0,55 rad bei Probe 2. In erster Näherung handelt es sich in beiden Fällen um eine absorbierende Zonenplatte. Durch die endliche Dicke der Zonenplatte und die phasenverschobene Transmission im absorbierenden Material kann eine Abnahme der Effizienz der Multischicht-LaueLinse erwartet werden [Kopylov u. a., 1995]. In der Tabelle 3.1 werden die Parameter der beiden hergestellten Proben zusammengefasst. Zusätzlich ist eine simulierte, aber nicht hergestellte Linse aufgeführt. Die fertige Multischicht-Laue-Linse in ihrer Aperturblende ist in Abbildung 3.2 als Bild unter dem Rasterelektronenmikroskop dargestellt. 
Tabelle 3.1:

Designparameter der in den Experimenten verwendeten Multischicht-Laue-Linse. Es handelt sich dabei um Schichtpakete, die einen Linienfokus erzeugen. Die hergestellten Proben sind „Off-Axis Zonenplatten“, die nur ein Teilbereich der Fresnelschen Zonenplatte sind. Die Volllinse ist nicht gefertigt worden, wird aber in Kapitel 3.3.7 simuliert.

\begin{tabular}{|c|c|c|c|c|c|}
\hline & $\begin{array}{l}\text { Wellen- } \\
\text { länge } \lambda\end{array}$ & $\begin{array}{l}\text { Brenn- } \\
\text { weite } f\end{array}$ & $\begin{array}{l}\text { kleinste } \\
\text { Zone } \# Z_{1}\end{array}$ & $\begin{array}{c}\text { größte } \\
\text { Zone \# } Z_{2}\end{array}$ & $\begin{array}{l}\text { Abstand } \\
\text { zur } \\
\text { optischen } \\
\text { Achse } m\end{array}$ \\
\hline $\begin{array}{l}\text { MLL Probe 1a } \\
2 \text { Schichtpakete }\end{array}$ & $2,88 \mathrm{~nm}$ & $250 \mu \mathrm{m}$ & 29 & 113 & $4568 \mathrm{~nm}$ \\
\hline $\begin{array}{l}\text { MLL Probe 1b } \\
1 \text { Schichtpaket }\end{array}$ & $2,88 \mathrm{~nm}$ & $250 \mu \mathrm{m}$ & 29 & 113 & $4568 \mathrm{~nm}$ \\
\hline MLL Probe 2 & $2,88 \mathrm{~nm}$ & $100 \mu \mathrm{m}$ & 605 & 705 & $13197 \mathrm{~nm}$ \\
\hline \multirow[t]{2}{*}{ MLL Volllinse } & $2,88 \mathrm{~nm}$ & $250 \mu \mathrm{m}$ & 1 & 200 & $0 \mathrm{~nm}$ \\
\hline & $\begin{array}{l}\text { dünnste } \\
\text { Schicht }\end{array}$ & $\begin{array}{l}\text { dickste } \\
\text { Schicht }\end{array}$ & Dicke & $\begin{array}{l}\text { Apertur } \\
\quad a \times b\end{array}$ & $\begin{array}{c}\text { Material- } \\
\text { system }\end{array}$ \\
\hline $\begin{array}{l}\text { MLL Probe 1a } \\
2 \text { Schichtpakete }\end{array}$ & $40 \mathrm{~nm}$ & $79,5 \mathrm{~nm}$ & $\approx 200 \mathrm{~nm}$ & $\begin{array}{l}3980 \mathrm{~nm} \\
\times 16 \mu \mathrm{m}\end{array}$ & $\mathrm{Ti} / \mathrm{ZrO}_{2}$ \\
\hline $\begin{array}{l}\text { MLL Probe } 1 \mathrm{~b} \\
1 \text { Schichtpaket }\end{array}$ & $40 \mathrm{~nm}$ & $79,5 \mathrm{~nm}$ & $\approx 200 \mathrm{~nm}$ & $\begin{array}{l}3980 \mathrm{~nm} \\
\times 16 \mu \mathrm{m}\end{array}$ & $\mathrm{Ti} / \mathrm{ZrO}_{2}$ \\
\hline $\begin{array}{l}\text { MLL Probe } 2 \\
2 \text { Schichtpakete }\end{array}$ & $10,11 \mathrm{~nm}$ & $10,91 \mathrm{~nm}$ & $\approx 150 \mathrm{~nm}$ & $\begin{array}{l}1005 \mathrm{~nm} \\
\times 19 \mu \mathrm{m}\end{array}$ & $\mathrm{MgO}_{2} / \mathrm{ZrO}_{2}$ \\
\hline MLLVolllinse & $30 \mathrm{~nm}$ & $848 \mathrm{~nm}$ & & $23994 \mathrm{~nm}$ & \\
\hline
\end{tabular}



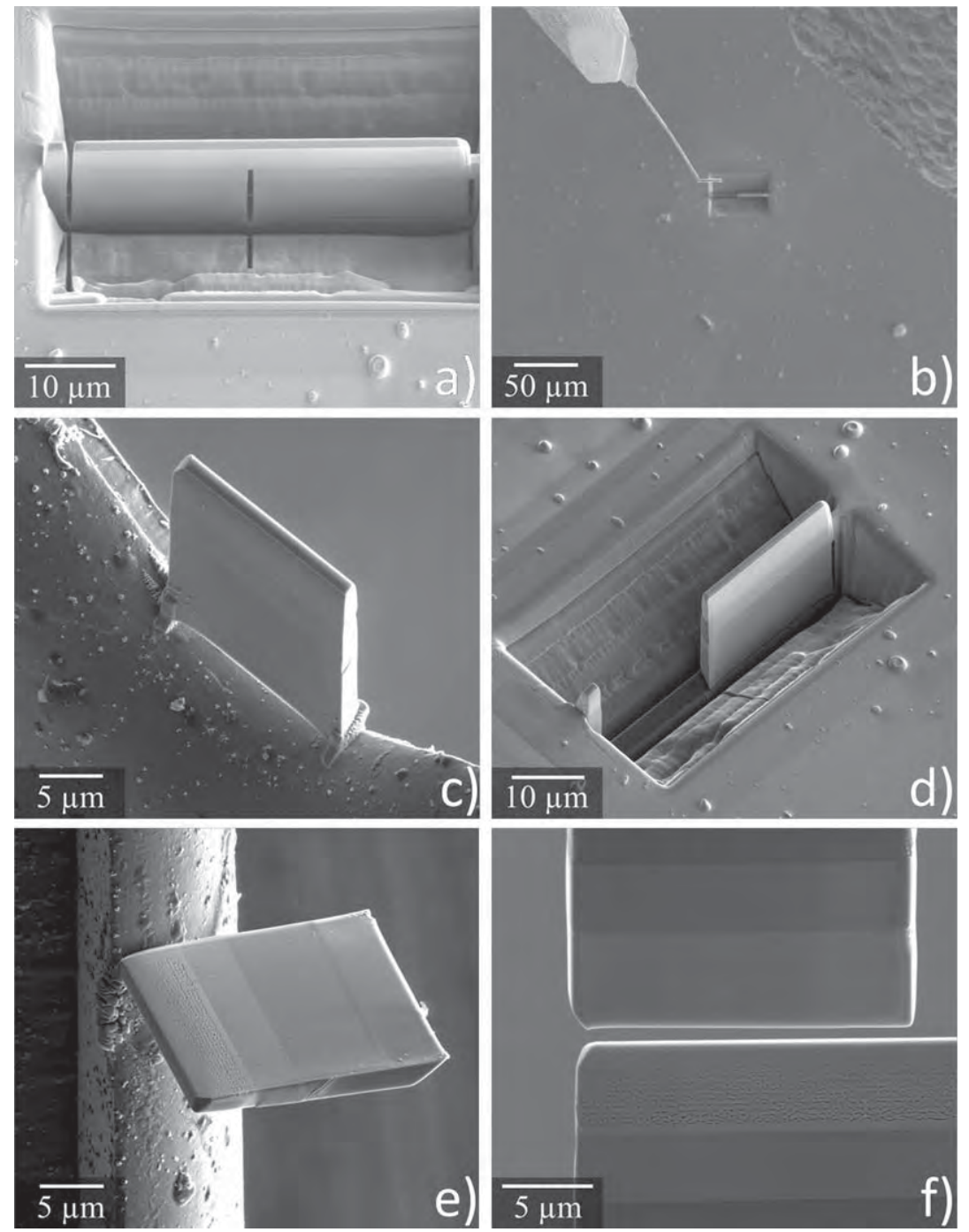

Abbildung 3.1:

Probe 1: Rasterelektronenmikroskopische Aufnahmen der einzelnen Schritte des Herstellungsprozesses der Multischicht-Laue-Linse unter dem fokussiertem lonenstrahl durch Liese u. a. [2010]. (a) Unter Platin verborgenes und anschließend freigestelltes Schichtpaket, (b) Herauslösen eines Schichtpakets mit dem Mikromanipulator, (c) auf einer Kupferlamelle befestigtes Schichtpaket, (d) verbleibendes zweites Schichtpaket, (e) halb rotiertes zweites Schichtpaket, (f) zueinander zum Doppelpaket ausgerichtete Schichtpakete. 


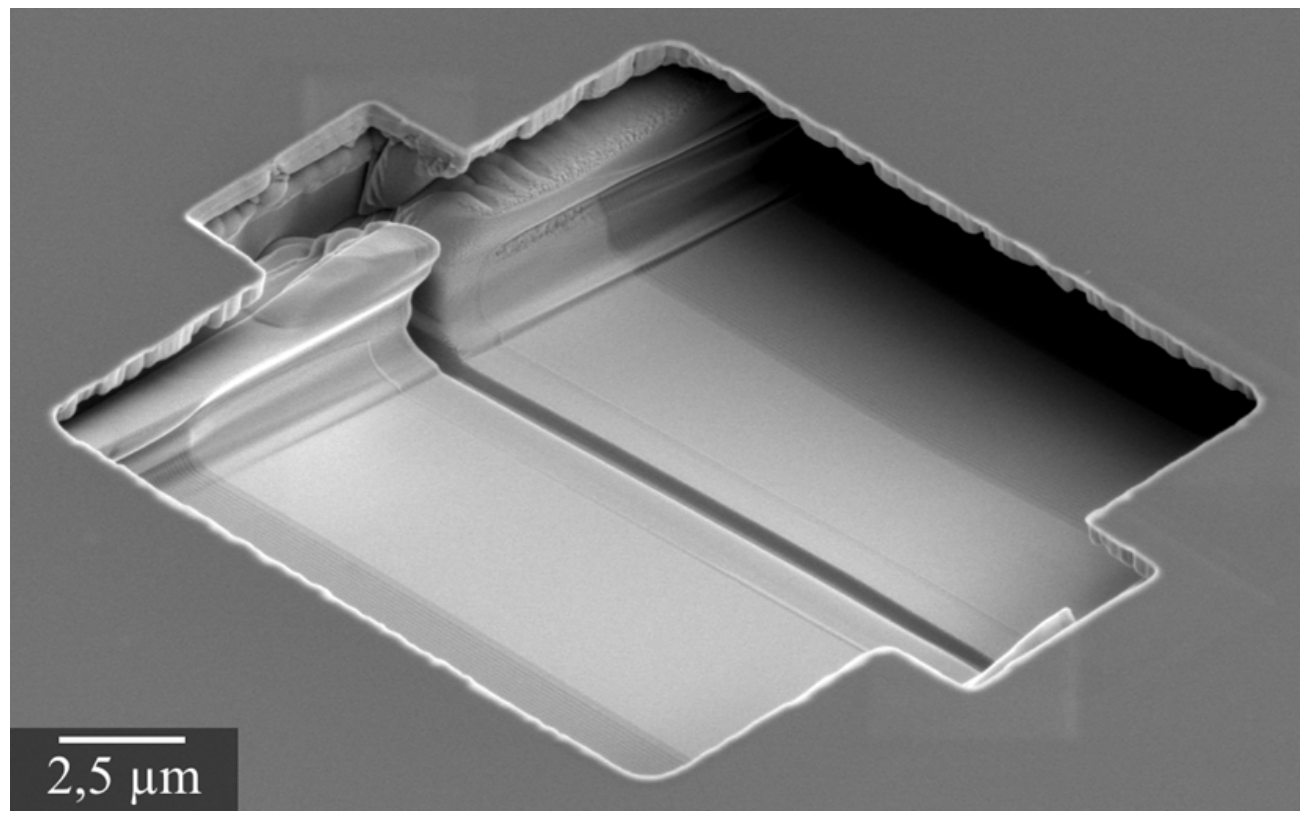

Abbildung 3.2:

Probe 1a: Die mit Platin zusammengefügten und in eine Aperturblende eingebetteten Schichtpakete. Durch diesen Probenaufbau wird verhindert, dass im Experiment Streulicht an der Optik vorbei gelangt und die Messung stört. [Liese u. a., 2010] 


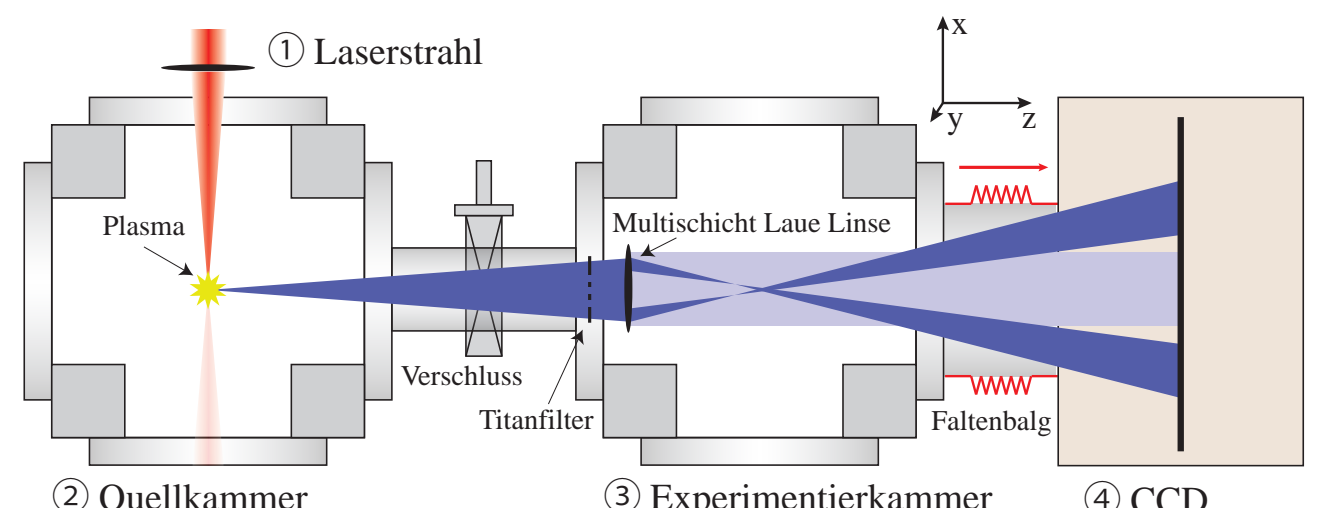

(2) Quellkammer

(3) Experimentierkammer

(4) CCD

\section{Abbildung 3.3:}

Der Aufbau des Divergenzwinkelexperiments. Der Laserstrahl (1) zündet ein Plasma in der Quellkammer (2). Das emittierte Spektrum des Stickstoffplasmas wird durch einen Titanfilter monochromatisiert und fällt auf die Multischicht-Laue-Linse in der Probenkammer (3). Die Beugungsordnungen der Linse werden anschließend von der verfahrbaren CCD-Kamera (4) in verschiedenen Abständen registriert.

\subsection{Experimentelle Strahlcharakterisierung}

Im Folgenden werden die Experimente zur Bestimmung der optischen Eigenschaften der Schichtpakete beschrieben. Hierzu wurden ein Divergenzwinkel- und eine Schneidblendenrastermessung durchgeführt.

\subsubsection{Divergenzwinkelexperiment}

Als erster Ansatz wurde ein Experiment zur Bestimmung des Divergenzwinkels der Beugungsordnungen durchgeführt. Eine schematische Zeichnung zum Versuchsaufbau findet sich in Abbildung 3.3. Wegen der hohen Absorption von weicher Röntgenstrahlung durch Luft muss sich der Aufbau in einer evakuierten Kammer befinden. Die in Kapitel 2.1 auf Seite 24 beschriebene Laserplasmaquelle wurde mit einem Nd:YAG-Laser (Innolas YMR800, ausgewählte Parameter finden sich in Tabelle 2.1 auf Seite 26) betrieben. Als Plasmatargetmaterial kam dabei gasförmiger Stickstoff zum Einsatz, der ein Linienspektrum emitiert. Das Targetgas wurde durch ein Proch-Trickl-Ventil synchronisiert zum Laserstrahl in die Kammer geleitet [Proch und Trickl, 1989]. Durch Filterung dieser Strahlung mit einer $200 \mathrm{~nm}$ Titanfolie kann eine einzelne Wellenlänge von 2,88 nm isoliert werden. Diese monochromatisierte Strahlung des isotropen Kugelstrahlers kann bei dem großen Quellabstand von $80 \mathrm{~cm}$ und der kleinen Aperturgröße (Tabelle 3.1) als annähernd paralleles Strahlenbündel mit einer beinahe ebenen Wellenfront angesehen werden. Da der Quellabstand verglichen mit der Brennweite $f$ des fokussierenden Schichtpakets sehr groß ist, wird sie praktisch exakt in den Fokus abgebildet. Der 


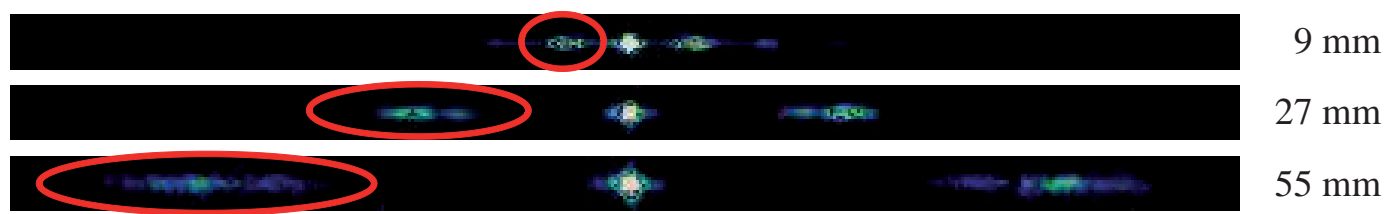

Abbildung 3.4:

Beispiele der beim Divergenzwinkelexperiment aufgenommenen Detektorbilder. Der Abstand von Probe 1 wurde an Hand des extrapolierten Schnittpunkts der Beugungsordnungen bestimmt. Mit den roten Einkreisungen sind die zur Strahlbreitenbestimmung ausgewerteten relevanten Bildbereiche gekennzeichnet. Es zeigt sich, dass die Beugungsordnungen auseinanderlaufen und sich in sich verbreitern.

Verkleinerungsmaßstab ist dabei so groß, dass die nominelle Bildpunktgröße die Abbildungsleistung der Proben 1 und 2 unterschreitet. Hinter dem Bildpunkt divergiert der Stahl wieder. In einer geringeren Entfernung von 2-10 cm hinter dem Schichtpaket wurden die verschiedenen Beugungsordnungen von einer rückseitig gedünnten CCD-Kamera (Roper Scientific PI-SX:1k, $1024^{2}$ Pixel, $13 \times 13 \mu \mathrm{m}^{2}$ Pixelgröße) detektiert. Diese kann mit einem Verfahrtisch und einem Faltenbalg entlang der optischen Achse positioniert werden. Durch Integration der Detektorereignisse über 1200 Pulse bei $1 \mathrm{~Hz}$ Repetitionsrate wurde ein gut ausgesteuertes Signal auf dem Detektor erreicht. Exemplarisch sind einige der Detektorbilder in Abbildung 3.4 dargestellt. Dabei ist die transmittierte 0. und die fokussierende 1. Beugungsordnung besonders deutlich zu erkennen. Die auch im Fernfeld zu erkennende starke Feinstrukturierung des Strahlprofils deutet schon zu diesem Zeitpunkt auf einen stark aberrierten Strahl hin. Eine genauere Untersuchung dieser Strahlcharakteristik wird in der Simulation in Kapitel 3.3.5 erfolgen.

\section{Bestimmung der Brennweite aus der Lage der Beugungsordnungen}

Zur Bestimmung der Strahlparameter kann die Lage der 0. und der 1. Beugungsordnung zueinander, aber auch die Verbreiterung der fokussierenden 1. Beugungsordnung, dem Divergenzwinkel, ausgewertet werden. In Abbildung 3.5 sind zwei Betrachtungen der geometrischen Strahloptik dargestellt, mit denen sich die Brennweite der MultischichtLaue-Linse aus den Messwerten und den Probenparametern berechnen lässt.

Aus ihnen lassen sich unter der Annahme kleiner Winkel, wie sie im Experiment vorliegen, die folgenden beiden Zusammenhänge für die Brennweite $f$ herleiten:

$$
\begin{aligned}
\frac{x}{d} & =\frac{m+a / 2}{f}=\tan (\beta) \\
\Rightarrow f & =\frac{d \cdot(m+a / 2)}{x},
\end{aligned}
$$




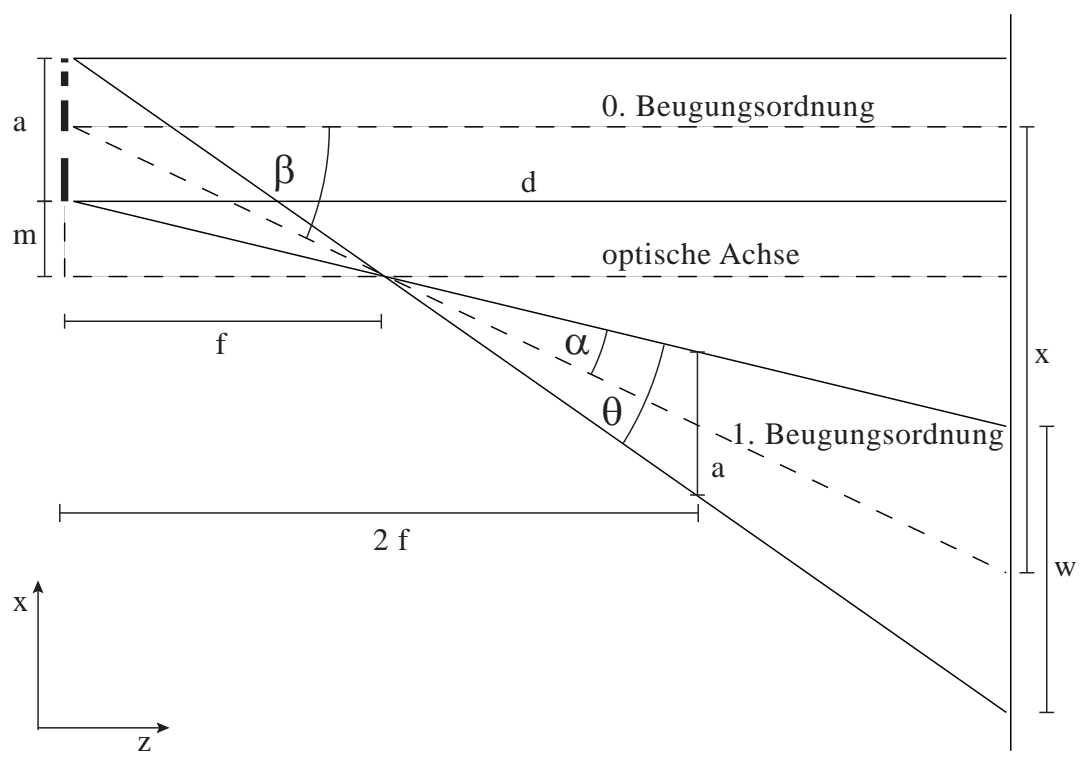

\section{Abbildung 3.5:}

Strahloptische Überlegung zum Divergenzwinkelexperiment. Hinter einem einzelnen Schichtpaket breiten sich die einzelnen Beugungsordnungen in verschiedene Richtungen aus. Von besonderem Interesse sind hierbei die transmittierte 0 . und die fokussierende 1. Beugungsordnung. Aus den Winkelbeziehungen der beiden zueinander, aber auch der Divergenz der 1. Ordnung lässt sich über einfache geometrische Beziehungen die Brennweite berechnen.

sowie

$$
\begin{aligned}
\frac{a / 2}{f} & =\frac{w / 2-a / 2}{d-2 f} \\
\Rightarrow f & =\frac{d \cdot a / 2}{w / 2+a / 2} .
\end{aligned}
$$

Die Größe des sich im Abstand $m$ von der optischen Achse befindlichen Schichtpakets der Aperturgröße $a$ und die zu überprüfende Brennweite $f$ werden bei der Herstellung des Schichtpakets festgelegt. Der Abstand der Beugungsmaxima $x$ zueinander und die Breite der fokussierten Beugungsordnung $w$ in Abhängigkeit des Abstands $d$ des CCDDetektors von dem Schichtsystem werden im Experiment bestimmt. Zur Auswertung der Strahlbreiten kommt die Software ImageJ zum Einsatz [Rasband, 1997 - 2011; Abramoff u. a., 2004].

Aus den ermittelten Strahlbreiten lässt sich durch Regression auch der Divergenzwinkel $\Theta$, in Abbildung 3.6 dargestellt, genau bestimmen. Die Ergebnisse dieser Auswerung sind in Tabelle 3.4 auf Seite 71 zusammengefasst und zeigen eine gute Übereinstimmung mit dem Vergleichsexperiment am BESSY II (Kapitel 3.2.3) beziehungsweise der Simulation einer fehlerbehafteten Probe. 


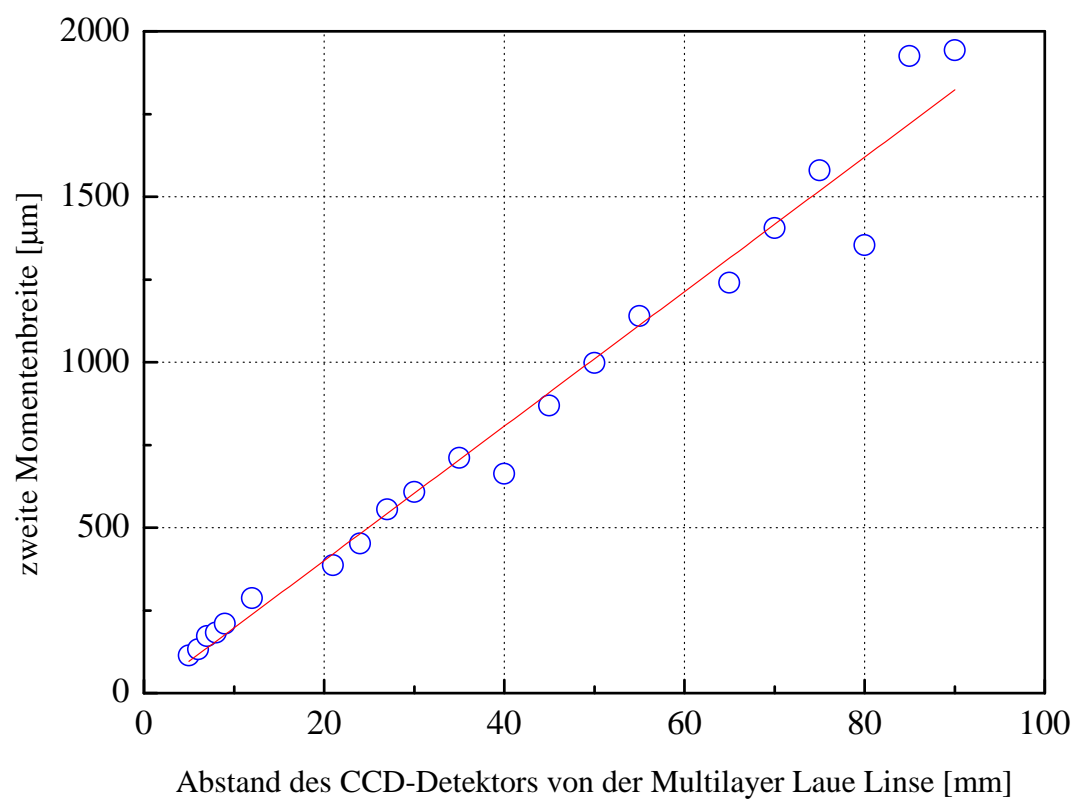

Abbildung 3.6:

Über die im Divergenzwinkelexperiment gemessenen Strahlbreiten über dem Abstand zur Multischicht-LaueLinse lässt sich durch lineare Regression der Divergenzwinkel $\Theta$ der fokussierenden Beugungsordnung bestimmen.

\section{Strahlparameterbestimmung mit Gaußstrahloptik}

Die Grundlage für einen alternativen Ansatz zur Bestimmung der Brennweite $f$ bildet die Gaußsche Strahloptik (Anhang A). Hierbei wird diese aus dem Divergenzwinkel und der zweiten Momentenbreite der Aperturgröße $w_{\text {Apertur }}$ über die Rayleighlänge $z_{\mathrm{R}}$ bestimmt. Dabei wird auch hier vorausgesetzt, dass sich der Strahl symmetrisch vor und hinter dem Bildpunkt ausbreitet und nur gering aberriert ist:

$$
\begin{aligned}
\Theta & =\arctan \left(\frac{w}{z}\right)=\arctan \left(\frac{\lambda}{\pi w_{0}}\right) \\
& \Rightarrow w_{0}=\frac{\lambda}{\pi \cdot \tan (\Theta)} \\
z_{\mathrm{R}} & =\frac{\pi \cdot w_{0}^{2}}{\lambda} \\
w & =w_{0} \sqrt{1+\left(\frac{z}{z_{\mathrm{R}}}\right)^{2}} \\
& \Rightarrow z(f)=z_{\mathrm{R}} \cdot \sqrt{\left(\frac{w_{\text {Apertur }}}{w_{0}}\right)^{2}-1}
\end{aligned}
$$


Tabelle 3.2:

Ergebnisse der Divergenzwinkelmessung und Abschätzungen der Brennweiten. Für MLL 1 zeigen sich große Abweichungen der Methoden, die allerdings durch die herstellungsbedingten Aberrationen erklärt werden. Die Breite des fokussierenden Beugungsmaximums von MLL 2 konnte aufgrund ihres sehr schwachen Signals nur schlecht bestimmt werden. Daher wurde die Brennweite nicht aus der Verbreiterung der 1. Beugungsordnung oder der Methode der Gaußstrahloptik bestimmt.

\begin{tabular}{llllll}
\hline & $\begin{array}{l}\text { Design- } \\
\text { brennweite }\end{array}$ & $\begin{array}{l}\text { Brennweite } \\
\text { aus Beu- } \\
\text { gungswinkel } \\
\beta\end{array}$ & $\begin{array}{l}\text { Divergenz- } \\
\text { winkel } \theta\end{array}$ & $\begin{array}{l}\text { Brennweite } \\
\text { aus Strahl- } \\
\text { optik }\end{array}$ & $\begin{array}{l}\text { Brennweite } \\
\text { aus Gauß- } \\
\text { strahl }\end{array}$ \\
\hline MLL Probe 1 & $250 \mu \mathrm{m}$ & $177 \mu \mathrm{m}$ & $\begin{array}{l}20,4 \pm 0,3 \\
\text { mrad }\end{array}$ & $224 \mu \mathrm{m}$ & $220 \mu \mathrm{m}$ \\
MLL Probe 2 & $100 \mu \mathrm{m}$ & $98 \mu \mathrm{m}$ & & & \\
\hline
\end{tabular}

Die Ergebnisse dieser Berechnungen werden zusammenfassend in Tabelle 3.2 dargestellt. $\mathrm{Zu}$ beachten gilt, dass die angegebenen Ergebnisse sehr sensibel auf einige der beobachteten Aberrationen der Schichtpakete reagieren und somit nur eine Abschätzung für die Strahlparameter nahe der Strahltaille darstellen können. Für Probe 2 konnte aufgrund des durch die geringe Effizienz schwachen Signals keine Brennweite aus der Strahldivergenz errechnet werden.

Dennoch lässt sich aus den Strahlparametern eines angenommenen Gaußstrahls auch eine erwartete Strahltaille berechnen. Diese wird bei Probe 1 mit $180 \mathrm{~nm}$ bestimmt. Eine experimentelle Methode die Strahlbreite direkt zu vermessen, wird die Schneidblendenrastermessung in Kapitel 3.2.2 liefern. Bei Probe 1 handelt es sich um ein Doppelsystem zweier gegenüber angeordneter identischer Schichtpakete. Die optischen Eigenschaften beider Schichtpakete wurden untersucht. Ein Unterschied der beiden konnte im Experiment nicht beobachtet werden. 


\subsubsection{Schneidblendenrastermessung}

Aufgrund der beobachteten Aberrationen und um die Strahltaille hinter der Multischicht-Laue-Linse direkt zu vermessen, wurde eine Schneidblendenrastermessung (englisch: Moving Knife Edge) durchgeführt. Die Strahlbreite wird dabei nicht direkt, sondern integriert über verschiedene Grade der Strahlabschattung gemessen. Daher wird zunächst eine Methode, die gewonnen Daten bei einem Linienfokus auszuwerten, vorgestellt. Anschließend wird das Experiment erläutert und die Ergebnisse der vermessenen Proben präsentiert.

\section{Bestimmung der Strahlbreite aus der Schneidblendenrastermessung mittels der Methode der zweiten Momente}

Die Strahlbreite des zu vermessenden Strahls soll an verschiedenen Stellen nahe der Strahltaille untersucht werden. Die Messdaten liefern hierbei nicht direkt das Strahlprofil, sondern dessen diskretisierte Stammfunktion. Da die Gesamtzahl an Detektorereignissen bei verschiedenen Strahlabschattungen gemessen wird, bedarf es eines Verfahrens aus diesem integrierten, gegebenenfalls feinstrukturierten Strahlprofil, die Strahlparameter zu bestimmen, denn eine einfache numerische Ableitung der geglätteten Messwerte führt aufgrund der Fehlerfortpflanzung zu wenig robusten Resultaten bei Vergleich verschiedener Messpunkte und -reihen. Mit diesem Verfahren soll direkt die optimal angepasste zweite Momentenbreite des Strahls aus den Messwerten bestimmt werden.

Die räumliche Ausdehnung gerichteter elektromagnetischer Strahlung ist definiert als die Breite senkrecht zur Ausbreitungsrichtung, an der die maximale Intensität auf $1 / e^{2}$ abfällt. Dieses ist auch die relevante Größe bei der Anwendung der Formeln des Gaußstrahls. Allerdings ist diese Definition bei strukturierten Strahlen mit eventuellen Nebenmaxima problematisch, da beispielsweise das Intensitätsniveau mehrfach den $1 / e^{2}$-Abfall durchlaufen kann. Eine einheitliche Methode, die Breite auch von solchen strukturierten Stahlen zu bestimmen, bildet die Methode der Bildmomente [ISO11146, 1999]. Hierbei sind die Bildmomente analog zu den Momenten in der Statistik definiert, sodass das nullte Moment der Gesamtintensität, das erste Moment dem Schwerpunkt des Strahls und das zweite zentrierte Moment seiner Breite entspricht. Im Falle eines Strahlprofils, das der Dichtefunktion der Gaußschen Normalverteilung folgt, entspricht diese Breite gerade dem $1 / e^{2}$-Abfall. Die Strahlbreite $d_{\sigma x}$ eines beliebigen Strahls in $\mathrm{x}$-Richtung wird 
definiert als

$$
\begin{array}{r}
d_{\sigma x}(z)=4 \cdot \sigma_{x}(z) \\
=4 \cdot \sqrt{\frac{\iint(x-\bar{x})^{2} \cdot e(x, y, z) d x d y}{\iint e(x, y, z) d x d y}}, \\
\text { wobei } \bar{x}=\frac{\int x \cdot e(x, y, z) d x}{\int e(x, z) d x} .
\end{array}
$$

Dabei bezeichnet $\bar{x}$ den Schwerpunkt des Strahlprofils und $e(x, y, z)$ die Intensität des Strahls. Analog lässt sich auch die dazu orthogonale Strahlbreite $d_{\sigma y}$ angeben. Im Falle einer eindimensionalen Strahlgeometrie, wie in den folgenden Experimenten und Simulationen beschrieben, vereinfacht sich das Integral um eine Dimension zu

$$
\begin{array}{r}
d_{\sigma}(z)=4 \cdot \sqrt{\frac{\int(x-\bar{x})^{2} \cdot e(x, z) d x}{\int e(x, z) d x}}, \\
\text { wobei } \bar{x}=\frac{\int x \cdot e(x, z) d x}{\int e(x, z) d x} .
\end{array}
$$

Bei einer Strahlbreitenbestimmung mittels der Schneidblendenrastermessung, bei dem der Strahl graduell immer stärker durch eine scharfe Kante abgeschattet wird, misst man die Intensitätsverteilung des Strahls nur indirekt, da lediglich die integrierte Intensitätsverteilung $E(x, z)$ über das eigentliche Strahlprofil $e(x, z)$, also eine Stammfunktion, gemessen werden kann. Das Konzept des Riemann-Stieltjes-Integrals [Schlittgen, 1996, Anhang A], das den Riemann-Integralbegriff verallgemeinert und den Integrator als eine Gewichtungsfunktion auffasst, erlaubt dennoch eine direkte Berechnung der Strahlbreite aus den Messwerten. So kann auf eine numerische Ableitung der diskreten Funktion $E(x, z)$ verzichtet werden. Diese ist aufgrund der wenigen und verrauschten Messwerte weniger robust als dieser direkte Rechenweg. Es gilt:

$$
\begin{aligned}
d_{\sigma}(z)=4 & \sqrt{\frac{\int(x-\bar{x})^{2} \cdot d(E(x, z))}{\int d(E(x, z))}}, \\
& \text { wobei } \bar{x}=\frac{\int x \cdot d(E(x, z))}{\int d(E(x, z))}
\end{aligned}
$$

Die aufgenommenen Daten haben die Struktur einer Treppenfunktion, da sie nur an diskreten Messpunkten erhoben werden können, somit gilt

$$
E(x)=\sum_{i=1}^{s} h_{i} \cdot \mathbb{1}_{\left\{x_{i} \leq x\right\}},
$$




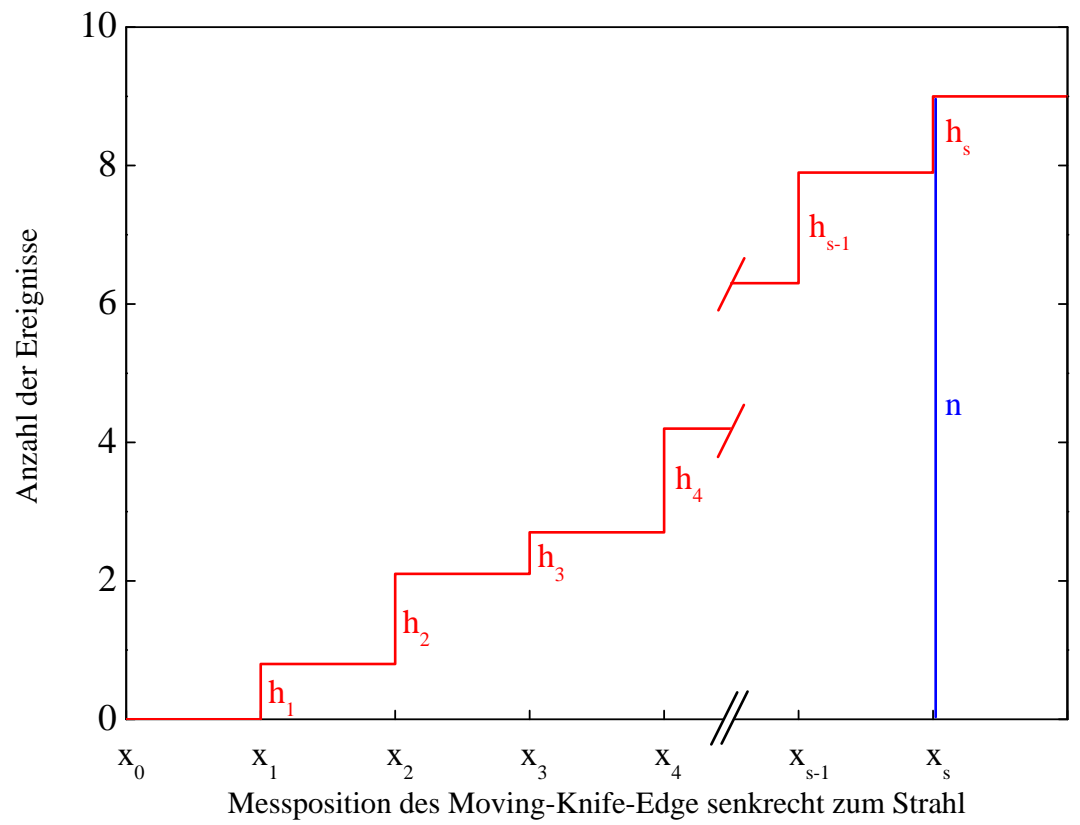

Abbildung 3.7:

Grafische Darstellung der zur Modellierung der Messwerte der Schneidblendenrastermessung verwendeten Treppenfunktion. Die Differenz der zwischen zwei Messpositionen $x_{i}$ und $x_{i+1}$ aufgenommenen Daten ist die Sprunghöhe $h_{i}$. Die Zahl der Stufen ist $s, n=\sum h_{i}$ die absolute Höhendifferenz, also die Gesamtintensität des Strahls.

wobei $x_{i}$ mit $i=1, \ldots, s$ die Sprungstellen dieser Treppenfunktion und damit die verschiedenen Positionen der Schneidblendenrastermessung beschreibt. $h_{i}$ ist dann die Sprunghöhe an einer solchen Position und $\mathbb{1}_{\left\{x_{i} \leq x\right\}}$ die Indikatorfunktion, die für alle $x_{i}<x$ gleich 0 ist und 1 sonst. Abbildung 3.7 illustriert die verwendeten Größen. Es gilt somit für das nullte Bildmoment, welches der detektierten Gesamtintensität entspricht:

$$
\int d E(x)=\int d\left(\sum_{i=1}^{s} h_{i} \cdot \mathbb{1}_{\left\{x_{i} \leq x\right\}}\right) .
$$

Aus der Linearität des Stielties-Integrals im Integrator $\left(\int f(x) \cdot d(\alpha g(x)+\beta h(x))=\right.$ $\left.\alpha \int f(x) \cdot d g(x)+\beta \int f(x) \cdot d h(x)\right)$ [Schlittgen, 1996] folgt

$$
\begin{aligned}
\int d E(x) & =\sum_{i=1}^{s} h_{i} \\
& =n,
\end{aligned}
$$

wobei $n$ der Gesamtzahl der registrierten Detektorereignisse bei nicht abgeschattetem 
Strahl entspricht. Für das erste Bildmoment gilt dann:

$$
\begin{aligned}
\int x d E(x) & =\int x d\left(\sum_{i=1}^{s} h_{i} \cdot \mathbb{1}_{\left\{x_{i} \leq x\right\}}\right) \\
& =\sum_{i=1}^{s} x_{i} \cdot h_{i} .
\end{aligned}
$$

Eine intuitivere Interpretation dieses Ergebnisses, welches der Schätzung des Erwartungswertes durch den Mittelwert entspricht, gewinnt man durch eine andere Darstellung der Summe über die Detektorereignisse an einer Messpositionen. Diese kann als die Summe aller einzelnen Detektorereignisse an einer Messposition ausgedrückt werden.

$$
\begin{aligned}
\int x d E(x) & =\sum_{i=1}^{s} \sum_{j=1}^{h_{i}} x_{i} \\
& =\sum_{k=1}^{n} x_{k}
\end{aligned}
$$

Daraus folgt zusammen mit dem nullten Bildmoment in Gleichung (3.18):

$$
\begin{aligned}
\bar{x} & =\frac{\int x d E(x)}{\int d E(x)} \\
& =\frac{1}{n} \sum_{k=1}^{n} x_{k} .
\end{aligned}
$$

$\bar{x}$ ist somit das erste normierte Bildmoment und gibt den Strahlschwerpunkt an. Analog lässt sich auch das zweite Bildmoment darstellen:

$$
\begin{aligned}
\int(x-\bar{x})^{2} d E(x) & =\int(x-\bar{x})^{2} \cdot d\left(\sum_{i}^{s} h_{i} \cdot \mathbb{1}_{\left\{x_{i} \leq x\right\}}\right) \\
& =\sum_{i=1}^{s}\left(x_{i}-\bar{x}\right)^{2} h_{i} \\
& =\frac{1}{n} \sum_{k=1}^{n}\left(x_{k}-\bar{x}\right)^{2} .
\end{aligned}
$$

Das zweite zentrierte und normierte Bildmoment und damit die Breite des Strahls ergibt sich dann durch den Quotienten der Terme aus Gleichung (3.23) und Gleichung (3.18) sowie dem Strahlschwerpunkt aus Gleichung (3.22). Sie lässt sich anschaulich als 


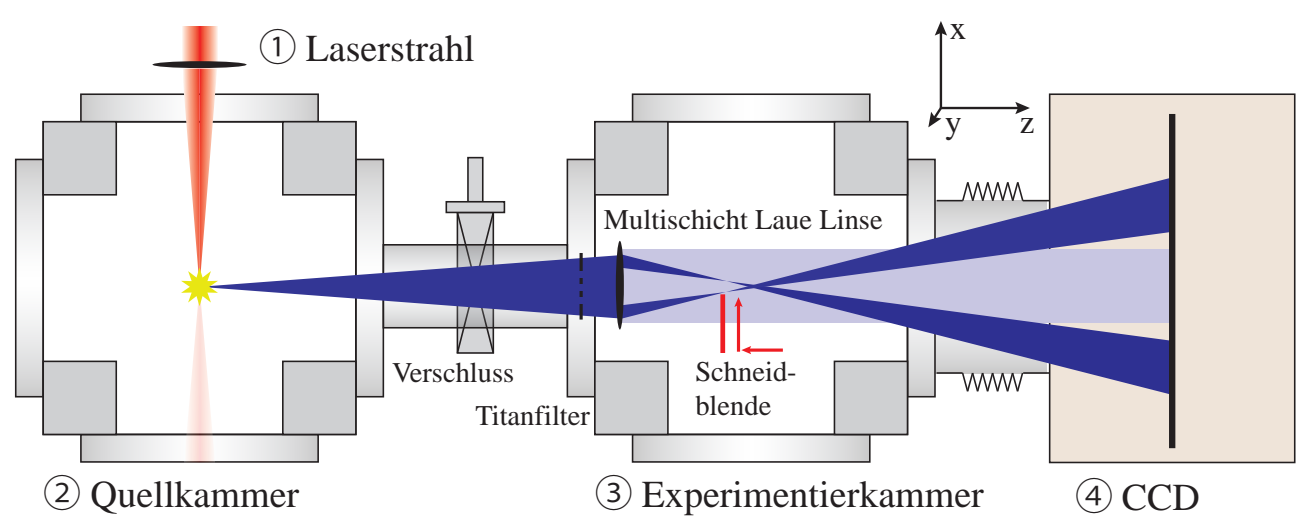

Abbildung 3.8:

Aufbau des Schneidblenden-Experiments. Der Aufbau ist modular in verschiedenen Kammern gestaltet. Das Plasma wird durch den Laser (1) in der Quellkammer (2) gezündet. Die entstandene XUV-Strahlung fällt in einigem Abstand über ein System von Aperturen und Blenden, gefiltert durch eine Titanfolie, auf die Probe in der Experimentierkammer (3). In der Experimentierkammer befindet sich auch die Schneidblende, die mit zwei Piezomotoren an die Messposition gefahren wird. Eine CCD-Kamera (4) unter fixem Abstand detektiert das Beugungsbild hinter der Probe.

Standardabweichung der Strahlintensitätsverteilung beschreiben.

$$
\begin{aligned}
d_{\sigma} & =4 \sqrt{\frac{\sum_{k=1}^{n}\left(x_{k}-\bar{x}\right)^{2}}{\sum_{k=1}^{n} 1}} \\
& =4 \sqrt{\frac{\sum_{k=1}^{n}\left(x_{k}-\bar{x}\right)^{2}}{n}}
\end{aligned}
$$

\section{Experiment}

Das Experiment zur Bestimmung der Strahlparameter nahe der Strahltaille ist schematisch in Abbildung 3.8 illustriert. Der Aufbau ähnelt in wesentlichen Punkten dem Divergenzwinkelexperiment, das in Kapitel 3.2.1 beschrieben wird. Wegen der hohen Absorption der extrem ultravioletten Strahlung muss der Versuch unter Vakuum stattfinden. Dazu wurde in einem gasförmigen Stickstofftarget mit einem Laser (Innolas Spitlight 600, ausgewählte Parameter in Tabelle 2.1 auf Seite 26) ein Plasma gezündet. Aus der schmalbandigen Abstrahlcharakteristik in Kombination mit Filterung durch eine Titanfilterfolie resultiert eine monochromatische Beleuchtung bei 2,88 nm Wellenlänge.

Hinter einem System von Aperturen befindet sich in einem Abstand von $83 \mathrm{~cm}$ das Schichtpaket. Direkt hinter diesem ist ein gespannter Metalldraht von $10 \mu \mathrm{m}$ Durchmesser montiert. Dieser wird zu dem Linienfokus der Multischicht-Laue-Linse erst grob mit einem Mikroskopobjektiv mit langem Arbeitsabstand (Questar QM100) ausgerich- 


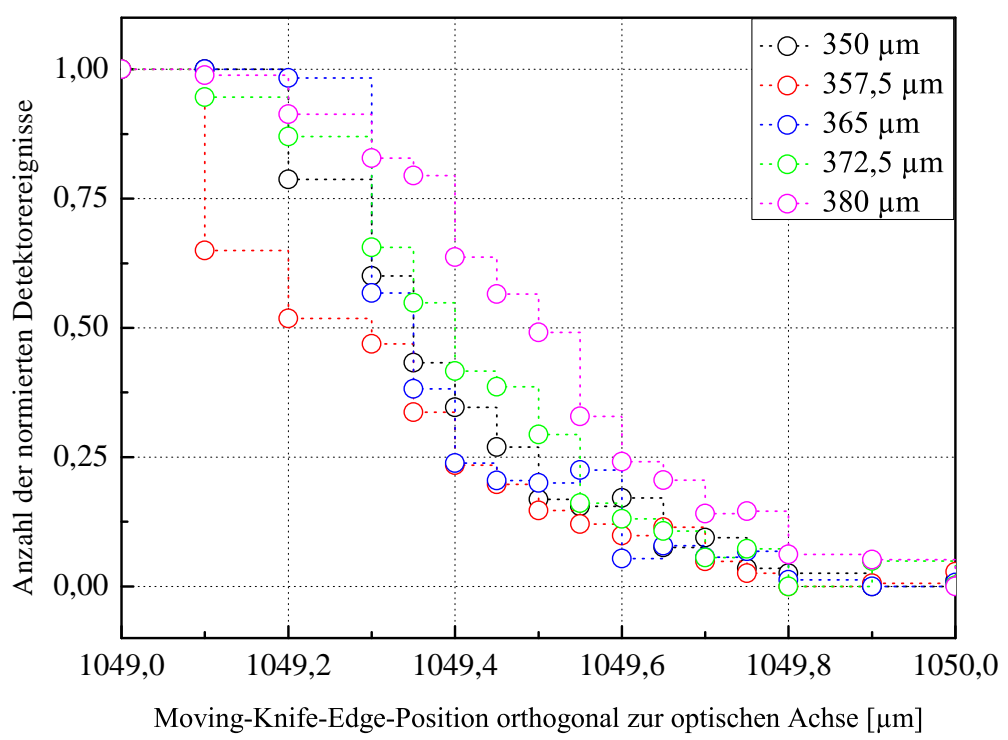

Abbildung 3.9:

Multischicht-Laue-Linse 1b: Auszug von den in der Schneidblendenrastermessung gewonnenen Rohdaten. Entlang der optischen Achse wurde an verschiedenen Positionen die Strahlbreite durch zunehmende Abschattung der auf dem CCD Detektor gemessenen extrem ultravioletten Strahlung $(2,88 \mathrm{~nm})$ bestimmt. Die Messreihen wurden jeweils bei maximaler Intensität gestartet, bis zu einer vollständigen Abschattung des Strahls durchgeführt und sind auf die maximale Intensität normiert [Reese u. a., 2011].

tet. Später kann diese Vorjustage mit Resten von durch die Aperturblende tretender XUV-Strahlung genau korrigiert werden. Der Draht kann durch zwei Piezomotoren (Mechonics PS30CL, $8 \mathrm{~mm}$ Verfahrweg, $50 \mathrm{~nm}$ Positioniergenauigkeit) längs und senkrecht zur optischen Achse bewegt werden. Das Bild auf dem CCD-Detektor (Roper Scientific PI-SX:1k, $1024^{2}$ Pixel, 13x13 $\mu \mathrm{m}^{2}$ Pixelgröße) entspricht dem des Divergenzwinkelexperiments bei einem fixierten Detektorabstand (siehe Abbildung 3.4 auf Seite 59). Durch Integration der Detektorereignisse über 1200 Pulse bei $10 \mathrm{~Hz}$ Repetitionsrate wird ein gut ausgesteuertes Signal auf dem Detektor erreicht. Ein Einzelpuls entspricht dabei etwa 2400 Photonen auf der Apertur der Multischicht-Laue-Linse. Durch schrittweises Abschatten des Strahls durch den Draht als Schneidblende entlang verschiedener Positionen der optischen Achse kann die Strahltaille gefunden und vermessen werden.

Im Experiment zeigte sich, dass die Herstellung der Multischicht-Laue-Linse 1a/b fehlerbehaftet war. Da beide Schichtpakete keinen gemeinsamen Bildpunkt erzeugen, ist für die weitere Analyse nur die fokussierende Beugungsordnung eines Schichtpakets berücksichtigt. Der Strahl hinter Probe 1b wurde wie beschrieben vermessen. Ein Teil der Messkurven eines solchen Experiments ist in Abbildung 3.9 aufgetragen. Ein Unterschied der optischen Eigenschaften der beiden einzelnen Schichtpakete konnte nicht festgestellt werden. 
Der Nullpunkt der Messung ist experimentell bedingt und kein absoluter Abstand zur Probe. Jeder dieser Messreihen lässt sich nach der Momentenmethode aus Kapitel 3.2.2 eine Strahlbreite zuordnen. In Abbildung 3.10 sind diese Breiten gegen die Position der Schneidblende entlang der optischen Achse aufgetragen. Die Strahltaille zeigt dabei annähernd einen Verlauf, wie er für einen Gaußstrahl charakteristisch wäre. Es lässt sich deshalb, der Mathematik eines Gaußstrahls folgend, eine hyperbolische Anpassung an die Strahltaille vornehmen. Aus dieser Anpassung lassen sich alle relevanten Gaußstrahlparameter ableiten.

Die Messung des Strahlprofils, die der Strahltaille am nächsten ist, ist in Abbildung 3.11 aufgetragen. Neben den Messdaten ist auch die Normalverteilungsfunktion mit den aus der Momentenmethode gewonnenen Parametern dargestellt, die dem zugehörigen Profil eines Gaußstrahls entsprechen (siehe Anhang B). Zu dieser korrespondiert eine Dichteverteilung, die dem zugehörigen Strahlprofil entspricht. Es ergibt sich eine Strahlbreite des entsprechenden Gaußstrahls an der Strahltaille von $375 \pm 30 \mathrm{~nm}$ Halbwertsbreite.

Die experimentell bestimmten Strahlparameter hinter der Multischicht-Laue-Linse, wie in Reese u. a. [2011] veröffentlicht, werden in Tabelle 3.3 zusammenfassend angegeben. Da der Divergenzwinkel im Divergenzwinkelexperiment mit höherer Genauigkeit bestimmt wurde, wird der dort gewonnene Wert gewählt und in die Brechnungen einbezogen.

Weil Probe 2 ein zu geringes Signal in der ersten Beugungsordnung liefert, konnte hier keine Messung mit der Schneidblende durchgeführt werden. Die bestimmbaren Strahlparameter beschränken sich daher auf den Beugungswinkel aus dem Divergenzwinkelexperiment.

Tabelle 3.3:

Eigenschaften des Strahls hinter der Multischicht-Laue-Linse 1b, bestimmt aus einer Kombination der Schneidblendenrastermessung und des Divergenzwinkelexperiments. [Reese u. a., 2011]

\begin{tabular}{lc}
\hline $\begin{array}{l}\text { Divergenzwinkel } \\
\text { (Divergenzwinkelexperiment) }\end{array}$ & $20,4 \pm 0,3 \mathrm{mrad}$ \\
$\begin{array}{l}\text { Halbwertsbreite der Strahltaille } \\
\text { (Schneidblendenrastermessung) }\end{array}$ & $375 \pm 30 \mathrm{~nm}$ \\
Rayleighlänge & $31,3 \pm 2,5 \mu \mathrm{m}$ \\
Brennweite & $205 \pm 24 \mu \mathrm{m}$
\end{tabular}




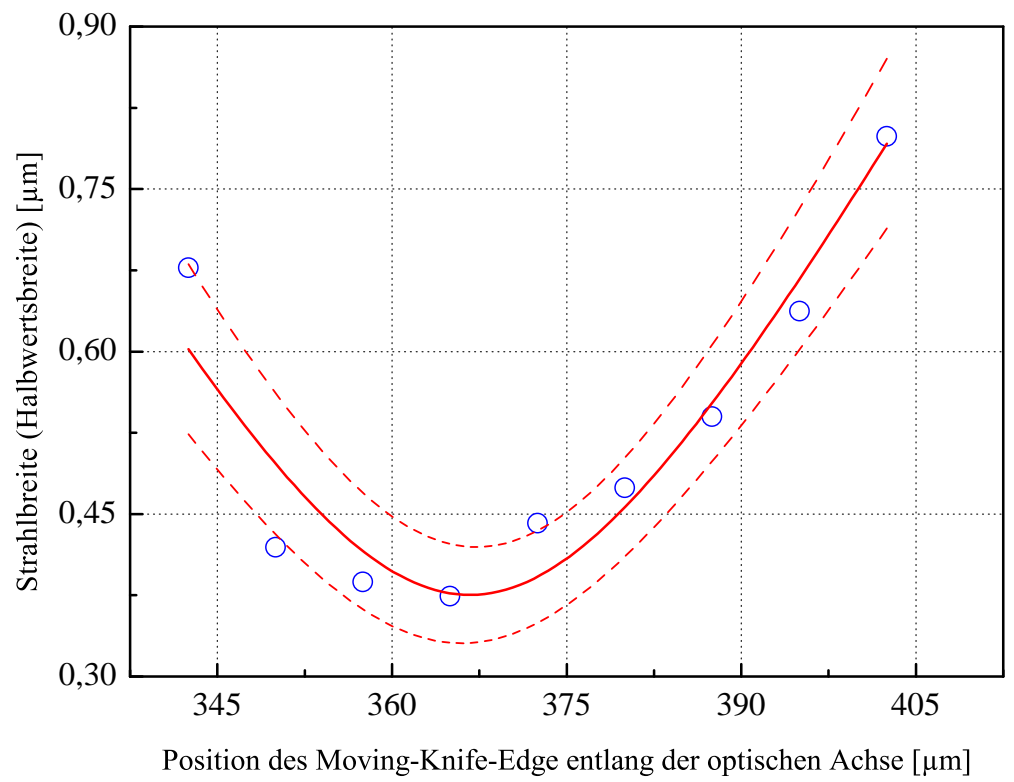

Abbildung 3.10:

Multischicht-Laue-Linse 1b: Die aus den Einzelmessreihen bestimmten Strahlbreiten. Das Profil der Strahltaille ist vergleichbar mit dem eines Gaußstrahls. Daher wurde eine hyperbolische Anpassung der Strahltaille mit $95 \%$-Konfidenzbändern (Origin 8.5) aufgetragen [Reese u. a., 2011].

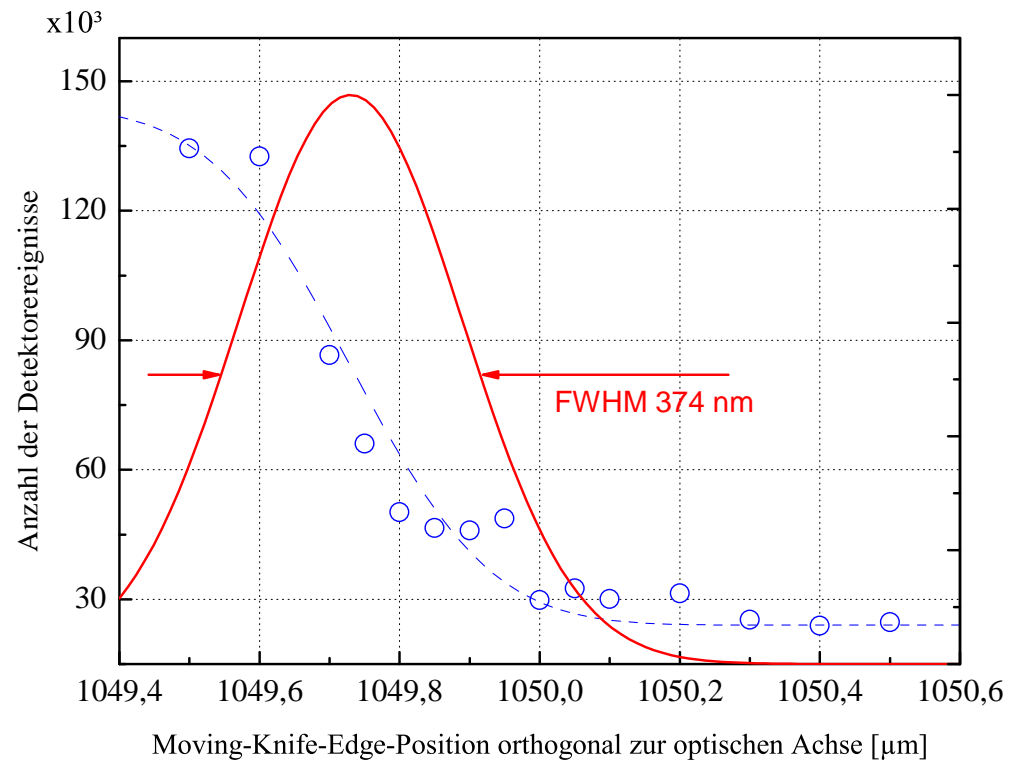

Abbildung 3.11:

Multischicht-Laue-Linse 1b: Auftragung des Strahlprofils, das am nächsten der Strahltaille gelegen ist. Die Normalverteilung wurde mit den aus der zweiten Momentenmethode gewonnen Parametern dargestellt. Die zugehörige Dichtefunktion dieser Normalverteilung repräsentiert das Strahlprofil eines angenommenen Gaußstrahls, seine Halbwertsbreite ist mit $374 \pm 30 \mathrm{~nm}$ bestimmt [Reese u. a., 2011]. 


\subsubsection{Messung am Synchrotron}

Um die aus dem Laborexperiment gewonnenen Daten zu verifizieren, wurden an der Synchrotronstrahlquelle BESSY II in Berlin durch Dong-Du Mai in Kooperation mit Prof. Dr. Hans-Ulrich Krebs und Tobias Liese sowie der Arbeitgruppe Dr. Axel Rosenhahn der Universität Heidelberg durchgeführte Messungen (August 2010) zur Bestimmung des Beugungs- und Divergenzwinkels ausgewertet. Das Experiment war dabei weitgehend identisch mit dem an der Laborstrahlungsquelle durchgeführten Divergenzwinkelexperiment, wobei die inkohärente Plasmaquelle durch den kohärenten Synchrotronstrahl bei einer Wellenlänge $\lambda$ von $2,88 \mathrm{~nm}$ ersetzt wurde.

Genutzt wurde dazu der Ptychographie-Versuchsaufbau HORST der Universität Heidelberg [Mancuso u.a., 2010; Beckers u.a., 2011]. Die Multischicht-Laue-Linse wurde hinter einer Lochblende in den Synchrotronstrahl gestellt und das entstehende Beugungsbild mit einer rückseitig gedünnten CCD-Kamera aufgenommen (Andor Technology, 2048 ${ }^{2}$ Pixel, 13, $5^{2} \mu \mathrm{m}^{2}$ Pixelgröße). Eines der detektierten Bilder ist beispielhaft in Abbildung 3.12 gezeigt. Wie bereits in Kapitel 3.2.1 für die Laborstrahlungsquelle

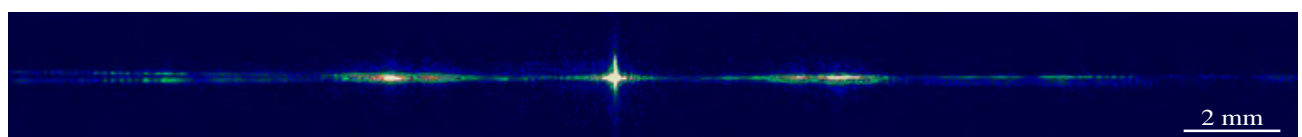

Abbildung 3.12:

Beispiel der Aufnahmen am BESSY II. Die Ähnlichkeit zu den Experimenten an der Laborstrahlungsquelle (Abbildung 3.4) ist klar zu erkennen. Eine genaue Position hinter der CCD-Kamera ist nicht bekannt, kann aber aus den voraussichtlichen Schnittpunkten von 1. und 0 . Beugungsordnung mit $14 \mathrm{~cm}$ abgeschätzt werden. Das Auftauchen einer 2. Beugungsordnung deutet auf Aberrationen hin, die durch nicht optimale Zonenabstände oder die endliche Dicke [Kurokhtin und Popov, 2002] hervorgerufen sein könnten.

durchgeführt, lässt sich aus den Momentenbreiten des Strahls ein Divergenzwinkel aus der linearen Regression der Auftragung der Strahlbreiten über den relativen Detekto-

Tabelle 3.4:

Der Beugungswinkel $\beta$ bestimmt aus dem Divergenzwinkelexperiment und deren Vergleich mit den Simulationsergebnissen. Es findet sich insgesamt eine gute Übereinstimmung. Bei Probe $1 \mathrm{a} / \mathrm{b}$ sind jeweils auch die Divergenzwinkel aus den Simulationen der mit linearen Schichtdickenfehler behafteten Multischicht-LaueLinse angegeben.

\begin{tabular}{|c|c|c|c|c|}
\hline & $\begin{array}{l}\text { Beugungswinkel } \\
\text { aus Simulation }\end{array}$ & $\begin{array}{l}\text { Beugungswinkel } \beta \\
\text { Plasmaquelle }\end{array}$ & $\begin{array}{l}\text { Beugungswinkel } \beta \\
\text { BESSY II }\end{array}$ & Divergenzwinkel $\theta$ \\
\hline MLL Probe 1a/b & $\begin{array}{l}1,61^{\circ} \\
\left(1,80^{\circ} \quad \text { mit }\right. \\
\text { Fehler })\end{array}$ & $1,83^{\circ}$ & $1,76^{\circ}$ & $20,4 \pm 0,3 \mathrm{mrad}$ \\
\hline MLL Probe 2 & $7,78^{\circ}$ & $8,29^{\circ}$ & & \\
\hline
\end{tabular}




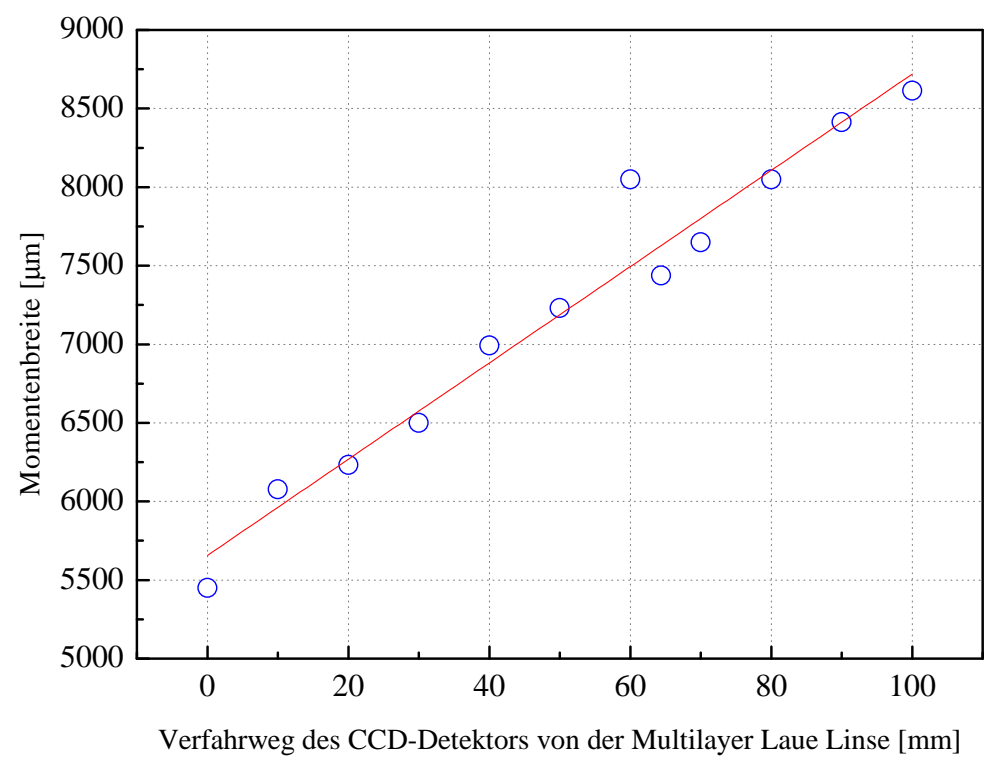

Abbildung 3.13:

Probe 1b: Bestimmung des Divergenzwinkels $\theta$ aus den Strahlbreiten eines Schichtpakets gemessen am BESSY II. Die lineare Regression ergibt eine Strahldivergenz von 30,5 mrad.

rabstand bestimmen. Diese ist in Abbildung 3.13 gezeigt.

Der Divergenzwinkel wird mit 30,6 $\pm 2,1 \mathrm{mrad}$ bestimmt. In Tabelle 3.4 befindet sich eine Zusammenstellung der verschiedenen bestimmten Winkel. Unter der Annahme eines Gaußstrahls, trotz der Beobachtung von starken Aberrationen, kann eine minimale Strahltaillenbreite von $111 \mathrm{~nm}$ errechnet werden. Wie schon an der Laborquelle beobachtet, zeigt sich eine ausgeprägte Feinstrukturierung des 1. Beugungsmaximums. Da diese qualitativ identisch auch bei der hochmonochromatischen Synchrotronstrahlung beobachtet wird, kann ausgeschlossen werden, dass es sich um einen Effekt der Laborquelle handelt. Ein direkter Vergleich findet sich in Abbildung 3.14. Auch das Erscheinen der zweiten Beugungsordnung im Detektorbild deutet auf Aberrationen durch ungleichmäßig strukturierte Schichten und die endliche Dicke der Multischicht-Laue-Linse hin, da diese bei einer optimalen Zonenplatte unterdrückt sind. 


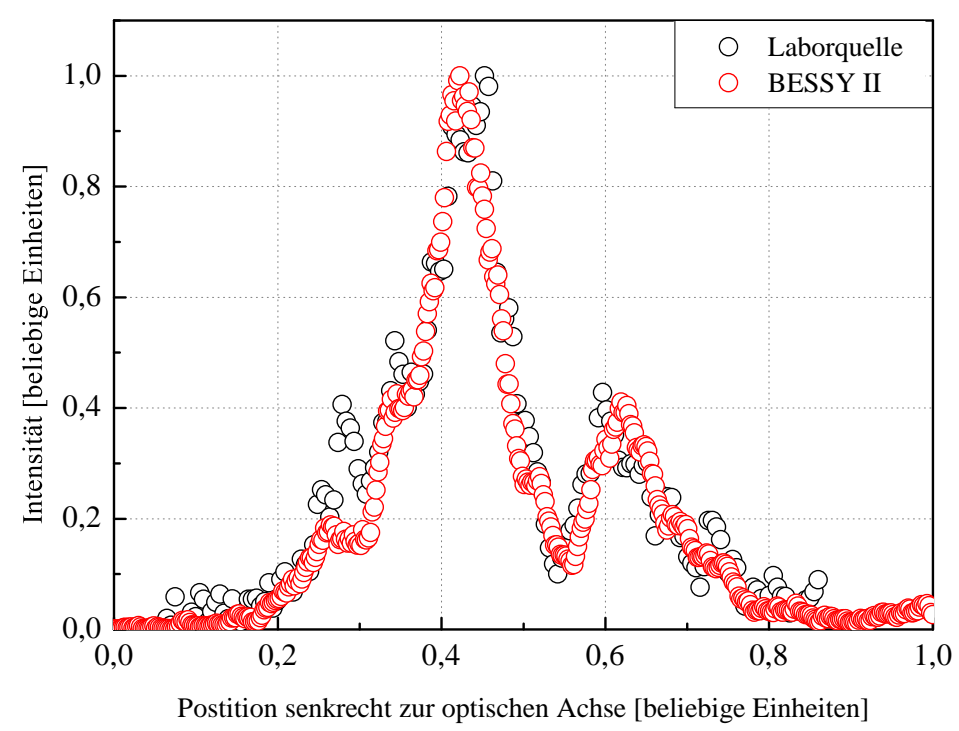

Abbildung 3.14:

Probe 1b: Die starke Feinstrukturierung des 1. Beugungsmaximums wird bei Labor- wie Synchrotronquelle beobachtet. Da die Messabstände der Probe zum CCD-Detektor der jeweiligen Messungen nicht identisch sind, wurden die Einheiten beliebig gewählt, um die Ähnlichkeit der Feinstrukturierung zu betonen. Der CCD-Detektor befindet sich in der Labormessung bei den aufgetragenen Strahlprofilen $80 \mathrm{~mm}$ hinter der Multischicht-Laue-Linse in der Messung am BESSY II etwa $140 \mathrm{~mm}$. 


\subsubsection{Farbzentrenerzeugung}

In diesem Kapitel wird ein kurzer Überblick über eine Methode zur Strahlcharakterisierung mit Hilfe der Erzeugung von Farbzentren gegeben. Farbzentren in Lithium-FluoridKristallen wurden bereits erfolgreich zur Charakterisierung des Strahls von Röntgenlasern [Tomassetti u. a., 2003] und als Detektor zur Röntgenmikroskopie an Laborquellen eingesetzt [Reale u. a., 2008].

Durch Bestrahlung von geeigneten nichtleitenden Ionenkristallen mit hochenergetischen XUV-Photonen lassen sich Fehlstellen im Ionengitter erzeugen [Lammel, 2003]. Als Farbzentren bezeichnet man das Ersetzen eines oder mehrerer fehlender Anionen durch ein oder mehrere Elektronen. Diese Elektronen können sichtbares Licht absorbieren und zur Fluoreszenz angeregt werden. Zur Erhöhung der räumlichen Auflösung bei mikroskopischer Analyse der belichteten Flächen kann stimulierte Emissions-Löschung ausgenutzt werden (STED englisch: stimulated emission depletion). „Das STED-Mikroskop arbeitet mit zwei synchronisierten Laserpulsen: ein ,blauer' Laserpuls regt die Moleküle zur Fluoreszenz an, ein kurz darauf folgender ,roter ${ }^{k}$ Puls mit einem Minimum in der Mitte des Brennflecks löscht die Fluoreszenz am Randbereich des Fokus wieder ab. Je intensiver der Löschpuls, desto kleiner wird der übrig gebliebene Fluoreszenzfleck, sodass der Brennfleck theoretisch - trotz der Beugung des Lichts - bis auf Molekülgröße verkleinert werden kann. Damit konnte Hell ein physikalisch schlüssiges Verfahren angeben, mit dem die Auflösung von der Wellenlänge entkoppelt werden konnte.“[Hell, 2003] Der für die Messung verwendete Mikroskopieaufbau ist bei Pezzagna u. a. [2010] beschrieben.

Ziel ist es, durch Belichtung mit kleinsten Strahltaillen von wenigen $10 \mathrm{~nm}$ und die anschließende Analyse der erzeugten Farbzentren mit STED-Mikroskopie die Kaustik um den Fokus in einer, verglichen mit den Schneidblendenrastermessungen, kurzen Zeit mit hoher räumlicher Auflösung zu detektieren.

Um die Eignung von LiF-Kristallen hierzu zu überprüfen, wurde ein Kristall (Korth Kristalle $10 \mathrm{~mm} \varnothing, 1 \mathrm{~mm}$ Dicke) in $40 \mathrm{~cm}$ Abstand zu einer Laserplasmaquelle (Stickstoffgastarget, $200 \mathrm{~nm}$ Ti-Filter, $5 \mathrm{~Hz}$ Repetitionsrate, Quantel YG980), in allen drei Raumrichtungen verfahrbar (Mechonics PS30CL, $8 \mathrm{~mm}$ Hub, $50 \mathrm{~nm}$ Positioniergenauigkeit), hinter einer Lochblende $(50 \mu \mathrm{m} \varnothing)$ montiert. Ein direkt aufliegendes TEM-Gitter (4,5 $\mu \mathrm{m}$ Stegbreite) soll eine klar definierte Struktur im beleuchteten Bereich mit scharfen Kanten erzeugen.

Die Belichtung mit XUV-Strahlung über 20, 10 und 2 Minuten erzeugt fluoreszente Farbzentrenbereiche auf dem Kristall, die bereits mit einem Fluoreszenzmikroskop (Leica DM6000, RGB-Filter, Abbildung 3.15(a)) detektierbar sind. Die im LiF-Kristall erzeugbaren Farbzentren mit ihren Absorptions- und Emissionsenergien sind in Abbildung 3.15(b) dargestellt.

Die beleuchteten Bereiche wurden in einem STED-Mikroskop (Anregungswellenlänge 


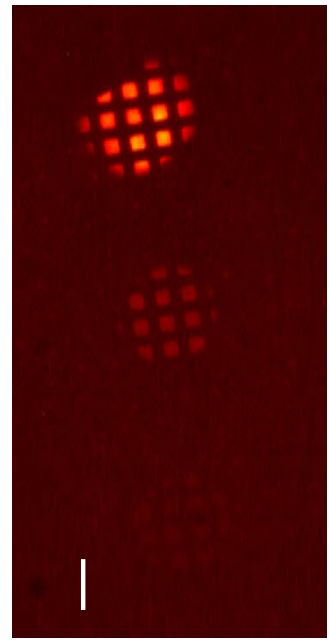

(a) Fluoreszenzmikroskopaufnahme, 20× Vergrößerung, RGB-Filter, der Balken entspricht $20 \mu \mathrm{m}$.

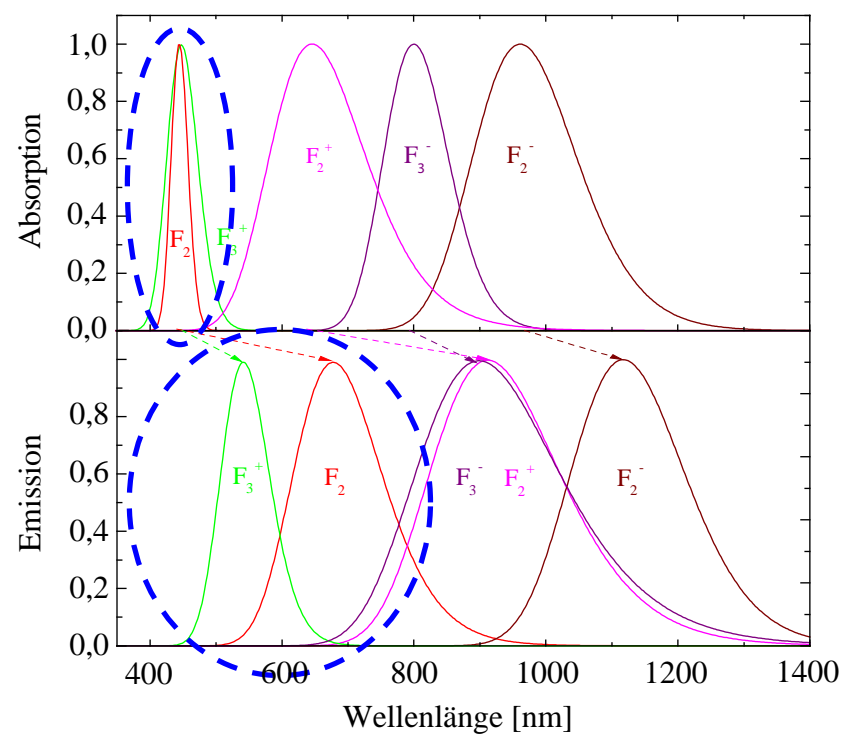

(b) Anregungs- und Abregungsenergien für verschiedene LiFFarbzentren [Bonfigli u.a., 2004]. Die Zahl indiziert die im Farbzentrum fehlenden Anionen und das Vorzeichen die relative Zahl ersetzender Elektronen. Die im Fluoreszenz- und STEDMikroskop untersuchten Farbzentren sind mit einem blauen Kreis gekennzeichnet.

\section{Abbildung 3.15:}

Mittels monochromatischer XUV-Strahlung von 2,88 nm Wellenlänge werden in einem LiF-Kristall Farbzentren erzeugt. Die Belichtungen hinter einer Lochblende $(\varnothing 50 \mu \mathrm{m})$ und einem TEM-Gitter $(4,5 \mu \mathrm{m}$ Stegbreite) wurden über 20, 10 und 2 Minuten ((b) - von oben nach unten) durchgeführt. Die entstandenen Farbzentren fluoreszieren im sichtbaren Spektralbereich.

$430 \mathrm{~nm}$, STED-Wellenlänge $755 \mathrm{~nm}$ ) auf ihre Eignung für das STED-Verfahren untersucht. Dazu wurde zunächst ein Fluoreszenzbild (Abbildung 3.16(a)) aufgenommen und anschließend eines nach Hinzuschaltung des STED-Lasers (Abbildung 3.16(b)). Die Größe der belichteten Struktur liegt nicht in dem Bereich, in dem eine Auflösungserhöhung durch die STED-Methode zu erwarten ist, sodass lediglich die detektierte Intensität durch die stimulierte Abregung der Farbzentren geringer wird. Es zeigt sich ein Bleichen der Farbzentren bei der Exposition mit dem starken Laserlicht des Anregungsund STED-Lasers. Bei erneuter konfokaler Betrachtung der untersuchten Stelle zeigt sich daher ein deutlicher Signal- und Kontrastverlust (Abbildung 3.16(c)).

Um ein genaueres Bild von der Anwendbarkeit der Methode zu gewinnen, wird eine sogenannte Leerungskurve (englisch: depletion curve) aufgenommen, um die Effektivität der STED-Methode quantitativ abschätzen zu können. Dazu wird das Fluoreszenzsignal erzeugt durch den Anregungslaser, durch den Anregungslaser zusammen mit dem 


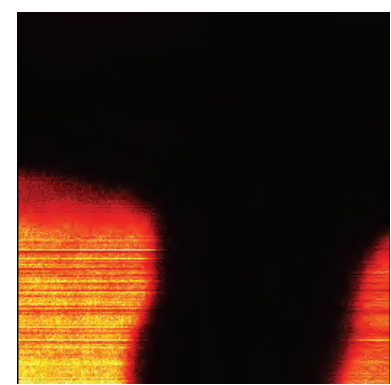

(a) Fluoreszenzmikroskopische Aufnahme (Bildbereich $\left.10 \times 10 \mu \mathrm{m}^{2}\right)$.

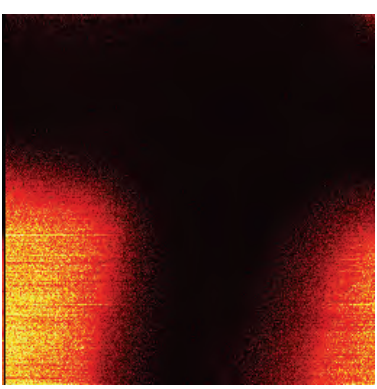

(b) STED Fluoreszenzmikroskopische Aufnahme (Bildbereich $10 \times 10 \mu \mathrm{m}^{2}$ ).

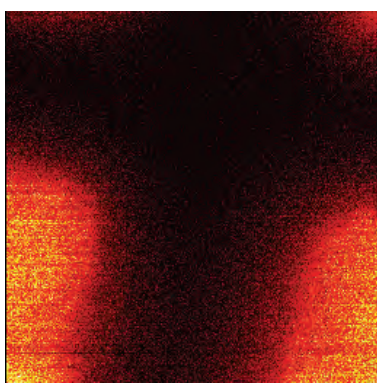

(c) Fluoreszenzmikroskopische Aufnahme (Bildbereich $\left.10 \times 10 \mu \mathrm{m}^{2}\right)$.

\section{Abbildung 3.16:}

STED-Mikroskopie der LiF-Farbzentren. Der belichtete Kristall wird im STED-Mikroskop untersucht, wobei (a) - (c) nacheinander an gleichet Stelle aufgenommen wurden. Aufgetragen sind die relativen Intensitäten zum Maximum des jeweiligen Bildes. Die Probe ist zwar mit der Methode untersuchbar, allerdings zeigt sich ein starkes Bleichen der Farbzentren. Die vertikalen Streifen rühren von einem Flackern des Anregungslasers her.

STED-Laser und durch den STED-Laser alleine aufgenommen. Diese Messung wurde für steigende Leistungen des STED-Lasers wiederholt. In Abbildung 3.17(a) sind Fluoreszenzsignal des Anregungslasers und Fluoreszenzsignal bei Bestrahlung mit Anregungslaser und STED-Laser abzüglich des Signals bei Beleuchtung nur mit dem STED-Laser dargestellt. Aus der relativen Fluoreszenz zueinander ergibt sich eine Senkung des Fluoreszenzsignals um $75 \%$ bei maximaler STED-Intensität (Abbildung 3.17(b)). Dieser Wert ist, verglichen mit anderen mit dieser Methode untersuchten Materialsystemen, nicht sehr hoch. Dennoch kann eine höhere räumliche Auflösung als mit konfokaler Mikroskopie erwartet werden.

Diese Methode ist nicht auf die Charakterisierung von Röntgenoptiken beschränkt. Lithiumfluorid oder ein anderer geeigneter Kristall als Detektor und die STED-Mikroskopie als Analysemethode könnten mikroskopische Untersuchungen an Laborquellen mit Auflösungen unter $100 \mathrm{~nm}$ möglich machen.

\subsection{Simulationen}

Um ein tieferes Verständnis der Strahlpropagation und um genauere Aussagen über die zu erwartende Abbildungsleistung und die Strahlkaustik zu erhalten, entstand im Rahmen dieser Arbeit ein Programm zur Simulation des Experiments in Matlab ( [MathWorks Inc., 2009]. Zunächst wird dazu der Programmcode (siehe Anhang C) erläutert und anschließend die Ergebnisse der Simulation diskutiert. Hierbei wird gezeigt, dass das erreichbare Auflösungsvermögen entscheidend von der Zahl der Zonen, also der 


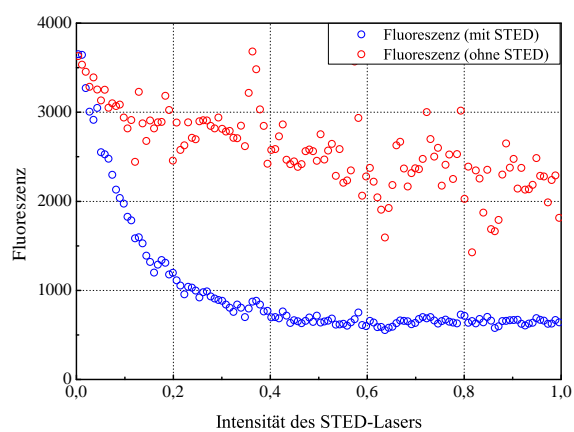

(a) Untersuchung des Fluoreszenz-Signals als Funktion der relativen Intensität des STEDLasers.

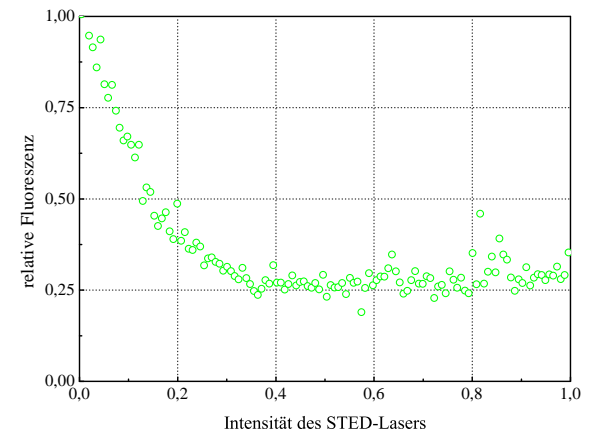

(b) relative Fluoreszenz der Anregung mit und ohne STED-Laser.

Abbildung 3.17:

Untersuchung des Fluoreszenz-Signals als Funktion der relativen Intensität des STED-Lasers. Die Abnahme des Signals bei steigender STED-Energie bestätigt die erfolgreiche Abregung des Signals durch den STEDLaser.

numerischen Apertur der diffraktiven Optik, abhängt. Die Simulationen geben sowohl die optimalen Eigenschaften der hergestellten Proben als auch modellierte Herstellungsund Experimentierfehler wieder.

\subsubsection{Das Kirchhoffsche Beugungsintegral}

Die Ausbreitungseigenschaften der elektromagnetischen Wellen der Plasmaquelle und deren Interaktion mit der Multischicht-Laue-Linse werden im Folgenden beschrieben. Die Propagation einer elekromagnetischen Welle kann durch das Kirchhoffsche Beugungsintegral vollständig beschrieben werden. Dazu wird an einem beliebigen Punkt im Raum das komplexe Feld aller infinitesimal kleinen Beleuchtungspunkte aufaddiert. Diese Vorgehensweise ist als das Huygens'sche Elementarwellen-Prinzip bekannt und wird in Abbildung 3.18 illustriert. Die von links einstrahlende, vollständig beschriebene Welle wird durch eine ebenfalls bekannte Transmissionsfunktion $\tau\left(x_{1}, y_{1}, 0\right)$ moduliert. Ausgehend von den entstehenden Elementarwellen an den Punkten $\left(x_{0}, y_{0}, 0\right)$ kann über den Abstandsvektor $\overrightarrow{\mathbf{r}}$ auf jedem Punkt $\left(x_{1}, y_{1}, z\right)$ einer Ebene $E\left(x_{1}, y_{1}, z\right)$ das resultierende Feld durch die festen Phasenbeziehungen berechnet werden. Es ergibt sich das Kirchhoffsche Beugungsintegral, wie es beispielsweise bei Lauterborn und Kurz [2003, Kapitel 9.1] beschrieben ist:

$$
E\left(x_{1}, y_{1}, z\right)=\frac{1}{i \lambda} \iint_{-\infty}^{\infty} E\left(x_{0}, y_{0} 0\right) \frac{e^{i k|\overrightarrow{\mathbf{r}}|}}{|\overrightarrow{\mathbf{r}}|} \cos (\overrightarrow{\mathbf{n}} \overrightarrow{\mathbf{r}}) d x d y
$$




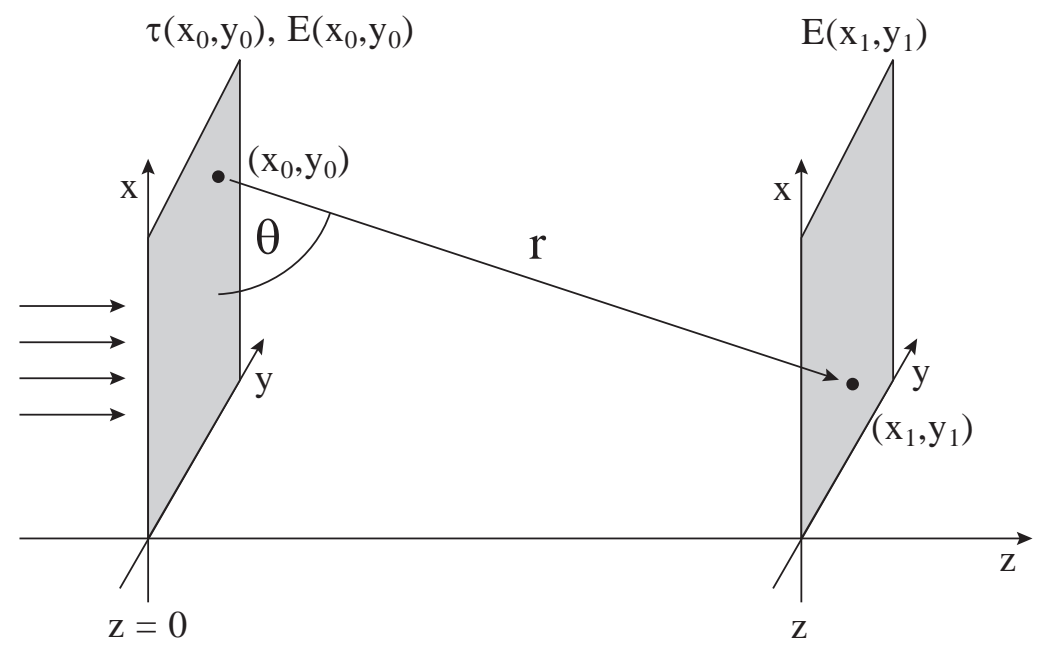

Abbildung 3.18:

Mit dem Kirchhoffschen Beugungsintegral lässt sich ausgehend von Huygensschen Elementarwellen das elektrische Feld in einer beliebigen Ebene $z$ berechnen, dessen Verteilung von einer Transmissions- und Phasenverteilung in der Ebene $z=0$ abhängt. Hierbei wird für jede infinitesimale Flächeneinheit der Feldbeitrag aller bestrahlten Flächeneinheiten berechnet [Lauterborn und Kurz, 2003].

Dabei bezeichnet $\lambda$ die Wellenlänge der Strahlung und $k$ die Wellenzahl. Der Faktor $\cos (\overrightarrow{\mathbf{n}} \overrightarrow{\mathbf{r}})$, der den Winkel zwischen Abstandsvektor $\overrightarrow{\mathbf{r}}$ und der Flächennormalen $\overrightarrow{\mathbf{n}}$ beschreibt, kann bei der im Experiment vorliegenden Geometrie durch die paraxiale Näherung gleich 1 angenommen werden. Das Beugungsintegral ist nicht auf die Beschreibung einer Ebene beschränkt, sondern lässt sich für beliebige Geometrien beschreiben. Dabei müssen stets alle Beleuchtungspunkte berücksichtigt werden. Im zweidimensionalen Fall vereinfacht sich das Beugungsintegral um eine Dimension (siehe zum Beispiel Kalbfleisch [2006]). Dabei werden die elementaren Kugelwellen durch Zylinderwellen ersetzt:

$$
E\left(x_{1}, z\right)=\frac{1}{\sqrt{i \lambda}} \int_{-\infty}^{\infty} E(x, 0) \frac{e^{i k|\overrightarrow{\mathbf{r}}|}}{\sqrt{|\overrightarrow{\mathbf{r}}|}} d x .
$$

Die Propagation des Wellenfelds kann damit sukzessiv in mehreren Ebenen berechnet werden. 


\subsubsection{Modellierung des Experiments}

Das Experiment wird mit einem in Matlab geschriebenem Progamm numerisch simuliert. Dabei wurden zum Betrachten der Einhüllenden stark oszillierender Intensitätsverteilung und der Bestimmung ihrer Halbwertsbreiten zwei Subprogramme verwendet [Wang, 2003; Egan, 2006]. Ferner werden zwei weitere zum graphischen Export der Rechenergebnisse nach LaTeX [Wagenaars, 2006; Schlömer, 2008] verwendet. Das Programm soll dabei nicht nur die optimalen Eigenschaften der Proben wiedergeben, sondern auch die Auswirkungen möglicher Fehler der Probenherstellung und Justage berücksichtigen. Die optisch relevanten Elemente müssen dazu geeignet modelliert und diskretisiert werden. Da durch die Multischicht-Laue-Linse eine Fokussierung nur in einer Raumrichtung erreicht wird, kann das Problem als zweidimensional betrachtet werden. Abbildung 3.19 stellt die verwendeten Parameter in der Simulation dar. Durch Diskretisierung wandelt sich das eindimensionale Kirchhoff-Integral in Gleichung 3.26 in eine numerisch zu berechnende Summe:

$$
E\left(x_{1}, z\right)=\frac{1}{\sqrt{i \lambda}} \sum_{j=1}^{J} E\left(x_{j} 0\right) \frac{e^{i k r_{j}}}{\sqrt{r_{j}}} .
$$

Damit lässt sich das Wellenfeld an allen Stellen nahe der optischen Achse berechnen. Um den Strahlengang aus Kapitel 3.2 zu simulieren, wird zuerst die Röntgenplasmaquelle modelliert. Diese besteht aus einem heißen und dichten Plasma, das in alle Raumrichtungen Strahlung emittiert. Die Quelle wird durch eine zweidimensionale xz-Matrix von typischerweise $1000 \cdot 10$ Punkten diskretisiert. Dabei wird der Beitrag der einzelnen Quellpunkte in jeder Raumrichtung diskret, nach einer Normalverteilung gewichtet, modelliert. Die Phasen der Felder der einzelnen Quellpunkte werden dabei zufällig bestimmt, sodass die Strahlcharakteristik einer inkohärenten Quelle $E_{Q}(x, z)$ entsteht. Da bei einer so zufällig generierten Phasenverteilung in der Quelle dennoch feste Phasenbeziehungen bestehen, wird die gesamte Simulation nicht nur über eine, sondern für eine Vielzahl solcher zufälligen Phasenverteilungen des Wellenfelds berechnet. Abschließend werden die resultierenden Intensitäten der Einzelrealisationen addiert. All diese Eigenschaften entsprechen in guter Näherung den Eigenschaften der experimentell verwendeten Quelle. Damit lässt sich das beleuchtende Feld $E_{B}$ an der Stelle der Multischicht-Laue-Linse, die sich in großer Entfernung befindet, berechnen:

$$
E_{B}\left(x_{B}, z_{B}\right)=\frac{1}{\sqrt{i \lambda}} \sum_{l=0}^{L} \sum_{j=0}^{J} E_{Q}\left(x_{j}, \Delta d_{Q z, l}\right) \frac{e^{i k r_{l, j}}}{\sqrt{r_{l, j}}} .
$$

$\Delta d_{Q z, l}$ bezeichnet die Position der Diskretisierungsebenen entlang der optischen Achse, 
wie in Abbildung 3.19 illustriert, und $L$ und $J$ die Anzahl der Diskretisierungspunkte in $x$ und $z$. Die Multischicht-Laue-Linse wird in äquidistante Punkte diskretisiert. Deren Anzahl bestimmt sich aus der Zahl der Zonen, die hinreichend gut wiedergegeben werden müssen und mit bis zu 15000 Punkten gewählt wurden. Anschließend werden die Amplituden des beleuchtenden Teils des Felds, die sich vor der absorbierenden Zone befinden, auf Null gesetzt. Somit ergeben sich die diffraktiven optischen Eigenschaften der Multischicht-Laue-Linse. Die Maskenfunktion der Multischicht-Laue-Linse kann dabei nicht nur optimal modelliert werden, sondern auch durch mögliche Herstellungsfehler verändert. Das können ungenau strukturierte Schichtdicken, Drift der Schichtdicken und Justagefehler wie Verkippen sein. Anschließend wird das Wellenfeld der MultischichtLaue-Linse in mehreren Ebenen $E_{F}$ um den erwarteten Bildpunkt berechnet. Aus diesen Ebenen ergibt sich ein genaues Bild des Strahlverlaufes und der Güte der Abbildungsleistung:

$$
\sum_{l=0}^{l} E_{F}\left(x_{F}, z_{F, l}\right)=\frac{1}{\sqrt{i \lambda}} \sum_{j=0}^{J} E_{B, M L L}\left(x_{j}, z_{B}\right) \frac{e^{i k r_{j}}}{\sqrt{r_{j}}} .
$$

Zusammenfassend ist der Rechenweg in Abbildung 3.20 noch einmal grafisch veranschaulicht. Die komplexen Feldstärken werden als Intensitäten durch Multiplikation mit ihrem komplex Konjugiertem dargestellt.

Zur Bestimmung der Strahlparameter kann die Kaustik aus den Strahlbreiten mit verschiedenen Methoden bestimmt werden. Dazu zählen eine einfache Anpassung der Normalverteilung und die Methode der zweiten Momente, die bei stark feinstrukturierten Strahlen verwendet wurde.

In der Literatur befassen sich Kopylov u. a. [1995, 1996] und Kurokhtin und Popov [2002] ebenfalls mit der Simulation von Zonenplatten. Ihr Ansatz mit Hilfe der parabolischen Wellengleichung ermöglicht, das Feld innerhalb einer räumlich ausgedehnten Zonenplatte näher zu untersuchen und so die Zonenplattengeometrie zu optimieren. Bei einer modellhaften Zonenplatte für 2,4 nm Wellenlänge kommen sie zu dem Ergebnis, dass insbesondere die Effizienz der Optik durch die räumliche Ausdehnung der Zonenplatte beeinträchtigt wird [Kopylov u. a., 1995]. 

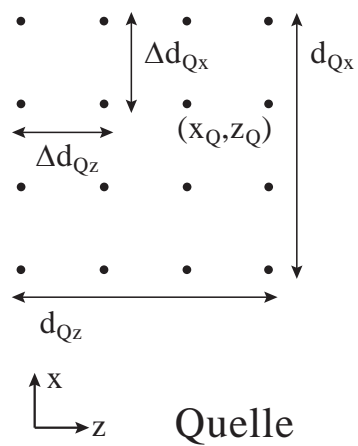

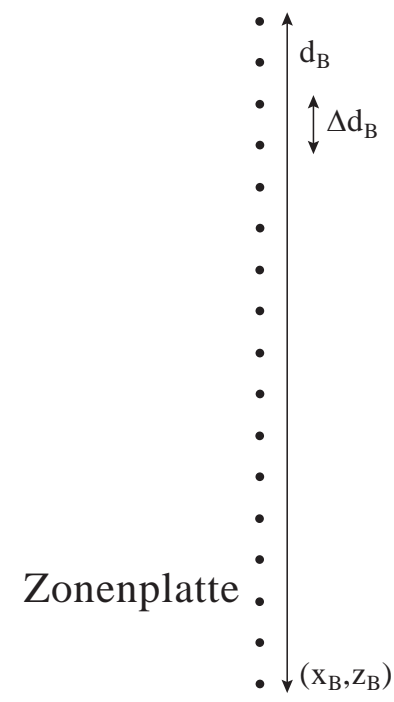

\section{Fernfeld}

Abbildung 3.19:

Benennungen der in dem Simulationsprogramm (Anhang C auf Seite 135) verwendeten Größen. Der Versuchsaufbau kann zweidimensional modelliert werden. Die räumlich ausgedehnte Quelle wird in einer Matrix aus Einzelquellpunkten diskretisiert. Die ebenfalls diskretisierte Beleuchtung der Optik wird in der Ebene der Zonenplatte berechnet und von dort weiter in eine Matrix propagiert, die um den erwarteten Bildpunkt gelegt ist.

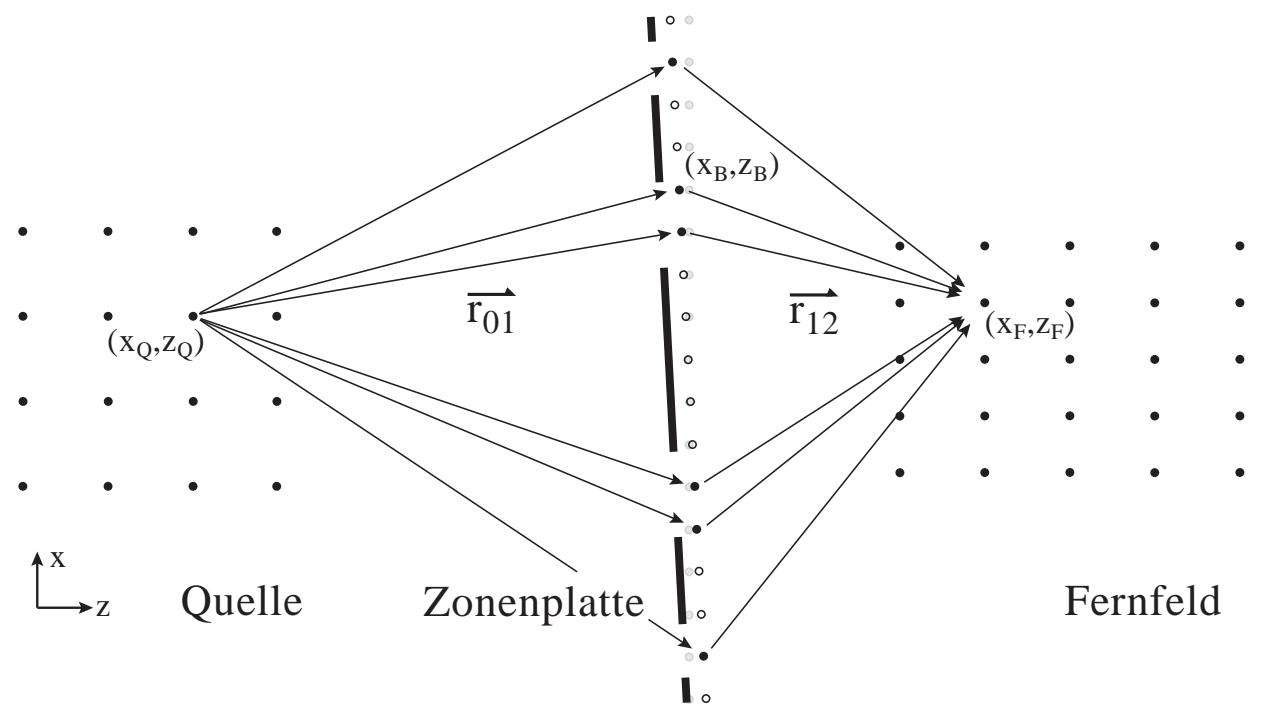

\section{Abbildung 3.20:}

Prinzipieller Berechnungsweg des diskretisierten Kirchhoff'schen Beugungsintegrals im Simulationsprogramm. Zunächst wird das Feld für alle Punkte in der Beleuchtungsebene aus den Quellpunkten berechnet. Diese kann dabei die optische Achse in einem beliebigen Winkel schneiden. Anschließend setzt eine Maskenfunktion die Amplitude aller nicht transmittierenden Bereiche der Optik auf Null. In einem zweitem Schritt wird die Kirchhoff-Summe für die verschiedenen Ebenen hinter der Zonenplatte berechnet. 
Tabelle 3.5:

Ablenkwinkel des als Teststruktur gerechneten Gitters. Es zeigt sich eine sehr gute Übereinstimmung mit den analytisch berechneten Werten.

\begin{tabular}{lcc}
\hline & 1. Ordnung & 3. Ordnung \\
\hline Simulation & $0,92^{\circ}$ & $2,74^{\circ}$ \\
Gittergleichung & $0,92^{\circ}$ & $2,74^{\circ}$ \\
\hline
\end{tabular}

\subsubsection{Simulationsergebnisse eines Gitters als Teststruktur}

Um die Leistungsfähigkeit der Simulation und ihre physikalische Richtigkeit zu überprüfen, wird zunächst die Strahlpropagation eines einfachen Gitters berechnet um dieses Simulationsergebnis mit dem in diesem Fall bekannten analytischen Ergebnis zu vergleichen. Dazu wurden mit dem Experiment vergleichbare Parameter gewählt, eine ausgedehnte gaußverteilte Quelle $\left(300 \mathrm{~nm} 1 / e^{2}\right)$ bei $2,88 \mathrm{~nm}$ Wellenlänge und $80 \mathrm{~cm}$ Abstand von dem $2 \mu \mathrm{m}$ breiten Gitter.

Bei einem optischen Gitter unter senkrechtem Strahlungseinfall ergeben sich die Beugungsmaxima durch ganzzahlige Vielfache der Wellenlänge $\lambda$ und der Gitterkonstanten $g$, die mit $200 \mathrm{~nm}$ gewählt wird, wobei Stege und Spalten jeweils $100 \mathrm{~nm}$ breit sind. Die Lage der Maxima wird dann durch die Gittergleichung gegeben:

$$
\sin (\phi)=\frac{n \cdot \lambda}{g}
$$

In Abbildung 3.21 sind die Intensitäten der Propagation des Wellenfelds über $5 \mathrm{~mm}$ aufgetragen, die die für ein Gitter typische Beugungserscheinung zeigen. Die darauf folgende Abbildung 3.22 zeigt die Lage der Maxima und deren Intensitäten auf einem virtuellen Schirm in eben dieser Entfernung. Werden die Ablenkwinkel aus den Schwerpunkten der Beugungsmaxima berechnet, so ergibt sich eine gute Übereinstimmung mit den analytisch berechneten Werten. Wie bei dieser Anordnung erwartet, verschwindet dabei die zweite Beugungsordnung (siehe beispielsweise Barkusky u. a. [2007]) durch das Minimum der die Gittergleichung einhüllenden Spaltfunktion. Die Ablenkwinkel aus Simulation und Berechnung sind für die ersten Beugungsordnungen in Tabelle 3.5 zusammengefasst. Auch der Abfall der Intensität des ersten Beugungsmaximums auf $40 \%$ des Niveaus des nullten Beugungsmaximums entspricht der Erwartung [Attwood, 1999]. Diese Simulation zeigt, dass von einer guten Validität des Programms ausgegangen werden kann. Es ist geeignet für eine qualitative und quantitative Simulation der Wellenfelder hinter den Multischicht-Laue-Linsen. 


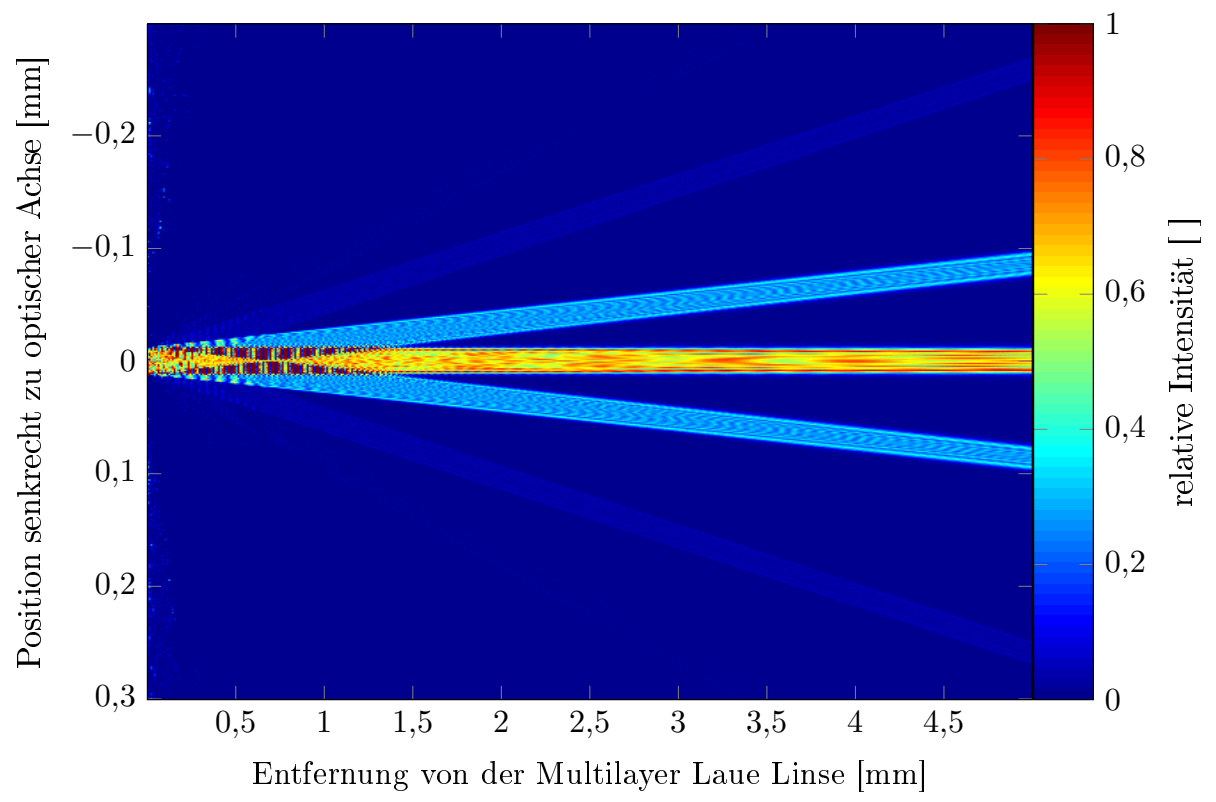

Abbildung 3.21:

Auf das Maximum normierte Intensitäten des Wellenfelds hinter einem optischen Gitter über eine Realisation. Da Stege und Spalten gleich breit sind, wird die 2. Beugungsordnung unterdrückt.

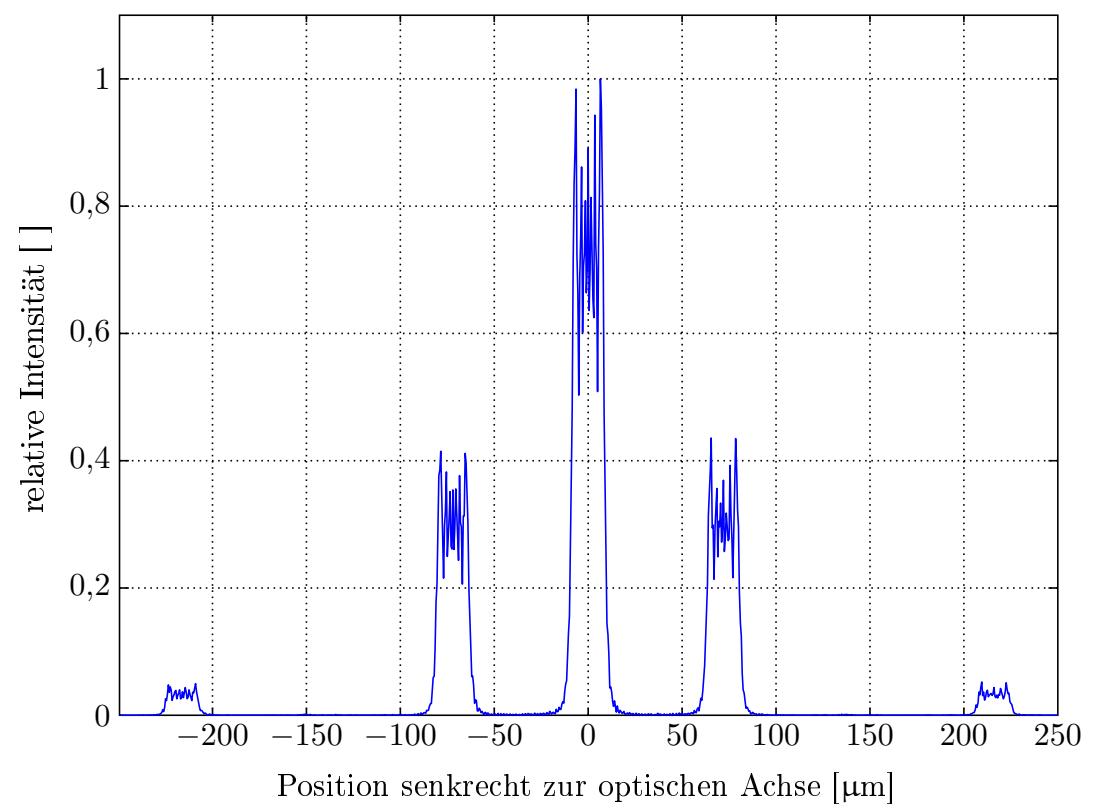

Abbildung 3.22:

Lage der Beugungsordnungen auf einem $5 \mathrm{~mm}$ weit entfernten Schirm. Aus den Schwerpunkten der abgelenkten Beugungsordnungen werden die Ablenkwinkel bestimmt. 


\subsubsection{Simulationsergebnisse für Multischicht-Laue-Linse 1a}

Die Simulation wird für die verschiedenen hergestellten Proben durchgeführt. Dabei ist die zuerst untersuchte Probe 1 ein System zweier identischer Schichtpakete, welche gegenüber liegend einen gemeinsamen Linienfokus erzeugen sollen. Sie ist eine eindimensionale Zonenplatte, die nur einen Teilbereich der theoretisch berechneten Zonenplatte darstellt. Da die transmittierenden Schichten nicht auf der optischen Achse liegen handelt es sich um eine ,off-axis“-Zonenplatte. Die kleinste und größte strukturierte Schicht sowie die Apertur und Brennweite sind in Tabelle 3.1 zusammengefasst.

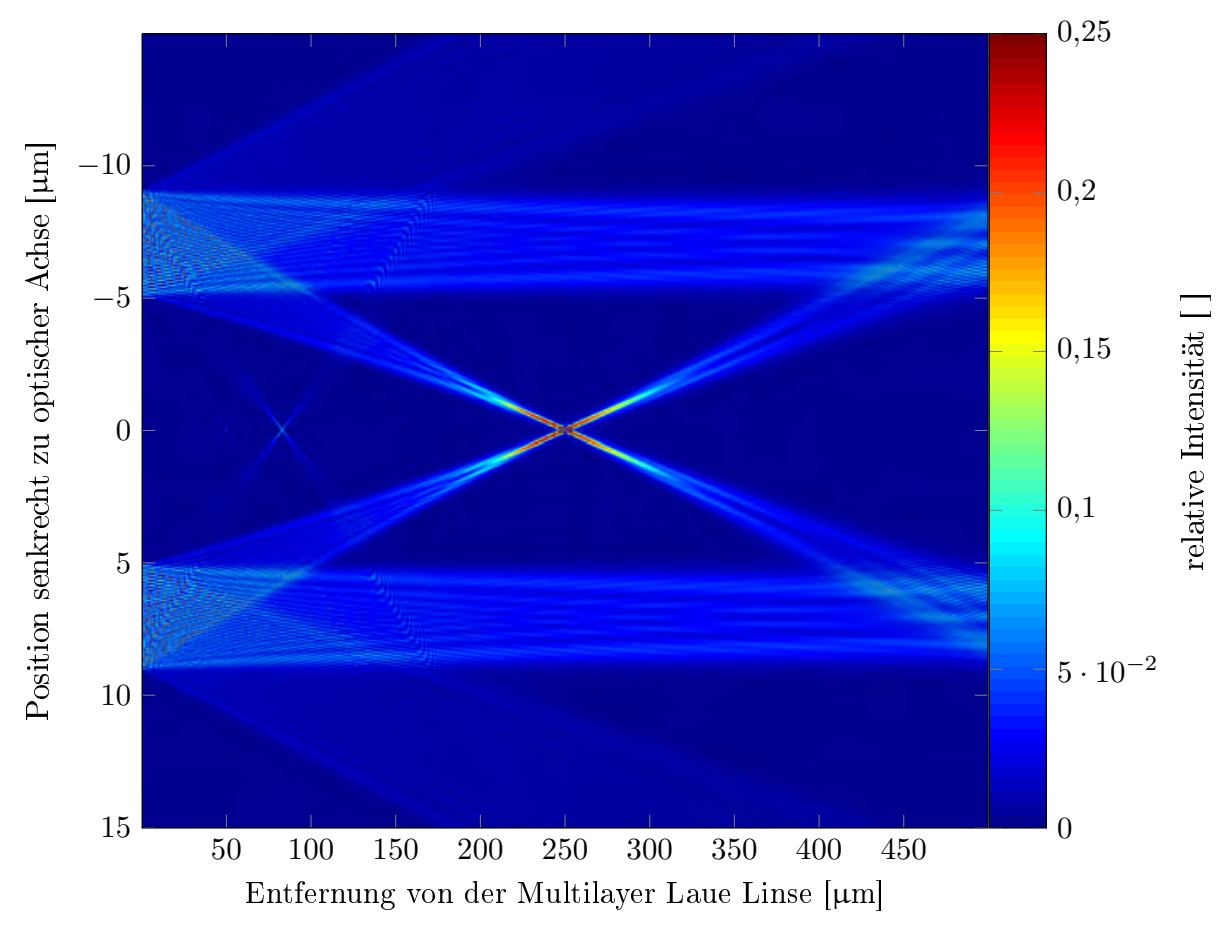

Abbildung 3.23:

Intensitäten des Wellenfelds aus der Simulation hinter der Multischicht-Laue-Linse 1a in einer einzelnen Realisation. Deutlich zu erkennen ist die transmittierte und nicht gebeugte 0. Beugungsordnung sowie die sich in einem gemeinsamen Bildpunkt treffenden fokussierenden Beugungsordnungen. Eine Abschätzung der mittleren Intensität in der Zonenplattenebene und der maximalen Intensität im Fokus zeigt eine Intensitätsverstärkung von $1 \mathrm{zu}$ 4. Ferner sind auch die divergenten Beugungsordnungen und Bildpunkte höherer Beugungsordnungen zu erkennen.

Mit dem im Kapitel 3.3.2 beschriebenen Programm wird das Feld hinter der Multischicht-Laue-Linse 1a über die Länge der doppelten Brennweite berechnet. Die Parameter der Simulation werden dabei so gewählt, dass sie dem durchgeführten Experiment möglichst exakt entsprechen. Die Ergebnisse für die aus den Simulationen bestimmten minimalen Strahlbreiten sind in Tabelle 3.7 zusammenfassend abgebildet. 
Tabelle 3.6:

Modellierungsparameter der MLL Simulationen. Diese sind so gewählt, dass sie den experimentellen Parametern entsprechen. Die Linsenparameter sind entsprechend Tabelle 3.1 gewählt.

\begin{tabular}{lccc}
\hline & $\begin{array}{c}\text { Größe Plasma } x \\
\left(1 / e^{2}\right)\end{array}$ & $\begin{array}{c}\text { Größe Plasma } z \\
\left(1 / e^{2}\right)\end{array}$ & $\begin{array}{c}\text { Abstand } \\
\text { Quelle-MLL } d_{01}\end{array}$ \\
\hline $\begin{array}{l}\text { MLL Probe 1 } \\
2 \text { Schichtpakete }\end{array}$ & $300 \mu \mathrm{m}$ & $300 \mu \mathrm{m}$ & $40,80,120 \mathrm{~cm}$ \\
$\begin{array}{l}\text { MLL Probe 1 } \\
\text { 1 Schichtpaket }\end{array}$ & $300 \mu \mathrm{m}$ & $300 \mu \mathrm{m}$ & $80 \mathrm{~cm}$ \\
MLL Probe 2 & $300 \mu \mathrm{m}$ & $300 \mu \mathrm{m}$ & $80 \mathrm{~cm}$ \\
MLL Voll & $300 \mu \mathrm{m}$ & $300 \mu \mathrm{m}$ & $80 \mathrm{~cm}$ \\
\hline
\end{tabular}

Die aus dem Wellenfeld berechnete Intensitätsverteilung von Probe 1 ist in Abbildung 3.23 dargestellt. Dabei können alle in der Theorie erwarteten und bekannten Eigenschaften beobachtet werden: Neben der transmittierten 0. Beugungsordnung erkennt man deutlich die 1. Beugungsordnungen. Die beiden fokussierenden Beugungsordnungen treffen sich an ihrer schmalsten Stelle in einem gemeinsamen Punkt. Gleiches gilt für die Bildpunkte der höheren Beugungsordnungen, die in kürzeren Abständen hinter der Optik liegen. Die divergenten Beugungsordnungen werden von der optischen Achse weggebeugt. Durch die Überlagerung der beiden fokussierenden Beugungsordnungen ergibt sich durch Interferenz im Bildpunkt ein feinstrukturiertes Bildpunktprofil, wie es in der Detailsimulation in Abbildung 3.24 gezeigt wird. Die Breite dieses Strahlprofils wurde mit der Methode der zweiten Momente bestimmt. Dabei werden alle Intensitäten mit weniger als $5 \%$ der Intensität des Hauptmaximums unterdrückt, um den Auswertungsbereich räumlich zu begrenzen. Die Ergebnisse dieser Auswertung sind in Abbildung 3.25 aufgetragen. Die Strahltaille hat ihr Minimum von $139,8 \mathrm{~nm}$ bei $250,5 \mu \mathrm{m}$ Bildpunktweite. Das entspricht beinahe dem Designparameter.

Um ein tieferes Verständnis der gewonnenen Messdaten, die mit $375 \pm 30 \mathrm{~nm}$ deutlich über dem in der Simulation bestimmten Wert liegen, zu erlangen, werden Störeinflüsse und Abweichungen vom optimalen Experiment simuliert. Zunächst wird, da es sich bei dem durchgeführten Experiment um eine Abbildung handelt, überprüft, ob der Abstand des ausgedehnten Quellplasmas einen Einfluss auf die im Experiment bestimmte Größe des Bildpunkts hat. Die Größe des Quellplasma von $300 \mathrm{~nm}$ wird dabei so parametrisiert, wie es mit einer Lochkamera [Kranzusch u. a., 2003; Reese u. a., 2011] beobachtet wurde. Ein Lochkameraaufnahme eines im Experiment typischen Plasmas ist bereits in Abbildung 2.2(a) auf Seite 27 gezeigt. 


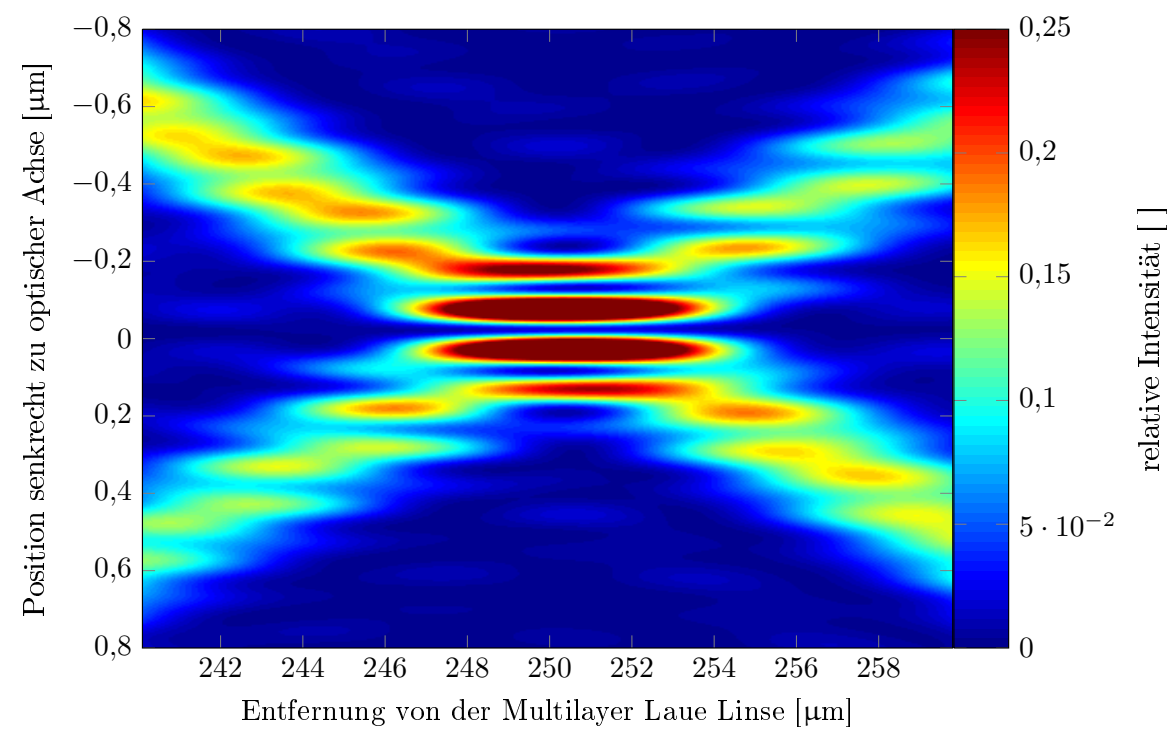

Abbildung 3.24:

Detailsimulation des Bildpunkts hinter der Multischicht-Laue-Linse 1a über 1000 Realisationen. Deutlich zu erkennen ist eine Feinstrukturierung des Bildpunkts, die durch eine relativ geringe Anzahl von Zonen und Überlagerung der Teilstrahlen hervorgerufen wird.

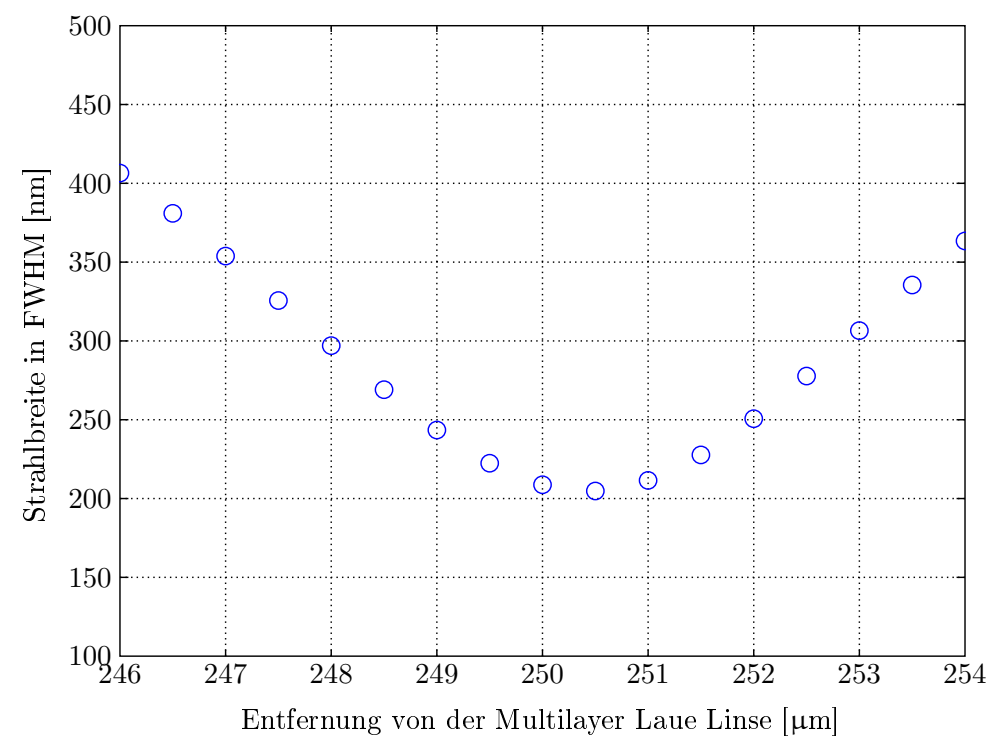

Abbildung 3.25:

Strahlbreiten nahe des Bildpunkts bei der Multischicht-Laue-Linse 1a. Die Breiten des Strahls sind wegen seiner starken Strukturierung nach der Methode der zweiten Momente aus der Kaustik in Abbildung 3.24 bestimmt. Die geringe Abweichung der Taillenlage von $500 \mathrm{~nm}$ wird infolge der durch die Simulation noch nicht vollständig randomisierten Phasenzusammenhänge verursacht. 
Zunächst werden die Profile der Bildpunkte dreier identischer Simulationen lediglich unter verschiedenen Abständen einer kohärenten Punktquelle in Abbildung 3.26 gezeigt. Um die Amplituden dieser Kurven miteinander vergleichen zu können, wird ein Korrekturfaktor für den Quellabstand $d_{01}$ von $1 / d_{01}$ eingeführt, der berücksichtigt, dass die Plasmaquelle als $4 \pi$ Strahler in alle 3 Raumrichtungen emittiert, wobei eine in der Simulationsgeometrie nicht berücksichtigt wird. Die Optik ist auf eine minimale Fokussierung von etwa $128 \mathrm{~nm}$ begrenzt. Die Intensität fällt wie erwartet mit dem Quadrat des Abstands ab. In der anschließenden Simulation wird die Punktquelle durch die ausgedehnte, inkohärente Quelle ersetzt. Die Bildpunktprofile dieser Simulation sind in Abbildung 3.27 aufgetragen. Neben der nun verschmierten und leicht verschobenen Feinstruktur des Strahlprofils ist deutlich erkennbar, dass bei einem kleinen Quellabstand von $40 \mathrm{~cm}$ der Betrag der minimalen Strahltaillenbreite vergrößert wird. Bei einem Abstand von $80 \mathrm{~cm}$ entspricht die minimale Strahltaillenbreite beinahe der bei Punktquellenbeleuchtung. Unter einem größeren Quellabstand von $120 \mathrm{~cm}$ tritt keine weitere Verkleinerung ein, lediglich die Intensität des Fokus nimmt weiter ab. Eine Zusammenstellung aller minimalen Strahltaillenbreiten findet sich in Tabelle 3.7. Die Ausdehnung des Quellplasmas hat in diesem Experiment also keine negative Auswirkung auf die minimalen Strahltaillenbreite. Ein größerer Quellabstand $d_{01}$ führt zu keiner Verbesserung der Abbildungsleistung.

Die Untersuchung weiterer Fehlerquellen soll das optische Verhalten der Probe 1a besser erklären. Eine detailierte Zusammenfassung aller bekannten experimentellen Fehler findet sich in Kapitel 3.4 auf Seite 99.

Die bisher als optimal hergestellt und justiert angenommene Probe 1 wird durch die Simulation mit verschiedene Störungen, wie sie im Experiment auftreten können, verfälscht. Ein möglicher Justagefehler wird mit dem Programm durch Verkippen der Optik simuliert. Die Wahl der Größe des Verkippungswinkels mit $2^{\circ}$ und $4^{\circ}$ orientiert sich dabei an einer Abschätzung, die am Versuchsaufbau durch die gegebenen Justagemöglichkeiten gemacht wurde. Die fokussierenden Eigenschaften der Linse ändern sich dabei nur wenig: Die Breite des Bildpunkts bleibt weitgehend konstant. Im Detail kann beobachtet werden, dass sich die Brennweite um einige $100 \mathrm{~nm}$ verkürzt. Dies ist darauf zurückzuführen, dass die Projektion der Zonen auf die Ebene senkrecht zur optischen Achse die Schichten der Multischicht-Laue-Linse kleiner erscheinen lässt. Auch verlagert sich der Bildpunkt einige $100 \mathrm{~nm}$ leicht von der optischen Achse weg und verändert dabei geringfügig seine Feinstruktur. Beide Effekte sind unter inkohärenten Beleuchtungsbedingungen nicht eindeutig auszumachen. Allerdings sind sie unter kohärenter Punktquellenbeleuchtung deutlich zu erkennen. Zusammengefasst hat diese Form der Dejustage keinen entscheidenden Einfluss auf die fokussierenden Eigenschaften der Multischicht-Laue-Linse. Die Ergebnisse für verschiedene Verkippungswinkel sind mit den anderen Ergebnissen in der Tabelle 3.7 zusammengefasst. Das Auflösungsvermögen der Multischicht-Laue-Linse 
wird demnach nicht durch die Konzeption oder Justage des Experiments limitiert. Dennoch wird im Experiment eine deutlich größere als die simulierte Strahltaillenbreite gemessen. Daher werden verschiedene Fehler, wie sie in der Herstellung der Proben nach Krebs [2011] aufgetreten sind, in der Modellierung der Zonen zusätzlich berücksichtigt. Aufnahmen der Probe mit dem Rasterelektronenmikroskop zeigen, dass die Schichten kontinuierlich in aufsteigender Ordnungszahl dünner strukturiert wurden als im Probendesign vorgesehen. Die innerste Schicht ist etwa $11 \%$ dünner als vorgesehen, in Abbildung 3.29 ist dieses illustriert.

Der Herstellungsfehler wird durch eine lineare Zonenbreitenverdünnung modelliert. Die innerste Schicht der Multischicht-Laue-Linse entspricht dabei einer Brennweite von $200 \mu \mathrm{m}$, während die äußerste einer Brennweite von $250 \mu \mathrm{m}$ entspricht. Der Abstand der Schichtpakete zueinander ist somit nicht mehr klar nach dem Zonenplattenbildungsgesetz definiert und wurde bei der Herstellung an der dünnsten Zonenbreite orientiert. Die Abbildung 3.28 zeigt die massive Verschlechterung der Abbildungsleistung nahe des designten Bildpunkts. Am Schnittpunkt der fokussierenden Beugungsordnungen wird eine gemeinsame Strahltaillenbreite von etwa 1,7 $\mu \mathrm{m}$ bestimmt, das verglichen mit der optimal modellierten Probe einer Verbreiterung um den Faktor 13 entspricht. Die lineare Verfälschung der Zonenbreiten verursacht also eine extreme Aberration und wird für ein einzelnes Schichtpaket in Kapitel 3.3.5 näher untersucht, da, wie im Experiment, die minimale Strahltaillenbreite, nicht mit der des gegenüberliegenden Schichtpakets zusammenfällt und somit allein zu betrachten ist. 


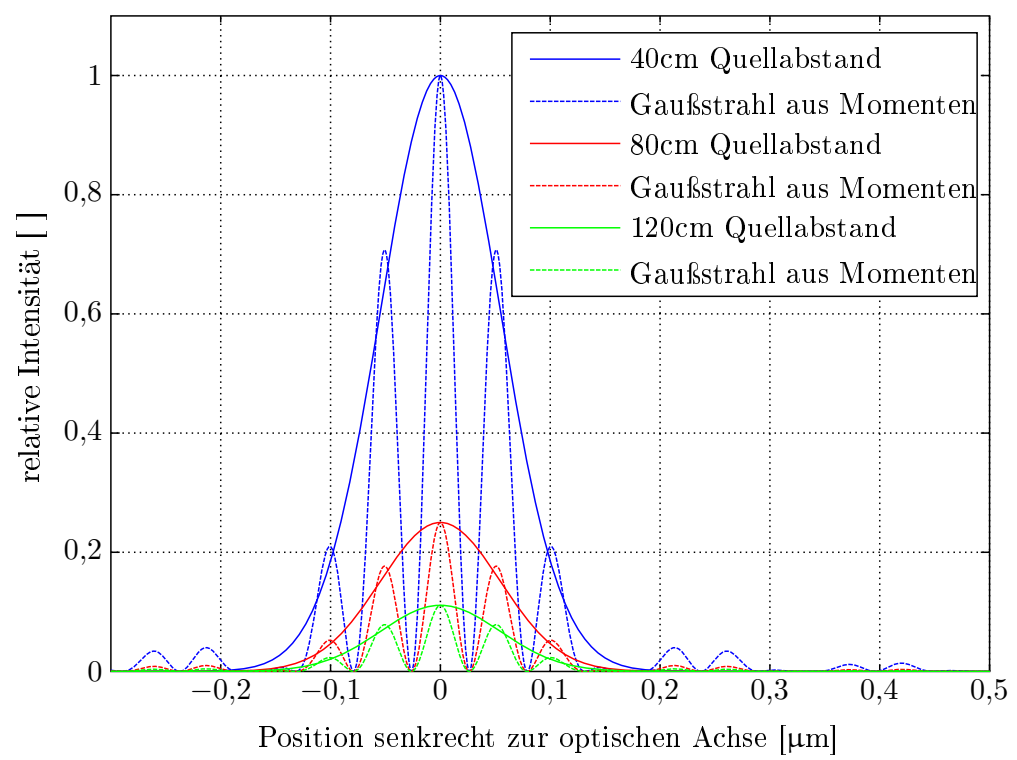

Abbildung 3.26:

Vergleich der Breite und der Intensität der Bildpunkte in Abhängigkeit des Abstands der Optik von einer angenommenen kohärenten Punktquelle. Die zu den Momentenbreiten assoziierte Normalverteilung ist mit aufgetragen. Deren Halbwertsbreiten finden sind in Tabelle 3.7. Die Intensitäten fallen, wie zu erwarten ist, mit dem Abstandsquadrat ab.

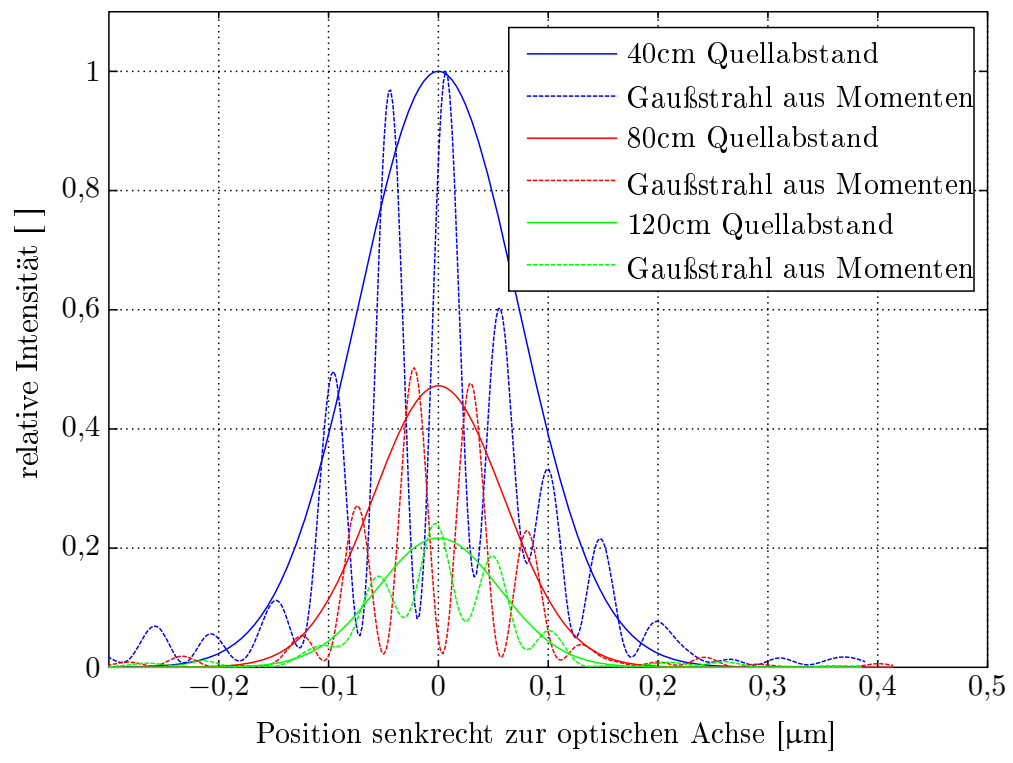

Abbildung 3.27:

Vergleich der Breite und der Intensität der Bildpunkte in Abhängigkeit des Abstands der Optik von der Plasmaquelle. Die einzelnen Maxima der Feinstruktur, die aus der Überlagerung der beiden Teilstrahlen herrührt, schieben abhängig von der inkohärenten Phasenverteilung die Lage der Quelle leicht. Die zu den Momentenbreiten assoziierte Normalverteilung ist mit aufgetragen, ihre Maxima sind Übereinander dargestellt. Die Halbwertsbreiten finden sind in Tabelle 3.7. 


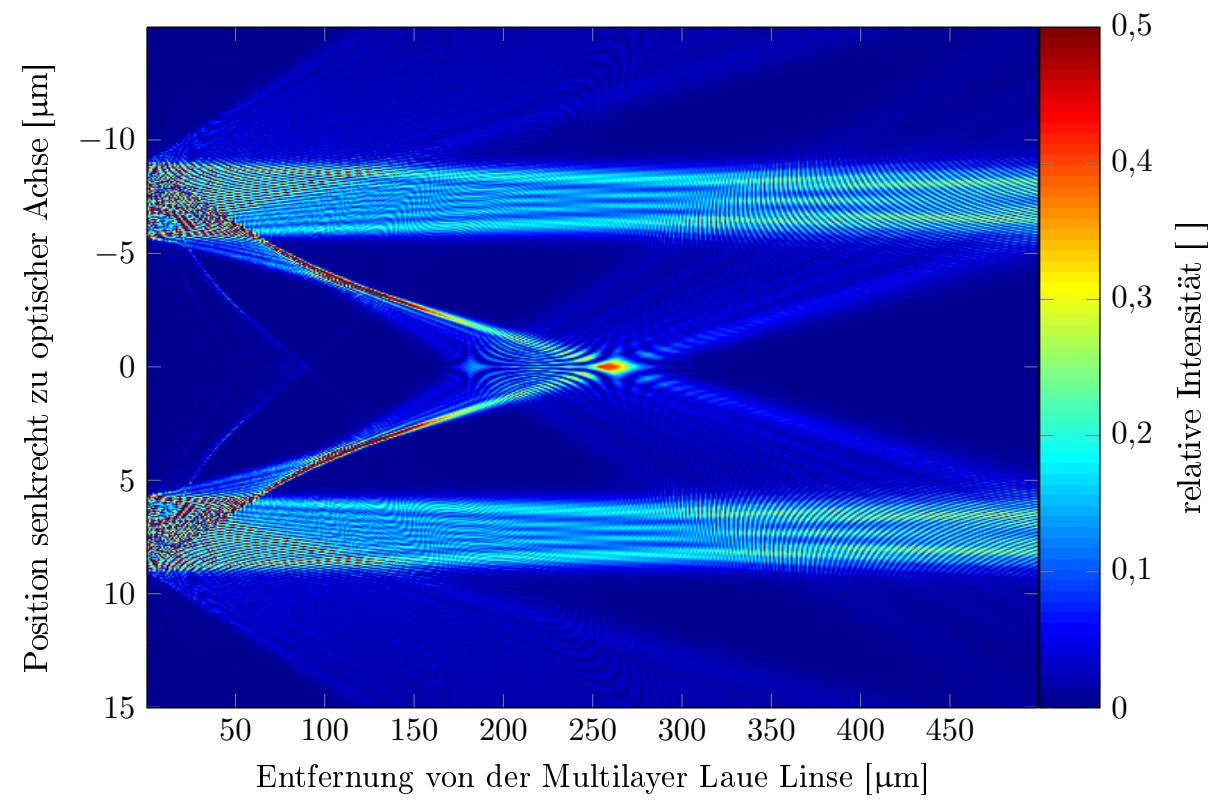

Abbildung 3.28:

Simulation des Wellenfelds hinter einer fehlerbehaften Probe 1. Die bekannten Herstellungsmängel der Probe wurden in dieser Simulation bestmöglich berücksichtigt.

\section{Beschichtungsrichtung}

a)
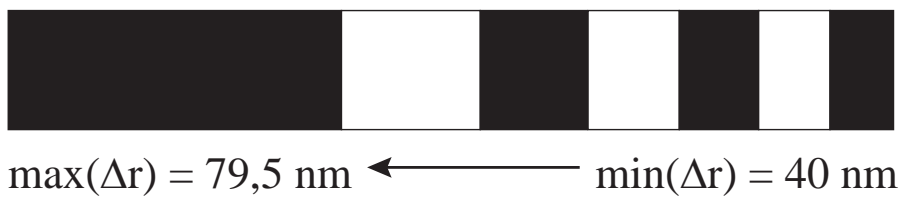

$\mathrm{a}=4,5 \mu \mathrm{m}$

b)

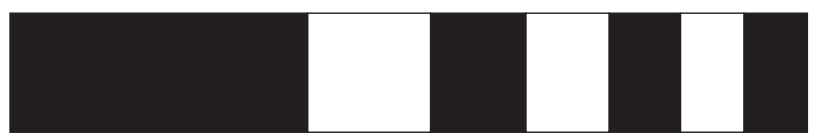

$$
\begin{array}{r}
\max (\Delta \mathrm{r})=70,8 \mathrm{~nm} \longleftarrow \min (\Delta \mathrm{r})=40 \mathrm{~nm} \\
\mathrm{a}=3,5 \mu \mathrm{m}
\end{array}
$$

\section{Abbildung 3.29:}

In Strukturierungsrichtung weisen die Zonen einen zunehmenden Fehler auf und werden zunehmend dünner. In der Simulation wird diese Abnahme als linear angenommen. (a) Zonenplatte nach Designparametern. (b) Die dünnste Zone stimmt mit dem Designparameter überein, während die dickste stark davon abweicht. 


\subsubsection{Simulationsergebnisse für Multischicht-Laue-Linse 1b (Einzelschichtsystem)}

Die beiden Schichtpakete von Multischicht-Laue-Linse 1a erzeugen wegen der starken Aberration keinen gemeinsamen Bildpunkt. So wird, wie auch schon bei der Auswertung der Experimente (Kapitel 3.2.2), die fokussierende Beugungsordnungen für ein einzelnes Schichtpaket (Multischicht-Laue-Linse 1b) untersucht. Es werden also die Strahlparameter der einzelnen fokussierenden Beugungsordnung näher bestimmt. Die zunächst optimalen optischen Eigenschaften eines solchen Schichtpakets im Experiment sind in Abbildung 3.30 dargestellt. Sie verhalten sich analog zu den Ergebnissen der Simulation der Doppelschichtpakete. Der Bildpunkt weist jedoch keine derart stark ausgeprägte Feinstruktur auf (Abbildung 3.24), da die Überlagerung mit dem Strahl des zweiten Schichtpakets entfällt. Eine Analyse des Strahlprofils (Abbildung 3.32) zeigt, dass die Fokussierung des Strahls etwa über einen Bereich von $20 \mu \mathrm{m}$ beinahe konstant ist. An seiner schmalsten Stelle hat die Strahltaille (Profil in Abbildung 3.33) eine Momentenbreite von $182 \mathrm{~nm}$.

Bei einer Simulation eines Einzelschichtsystems der fehlerbehafteten Probe 1b werden die starken Aberrationen endgültig offenbar (Abbildung 3.34). Die Strahltaille liegt nicht mehr in der Nähe der vorgesehenen Brennweite von $250 \mu \mathrm{m}$, sondern bei etwa $120 \mu \mathrm{m}$. Dieses verdeutlicht eine Analyse der Strahlbreiten nach Momentenmethode in Abbildung 3.35. Eine genaue Darstellung des Strahlprofils an der Strahltaille in Abbildung 3.36 zeigt einen stark feinstrukturierten Strahl. Dessen genaue Parameter können nicht durch eine Anpassung der Normalverteilungsfunktion beschrieben werden. Die Strahlbreite wird daher mittels der Methode der zweiten Momente bestimmt. Dabei werden zur Begrenzung des auszuwertenden Bereichs Intensitäten von weniger als $5 \%$ der Intensität des Hauptmaximums unterdrückt. Die zu diesen Parametern korrespondierende Normalverteilung ist zur Veranschaulichung neben dem Strahlprofil aufgetragen. Es zeigt sich, dass die zugehörige Halbwertsbreite des Strahls mit $456 \mathrm{~nm}$ deutlich über der der Strahltaille einer optimalen Probe und im Bereich der Schneidblendenrastermessung von $375 \pm 30 \mathrm{~nm}$ liegt.

Zusammenfassend lässt sich feststellen, dass Probe 1 durch die umfangreichen Simulationen gut verstanden ist. Wenngleich die bekannten Fehler im Herstellungsprozess nur näherungsweise modelliert sind, reproduzieren sie qualitativ und auch quantitativ gut die Messwerte der Schneidblendenrastermessung und des Divergenzwinkelexperiments. Eine genauere Übereinstimmung ist angesichts von Messungenauigkeiten und Detailwissen um die exakten einzelnen Schichtdicken unwahrscheinlich, da die Modellierung mit einer linearen Abweichung nur eine Näherung darstellt. Dennoch erklärt schon diese lineare Abweichung die im Experiment erhobenen Messdaten gut, sodass davon ausgegangen werden kann, dass die Abweichung von den erwarteten Werten für eine optimale 
Multischicht-Laue-Linse im Wesentlichen auf Schwierigkeiten bei der Schichtdickenrealisation zurückzufüren ist.

\subsubsection{Simulationsergebnisse für Multischicht-Laue-Linse 2}

Die Herstellung der Probe 2 (Parameter siehe Tabelle 3.1) erfolgte mit dem Ziel, ein besonders genau definiertes Schichtpaket herzustellen, um die Leistungsfähigkeit der Methode auch für kleine Zonen im Bereich von $10 \mathrm{~nm}$ zu demonstrieren. Dieses geschieht auf Kosten der Aperturgröße und damit des Auflösungsvermögens der Optik [Vinogradov und Mitrofanow, 2009]. Die Intensitäten des Wellenfelds nahe der Strahltaille hinter der optimalen Probe sind in Abbildung 3.37 aufgetragen.

Die theoretisch zu erwartende Breite der Strahltaille (Abbildung 3.38) ist wegen oben genannter Gründe größer als die bei Probe 1 und beträgt etwa $252 \mathrm{~nm}$ im Abstand von $66 \mu \mathrm{m}$ hinter der Probe. Sie liegt damit deutlich vor dem vorgesehenem Bildpunkt bei $100 \mu \mathrm{m}$, bei dem die Strahlbreite bei $308 \mathrm{~nm}$ liegt. Dies kann, wie Vergleichssimulationen zeigen, mit der relativ geringen Zahl der Zonen und der Aperturgröße erklärt werden.

Das Verhalten bei Verkippen der Linse zur optischen Achse ist weitgehend analog zu dem Verhalten von Probe 1 und beeinträchtigt das Auflösungsvermögen kaum.

\subsubsection{Simulationsergebnisse für Multischicht-Laue-Linse, Volllinse}

Mit dem Simulationsprogramm sollten die Anforderungen an ein Laborexperiment ermittelt werden, mit dem eine Beleuchtung von deutlich weniger als $100 \mathrm{~nm}$ erreicht werden kann. $\mathrm{Zu}$ beachten ist dabei der Abbildungsmaßstab des Plasmas, der das Auflösungsvermögen limitiert. Bei der Plasmagröße des Gastargets von $210 \mu \mathrm{m}$ (FWHM) Durchmesser und $80 \mathrm{~cm}$ Abstand einer Multischicht-Laue-Linse mit $250 \mu \mathrm{m}$ Brennweite von der Quelle ist das Auflösungsvermögen auf $65 \mathrm{~nm}$ begrenzt. Um die Strahltaille weiter zu verkleinern, muss die Plasmagröße durch geeignete Targetkonzepte verkleinert, die Distanz des Plasmas erhöht oder die Brennweite verkürzt werden. Eine einfache Erhöhung des Quellabstands auf $200 \mathrm{~cm}$ verkleinert den möglichen Bildpunkt auf $26 \mathrm{~nm}$. Die Kaustik der Intensitäten des Wellenfelds einer fiktiven Vollzonenplatte mit 200 Zonen ist in Abbildung 3.39 dargestellt. Abbildung 3.40 zeigt eine Detailsimulation des Bildpunkts. Der Bildpunkt dieser Abbildung misst nur $40 \mathrm{~nm}$ nach der Momentenmethode in der Halbwertsbreite. Ein Schnitt durch die Strahltaille des simulierten Strahls ist in Abbildung 3.41 dargestellt. 


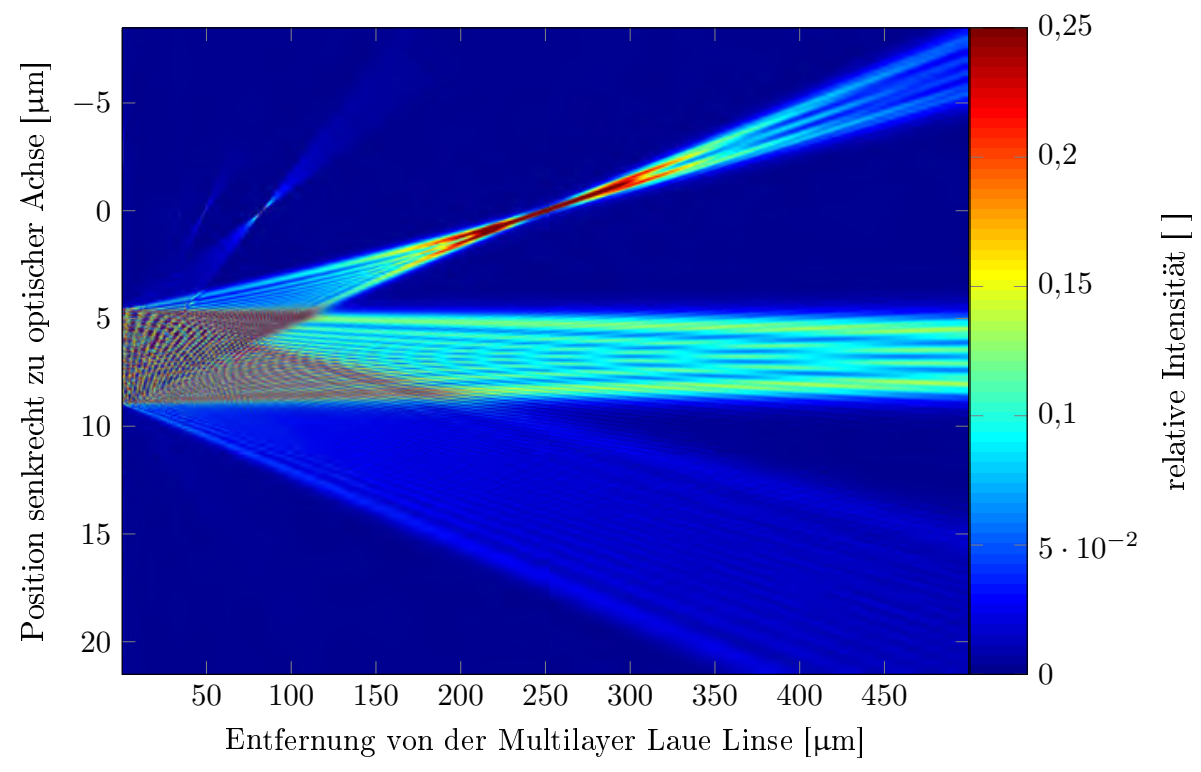

Abbildung 3.30:

Simulation der Intensität hinter einem einzelnen, idealen Schichtpaket von Multischicht-Laue-Linse 1b über 1000 Realisationen. Eine Abschätzung der mittleren Intensität in der Zonenplattenebene und der maximalen Intensität im Fokus zeigt eine Intensitätsverstärkung von 1 zu 0,8. Deutlich erkennbar ist die transmittierten, fokussierten und divergierenten Beugungsordnungen sowie die Bildpunkte mehrerer Beugungsordnungen.

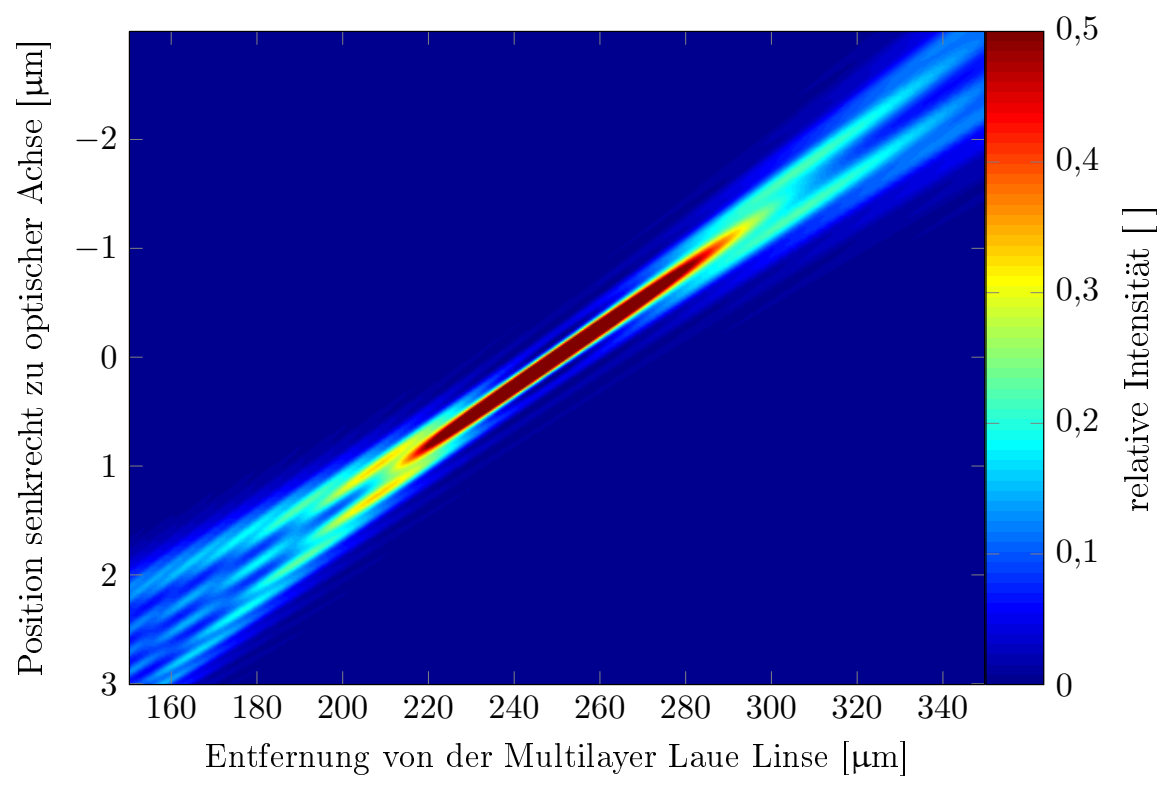

Abbildung 3.31:

Detailsimulation der Strahltaille aus Abbildung 3.30 über 1000 Realisationen. Der Strahl verjüngt sich um die vorgesehene Bildpunktweite von $250 \mu \mathrm{m}$. 


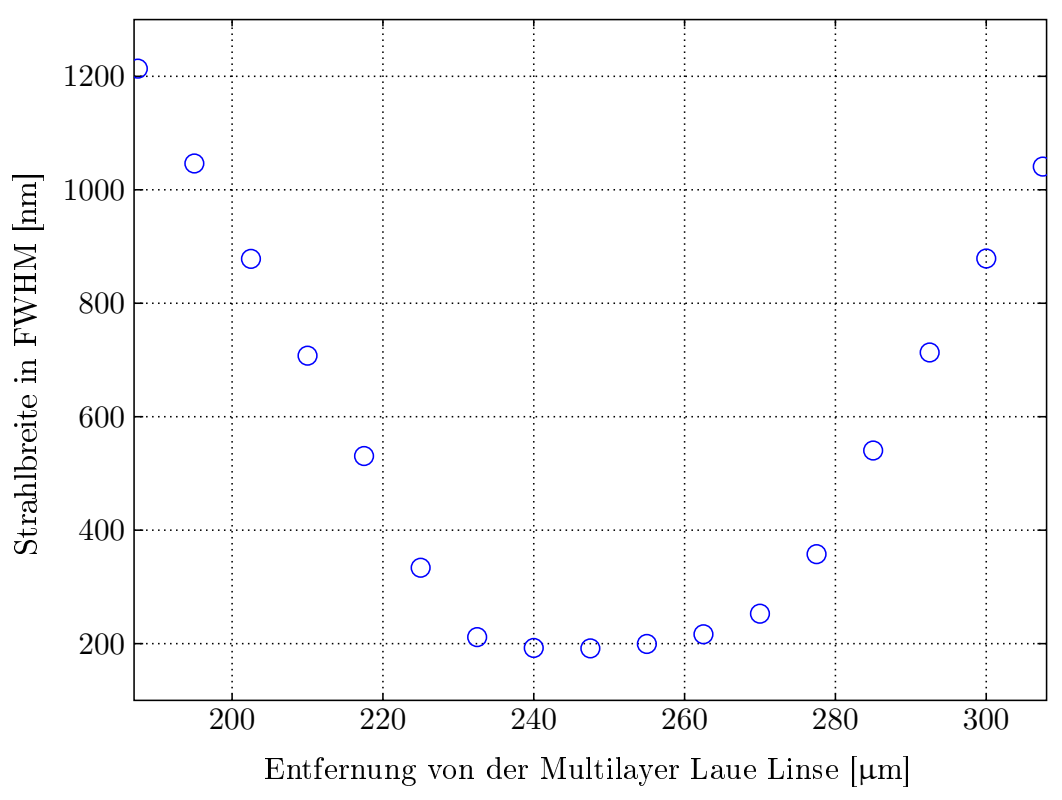

Abbildung 3.32:

Auswertung der Strahlbreiten der fokussierenden Beugungsordnung hinter Probe 1b. An ihrer engsten Stelle misst sie $182 \mathrm{~nm}$.

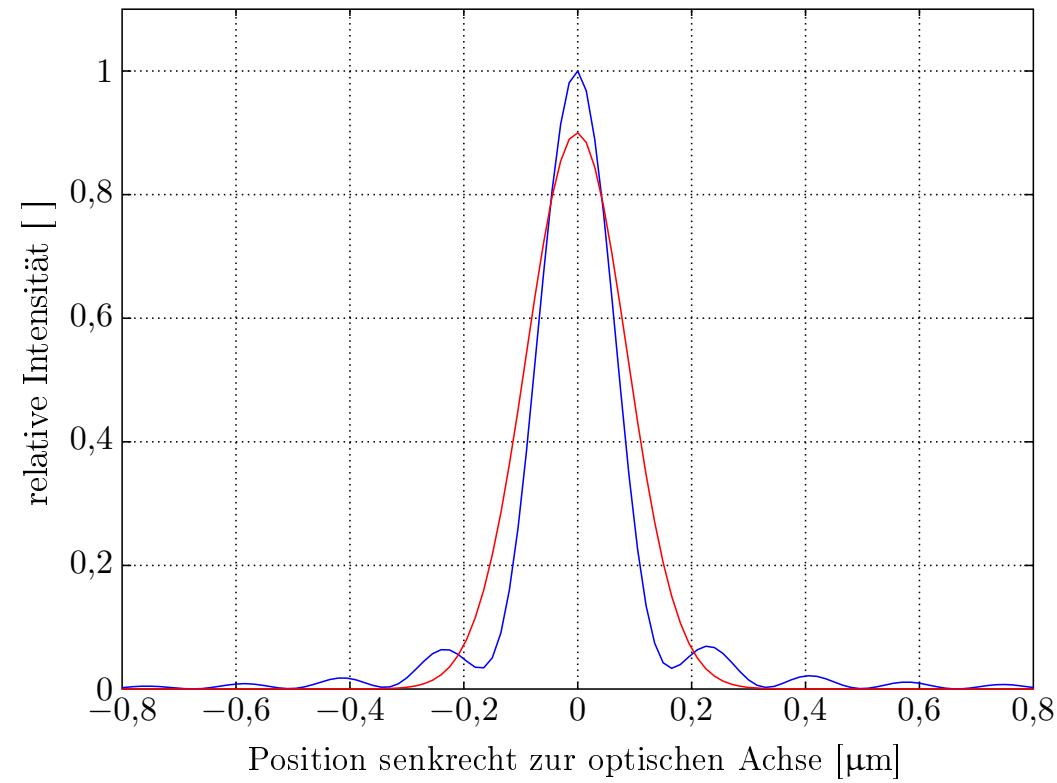

Abbildung 3.33:

Simulation der Intensität in Bildebene von Multischicht-Laue-Linse 1b. Aus der Intensitätsverteilung (blau) werden die Bildmomente berechnet. Die Halbwertsbreite der zu diesen Parametern assoziierten Normalverteilung (rot) beträgt $182 \mathrm{~nm}$. 


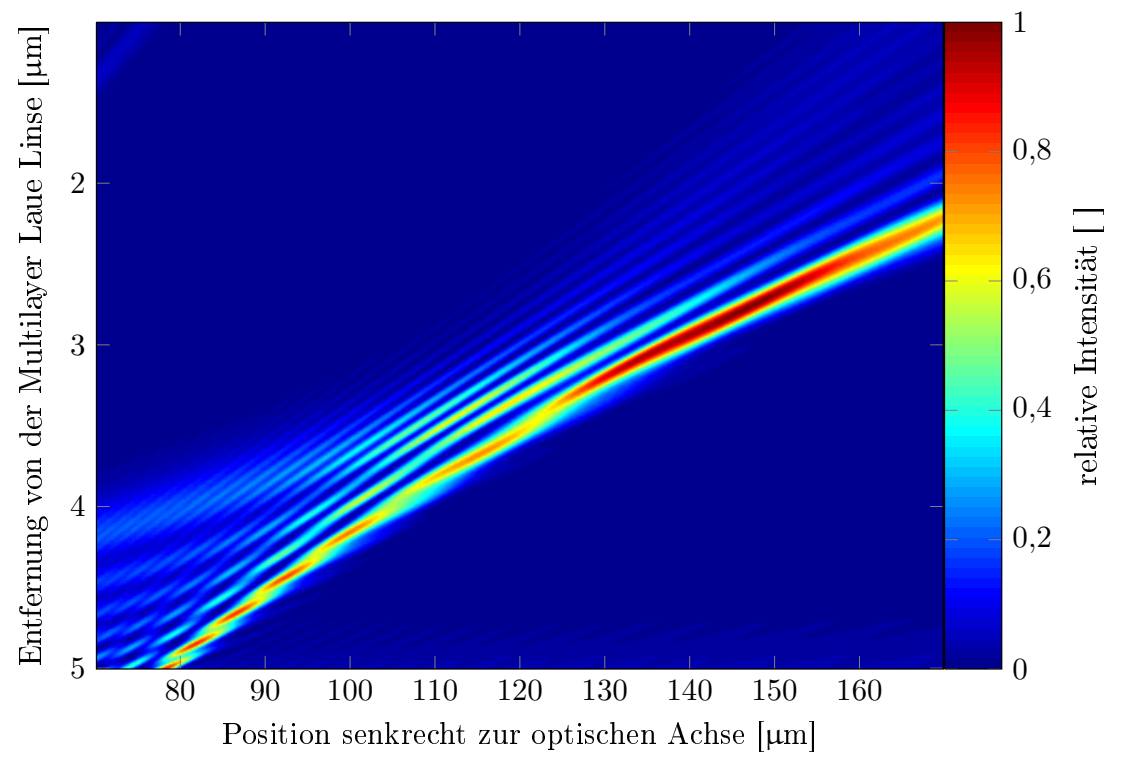

Abbildung 3.34:

Intensitätsverteilung des Wellenfelds um die Strahltaille der fehlerbehaftet simulierten Multischicht-LaueLinse 1b. Die Strahltaille zeigt ein Gaußstrahl untypisches Profil und erscheint aberriert. Die Position der geringsten Strahltaillenbreite nach Momentenmethode liegt bei etwa $123 \mu \mathrm{m}$ hinter der Multischicht-LaueLinse und beträgt $456 \mathrm{~nm}$.

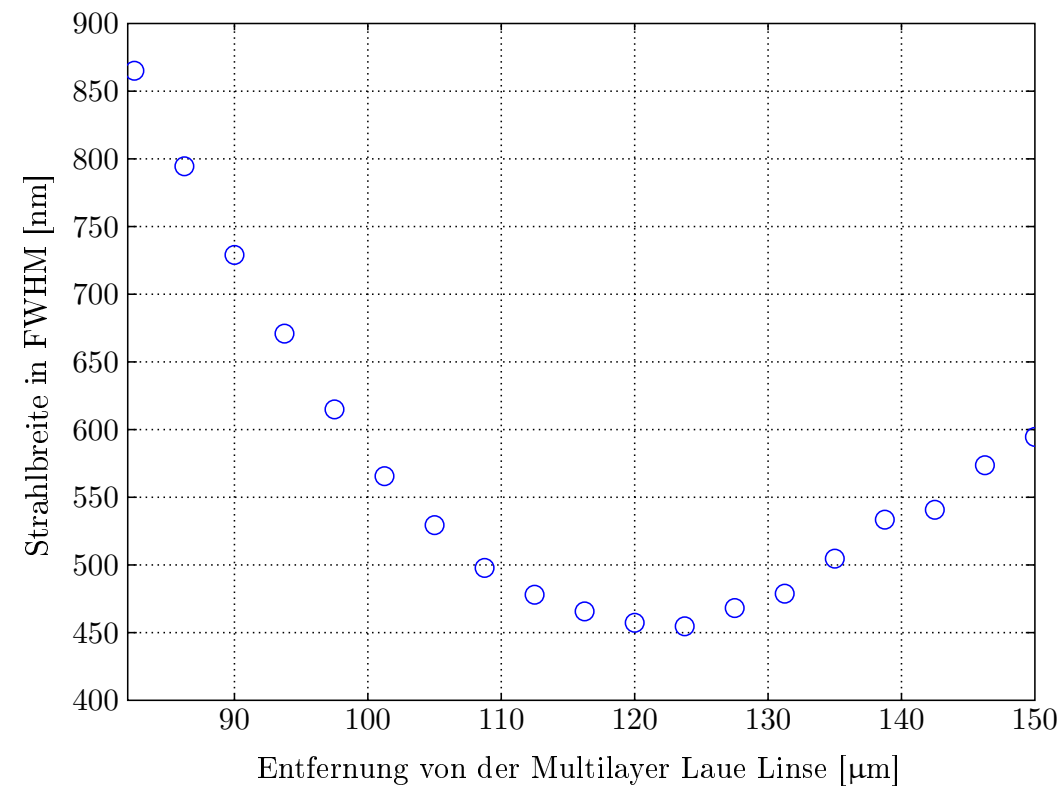

Abbildung 3.35:

Breite des Strahls hinter einer fehlerbehafteten Multischicht-Laue-Linse 1b. Die Strahlbreite wurde an verschiedenen Ebenen hinter der Multischicht-Laue-Linse berechnet. Dabei zeigt sich die stärkste Verjüngung des Strahls $456 \mathrm{~nm}$ hinter der Probe 1. 


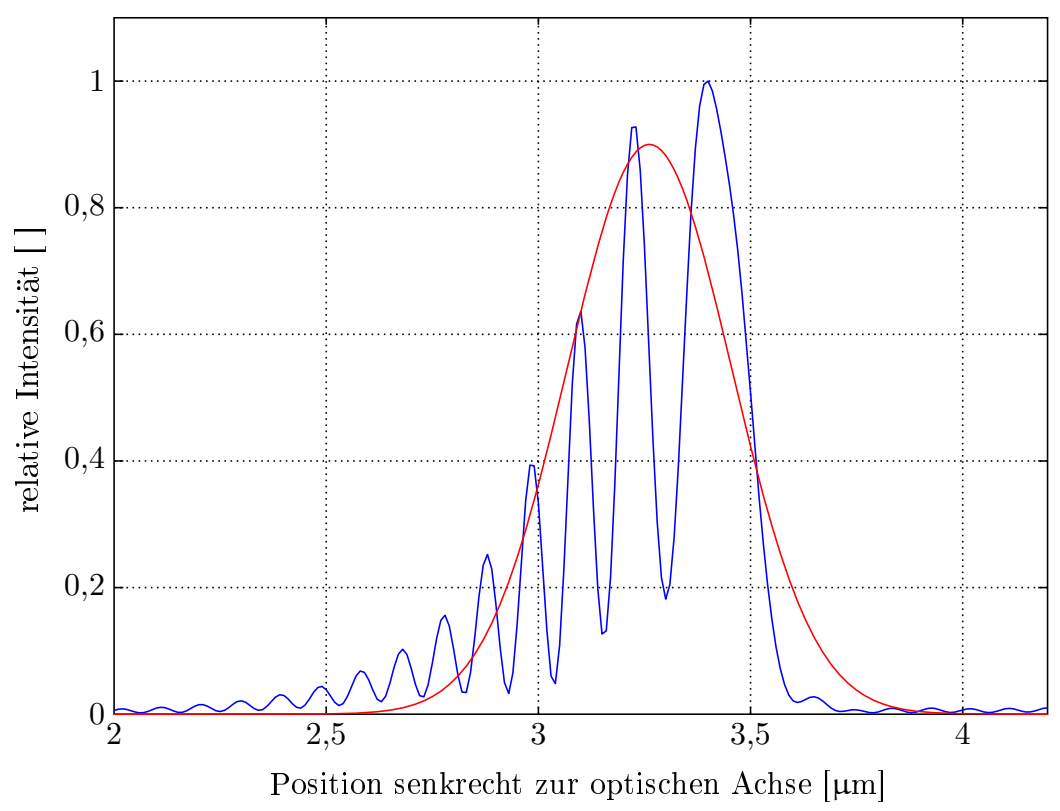

Abbildung 3.36:

Strahltaille der fehlerbehaftet simulierten Multischicht-Laue-Linse 1b. In der Strahltaille (blau) zeigt sich eine ausgeprägte Feinstruktur, die der experimentell beobachteten ähnelt. Die Halbwertsbreite der aus den Bildmomenten bestimmten Normalverteilungskurve (rot) beträgt $396 \mathrm{~nm}$.

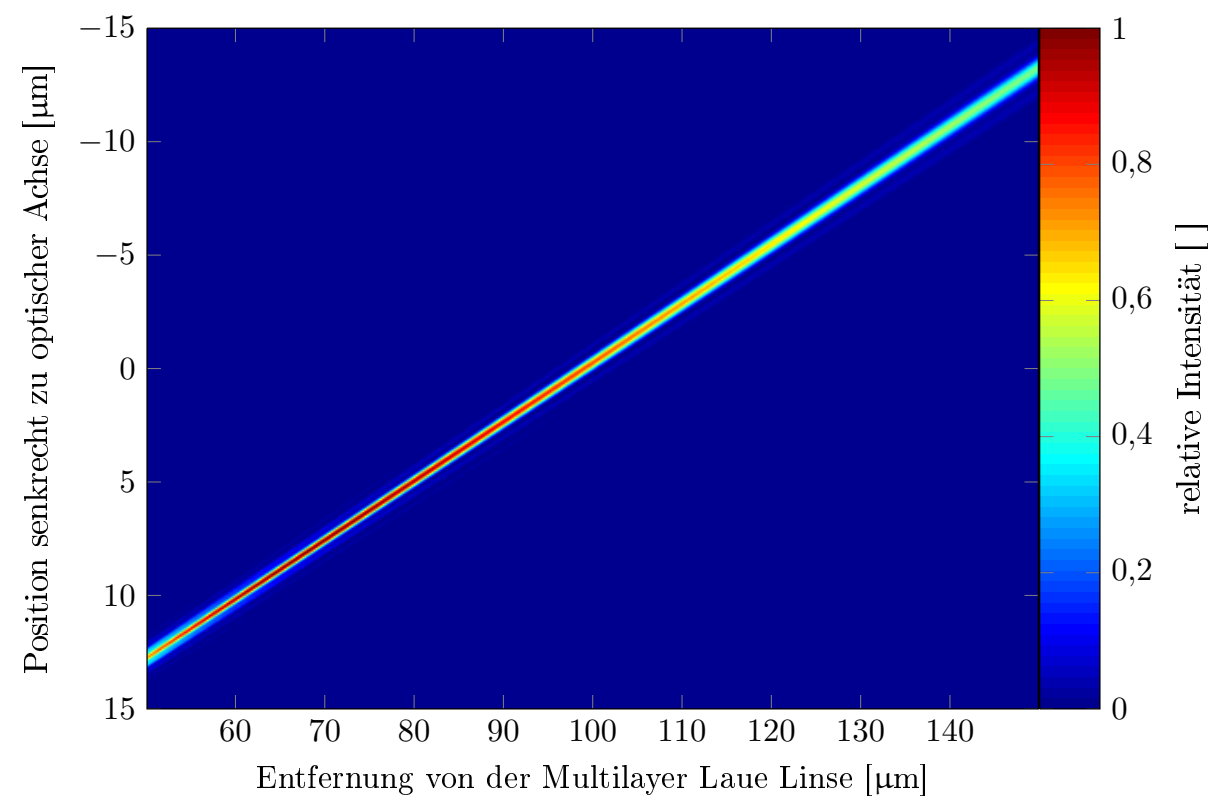

Abbildung 3.37:

Auftragung der Intensitäten des Wellenfelds hinter der Multischicht-Laue-Linse 2. Aufgrund der kleinen Apertur ist keine deutliche Strahltaille zu erkennen. 


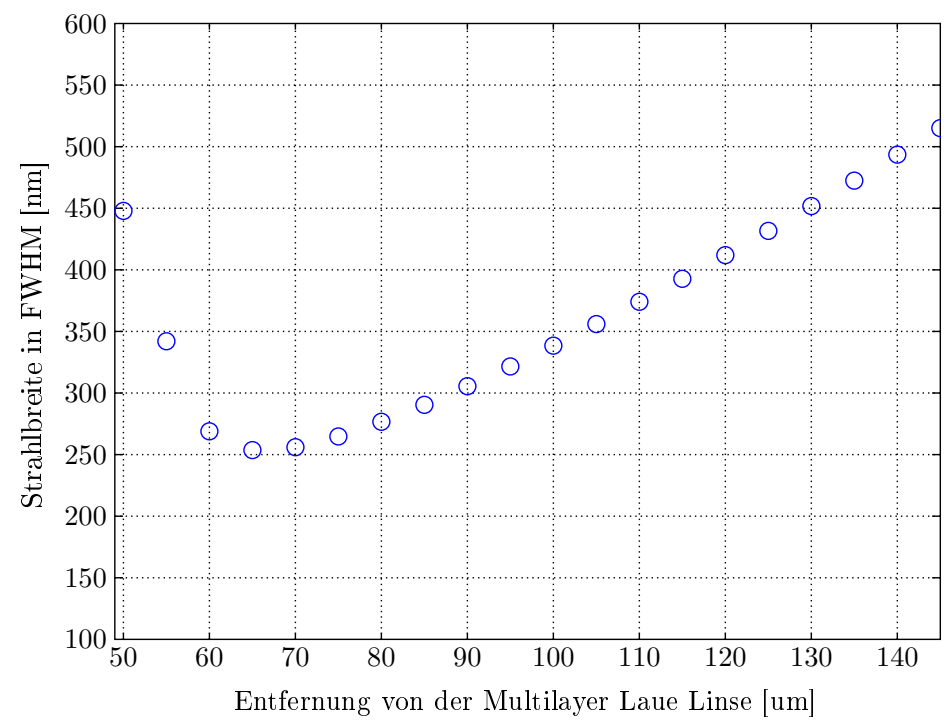

Abbildung 3.38:

Strahlbreiten der fokussierenden Beugungsordnung bei Multischicht-Laue-Linse 2. Durch die kleine Apertur und die geringe Zonenanzahl findet sich die Stelle der stärksten Verjüngung des Strahls vor dem vorgesehenem Bildpunkt. Eine Abschätzung der mittleren Intensität in der Zonenplattenebene und der maximalen Intensität im Fokus zeigt eine Intensitätsverstärkung von 1 zu 8. Die minimale Strahltaille, bestimmt durch Momentenmethode, beträgt bei einem Abstand von $66 \mu \mathrm{m}$ hinter der Probe $253 \mathrm{~nm}$. In der designten Bildebene von $100 \mu \mathrm{m}$ sind es $308 \mathrm{~nm}$.

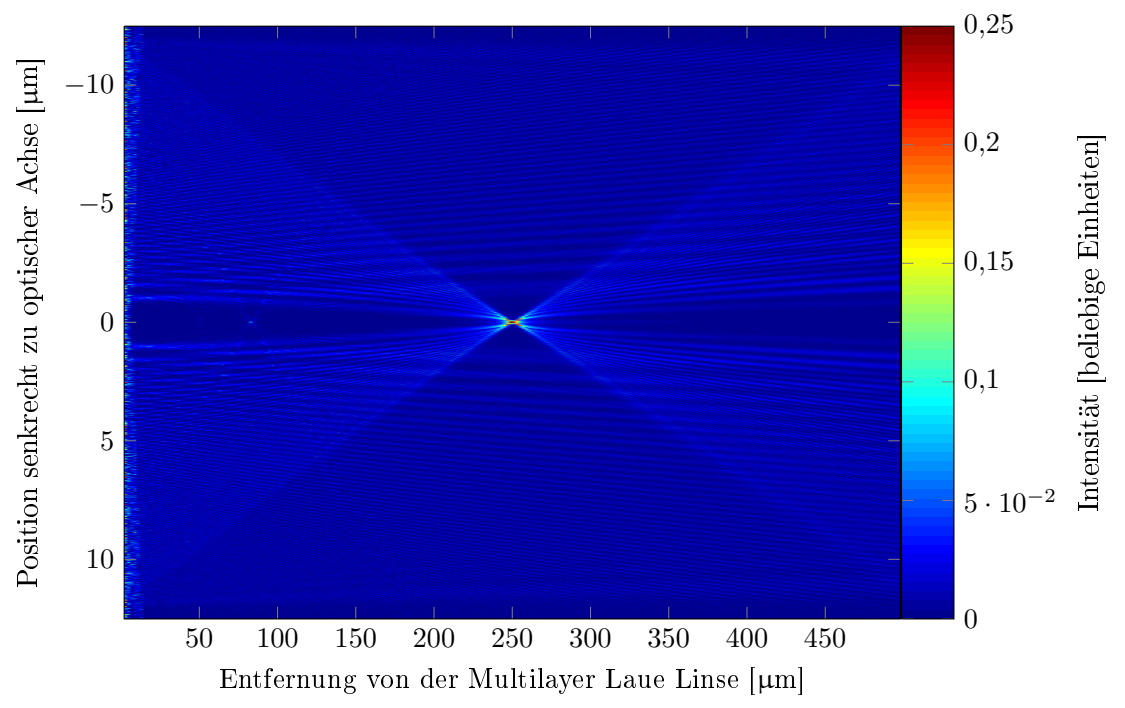

Abbildung 3.39:

Simulation des Wellenfelds hinter einer Vollfeld Multischicht-Laue-Linse mit 200 Zonen über eine Realisation. Die Beugungsordnungen der Schichten fallen auf einem gemeinsamen Bildpunkt zusammen. Eine Abschätzung der mittleren Intensität in der Zonenplattenebene und der maximalen Intensität im Fokus zeigt eine Intensitätsverstärkung von 1 zu 15 . Die relative Intensität ist so hoch, dass, damit verglichen, die transmittierte 0 . Beugungsordnung nur noch zu einem geringen Untergrund beiträgt. 


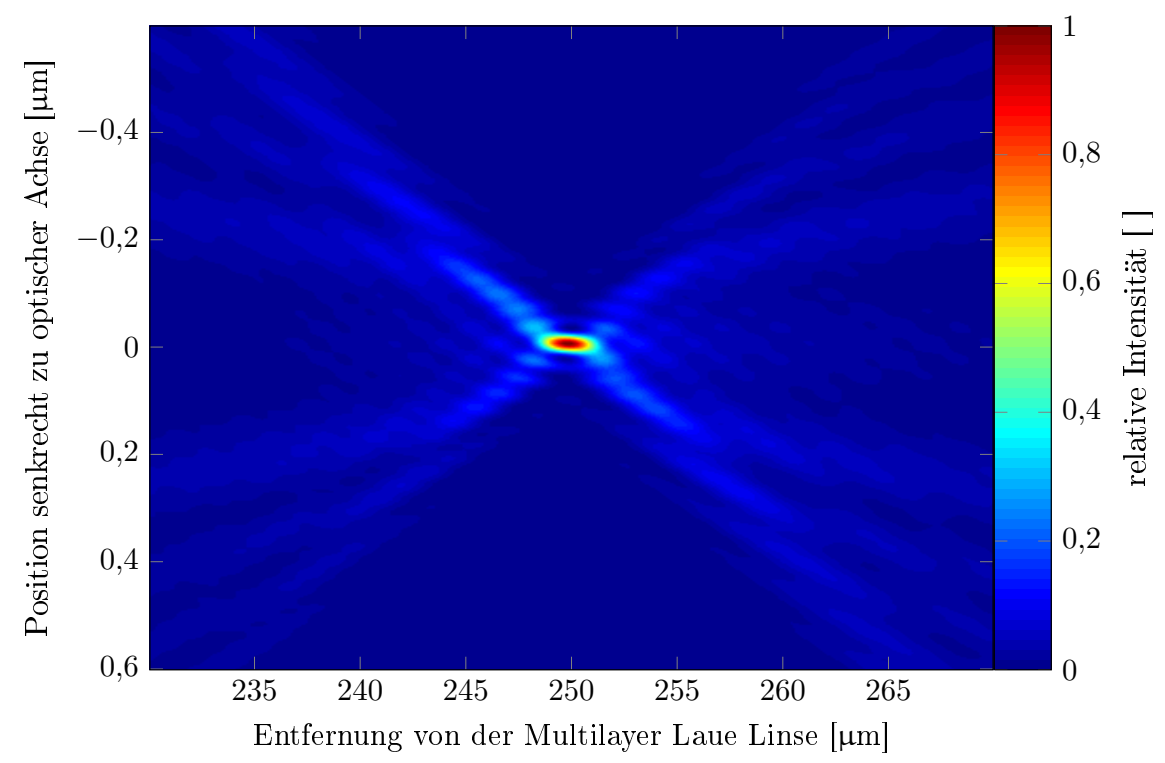

Abbildung 3.40:

Detailsimulation des Bildpunkts des Wellenfelds aus Abbildung 3.39 über 2500 Realisationen.

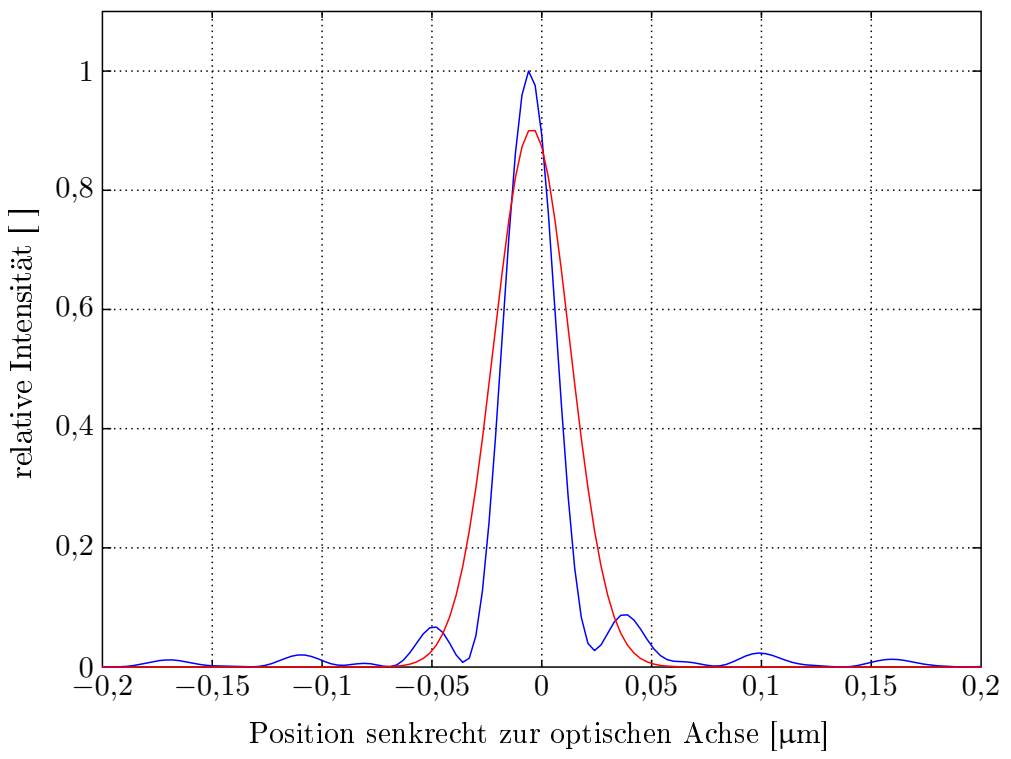

Abbildung 3.41:

Intensitätsverteilung (blau) in der Bildebene einer Vollfeld Multischicht-Laue-Linse mit 200 Zonen. Der zu denen aus der Momentenmethode gewonnenen Strahlparametern gehörige Gaußstrahl ist zur Anschauung mit aufgetragen (rot) und hat eine Halbwertsbreite von $40 \mathrm{~nm}$. 


\subsection{Vergleich und Diskussion der Ergebnisse}

Abschließend soll ein Überblick über die gewonnenen Ergebnisse gegeben werden. Ferner werden bis jetzt noch nicht erwogene Fehlerquellen diskutiert.

\subsubsection{Multischicht-Laue-Linse 1a/b}

Die Simulationen geben einen guten Aufschluss über das optische Verhalten der Multischicht-Laue-Linse im Experiment. Das doppelte Schichtsystem von Probe 1 ist dabei qualitativ und quantitativ gut beschrieben und verstanden. Die Abbildungsleistung der Einzelschicht von $375 \pm 30 \mathrm{~nm}$ Strahltaillenbreite bleibt hinter den Erwartungen aus den Simulationen zurück. Dies liegt im Wesentlichen an einem Fehler der Schichtdicken bei der Herstellung der Probe. Ihr im Experiment beobachtetes optisches Verhalten, nämlich eine starke Feinstrukturierung des Strahls und keine gemeinsame Strahltaille der beiden Schichtpakete kann durch numerische Simulation näherungsweise beschrieben werden. Der Ansatz eines linear zunehmenden Schichtdickenfehlers hat sich als mit der Beobachtung im Experiment und Inspektion unter dem Rasterelektronenmikroskop übereinstimmend herausgestellt. Die Schichten der Multischicht-Laue-Linse werden in Auftragungsrichtung zunehmend dünner als die Sollwerte. Daher ist die beobachtete Verkürzung der Bildpunktweite und des größer werdenden Divergenzwinkels nicht überraschend. Die Abschätzung der Brennweite und des Fokusdurchmessers allein aus der Verbreiterung des fokussierenden Beugungsmaximums - des Divergenzwinkels also ist, vor dem Hintergrund der beobachteten und simulierten Aberration, problematisch. Erst ein Vergleich der Divergenz von 0. zu der fokussierenden Beugungsordnung liefert eine verlässlichere Abschätzung für die mittlere Brennweite der Probe. Aufschluss auf die tatsächliche Breite der Strahltaille kann nur aus der Schneidblendenrastermessung zuverlässig gewonnen werden.

Ein Vergleich der integrierten Intensitäten der fokussierenden zur 0. Beugungsordnung zeigt ein Verhältnis von 3 zu 10. Das ist nur $25 \%$ weniger als die theoretisch maximale Effizienz einer Absorptionszonenplatte (siehe [Attwood, 1999]).

\subsubsection{Multischicht-Laue-Linse 2}

Da durch die geringe Effizienz der Probe nur wenige Messdaten erhoben werden konnten, ist das Verhalten von Probe 2 nur eingeschränkt zu erklären. Die Simulation beschreibt mit den simulierten Parametern nicht, aus welchem Grund Probe 2 kein für eine Divergenzwinkelmessung oder eine Schneidblendenrastermessung ausreichend intensives Signal liefert. Lediglich die Brennweite der Multischicht-Laue-Linse konnte aus der Lage des 0 . zum 1. Beugungsmaximums bestimmt werden. 
Mögliche Ursachen oder Beiträge dieses Problems sind nicht in der Simulation erfasst. Möglich ist die, eventuell fehlerbehaftete, endliche Dicke der Multischicht-Laue-Linse, die keinen ausreichenden Kontrast zwischen den Zonen bietet. Auch eine Verdrillung des Schichtpakets beim Einbetten in die Aperturblende wurde beobachtet und ist eine mögliche Ursache für die Abnahme der Effizienz der Zonenplatte.

\subsubsection{Einfluss experimenteller Fehlerquellen}

Im Folgenden sollen mögliche experimentelle Fehlerquellen untersucht werden, die bei der Vermessung der Optiken aufgetreten sein können.

Der Draht, der als Schneidblende verwendet wird, könnte einen nicht über die Länge der Multischicht-Laue-Linse konstanten Durchmesser und Oberfläche haben. Er wurde vor dem Einsatz daher mikroskopisch untersucht. Es konnten keine Störungen an Durchmesser oder Oberfläche sowie durch Montage auf den Halter identifiziert werden. Eine Abschätzung der maximalen Störeinflüsse des Drahts liegt bei wenigen $10 \mathrm{~nm}$.

Relevanter ist die genaue Ausrichtung der Zonenplatte zu der Schneidblende. Diese wird mittels Markierungen auf der Aperturblende vorjustiert und später im Experiment durch Vergleich der Beugungsrichtung der Multischicht-Laue-Linse und des Schattenwurfs von Resten der durch die Aperturblende transmittierten Strahlung überprüft. Die Ausrichtung lässt sich dabei auf einen Pixel über den Dünnungsbereich des $\mathrm{Si}_{3} \mathrm{~N}_{4}$-Membran von $1,5 \mathrm{~mm}$ genau bestimmen, sodass es über die Breite der Optik zu einer maximalen Dejustage von bis zu $100 \mathrm{~nm}$ kommen kann.

Vibrationen, verursacht durch die notwendigen Vakuumpumpen, erschüttern den Aufbau. Ihr Einfluss auf das Experiment wird mit ebenfalls bis zu $100 \mathrm{~nm}$ abgeschätzt.

Eine Verkippung der Linsen um bis zu $4^{\circ}$ wurde in der Simulation nachgestellt, konnte aber bei dem Auflösungsvermögen der vorhandenen Proben nicht als Fehlerquelle identifiziert werden. Die simulierten Bildpunktbreiten sind in Tabelle 3.7 zusammengefasst.

Die piezogetriebenen Lineartische wurden ferner mittels eines Vibrometers (SIOS SP-S 120) auf ihre Stellgenauigkeit untersucht. Da die Motoren während einer Belichtung abgeschaltet sind, verursachen sie keine zusätzlichen Bewegungen während der Messung. Ihre reproduzierbare Stellgenauigkeit lag im Bereich der Herstellerangabe von $50 \mathrm{~nm}$. Allerdings zeigte sich, dass Temperaturschwankungen die Schneidblende um bis zu $1 \frac{\mu \mathrm{m}}{\mathrm{K}}$ bewegen können. Dieses wurde als stärkste Fehlerquelle identifiziert und der Versuch daher unter klimatisierten Bedingungen und längeren Akklimatisierungszeiten nach Belüften des Aufbaus oder häufigen und weiten Verfahren der Piezomotoren durchgeführt. Beinahe alle diskutierten experimentellen Fehler des Schneidblenden-Aufbaus würden zu einer zu großen Strahlbreite führen. Das Messergebnis wird daher als obere Grenze der Strahltaille angesehen. 


\subsubsection{Fazit}

Das Simulationsprogramm geht von transmittiven und opaken Schichten aus. Noch berücksichtigt es nicht das Kontrast- und Phasenverhältnis der aus der Multischicht-LaueLinse austretenden Strahlung. Auch ein mögliches Beeinflussen der Felder innerhalb der absorptiven- und transmittiven Zonen wird nicht berücksichtigt [Kopylov u. a., 1995]. Das Aspektverhälnis der einzelnen Zonen ist dabei vergleichbar mit den ultra-kleinen Blenden, deren Eigenschaften im folgenden Kapitel 4 untersucht werden. Dennoch werden die experimentellen Beobachtungen in guter Näherung wiedergegeben.

Die Simulationen und Messungen sowie die Fehlerbetrachtungen des Experiments geben eine gute Beschreibung der Ergebnisse für Probe 1. Die Abweichung vom minimal zu erwartenden Fokus kann durch eine Kombination von Herstellungsfehlern, die durch Aberration in Strahltaillennähe und Detektorebene offenbar werden sowie durch experimentelle Störquellen erklärt werden. Die Aberrationen sind in der Simulation gut beschrieben, wobei die Annahme des linearen Schichtfehlers nur eine Näherung ist. Die meisten experimentellen Fehler können nur abgeschätzt werden, sodass eine Trennung in die Beiträge, die die einzelnen Fehler leisten, nicht möglich ist.

Probe 2 konnte aufgrund der geringen Effizienz experimentell nur wenig charakterisiert werden. Die beobachteten Eigenschaften entsprechen jedoch den Erwartungen und Simulationen.

Um das angestrebte Ziel einen Bildpunkt von unter $100 \mathrm{~nm}$ Breite an einer Laborröntgenquelle im Wasserfenster zu erreichen, wurde ein geeignetes Experiment simuliert und die prinzipielle Machbarkeit durch die durchgeführten Experimente demonstriert. 
Tabelle 3.7: Aus Simulation und Messungen gewonnene minimale Strahltaillenbreiten

\begin{tabular}{|c|c|c|c|}
\hline \multirow{3}{*}{$\begin{array}{l}\text { Quellabstand kohärente Punkt- } \\
\text { quelle, Ganzlinse }\end{array}$} & \multicolumn{3}{|c|}{ minimale Strahltaillenbreite } \\
\hline & $40 \mathrm{~cm}$ & $80 \mathrm{~cm}$ & $120 \mathrm{~cm}$ \\
\hline & $128,3 \mathrm{~nm}$ & $128,3 \mathrm{~nm}$ & $128,4 \mathrm{~nm}$ \\
\hline \multirow{2}{*}{$\begin{array}{l}\text { Quellabstand ausgedehnte, inko- } \\
\text { härente Quelle, Ganzlinse }\end{array}$} & $40 \mathrm{~cm}$ & $80 \mathrm{~cm}$ & $120 \mathrm{~cm}$ \\
\hline & $171,7 \mathrm{~nm}$ & $139,8 \mathrm{~nm}$ & $131,9 \mathrm{~nm}$ \\
\hline \multirow{2}{*}{ Verkippung, Ganzlinse } & $0^{\circ}$ & $2^{\circ}$ & $4^{\circ}$ \\
\hline & $139,8 \mathrm{~nm}$ & $139,4 \mathrm{~nm}$ & $139,6 \mathrm{~nm}$ \\
\hline $\begin{array}{l}\text { linearer } \quad \text { Herstellungsfehler, } \\
\text { Ganzlinse }\end{array}$ & \multicolumn{3}{|c|}{$1700 \mathrm{~nm}$ bei $\mathrm{f}=240 \mu \mathrm{m}$} \\
\hline \multirow{2}{*}{ Verkippung, Halblinse } & $0^{\circ}$ & $2^{\circ}$ & $4^{\circ}$ \\
\hline & $182,0 \mathrm{~nm}$ & $181,8 \mathrm{~nm}$ & $181,4 \mathrm{~nm}$ \\
\hline $\begin{array}{l}\text { linearer Herstellungsfehler, Halb- } \\
\text { linse }\end{array}$ & \multicolumn{3}{|c|}{$456 \mathrm{~nm}$} \\
\hline \multirow{2}{*}{ Messwert, Halblinse } & \multicolumn{2}{|c|}{$\begin{array}{l}\text { Schneidblenden- } \\
\text { rastermessung }\end{array}$} & Divergenzwinkel \\
\hline & \multicolumn{2}{|c|}{$375 \pm 30 \mathrm{~nm}$} & $180 \mathrm{~nm}$ \\
\hline
\end{tabular}




\section{Kapitel 4}

\section{Ultra-kleine Lochblenden}

\section{Einleitung}

Weiche Röntgenstrahlung bietet bei der Untersuchung kleinster Strukturen von organischen aber auch anorganischen Proben eine Vielzahl experimenteller Ansätze wie Mikroskopie, Spektroskopie oder Lithografie. Zur Erzeugung hoher räumlicher Kohärenz und hoch definierter Beleuchtung von Proben kommen kleinste Aperturen zum Einsatz, die auch entscheidend das Auflösevermögen des Experiments bestimmen [Eisebitt u. a., 2004; Knaack u. a., 2004; Barth u. a., 2010; Lim u. a., 2011]. In diesem Abschnitt werden die genauen optischen Eigenschaften solcher Aperturen im Wellenlängenbereich des Wasserfensters durch Simulation und Experiment untersucht. Es zeigen sich dabei einsetzende wellenleitende Eigenschaften, die das klassisch erwartete Verhalten verändern, bei dem man eine planare Absorptionsmaske erwartet, deren transmittierter Teil unverändert bleibt. Durch die Propagation in einer im Raum relevant ausgedehnten Apertur werden Änderungen des Wellenfeldes erwartet, die auch die Charakteristik der transmittierten Strahlung im Fernfeld beeinflussen [Fuhse und Salditt, 2006b].

Darüber hinaus wird die Einsatzfähigkeit dieser ultra-kleinen Lochblenden in der kohärenten Bildgebung in einem Experiment der sogenannten Resonanzptychographie demonstriert.

\subsection{Herstellung}

Die Herstellung von ultra-kleinen Lochblenden (UNH, englisch: ultra-narrow holes nach Fuhse und Salditt [2006b] ultra-narrow slits) sollte in einem gut absorbierenden

\footnotetext{
${ }^{4}$ Die Herstellung der ultra-kleinen Lochblenden wurde an der Universität Göttingen durchgeführt, wobei Christian Denker und Volker Radisch die Gerätezeit an Bedampfungsanlage und fokussiertem Ionenstrahl betreuten. Die Lochblenden wurden am Laser-Laboratorium Göttingen e.V. und am BESSY II Speicherring in Zusammenarbeit mit der Arbeitsgruppe Rosenhahn der Universität Heidelberg charakterisiert und für ptychographische Röntgenmikroskopie verwendet. Die Rekonstruktionen wurden von Mike Beckers (Universität Heidelberg) angefertigt.

Im Rahmen dieser Zusammenarbeit entstand die Veröffentlichung: Beckers, Senkbeil, Gorniak, Reese, Giewekemeyer, Gleber, Salditt und Rosenhahn [2011].
} 
und reflektierenden Material erfolgen sowie das Material in dünnen Schichten aufzutragen und mit fokussiertem Ionenstrahl gut zu bearbeiten sein. Dazu wurde Gold gewählt, das über den untersuchten Wellenlängenbereich einen kritischen Winkel von stets mehr als $5^{\circ}$ und eine Absorptionslänge von stets weniger als $100 \mathrm{~nm}$ hat. Eine kurze Absorptionslänge ist entscheidend dafür, dass der beleuchtende Synchrotronstrahl ausreichend gedämpft wird, damit der CCD-Detektor nicht übersteuert. Ein hoher kritischer Winkel bewirkt neben einem hohen Aspektverhältnis das frühe Einsetzen wellenleitender Eigenschaften (siehe Kabitel 1.3.1). Zur Herstellung der in dieser Arbeit untersuchten ultra-kleinen Lochblenden wurde eine Kombination aus Bedampfung zum Auftragen der dünnen Schichten auf $\mathrm{Si}_{3} \mathrm{~N}_{4}$-Membranen und fokussiertem Ionenstrahl eingesetzt. Dieser ist auch in der Literatur ein häufiges Werkzeug zur Erzeugung solcher kleinen Strukturen. Barth u.a. [2010] strukturierten eine ultra-kleine Lochblenden mit einem Durchmesser von $205 \mathrm{~nm}$ zur Röntgenbildgebung in $700 \mathrm{~nm}$ starke Goldfolie. Sannomiya u. a. [2011] erzeugt Lochblenden mit einem Durchmesser von $150 \mathrm{~nm}$ in Masken zur Oberflächenplasmonenresonanzspektroskopie in $50 \mathrm{~nm}$ starker Goldfolie.

\subsubsection{Aufdampfen}

Zur Herstellung dünner Goldfolien auf $\mathrm{Si}_{3} \mathrm{~N}_{4}$-Membranen kam das PVD-Verfahren (englisch: physical vapour deposition - physikalische Gasabscheidung) des thermischen Verdampfens zum Einsatz. Dieses wird im Folgenden überblicksartig erläutert. Bei dem Herstellungsprozess wird das zu deponierende Material durch Erhitzen mit einer elektrischen Heizung in die Gasphase überführt. Es migriert unter Vakuumbedingungen durch eine geeignete geometrische Anordnung vorzugsweise in Richtung des zu beschichtenden Substrats, an dem es schließlich kondensiert. Die Methode eignet sich gut zum Übertrag großer Materialmengen, so dass auch Goldschichten von $1-2 \mu \mathrm{m}$ Stärke deponiert werden können. Nachteilig ist, dass im Allgemeinen und im Gegensatz zur pulsed laser deposition (Kapitel 3.1.2) kein stöchiometrisch konstanter Materialübertrag bei Materialgemischen erfolgt. Dies ist in dem hier durchgeführten Experiment, in dem reine Metalle übertragen werden, ohne Relevanz.

Goldschichten verschiedener Dicken wurden so in einer Bedampfungsanlage (Balzers BAE 250, 4. physikalisches Institut der Universität Göttingen) auf $\mathrm{Si}_{3} \mathrm{~N}_{4}$-Membrane (Silson Ltd.) aufgebracht. Diese Substrate besitzen einen gedünnten Bereich von lediglich $100 \mathrm{~nm}$ Stärke, so dass die aufgetragene Schicht als Folie betrachtet werden kann. Als Haftvermittler wurde eine $3 \mathrm{~nm}$ starke Schicht Chrom eingesetzt, die eine gute Bindung und Abriebfestigkeit zwischen Substrat und Goldschicht sicherstellt. Die Schichtdicke wurde während der insgesamt sechs Depositionsvorgänge mit einem Schwingquarz in situ überprüft und anschließend mit einem Profilometer (Veeco DekTak 6M) genau bestimmt. Eines der gemessenen Oberflächenprofile ist in Abbildung 4.1 dargestellt. Die 


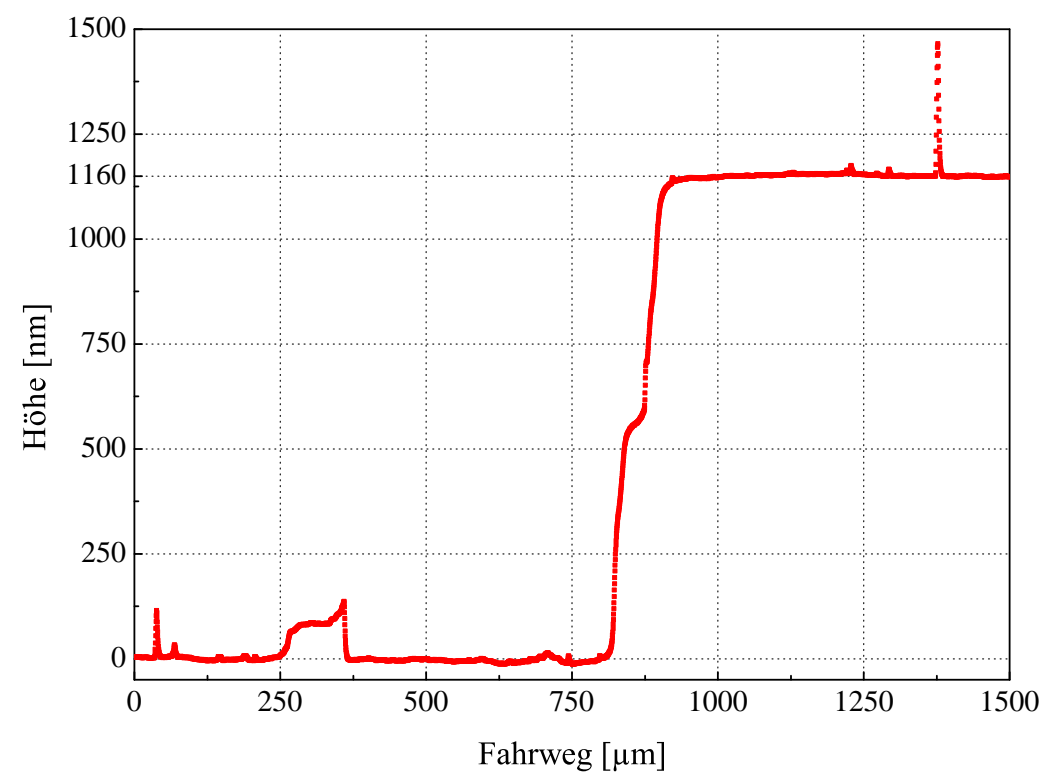

Abbildung 4.1:

Messung des Oberflächenprofils einer Goldfolie. Der Sprung von $1160 \mathrm{~nm}$ in der Profilhöhe ist zwischen der nicht beschichteten Rahmen der $\mathrm{Si}_{3} \mathrm{~N}_{4}$-Membran und der aufgetragenen Goldschicht und gibt daher ihre Dicke an.

für die Herstellung der ultra-kleinen Lochblenden verwendeten Folien messen 550, 980, 1160, 1470 und $1860 \mathrm{~nm}$ Dicke.

\subsection{2 fokussierter lonenstrahl}

Ein Überblick über die Funktionsweise des fokussierten Ionenstrahls wird in Kapitel 3.1.3 gegeben. Die mit Gold beschichteten $\mathrm{Si}_{3} \mathrm{~N}_{4}$-Membranen wurden mit dem Ionenstrahl von der Membranseite aus bestrahlt. Eine Reihe rasterelektronenmikroskopischer Aufnahmen zur Illustrierung der Lochblenden und des Herstellungsprozesses findet sich in Abbildung 4.2. Dabei wird bei kleinen Lochblenden erst auf einem großen Areal von $2 \mu \mathrm{m}$ die $\mathrm{Si}_{3} \mathrm{~N}_{4}$-Schicht abgetragen (30 pA Ionenstrom, 76 s Strahlzeit, 0,1 $\mu$ s Dwell Time, siehe Abbildung 4.2(b)). Anschließend wird ein zentrales Loch bei einem Ionenstrom von 10 pA gebohrt. Bei den größeren und tieferen Lochblenden wird bei hohem Ionenstrom (0,3 nA) ein Loch gebohrt, das circa 90\% des angestrebten Durchmessers besitzt. Danach wird die ultra-kleine Lochblende bei einem geringeren Ionenstrom bis zum gewünschten Radius erweitert. So kann eine Redeposition (Abbildung 4.2(c)) von abgetragenem Material weitgehend unterdrückt werden. Nicht jede Bohrung - insbesondere solche mit hohem Aspektverhältnis - war erfolgreich. Durch die Inspektion der Rückseite mit dem Rasterelektronenmikroskop kann darüber Klarheit gewonnen werden. 


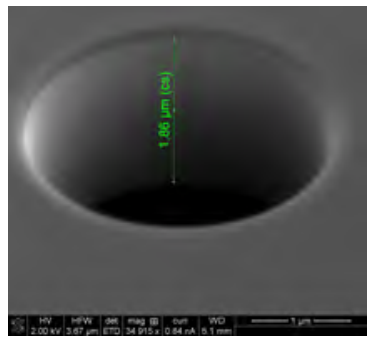

(a) $3 \mu \mathrm{m}$ Lochblende in $1,86 \mu \mathrm{m}$ starker Goldfolie (FEI Nova Nanolab, 2kV Spannung, 34915× Vergrößerung).

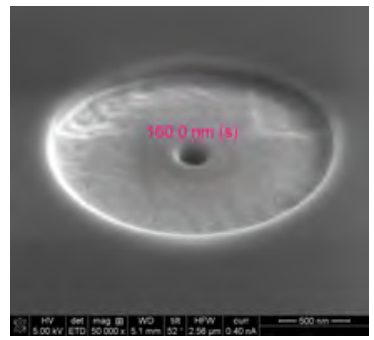

(b) $160 \mathrm{~nm}$ Lochblende in $550 \mathrm{~nm}$ Goldfolie. Im Bereich um das UNH ist auch das $\mathrm{Si}_{3} \mathrm{~N}_{4}$-Substrat abgetragen. (FEI Nova Nanolab, 5kV Spannung, $50000 \times$ Vergrößerung).

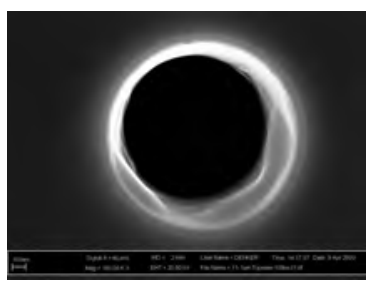

(c) In der Lochblende während der Strukturierung redeponiertes Material. (Zeiss LEO Supra 35, 20kV Spannung, 100000 $\times$ Vergrößerung) .

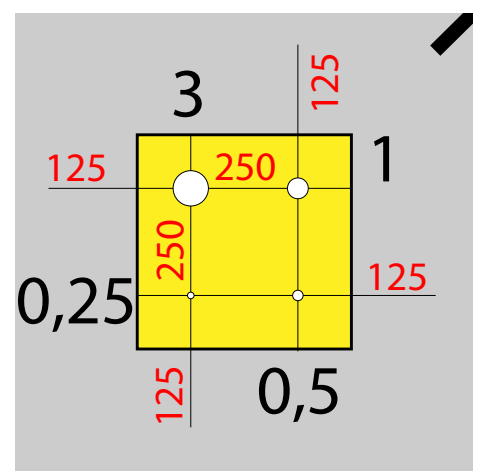

(d) Anordnung der Lochblenden bei der ersten Messzeit am BESSY II (Undulator UE52-SGM) in 1470 und $1860 \mathrm{~nm}$ starker Folie.

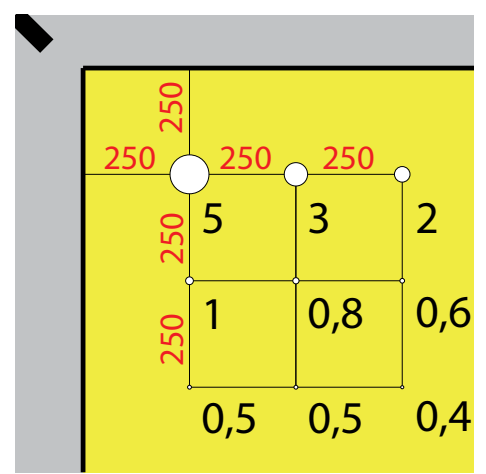

(e) Anordnung der Lochblenden bei der zweiten Messzeit am BESSY II (Undulator U49/2-PGM1) in $1860 \mathrm{~nm}$ starker Folie.

\section{Abbildung 4.2:}

Herstellung der ultra-kleinen Lochblenden. Dargestellt sind elektronenmikroskopische, exemplarische Bilder einer kleinen und einer großen Lochblende, strukturiert in Goldfolie. Die Anordnung der Lochblenden ist in (d) und (e) dargestellt, wobei die schwarzen Zahlen die Blendendurchmesser und die roten deren Abstände angeben. 


\subsection{Experimentelle Charakterisierung}

Um die ultra-kleinen Lochblenden optisch zu charakterisieren, wurden sie mit verschiedenen Quellen und Wellenlängen bestrahlt. Zunächst wurden Teststrukturen der Lochblenden an einer EUV-Laborquelle untersucht und dabei auf beginnende wellenleitende Eigenschaften getestet. Die anschließenden Experimente am BESSY II wurden mit verschiedenen Wellenlängen durchgeführt (Undulator UE52-SGM). Es wurden Beugungsbilder der Proben aufgenommen sowie ptychographische Mikroskopaufnahmen einer Wellenlänge angefertigt. Aus letzteren wird das Wellenfeld in Probenebene und durch Propagation auch am Blenden-Ausgang rekonstruiert und mit Simulationen der Wellenausbreitung in der Lochblende verglichen.

\subsubsection{Voruntersuchung der ultra-kleinen Lochblenden an einer EUV-Laborquelle}

Zur Voruntersuchung der ultra-kleinen Lochblenden wurden Proben verschiedener Aspektverhältnisse und Durchmesser mit einer Laborstrahlungsquelle bestrahlt, die in der Dissertationsschrift bei Barkusky [2010] ausführlich beschrieben ist. An dieser Stelle wird eine Zusammenfassung des Versuchsaufbaus und der verwendeten Komponenten gegeben. Zur Erzeugung von EUV-Strahlung kam bei dieser Laserplasmaquelle (Innolas Spitlight 600, ausgewählte Parameter in Tabelle 2.1 auf Seite 26) ein GoldFestkörpertarget zum Einsatz, das durch die größere Teilchendichte eine weitaus höhere Energiedichte als ein Gastarget ermöglicht. Das Targetmaterial ist dabei auf einem Zylinder aufgebracht, der nach jedem Laserpuls leicht gedreht wird, so dass das Plasma in neuem Material an gleicher Stelle zündet.

Um viel der in einem großem Raumwinkel abgestrahlten EUV-Strahlung zu nutzen, sammelt ein modifiziertes Schwarzschildobjektiv mit großer objektseitiger Apertur die Strahlung und bildet die Plasmaquelle stark in $530 \mathrm{~mm}$ Entfernung im Maßstab 10:1 verkleinert ab. Dabei wirken eine Zirkoniumfolie und die Vielschichtspiegel als spektrale Filter, so dass nur monochromatische Strahlung von 13,5 nm Wellenlänge den Belichtungspunkt von $3,8 \times 4,5 \mu^{2}(\mathrm{FWHM})$ Größe bei etwa $100 \mathrm{~nJ}$ Pulsenergie erreicht.

Die so erzeugte Strahlung traf als eine Hohlkegelbeleuchtung auf den LochblendenEingang, wobei die Strahlung unter einem Winkel von $12,7^{\circ}-26,6^{\circ}$ einfiel. Die die Lochblende passierende Strahlung wurde anschließend mit einer CCD-Kamera (Toshiba Teli CS8620, $768 \times 494$ Pixel, 8,4 × 9,8 $\mu^{2}$ ) mit Quantenkonverter (P43 Phosphorbeschichtung) unter einem Abstand von etwa $1 \mathrm{~mm}$ in Einzelpulsmessung detektiert. Der Versuchsaufbau ist in Abbildung 4.3 dargestellt. Eine Auswahl an aufgenommenen Bildern verschiedener Aspektverhältnisse ist in Abbildung 4.4 gegeben. Es zeigt sich, dass 


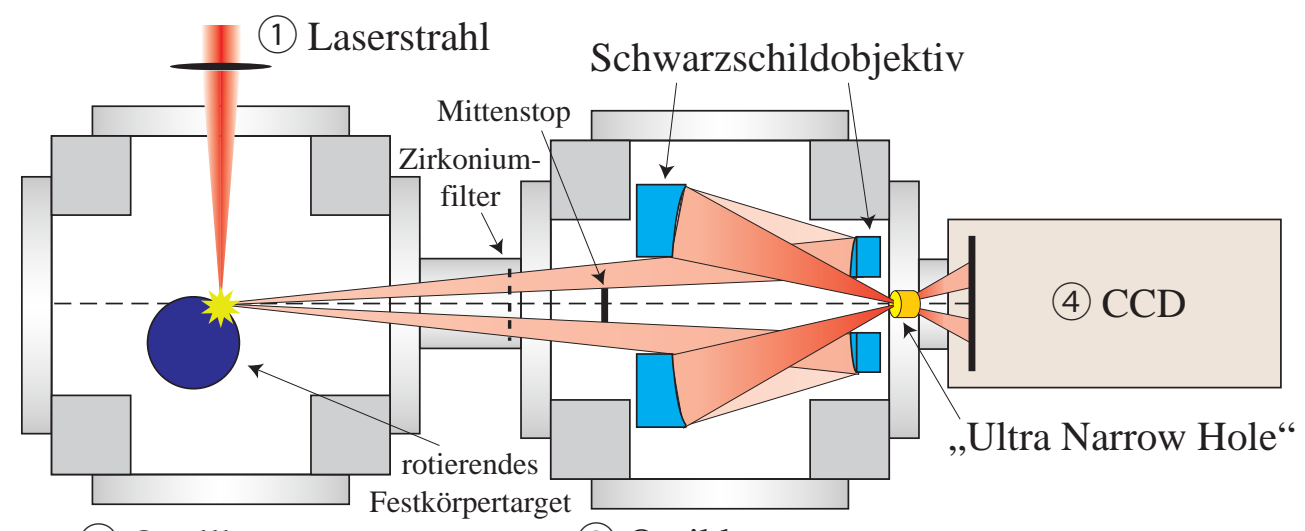

(2) Quellkammer (3) Optikkammer

\section{Abbildung 4.3:}

Der Aufbau des Experiments zur Voruntersuchung von ultra-kleinen Lochblenden. Der fokussierte Laserstrahl (1) zündet in der Quellkammer (2) im Gold-Festkörpertarget ein Plasma. Das aus diesem emittierte Licht wird durch eine Zirkonium-Folie in der Optikkammer gefiltert (3) und von einem modifizierten Schwarzschildobjektiv stark verkleinert aus dem Eingang der Lochblende abgebildet. Eine phosphorbeschichtete CCD-Kamera (4) registriert die durch sie transmittierte EUV-Strahlung.

die Hohlkegelbeleuchtung (Abbildung 4.4(a)) bei zunehmend kleineren und tieferen Löchern zunächst an der äußeren Apertur abgeschnitten wird. Bei noch weiter erhöhtem Aspektverhältnis wird auch die innere Apertur gefüllt und es zeigt sich schließlich ein beinahe homogenes Beleuchtungsniveau (Abbildungen 4.4(b) - 4.4(d)). Da immer weniger Strahlung in die kleiner werdenden Löcher eingekoppelt werden kann, ist die durch die Lochblende transmittierte Strahlung dann entsprechend gering. 


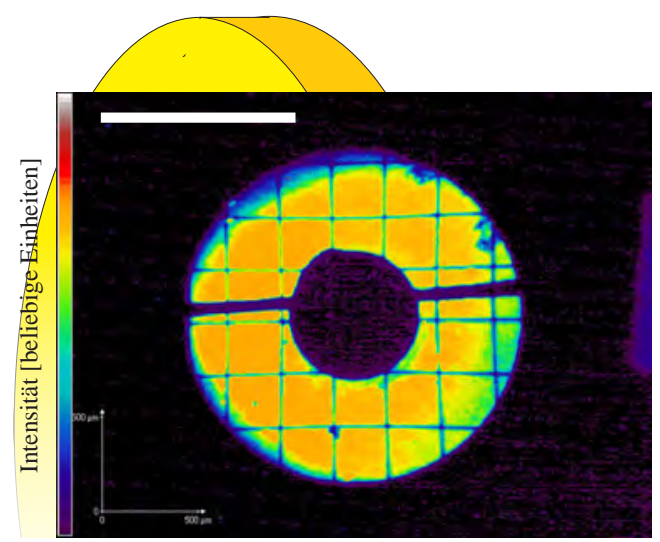

(a) Beleuchtungskegel hinter einer $5 \mu \mathrm{m}$ großen Lochblende in einer $550 \mathrm{~nm}$ starken Folie.

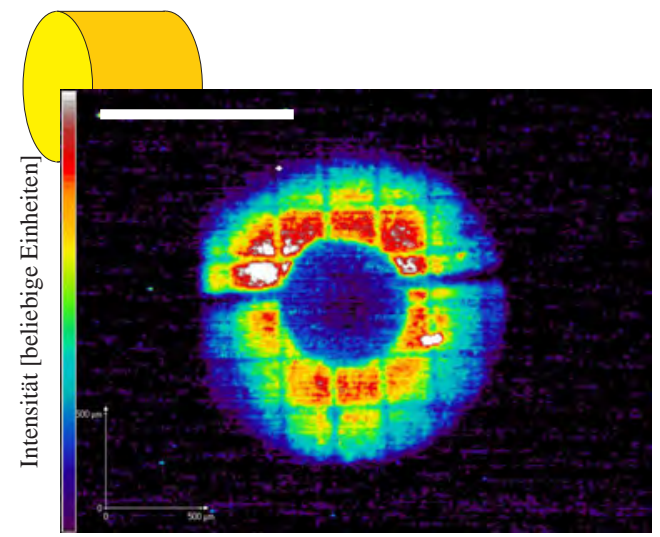

(c) Beleuchtungskegel hinter einer $1 \mu \mathrm{m}$ großen Lochblende in einer $1160 \mathrm{~nm}$ starken Folie. Die innere, eigentlich dunkle Apertur beginnt sich zu füllen.

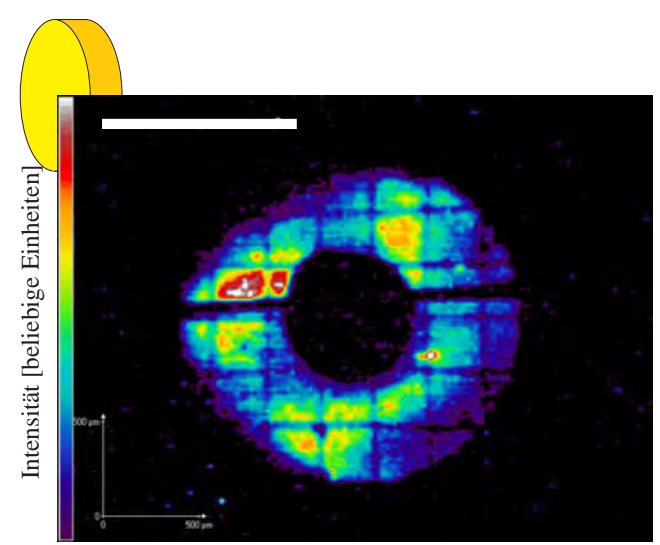

(b) Beleuchtungskegel hinter einer $1 \mu \mathrm{m}$ großen Lochblende in einer $550 \mathrm{~nm}$ starken Folie. Die äuBere Apertur wird leicht abgeschnitten.

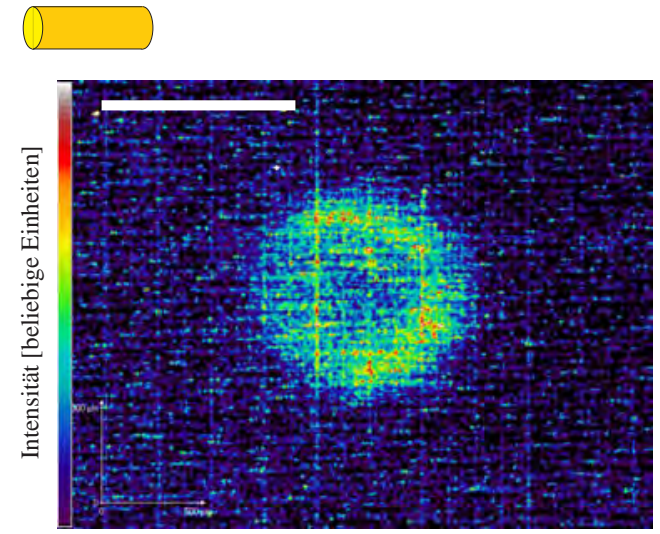

(d) Beleuchtungskegel hinter einer 0,4 $\mu \mathrm{m}$ großen Lochblende in einer $1160 \mathrm{~nm}$ starken Folie. Die äußere Apertur wird deutlich abgeschnitten und die innere Apertur füllt sich hingegen mit EUV-Strahlung.

\section{Abbildung 4.4:}

Bei den an der Laborquelle bestrahlten Proben zeigen sich mit zunehmendem Aspektverhältnis (illustriert durch gelbe Zylinder im Hintergrund) Phänomene, die mit Totalreflexion innerhalb der ultra-kleinen Lochblenden erklärt werden. Dies wird als einsetzende Wellenleitung interpretiert. Der weiße Balken in den Bildern zeigt eine Länge von $1 \mathrm{~mm}$ auf dem CCD-Chip an. 


\subsubsection{Wellenleitende Eigenschaften der ultra-kleinen Lochblenden}

Die Vorexperimente legen nahe, dass die ultra-kleinen Lochblenden Eigenschaften zeigen, die als wellenleitende Phänomene gedeutet werden können. Die Beugungserscheinungen hinter den Lochblenden sollen genauer untersucht werden. Wie theoretisch für planare Wellenleiter, den ultra-narrow slits durch Fuhse und Salditt [2006b] und Fuhse [2006] untersucht, wird das Beugungsbild auf Abweichungen von einer mathematischen Blende untersucht. Eine solche Lochblende ist unendlich dünn und das absorbierende Material tilgt die Strahlung vollständig. Die radialsymmetrische Intensitätsverteilung ihres Beugungsbildes ist durch

$$
I(r)=I_{0} \cdot\left(\frac{J_{1}(2 \pi r)}{\pi r}\right)^{2}
$$

gegeben. Dabei bezeichnet $I_{0}$ die eingestrahlte Intensität der Strahlung, $r$ den Abstand zur optischen Achse und $J_{1}$ ist die Besselfunktion erster Art. Eine ultra-kleine Lochblende ist hingegen von endlicher Tiefe, die das Beugungsverhalten der Lochblende beeinflusst. Eine Abschätzung für das Aspektverhältnis, bei dem die Mindesttiefe für eine Abweichung von der mathematischen Blende erreicht ist, kann durch einen kritischen Lochdurchmesser angegeben werden, bei der die gebrochene Welle die gegenüberliegende Seite der ultra-kleinen Lochblende trifft [Bergemann u. a., 2003; Fuhse und Salditt, 2006b]:

$$
d_{c}=l \cdot \tan \theta_{c}
$$

Dabei bezeichnet $l$ die Länge der ultra-kleinen Lochblende und $\theta_{c}$ den wellenlängenabhängigen kritischen Winkel der äußeren Totalreflexion, wie sie neben anderen Materialeigenschaften durch Henke u. a. [1993] tabelliert sind. In den Tabellen 4.1 und 4.2 werden diese Abschätzungen für die im Experiment verwendeten Proben angegeben. Darüber hinaus findet sich dort auch der dimensionslose Wellenleiterparameter $D$, der den Quotienten aus kritischer Weite $W=\lambda / 2 \cdot \theta_{c}$ und ultra-kleinen Lochblenden-Durchmesser angibt.

Diese Analyse zeigt bei einer Photonenenergie von $92 \mathrm{eV}$ eine starke Zunahme des kritischen Durchmessers $d_{c}$, der im Bereich der tatsächlich strukturierten Lochgrößen liegt. Für diese Energien erwartet man ein von der mathematischen Blende abweichendes optisches Verhalten. Zum Prüfen dieser Hypothese wurden die ultra-kleinen Lochblenden an der Synchrotronstrahlungsquelle BESSY II in Berlin bei verschiedenen Energien bestrahlt (Undulator UE52-SGM) und das Beugungsfeld in einem Abstand von 49,6 cm von einer rückseitig gedünnten CCD-Kamera aufgenommen (Andor Technology DX436, $2048^{2}$ Pixel, 13, $5^{2} \mu \mathrm{m}^{2}$ Pixelgröße). Die gewonnenen Detektorbilder zeigen hochsymme- 
Tabelle 4.1:

Parameter der in $1470 \mathrm{~nm}$ starker Folie gebohrten, ultra-kleinen Lochblende. Bei den jeweils verschiedenen Photonenenergien verändern sich die Materialparameter mitunter stark, so dass bei niedrigen Energien der kritische Durchmesser für das Eintreten von wellenleitenden Eigenschaften stark ansteigt.

\begin{tabular}{|c|c|c|c|c|c|c|c|}
\hline \multirow[t]{2}{*}{ Energie $E$} & \multirow{2}{*}{$\begin{array}{l}\text { Kritischer } \\
\text { Winkel } \theta_{c}\end{array}$} & \multirow[t]{2}{*}{$\beta / \delta$} & \multirow{2}{*}{$\begin{array}{l}\text { Kritische } \\
\text { Weite } W_{c}\end{array}$} & \multirow{2}{*}{$\begin{array}{l}\text { Kritischer } \\
\text { Durchmes- } \\
\text { ser } d_{c}\end{array}$} & \multicolumn{3}{|c|}{ Wellenleiterparameter $D$} \\
\hline & & & & & $\varnothing 3000 \mathrm{~nm}$ & $\varnothing 1023 \mathrm{~nm}$ & $\varnothing 437 \mathrm{~nm}$ \\
\hline $92 \mathrm{eV}$ & $25,7^{\circ}$ & 0,51 & $15,02 \mathrm{~nm}$ & $707 \mathrm{~nm}$ & 200 & 68 & 29 \\
\hline $260 \mathrm{eV}$ & $6,96^{\circ}$ & 1,44 & $19,63 \mathrm{~nm}$ & $179 \mathrm{~nm}$ & 153 & 52 & 22 \\
\hline $361 \mathrm{eV}$ & $6,36^{\circ}$ & 1,31 & $15,47 \mathrm{~nm}$ & $163 \mathrm{~nm}$ & 194 & 66 & 28 \\
\hline $500 \mathrm{eV}$ & $5,65^{\circ}$ & 0,98 & $12,57 \mathrm{~nm}$ & $145 \mathrm{~nm}$ & 239 & 81 & 35 \\
\hline
\end{tabular}

Tabelle 4.2:

Parameter der in 1860 nm starker Folie gebohrten, ultra-kleinen Lochblende.

\begin{tabular}{|c|c|c|c|c|c|c|c|}
\hline \multirow[t]{2}{*}{ Energie $E$} & \multirow{2}{*}{$\begin{array}{l}\text { Kritischer } \\
\text { Winkel } \theta_{c}\end{array}$} & \multirow[t]{2}{*}{$\beta / \delta$} & \multirow{2}{*}{$\begin{array}{l}\text { Kritische } \\
\text { Weite } W_{c}\end{array}$} & \multirow{2}{*}{$\begin{array}{l}\text { Kritischer } \\
\text { Durchmes- } \\
\text { ser } d_{c}\end{array}$} & \multicolumn{3}{|c|}{ Wellenleiterparameter $D$} \\
\hline & & & & & $\varnothing 3000 \mathrm{~nm}$ & $\varnothing 2040 \mathrm{~nm}$ & $\varnothing 905 \mathrm{~nm}$ \\
\hline $92 \mathrm{eV}$ & $25,7^{\circ}$ & 0,51 & $15,02 \mathrm{~nm}$ & $880 \mathrm{~nm}$ & 200 & 136 & 60 \\
\hline $260 \mathrm{eV}$ & $6,96^{\circ}$ & 1,44 & $19,63 \mathrm{~nm}$ & $223 \mathrm{~nm}$ & 153 & 104 & 46 \\
\hline $361 \mathrm{eV}$ & $6,36^{\circ}$ & 1,31 & $15,47 \mathrm{~nm}$ & $203 \mathrm{~nm}$ & 194 & 132 & 58 \\
\hline $500 \mathrm{eV}$ & $5,65^{\circ}$ & 0,98 & $12,57 \mathrm{~nm}$ & $181 \mathrm{~nm}$ & 239 & 162 & 72 \\
\hline
\end{tabular}




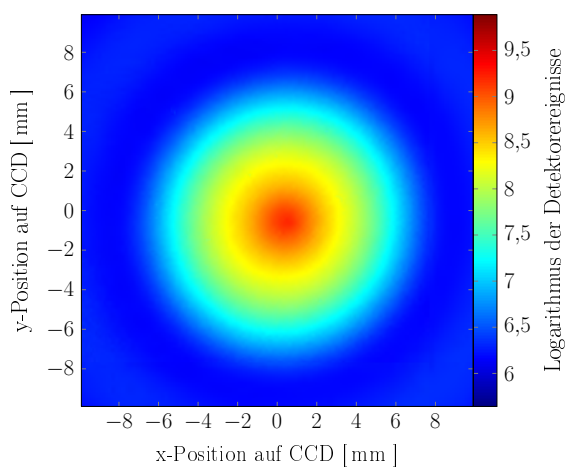

(a) Beugungsbild bei $92 \mathrm{eV}$ (Undulator UE52-SGM), bei $49,6 \mathrm{~cm}$ Abstand und $60 \mathrm{~s}$ Belichtungszeit.

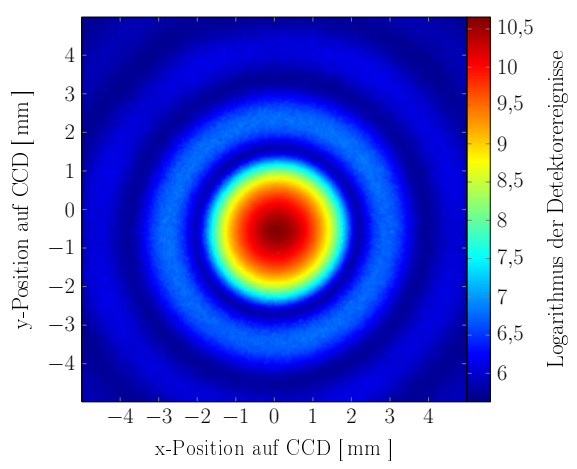

(c) Beugungsbild bei $361 \mathrm{eV}$ (Undulator UE52-SGM), bei $49,6 \mathrm{~cm}$ Abstand und $6 \mathrm{~s}$ Belichtungszeit.

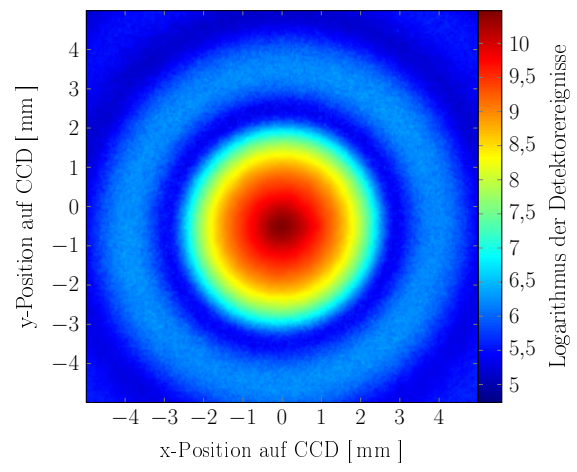

(b) Beugungsbild bei $260 \mathrm{eV}$ (Undulator UE52-SGM), bei 49,6 cm Abstand und $9 \mathrm{~s}$ Belichtungszeit.

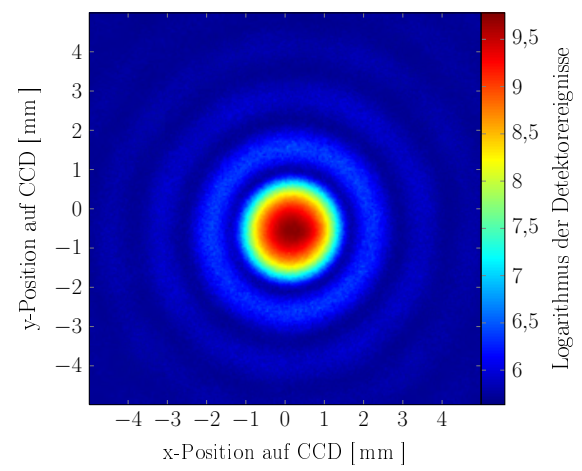

(d) Beugungsbild bei $500 \mathrm{eV}$ (Undulator UE52-SGM), bei $49,6 \mathrm{~cm}$ Abstand und 0,25 s Belichtungszeit.

\section{Abbildung 4.5:}

Zusammenstellung der Rohdaten von Beugungsbildern einer ultra-kleinen Lochblende (siehe Abbildung 4.6(e)) bei verschiedenen Photonenenergien.

trische Kreise, wie man sie von den Airyscheibchen einer Lochblende erwartet. Mit Hilfe einer Matlab-Routine (Anhang D auf Seite 145) wird das Detektorbild eingelesen und dessen Mittelpunkt festgelegt. Um die stark verschiedenen Intensitäten der verschiedenen Beugungsringe gemeinsam darzustellen, werden die Messwerte logarithmiert. In Abbildung 4.5 und 4.6 sind die Daten verschiedener Lochblenden bei mehreren Photonenenergien dargestellt. Ausgehend hiervon werden die Lage der Minima und Maxima auf Strahlen vom Mittelpunkt aus bestimmt und die Ergebnisse gemittelt. Diese Punkte werden mittels ,spline“-Interpolation verbunden. Ein Vergleich mit der theoretischen Lochblendenfunktion in Abbildung 4.7(b) zeigt die qualitativ gute Wiedergabe der so bestimmten Lage der Minima und Maxima sowie des Funktionsverlaufs. 


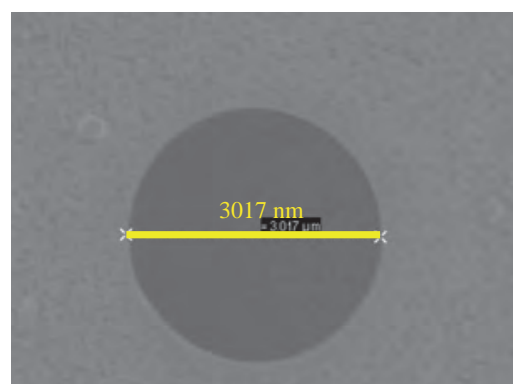

(a) REM-Bild der $3 \mu \mathrm{m}$ Lochblende (Zeiss LEO Supra 35, 5kV Spannung, $27890 \times$ Vergrößerung)

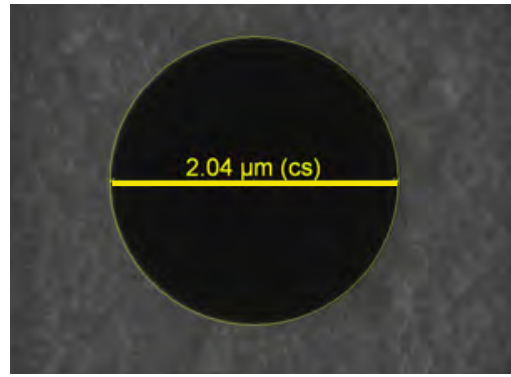

(c) REM-Bild der $2 \mu \mathrm{m}$ Lochblende (FEI Nova Nanolab, 5kV Spannung, $80000 \times$ Vergrößerung)

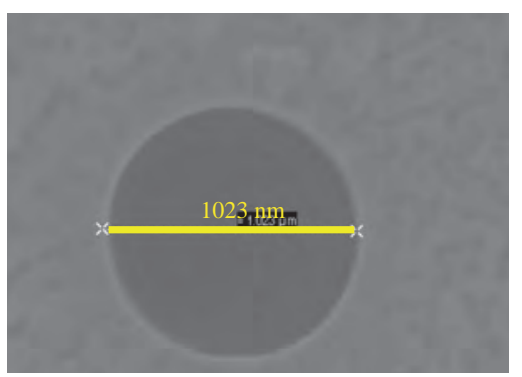

(e) REM-Bild der $1 \mu \mathrm{m}$ Lochblende (Zeiss LEO Supra 35, 5kV Spannung, $82220 \times$ Vergrößerung)

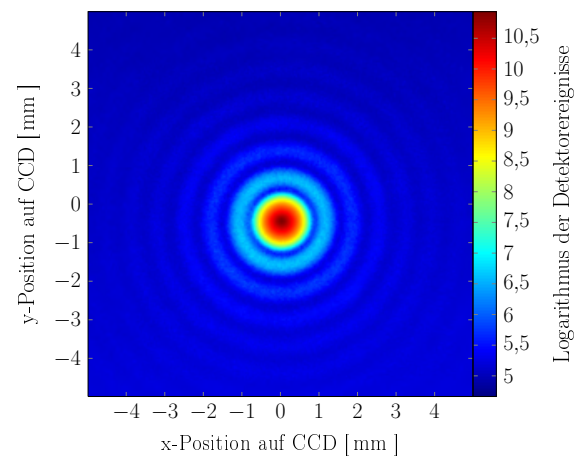

(b) Beugungsbild von (a) bei $260 \mathrm{eV}$ (Undulator UE52-SGM), bei $49,6 \mathrm{~cm}$ Abstand und $0,2 \mathrm{~s}$ Belichtungszeit.

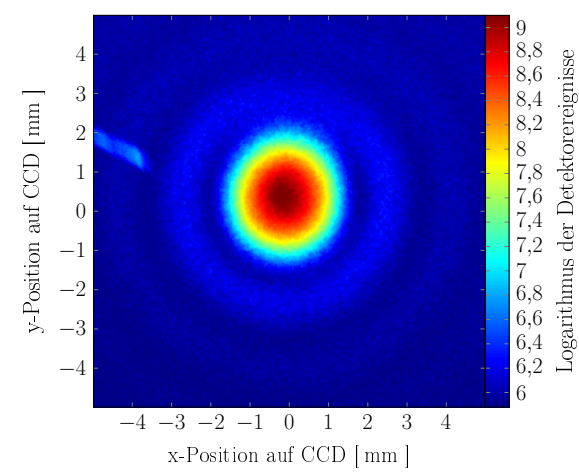

(d) Beugungsbild von (c) bei $200 \mathrm{eV}$ (Undulator U49/2-PGM1), bei 18,8 cm Abstand.

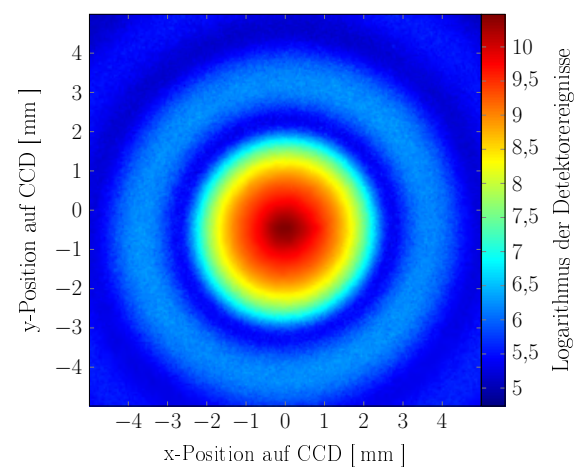

(f) Beugungsbild von (a) bei $260 \mathrm{eV}$ (Undulator UE52-SGM), bei 49,6 cm Abstand und $9 \mathrm{~s}$ Belichtungszeit .

\section{Abbildung 4.6:}

Zusammenstellung der Rohdaten von Beugungsbildern ultra-kleiner Lochblenden bei verschiedenen Durchmessern und vergleichbarer Photonenenergie. 


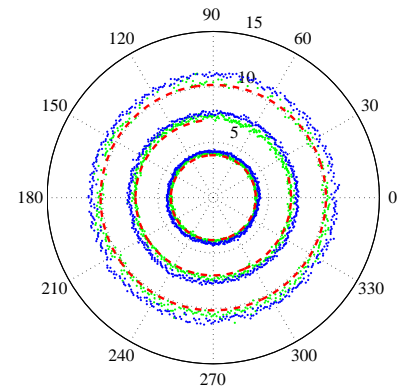

(a) Auswertung der q-Raumlage der Minima zwischen zwei Beugungsringen einer ultra-kleinen Lochblende (exemplarisch für Abbildung 4.6(e), $1 \mu \mathrm{m}$ Durchmesser bei $260 \mathrm{eV}$ - Undulator UE52-SGM). Grün gekennzeichnet sind die Winkel im q-Raum (in $1 / \mu \mathrm{m}$ ) der $1470 \mathrm{~nm}$ starken Folie, blau die der $1860 \mathrm{~nm}$ starken Folie.

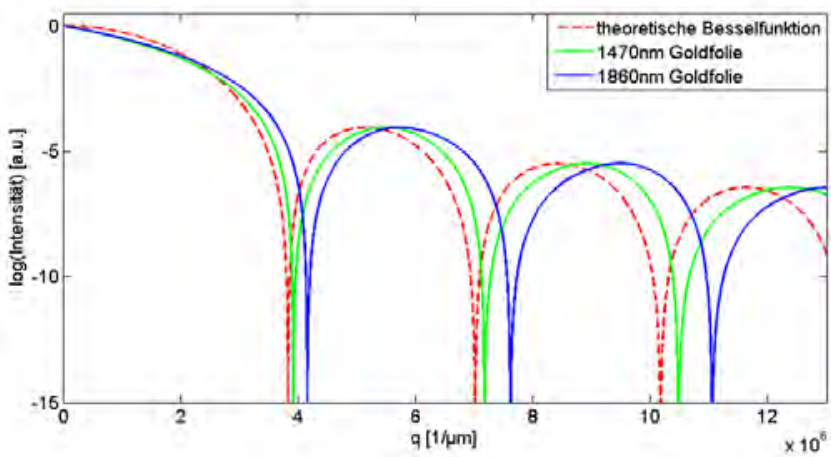

(b) Auftragung des durch Bestimmung der Mittelwerte der Minima und Maxima interpolierten Schnitts durch das Intensitätsprofil des Beugungsbildes.

\section{Abbildung 4.7:}

Die Auswertung der Beugungsringe hinter einer ultra-kleinen Lochblende erfolgt über die Lagebestimmung der Minima und Maxima zwischen den Beugungsringen. Sie können mit den Erwartungswerten der erwarteten Intensitätsverteilung aus den Herstellungsparameter verglichen werden. Diese sind ebenfalls aufgetragen.

Da aus experimentellen Gründen für die ultra-kleinen Lochblenden $500 \mathrm{~nm}$ Durchmesser keine aussagekräftigen Daten bei $92 \mathrm{eV}$ erhoben wurden, werden sie in die weitere Betrachtung nicht mit einbezogen. Für die Datenanalyse kommen die Programme R [R Development Core Team, 2011] und Statistica ${ }^{\circledR}$ [StatSoft, Inc., 2011] zum Einsatz. Abbildungen 4.8 und 4.9 zeigen die q-Raum-Lage des Radius des 1. Beugungsrings. Durch die Berechnung der Lage der Minima in q-Koordinaten werden die Daten um den Einfluss der Wellenlänge bereinigt. Diese Darstellung erlaubt somit einen direkten Vergleich der abweichenden Eigenschaften einer ultra-kleinen Lochblende bei verschiedenen Wellenlängen, denn das Beugungsfeld der Airyscheibchen einer Lochblende ist in q-Koordinaten für alle Wellenlängen gleich.

Systematische Unterschiede bei verschiedenen Wellenlängen in den Beugungsbildern deuten auf einen Zusammenhang mit der relevanten Tiefe und den Materialeigenschaften hin. Der kritische Durchmesser bei $92 \mathrm{eV}$, der die notwendige Beachtung wellenleitender Eigenschaften anzeigt [Fuhse und Salditt, 2006b], ist bei der $1 \mu \mathrm{m}$-Lochblende in der gleichen Größenordnung wie der tatsächliche Durchmesser. Man beobachtet dort eine deutliche Veränderung der Beugungsbilder im Vergleich mit den anderen Energien. 


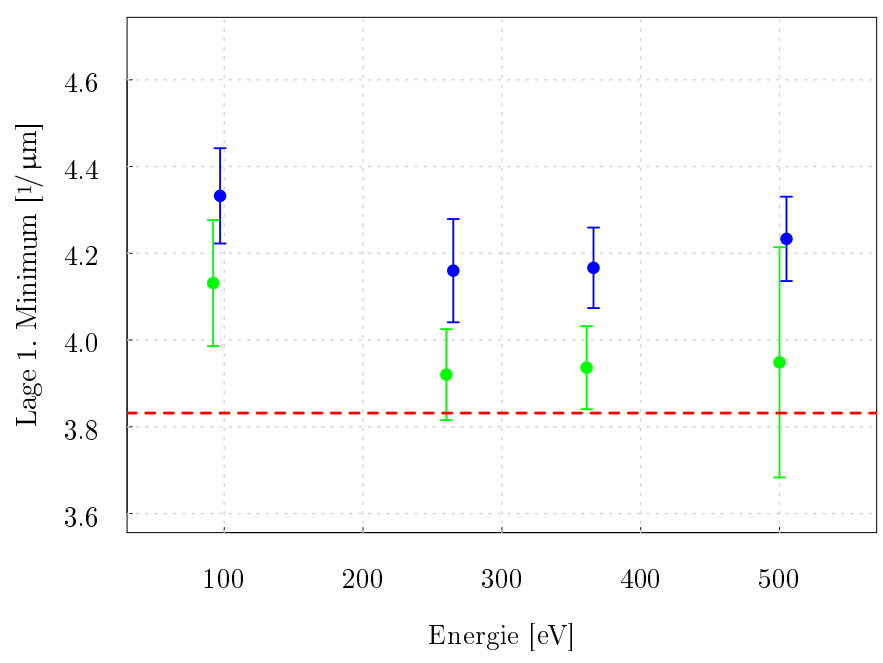

Abbildung 4.8:

q-Raumlage des ersten Beugungsminimums der $1 \mu \mathrm{m}$ großen ultra-kleinen Lochblende. Die blauen Datenpunkte sind die in der $1,86 \mu \mathrm{m}$ starken Folie, die grünen die in der 1,47 $\mu \mathrm{m}$ starken Folie. Die rote Linie zeigt die q-Raum-Lage des Designparameters an. Es zeigt sich eine signifikante Abweichung in der Lage des 1. Beugungsminimums bei $92 \mathrm{eV}$ Photonenenergie. Dies deutet auf einen wellenlängigen Effekt hin, der das Beugungsbild verändert, und wird als einsetzende wellenleitende Eigenschaft interpretiert $d / l \approx \theta_{c}$.

Eine alternative Betrachtungsweise besteht darin, die Beugungsbilder der ultrakleinen Lochblenden als Beugungsbilder von einer klassischen Lochblende, also einer Absorptionsmaske, zu interpretieren, da diese in Art und Ausprägung eng verwandt sind. $\mathrm{Zu}$ einem beobachteten Beugungsbild wird der Durchmesser der mathematischen Lochblende bestimmt, die dieses Beugungsbild verursacht hätte. Da stets die gleichen ultra-kleinen Lochblenden mit verschiedenen Photonenenergien beleuchtet wurden, sind verschiedene resultierende Radien auf eine von der Wellenlänge abhängige Eigenschaft der Löcher zurückzuführen.

Die stärkere Beugung lässt sich über die mathematische Beschreibung einer Lochblende in einem effektiven Lochblendendurchmesser angeben, wie in Abbildung 4.10 dargestellt. Es zeigt sich, dass der resultierende Durchmesser bei den höheren Photonenenergien beinahe konstant ist. Bei $92 \mathrm{eV}$ erscheinen jedoch die Lochdurchmesser jeweils um etwa $40 \mathrm{~nm}$ kleiner.

Bei geeigneter Photonenenergie zeigen beide etwa $1 \mu \mathrm{m}$ große ultra-kleinen Lochblenden einsetzende wellenleitende Eigenschaften und können nicht mehr als reine Lochblenden gesehen werden. Wie erwartet zeigen die $3 \mu \mathrm{m}$-Lochblenden diese Eigenschaft nicht. 


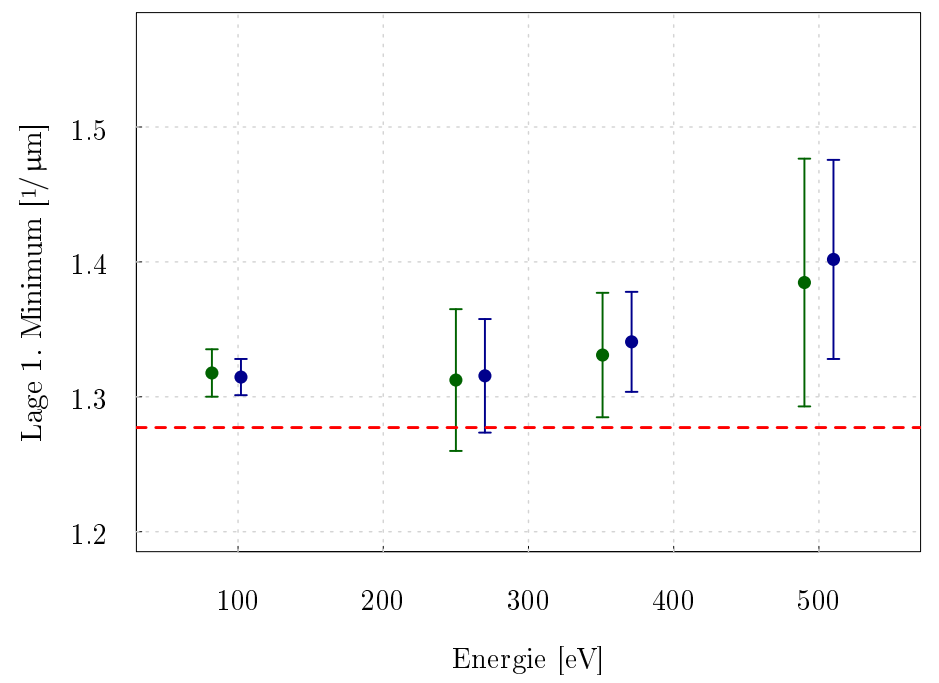

Abbildung 4.9:

q-Raumlage des ersten Beugungsminimums der $3 \mu \mathrm{m}$ großen Lochblende. Die blauen Datenpunkte sind die in der $1,86 \mu \mathrm{m}$ starken Folie, die grünen die in der 1,47 $\mathrm{m}$ starken Folie. Die rote Linie zeigt die q-RaumLage des Designparameters an. Die Abweichung der Lagen der $500 \mathrm{eV}$ Beugungsminima erklärt sich aus der Transmission des Synchrotronstrahls durch die Folie. Da sich diese größeren Lochblenden wie reine Absorptionsmasken verhalten $\left(d / l \gg \theta_{c}\right)$, treten keine Abweichungen auf.

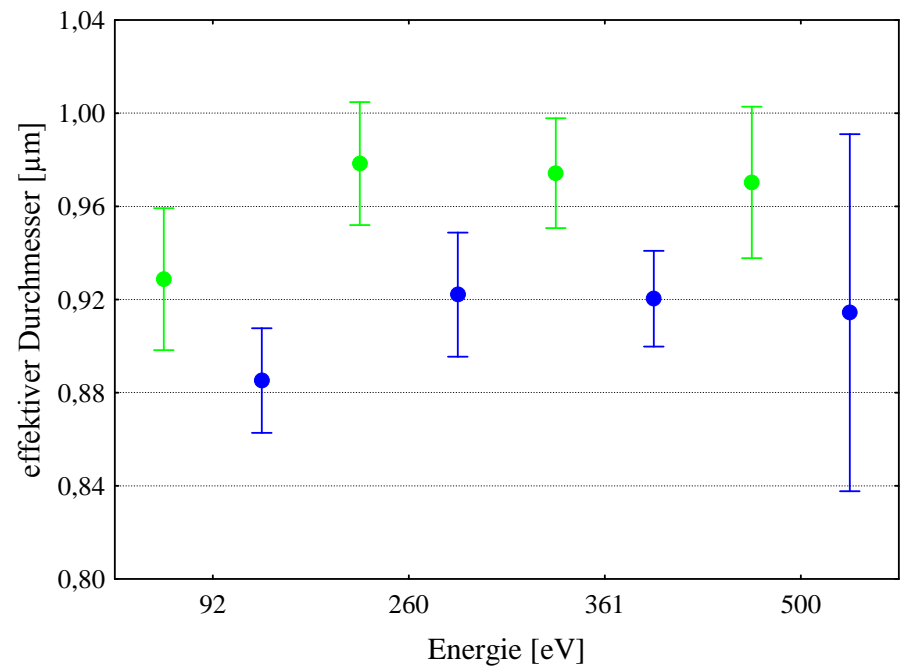

Abbildung 4.10:

Effektiver Durchmesser der $1 \mu \mathrm{m}$ ultra-kleinen Lochblende bei vier verschiedenen Photonenenergien. Die blauen Datenpunkte sind die der Löcher in der $1,86 \mu \mathrm{m}$ starken Folie, die grünen gehören zu der 1,47 $\mu \mathrm{m}$ starken Folie. Die aus den Ablenkwinkeln bestimmten Durchmesser stimmen bei großen Photonenenergien gut mit rasterelektronenmikroskopisch bestimmten Durchmessern überein. Bei $92 \mathrm{eV}$ zeigt sich bei derselben ultra-kleinen Lochblende eine Abweichung von der erwarteten Beugungsfeldintensitätsverteilung einer Lochblende. Diese Darstellung entspricht der in Abbildung 4.8. 


\subsection{Ptychographic Coherent Diffractive Imaging}

Die Ptychographie ist eine neue Methode zur vollständigen Charakterisierung komplexer Wellenfelder, die dabei eine sehr hohe räumliche Auflösung erzielt [Rodenburg und Faulkner, 2004; Rodenburg u.a., 2007; Thibault u. a., 2008; Maiden und Rodenburg, 2009; Giewekemeyer u.a., 2010b; Dierolf u.a., 2010]. Zur Erzeugung eines ptychographischen Datensatzes werden die Fernfelder einer beugenden Probe ohne jegliche optischen Elemente hinter der Probe unter verschiedenen, jeweils mit Überlapp verschobener Beleuchtung aufgenommen. Die Namesgebung der Methode (griechisch: $\pi \tau \nu \xi$ - falten) stammt aus der diffraktiven Elektronenmikroskopie von Kristallen, bei der das beobachtete Fernfeld eines ausgedehnten Kristalls mit dem Fernfeld der Beleuchtung gefaltet wird. Aus der Überlagerung der verschiedenen „Bragg-Peaks“ kann die Phaseninformation an diesen aus den Beugungsbildern zurück gewonnen werden [Hoppe, 1969]. Eine iterative Implementation durch Rodenburg und Faulkner [2004] (PIE - Ptychographic Iterative Engine) ermöglicht den Einsatz in der diffraktiven Bildgebung. Durch seine hohe Überbestimmtheit bei stark überlappend beleuchteten Bereichen der Probe weist dieser sehr gute Rekonstruktionseigenschaften auf. Da die Beleuchtung der Probe immer wieder verschoben wird, ist die Größe der untersuchten Probe nicht durch den Algorithmus limitiert.

Unter genauer Kenntnis der beleuchtenden Funktion $P(\overrightarrow{\mathbf{r}})$, wobei $\overrightarrow{\mathbf{r}}$ den Abstand von der optischen Achse und $\overrightarrow{\mathbf{R}}$ die Verschiebung der Beleuchtungsfunktion beschreibt, kann das komplexe Wellenfeld $\Psi(\overrightarrow{\mathbf{r}}, \overrightarrow{\mathbf{R}})$ hinter einem streuenden Objekt mit Transmissionsfunktion $O(\overrightarrow{\mathbf{r}})$ prinzipiell durch

$$
\Psi(\overrightarrow{\mathbf{r}}, \overrightarrow{\mathbf{R}})=O(\overrightarrow{\mathbf{r}}) P(\overrightarrow{\mathbf{r}}-\overrightarrow{\mathbf{R}})
$$

berechnet werden. Thibault u. a. [2008] erweitern den Algoritmus neben dem Auffinden der komplexen Objektfunktion auf das gleichzeitige Auffinden der komplexen Beleuchtungsfunktion, die in dieser Arbeit zur Betrachtung der optischen Eigenschaften der ultra-kleinen Lochblenden näher untersucht wird. Zudem soll ebenfalls ein kurzer Überblick der Ergebnisse der mikroskopischen Auswertung der untersuchten Probe gegeben werden. Die Methode wurde bereits zuvor erfolgreich auf verschiedene andere Röntgenoptiken angewandt [Thibault u.a., 2008; Schropp u.a., 2010; Kewish u.a., 2010; Giewekemeyer u. a., 2010a].

Eine gute Schätzung der Beleuchtungsfunktion für den Algorithmus ist dabei für eine schnelle und hochwertige Rekonstruktion von schwach streuenden biologischen Proben sehr hilfreich, da sie eine vorherige Bestimmung mit einer stark streuenden Struktur, wie die eines Siemenssterns [Dierolf u.a., 2010], unnötig macht. Die wohldefinierten ultra-kleinen Lochblenden erlauben eine genaue Schätzung der Beleuchtungsfunktion 
und eignen sich dadurch besonders für den Einsatz in der ptychographischen Mikroskopie mit weicher Röntgenstrahlung.

\subsubsection{Algorithmus}

Für die Rekonstruktion wird der Algorithmus, wie er von Thibault u.a. [2008] vorgestellt wurde, angewandt. Die wesentliche Stärke dieses Algorithmus liegt darin, dass er neben der komplexen Objektfunktion $O(\overrightarrow{\mathbf{r}})$ auch die komplexe Beleuchtungsfunktion $P(\overrightarrow{\mathbf{r}})$ rekonstruiert (Gleichung 4.3). Dabei wird das resultierende Wellenfeld an jedem Aufnahmepunkt durch die Objektfunktion und die verschobene Beleuchtungsfunktion modelliert. Da das Wellenfeld nur im Fernfeld detektiert werden kann, wird unter Verlust der Phaseninformation an einer Position $j$ die Intensität der zweidimensionalen Fouriertransformation $\mathcal{F}\{\Psi(\overrightarrow{\mathbf{r}})\}$ gemessen, wobei der Streuvektor $\overrightarrow{\mathbf{q}}$ die Koordinate im reziproken Raum bezeichnet:

$$
I_{j}(\overrightarrow{\mathbf{q}})=\left|\mathcal{F}\left\{\Psi_{j}(\overrightarrow{\mathbf{r}})\right\}\right|^{2} .
$$

Der Algorithmus findet dann mit Hilfe eines geschätzten Startwerts iterativ eine Lösung zu Gleichung 4.3 an jeder Position $j$. Während der Iterationen wird immer wieder die Konsistenz zwischen Messung und Modell erzwungen, indem die Amplitude des gemessenen Wellenfeldes durch die Wurzel der gemessenen Intensitätsverteilungen ersetzt wird. Dabei wird die Phaseninformation iterativ zurückgewonnen. Für eine genauere Rekonstruktion müssen außerdem Störfaktoren wie Datenrauschen in Betracht gezogen werden [Giewekemeyer, 2011].

Das so rekonstruierte komplexe Wellenfeld mit $k$ als der Wellenzahl und $n$ als dem komplexen Brechungsindex gehorcht der Wellengleichung

$$
\left(\nabla^{2}+k^{2} n^{2}\right) \Psi_{\omega}(\overrightarrow{\mathbf{r}})=0,
$$

wobei $\Psi_{\omega}(\overrightarrow{\mathbf{r}})$ dabei ein stationäres monochromatisches Feld bezeichnet. In der paraxialen Näherung kann sie nach dem Superpositionsprinzip für beide Raumrichtungen näherungsweise gelöst werden [Paganin, 2006; Giewekemeyer, 2011]:

$$
\Psi_{\omega}\left(r_{\perp}, z\right) \cong e^{i k z} \mathcal{F}_{\perp}^{-1}\left[\exp \left(\frac{-i z k_{\perp}^{2}}{2 k}\right) \mathcal{F}_{\perp}\left(\Psi_{\omega}\left(r_{\perp}, 0\right)\right)\right] .
$$

$k_{\perp}$ bezeichnet hier die Fourierraumkoordinate. Mit Hilfe dieser Gleichung lässt sich das komplexe Wellenfeld in verschiedene Ebenen propagieren, so dass Rekonstruktion und später die Simulation (Kapitel 4.4) einfach miteinander verglichen werden können. 


\subsubsection{Versuchsaufbau}

Die ptychographischen Datensätze wurden bei zwei Messzeiten am Speicherring BESSY II des Helmholzzentrums in Berlin an den Undulatorstrahlungsquellen U49/2-PGM1 (September 2009) und UE52-SGM (Juni 2010) durchgeführt. Dazu wurde eine einzelne der ultra-kleinen Lochblenden belichtet. In einer geringen Entfernung von 0,7-1,0 mm. Dahinter befand sich die Probe. Deren Beugungsfeld wurde im Fernfeld von einer rückseitig gedünnten CCD-Kamera (Andor Technology DX436, 2048 ${ }^{2}$ Pixel, 13, $5^{2} \mu \mathrm{m}^{2} \mathrm{Pi}$ xelgröße) in einem Abstand von 18 beziehungsweise $28 \mathrm{~cm}$ detektiert.

In diesen Experimenten wurde ein 2,04 $\mu \mathrm{m}$ im Durchmesser messende ultra-kleine Lochblende bei $530,8 \mathrm{eV}$ charakterisiert, indem eine ptychographische Mikroskopaufnahme eines Modellsystems aus Polymethylmethacrylat (PMMA)- und Siliziumdioxid $\left(\mathrm{SiO}_{2}\right)$-Kugeln von $2 \mu \mathrm{m}$ Durchmesser angefertigt wurde. Es wurden $18 \times 15$ Beugungsbilder mit jeweils $800 \mathrm{~nm}$ Abstand aufgenommen. Ein Beispiel dieser Aufnahmen findet sich in Abbildung 4.11(a).

\subsubsection{Ergebnis Rekonstruktion}

Die ptychographisch rekonstruierte komplexe Beleuchtungsfunktion wurde mit Hilfe der

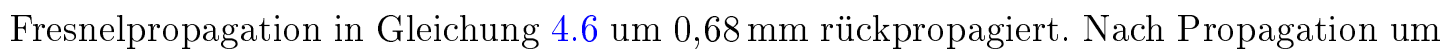
diese Entfernung erreicht man das komplexe Wellenfeld am Ausgang der ultra-kleinen Lochblende, die in Abbildung 4.11(b) dargestellt ist. Es zeigt sich ein kreisförmiges Wellenfeld mit konstanter Phase. Ein Vergleich mit einer in Abbildung 4.11(c) dargestellten elektronenmikroskopischen Aufnahme des Ausgangs der Lochblende zeigt, dass die Durchmesser der beiden gut übereinstimmen. Die aus den Beugungsbildern einer schwach streuenden Probe rekonstruierte Beleuchtungsfunktion ist also sehr plausibel und stimmt gut mit den Erwartungen überein.

\subsubsection{Chemischer Kontrast in der Ptychographie mit weicher Röntgenstrahlung}

Die eigentliche Zielsetzung des ptychographischen Experiments war nicht das Auffinden der komplexen Beleuchtungsfunktion, sondern der komplexen Objektfunktion. Deshalb wird an dieser Stelle ein Überblick über die in dieser Kooperation und durch Beckers u. a. [2011] veröffentlichten Ergebnisse der ptychographischen Mikroskopaufnahmen gegeben.

Eine herausragende Eigenschaft von weicher Röntgenstrahlung ist die Möglichkeit, über die Transmissions- und Phaseninformation der bei verschiedenen Wellenlängen Probeneigenschaften, wie deren chemische Zusammensetzung zu untersuchen [Thieme u. a., 1998]. Auch in der Ptychographie kann diese Methode angewandt werden und wird dort als Resonanz-Ptychographie bezeichnet. In dem durchgeführten Experiment 


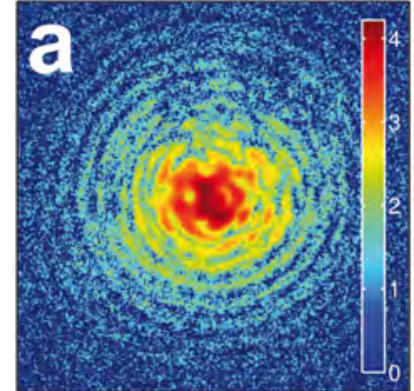

(a) Logarithmische Auftragung der zentralen $512 \times 512$ Pixel eines der 270 Beugungsbilder von der mit der Lochblende bei $530,8 \mathrm{eV}$ beleuchteten Probe.

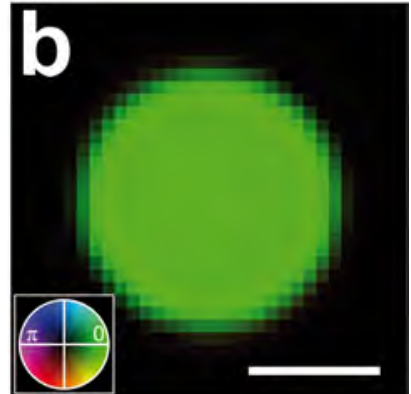

(b) Um 0,68 mm rückpropagiertes Wellenfeld der rekonstruierten Beleuchtungsfunktion. Die Darstellung ist im HSV-Farbraum kodiert. Die Amplitude ist durch Farbintensität und die Phase durch die Farbe dargestellt.

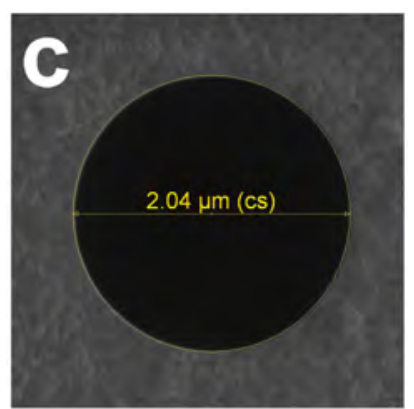

(c) Elektronenmikroskopische Aufnahme des Ausgangs der ultra-kleinen Lochblende.

Abbildung 4.11:

Rekonstruktion des komplexen Wellenfeldes am Ausgang der ultra-kleinen Lochblende. Es zeigt sich eine sehr gute Übereinstimmung der Größe der praktisch ebenen Wellenfront der rekonstruierten und rückpropagierten Beleuchtungsfunktion mit der elektronenmikroskopischen Voruntersuchung [Beckers u. a., 2011].

wurden die Resonanzen der NEXAFS-K-Kante von Sauerstoff genutzt, um in einem Modellsystem zwei verschiedene, sauerstoffhaltige Proben zu unterscheiden (zur Versuchsdurchführung siehe 4.3.2). Dabei wurden Datensätze bei fünf Photonenenergien zwischen $530,0 \mathrm{eV}$ und 533,4 eV aufgenommen. Die rekonstruierten Objektfunktionen sind in Abbildung 4.12 aufgetragen.

Man beobachtet unterschiedliches Verhalten der PMMA- und $\mathrm{SiO}_{2}-\mathrm{Kügelchen} \mathrm{in} \mathrm{Ab-}$ sorption und Phasenschub. Unphysikalische Sprünge der Phase bei Phasendifferenzen größer $\pi$ werden durch geeignete Entfaltungsalgorithmen verhindert. So ist eine eindeutige Zuordnung der Kügelchen trotz der gleichen Struktur und Größe möglich ist. Die Aufösung der Rekonstruktionen wird nach Betrachtung einer Gausschen Punktspreizfunktion mit einer Halbwersbreite von 0,5 und 1,5 Pixeln in dem Bereich einer Pixelgröße angegeben und liegt bei $96 \mathrm{~nm}$.

Als eine mögliche Anwendung dieser Methodik wurde eine organische Probe, nämlich das Bakterium Deinococcus radiodurans mikroskopiert. Diese Bakterien haben die bemerkenswerte Eigenschaft ionisierender Strahlung in hohen Dosen widerstehen zu können, da sie in der Lage sind, Schäden an der Desoxyribonukleinsäure (DNS) herausragend gut zu reparieren. Diese Resistenz scheint mit der Anreicherung schützender Mangankomplexe um die DNS in Zusammenhang zu stehen [Levin-Zaidman u. a., 2003; Daly, 2009]. Ein einzelnes Bakterium wurde hinter einer $1 \mu \mathrm{m}$ Lochblende platziert und 


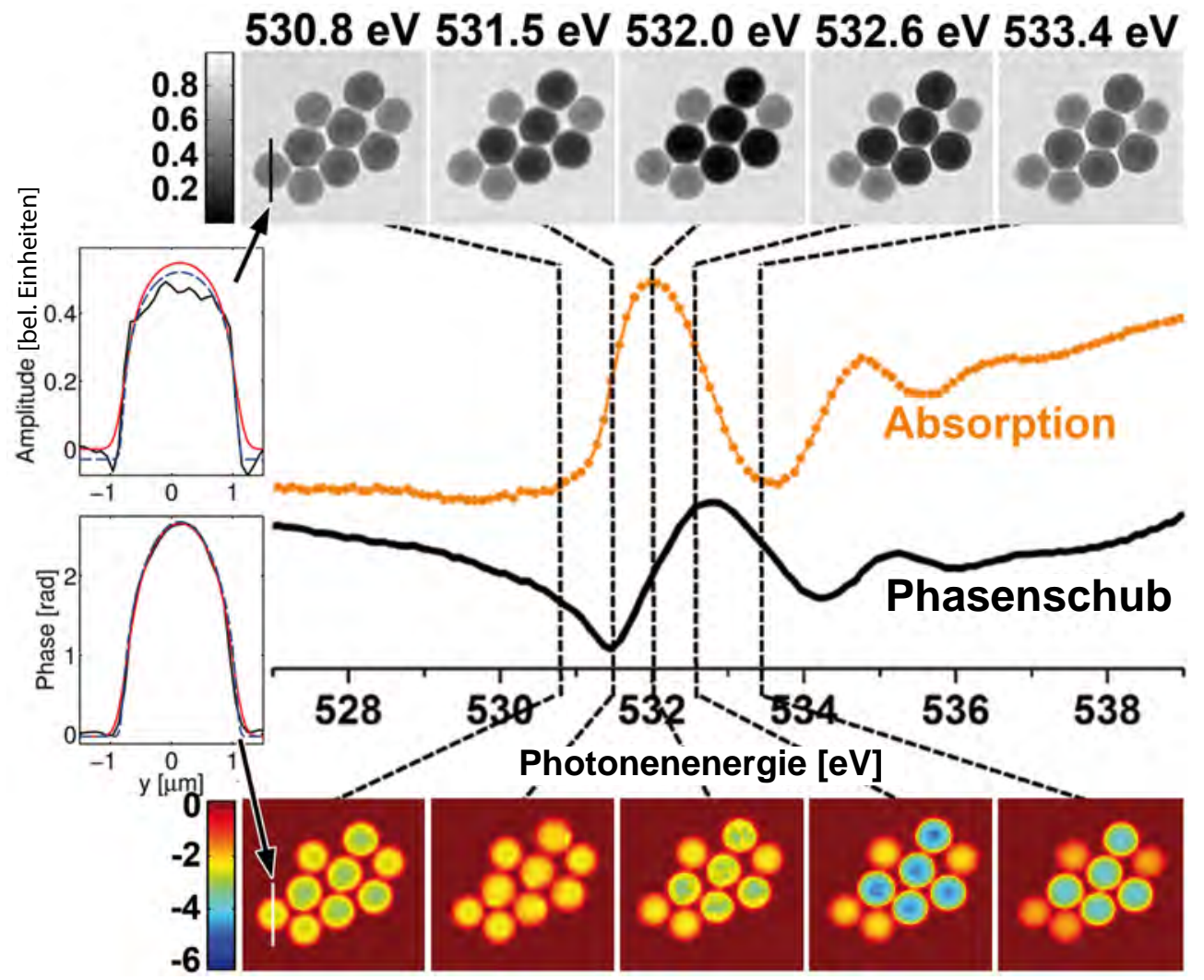

Abbildung 4.12:

Rekonstruktion der Objektfunktionen des $\mathrm{SiO}_{2-} / \mathrm{PMMA-Modellsystems} \mathrm{in} \mathrm{Amplitude} \mathrm{und} \mathrm{(entfalteter)} \mathrm{Pha-}$ se bei fünf verschiedenen Photonenenergien um das Absorptionsmaximum von PMMA. Die Kugeln lassen sich einfach durch den Phasenschub von PMMA in diesem Wellenlängenbereich unterscheiden. Zur Abschätzung der räumlichen Auflösung ist der Schnitt durch eine Kugel (schwarz) und der Schnitt mit einer Punktspreizfunktion mit Halbwertsbreiten von 0,5 (rot) und 1,5(blau) Pixeln aufgetragen gefaltet. [Beckers u. a., 2011]

ein ptychographischer Datensatz von $22 \times 24$ Bildern bei $600 \mathrm{~nm}$ Schrittweite und $517 \mathrm{eV}$ Photonenenergie aufgenommen. Die Rekonstruktion der inneren $15 \times 19$ Bilder ist in Abbildung 4.13 gezeigt.

Die rekonstruierte Objektfunktion ist konsistent mit der lichtmikroskopischen Aufnahme. Unter Annahme eines Modellproteins $\left(\mathrm{H}_{50} \mathrm{C}_{30} \mathrm{~N}_{9} \mathrm{O}_{10} \mathrm{~S}_{1}\right)$, das ein Drittel des Volumens der Zelle ausmache, lässt sich die Dicke der Zelle anhand des Phasenschubs mit 1-2 $\mu \mathrm{m}$ abschätzen. Diese ptychographische Mikroskopaufnahme einer biologischen Probe mit Strahlung innerhalb des Wasserfensters ist die erste ihrer Art. 


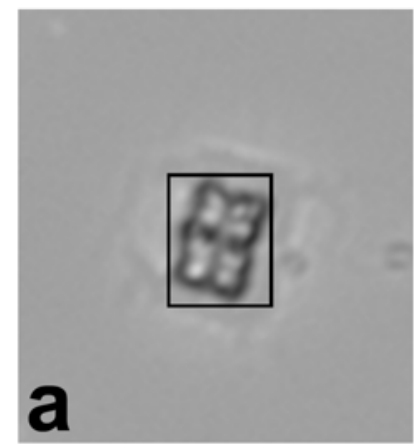

(a) Lichtmikroskopische Aufnahme des untersuchten Bakteriums. Der ptychographisch untersuchte Bereich ist markiert.

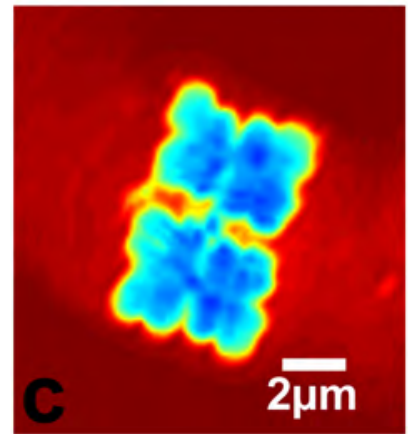

(c) Rekonstruktion der Objektfunktion (entfaltete Phase).

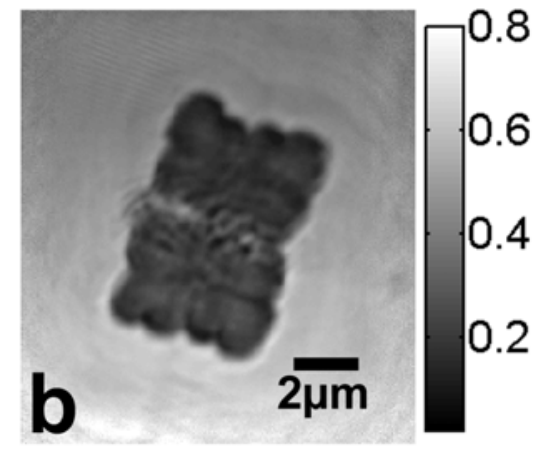

(b) Rekonstruktion der Objektfunktion (Amplitude).

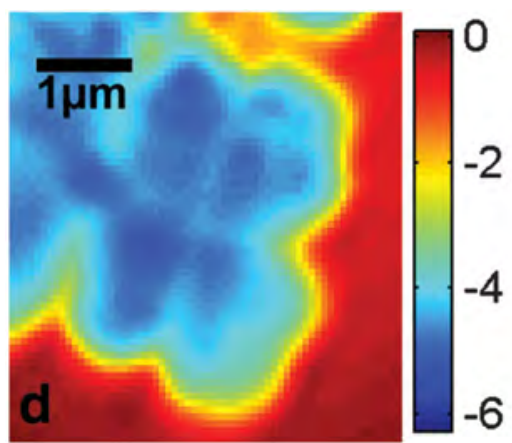

(d) Rekonstruktion der Objektfunktion (entfaltete Phase) im Detail.

Abbildung 4.13:

Rekonstruktion der Objektfunktionen eines Deinococcus radiodurans-Bakteriums [Beckers u. a., 2011]. 


\subsection{Simulation}

Um die optischen Eigenschaften der ultra-kleinen Lochblenden näher zu untersuchen und zu überprüfen, inwiefern und bei welchen Wellenlängen wellenleitende Eigenschaften eine Rolle spielen, werden eine Reihe von Simulationen durchgeführt und insbesondere die Rekonstruktion aus Kapitel 4.3.3 mit den simulierten Ergebnissen verglichen.

\subsubsection{Das finite-Differenzen Programm Planar Wave Guide}

Zur Simulation des Wellenfelds wird das Programm Planar Wave Guide von Christian Fuhse und Markus Osterhoff eingesetzt [Fuhse und Salditt, 2006b; Fuhse, 2006; Osterhoff und Salditt, 2009].

Um die Wellengleichung (Gleichung 4.5) numerisch für einen Röntgenwellenleiter zu berechnen, bedarf es einer geeigneten Approxmation, da aufgrund der kurzen Wellenlänge ein nicht realisierbar großes Stützgitter angelegt werden müsste. Bei einer Welle, die sich nahe der optischen Achse in z-Richtung ausbreitet, kann das sich ausbreitende Feld durch ein Produkt von $e^{-i k x}$ und einer Funktion $u(x, y, z)$ ausgedrückt werden.

$$
\Psi(x, y, z)=u(x, y, z) e^{-i k z}
$$

Da diese Funktion $u$ deutlich langsamer oszilliert, lässt sich das Stützgitter gröber und damit numerisch beherrschbar gestalten. Unter Vernachlässigung der Ableitungen zweiter Ordnung, eingesetzt in die Wellengleichung (Gleichung 4.5), ergibt sich die parabolische Wellengleichung:

$$
-2 i k \frac{\partial u}{\partial x}+\left(\frac{\partial^{2}}{\partial y^{2}}\right) u+k^{2}\left(n^{2}-1\right) u=0
$$

Diese wurde in IDL ${ }^{\complement}$ [ITT Visual Information Solutions] für ein in einer Raumrichtung eingeschlossenen Wellenleiter implementiert und auf ihre Genauigkeit erfolgreich überprüft [Fuhse und Salditt, 2006a; Fuhse, 2006]. Eine Reprogrammierung in der Programmiersprache $C$ erfolgte durch Osterhoff und Salditt [2009].

\subsubsection{Simulation der ultra-kleinen Lochblenden}

Für verschiedene Wellenlängen werden Simulationen der Wellenfelder in den ultrakleinen Lochblenden durchgeführt. Dabei wird geprüft, ob einsetzende Wellenleitung für die in Kapitel 4.2.2 aufgefundenen Eigenschaften verantwortlich ist. Die Simulationen werden in erster Näherung für eine eindimensionale Geometrie durchgeführt. 


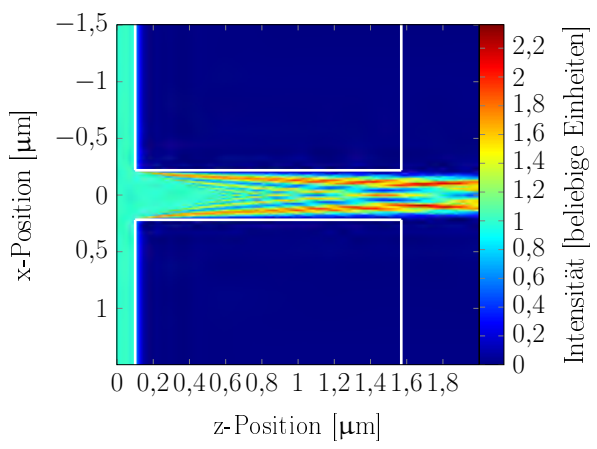

(a) ultra-kleine Lochblende, $437 \mathrm{~nm}$ Durchmesser, $1,47 \mu \mathrm{m}$ Tiefe, $92 \mathrm{eV}$ Photonenenergie.

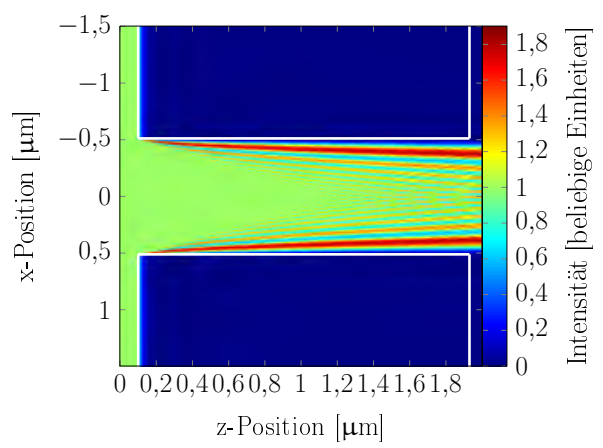

(c) ultra-kleine Lochblende, $905 \mathrm{~nm}$ Durchmesser, $1,86 \mu \mathrm{m}$ Tiefe, $92 \mathrm{eV}$ Photonenenergie.

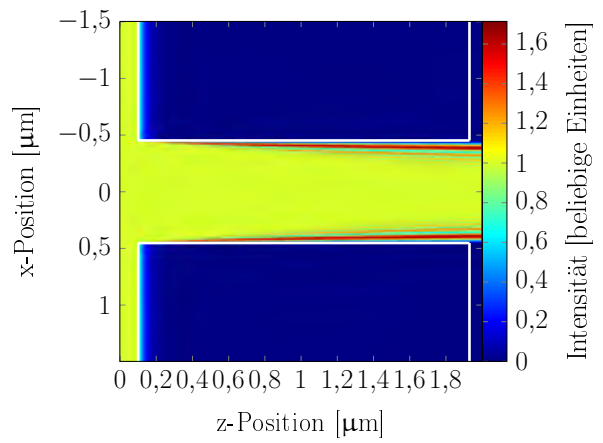

(e) ultra-kleinen Lochblende, $905 \mathrm{~nm}$ Durchmesser, $1,86 \mu \mathrm{m}$ Tiefe, $361 \mathrm{eV}$ Photonenenergie.

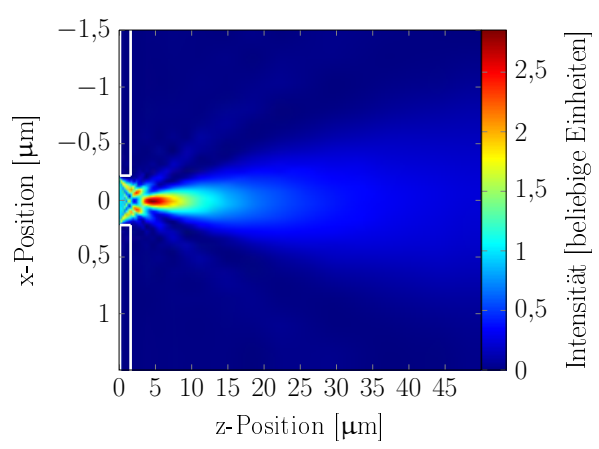

(b) „Pseudofokus“, $437 \mathrm{~nm}$ Durchmesser, 1,47 $\mu \mathrm{m}$ Tiefe, $92 \mathrm{eV}$ Photonenenergie.

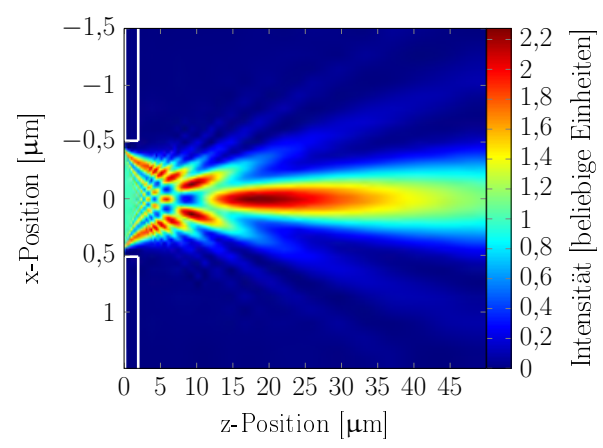

(d) „Pseudofokus“, $905 \mathrm{~nm}$ Durchmesser, $1,86 \mu \mathrm{m}$ Tiefe, $92 \mathrm{eV}$ Photonenenergie.

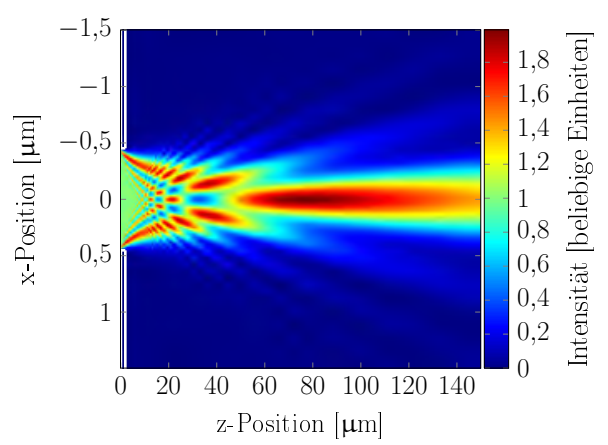

(f) „Pseudofokus“, $905 \mathrm{~nm}$ Durchmesser, $1,86 \mu \mathrm{m}$ Tiefe, $361 \mathrm{eV}$ Photonenenergie.

\section{Abbildung 4.14:}

Simulation mit dem von Osterhoff und Salditt [2009] in C verfasstem Programm Planar Wave Guide. Es werden verschiedene ultra-kleine Lochblenden unter Variation von Durchmesser, Tiefe und Photonenenergie untersucht. Wie aus den Messungen in Kapitel 4.2.2 vermutet, sind die wellenleitenden Eigenschaften für kleine, tiefe Löcher bei geringer Photonenenergie, das heißt einem großen kritischen Winkel, am stärksten. Hinter allen simulierten Lochblenden bildet sich ein sogenannter „Pseudofokus“ aus [Fuhse und Salditt, 2006b]. 


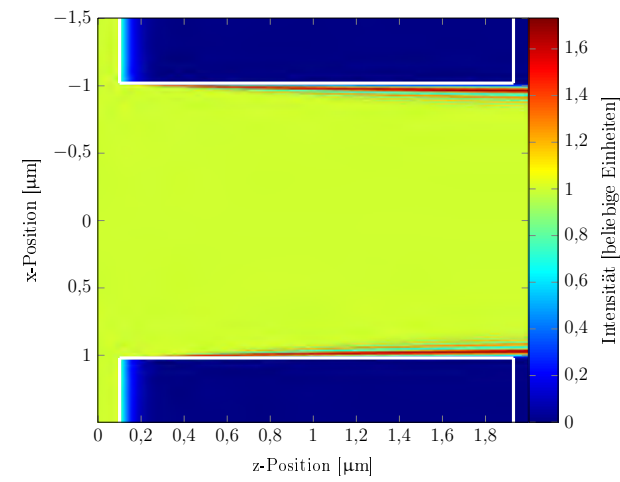

(a) ultra-kleine Lochblende, 2,04 $\mu \mathrm{m}$ Durchmesser, $1,86 \mu \mathrm{m}$ Tiefe, $530,8 \mathrm{eV}$ Photonenenergie.

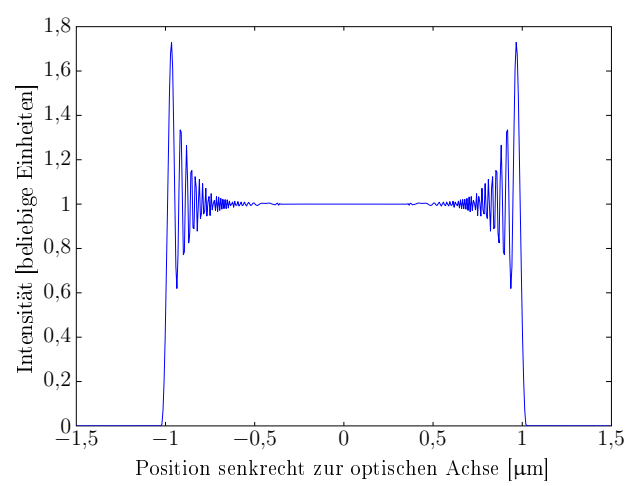

(b) zugehörige Intensitätsverteilung des Wellenfeldes am Ausgang der ultra-kleinen Lochblende.

Abbildung 4.15:

Simulation der ultra-kleinen Lochblenden aus dem Ptychographie-Experiment. Die wellenleitenden Eigenschaften sind nur gering. Dennoch zeigen sich starke Modulationen der Intensität des Wellenfeldes an der inneren Wand.

In Abbildung 4.14 sind die Simulationen für eine Auswahl verschiedener Durchmesser, Tiefen und Wellenlängen in der ultra-kleinen Lochblende selbst und dem Ausbreitungsbereich dahinter gezeigt, in dem eine sogenannte „Pseudo-Fokussierung“" stattfindet, die interessante Anwendungen in Bildgebung oder Lithografie haben könnte [Fuhse und Salditt, 2006b]. Das einfallende Röntgenlicht des Synchrotron wurde dabei als ebene Welle angenähert.

Für einen genaueren Vergleich der Simulationen mit den rekonstruierten Feldern wird auch der aus den ptychographischen Rekonstruktionen gewonnene Datensatz mit der Simulation verglichen. Die zugehörige Simulation einer 2,04 $\mu \mathrm{m}$ großen Lochblende bei $530,8 \mathrm{eV}$ ist in Abbildung 4.15(a) dargestellt.

Um diesen Vergleich durchzuführen, wird das simulierte Wellenfeld am Ausgang des Wellenleiters 4.15 (b) um $0,78 \mathrm{~mm}$ propagiert und dort mit einem Schnitt durch das Zentrum der praktisch rotationssymmetrischen, rekonstruierten Beleuchtungsfunktion verglichen. Diese beiden Kurven sind in Abbildung 4.16 dargestellt.

Es zeigt sich ein gute Übereinstimmung zwischen Simulation und Experiment. Die Abweichungen bei den höheren Beugungsordnungen können im Wesentlichen durch die eindimensionale Simulation der ultra-kleinen Lochblenden sowie mögliche, nicht exakt bekannte Ungenauigkeiten in der Herstellung und Modellierung der Lochblende erklärt werden. 


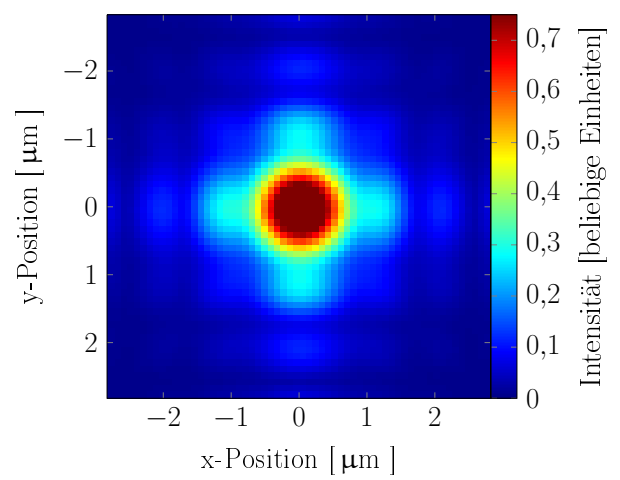

(a) Rekonstruktion der Beleuchtungsfunktion aus den Ptychographiescan. Das scheinbar unterliegende Netz hat ein $800 \mathrm{~nm}$ Raster, das der Verfahrweite während der Scan entspricht und vermutlich daher rührt.

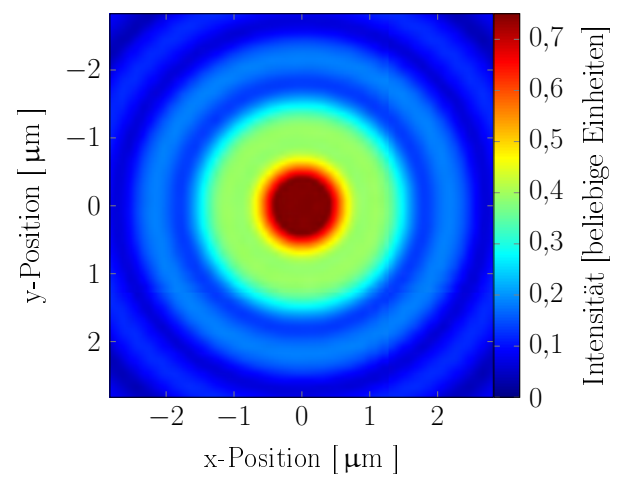

(b) Rotationssymmetrisch dargestelltes Ergebnis der Simulation mit Planar Wave Guide und nach der Propagation in Probenebene.

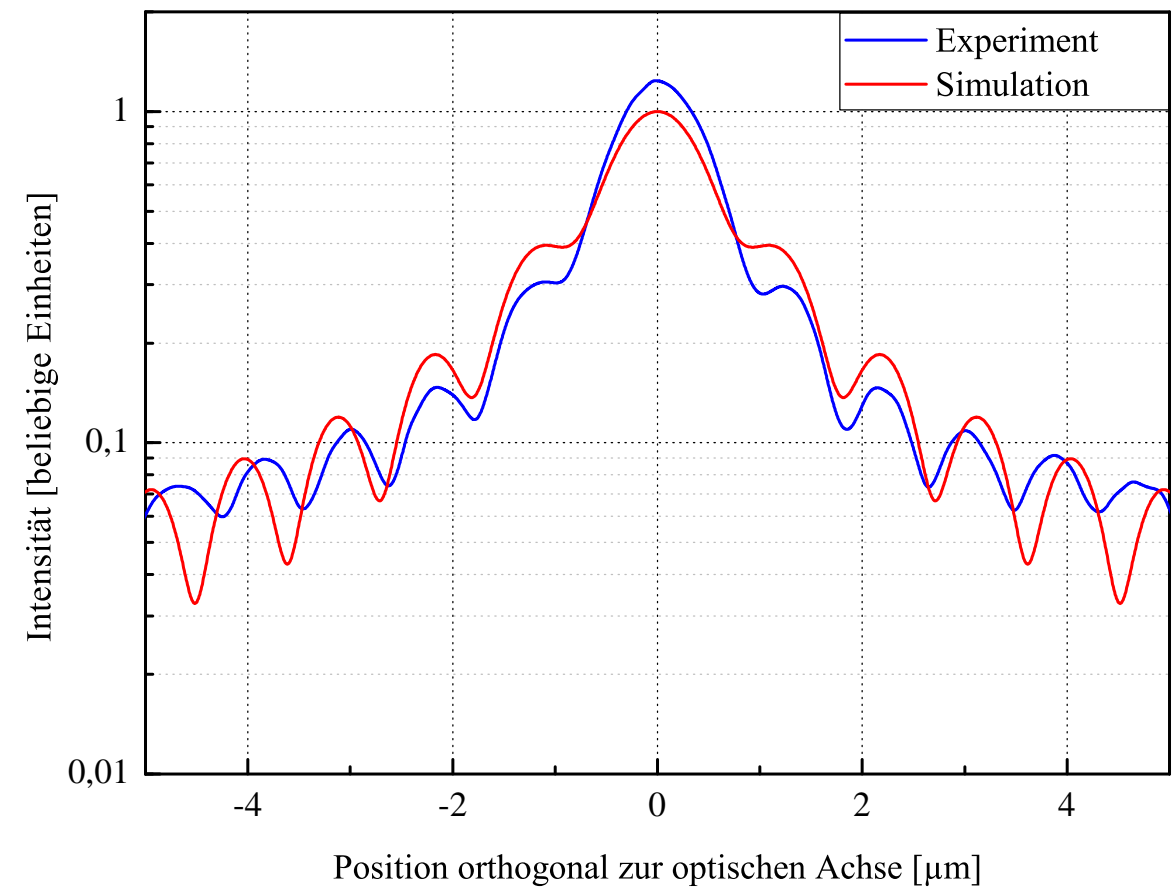

(c) Vergleich der propagierten Simulation mit einem horizontalen Schnitt durch die rekonstruierte Beleuchtungsfunktion.

\section{Abbildung 4.16:}

Vergleich der aus der ptychographischen Rekonstruktion und den Wellenleitersimulationen nach einer Propagation über $0,78 \mathrm{~mm}$ gewonnenen Probenfunktion. Zur besseren Vergleichbarkeit wurden die Amplituden der Kurven zueinander skaliert. Es zeigt sich eine sehr gute Übereinstimmung, die bei größeren Abständen von der optischen Achse leicht schlechter wird. Eine Abschätzung über den Fresnelparameter $F=d^{2} / z \lambda=2 \mu \mathrm{m}^{2} / 0,7 \mathrm{~mm} \cdot 2,4 \mathrm{~nm}=2,4 \approx 1$ zeigt, dass die Probenebene in $0,7 \mathrm{~mm}$ Entfernung im Bereich der Fresnel-Beugung befindet. 


\subsection{Vergleich der Ergebnisse}

Die durchgeführten Experimente und Simulationen zeigen, dass in engen und tiefen Lochblenden bei geringer Photonenenergie wellenleitende Eigenschaften beobachtet werden können. Durch ihre genau definierte Form und Größe eignen sie sich hervorragend für die ptychographische Röntgenmikroskopie von schwach streuenden Proben.

Die Simulationen liefern ein sehr ähnliches Beugungsverhalten wie das im Experiment durch ptychographische Rekonstruktion beobachtete, wobei die in Simulation und Rekonstruktion verwendeten Propagationsdistanzen vergleichbar sind. Das Experiment kann vollständig theoretisch beschrieben und erklärt werden. 


\section{Kapitel 5}

\section{Zusammenfassung und Ausblick}

In dieser Arbeit wurden die Entwicklung und Herstellung von Laborquellen und Optiken zur Erzeugung und Formung von Röngtenstrahlung beschrieben. Die Experimente konzentrierten sich dabei auf den Wellenlängenbereich des Wasserfensters von 2,28 nm bis $4,38 \mathrm{~nm}$ Wellenlänge.

Ein kryogenes und flüssiges Argontarget für eine Laserplasmaquelle wurde vorgestellt, die ein hochbrillant strahlendes, breitbandiges Plasma bei hoher Repetitionsrate ermöglichen soll. Die Parameter des Targets im Betrieb wurden untersucht und optimiert. Der Beleuchtungsstrahlgang eines Aufbaus zur Röntgenmikroskopie wurde justiert. Dieses Mikroskop verfolgt einen bichromatischen Ansatz und soll elementspezifische Wasserfenstermikroskopie um die Kalziumkante ermöglichen. Ein Photonenfluss von 2,9 $10^{8}$ monochromatischer Photonen von 3,45 nm Wellenlänge wurde am Ende des Beleuchtungsstrahlgangs mit einer kalibrierten XUV-Diode auf einem Beleuchtungsfleck von $60 \times 95 \mu \mathrm{m}^{2}$ pro Puls gemessen. Bei einer entsprechenden Repetitionsrate wären dies ausreichend viele Photonen für experimentell realistische Belichtungszeiten. Im Betrieb des Kryostaten zeigte sich jedoch wegen der Debris-Emission und der Lageinstabilität des Argonstrahls und seiner Operationsinstabilität im Betrieb noch weiterer Entwicklungsbedarf.

Multischicht-Laue-Linsen für weiche Röntgenstrahlung wurden in Kooperation mit dem Institut für Materialphysik der Universität Göttingen entworfen und dort in einem kombinierten Verfahren von gepulster Laserdeposition und fokussiertem Ionenstrahl hergestellt. Diese wurden mit einer Laserplasmaquelle (Ti-gefiltertes gasförmiges Stickstoffspektrum, einzelne Emissionslinie bei 2,88 nm Wellenlänge) und am BESSY II Speicherring des Helmholzzentrums in Berlin charakterisiert. Dazu wurde der Divergenzwinkel der XUV-Strahlung hinter der Linse bestimmt und die Strahltaille vermessen. Dabei wurde eine Datenauswertungsmethode vorgestellt, die aufbauend auf der Definition der Strahlbreite nach ISO11146 [1999] eine relativ robuste Auswertung von Schneidblendenrastermessungen erlaubt, auch im Fall von wenigen und verrauschten Datenpunkten. Es wurden alle relevanten Strahlparameter der Multischicht-Laue-Linse - insbesondere eine Strahltaille von $375 \pm 30 \mathrm{~nm}$ - bestimmt. Dabei lag das Verhältnis der Intensität von 
1. zu 0. Beugungsordnung bei etwa $30 \%$. Sowohl an der Laborquelle als auch am Speicherring wurde eine starke Aberration des Strahls beobachtet. Damit konnte die Ursache quellseitig ausgeschlossen und im Herstellungsprozess identifiziert werden. Umfangreiche Simulationen können in Übereinstimmung mit elektronenmikroskopischen Aufnamen die spezielle Natur der Aberration erklären.

Zur Erzeugung hoher räumlicher Kohärenz und hoch definierter Beleuchtung wurden ultra-kleine Lochblenden in dünnen Goldfolien mit fokussiertem Ionenstrahl strukturiert. Dazu wurden Vorexperimente an einer Laserplasmaquelle (Gold-Festkörpertarget) bei EUV-Strahlung von 13,5 nm Wellenlänge durchgeführt. Die Strahlung traf durch ein modifiziertes Schwarzschildobjektiv fokussiert als Hohlkegel auf die ultra-kleine Lochblende. Dabei wird bei zunehmend größer werdendem Aspektverhältnis der Lochblendentiefe zum -durchmesser die innere Apertur der Beleuchtung gefüllt. Dieser Effekt ergibt sich aus der Strahlpropagation innerhalb der tiefen ultra-kleinen Lochblende. Wellenlängenabhängige Beugungsexperimente am BESSY II Speicherring konnten die Abweichung der Strahleigenschaften vom idealen Grenzfall einer ebenen Lochblende quantifizieren. Die ultrakleinen Lochblenden werden darüber hinaus erfolgreich für ptychographische Mikroskopieaufnahmen eingesetzt. Die neben der Objektfunktion aus den Rekonstruktionen der Beugungsbilder gewonnene Beleuchtungsfunktion wurde zurück propagiert und mit dem elektronenmikroskopischen Bild des Ausgangs der Lochblende verglichen. Es zeigte sich eine gute Übereinstimmung. Ebenso ließ sich eine gute Übereinstimmung zwischen Simulationen des Wellenfelds und dem Ergebnis der ptychographischen Rekonstruktion erzielen. Damit erscheint die Beschreibung des elektromagnetischen Felds im Experiment sehr plausibel.

In weiterführenden Experimenten sollen für die Charakterisierung von Strahltaillen mit Laborquellen andere Ansätze getestet werden. So wurden bereits erste Experimente durchgeführt, die Taille der XUV-Strahlung mit Fotolacken der Elektronenstrahllithografie oder durch die Erzeugung von Farbzentren in Lithium-Fluorid-Kristallen zu detektieren. Bei letzterer Methode wurde bereits erfolgreich die Erzeugung solcher Farbzentren und deren mögliche Vermessung in der Nanometerskala durch STED-Mikroskopie in ersten Experimenten gezeigt. So könnte ein komplettes Abbild der Strahltaille in einer einzigen Belichtung registriert werden, welches den Zeitbedarf und die Genauigkeit der Strahlcharakterisierung deutlich verbessern könnte. 


\section{Anhang A}

\section{Ausbreitung eines Gaußschen Strahlenbündels}

Die Betrachtung einer sich ausbreitenden elektromagnetischen Welle als Gaußsches Strahlenbündel bietet den Zugang zu vielen relevanten Strahlparametern. Der sogenannte Gaußstrahl wird in der Literatur ausführlich beschrieben (siehe beispielsweise [Born und Wolf, 1997; Alda, 2003; Saleh und Teich, 2008]), so dass an dieser Stelle nur ein Überblick auf die in dieser Arbeit relevanten Formeln gegeben werden soll. Der Gaußstrahl ist eine Lösung der Helmholz Gleichung in paraxialer Näherung [Saleh und Teich, 2008]. Darüber hinaus seien keine Polarisationseffekte berücksichtigt und die Ausbreitung der Welle nur in einer Komponente betrachtet.

Die Amplitudenverteilung $A$ in $x$-Richtung eines sich in $z$-Richtung ausbreitenden Gaußsschen Strahls mit Wellenzahl $k=2 \pi / \lambda$ ist gegeben durch [Alda, 2003]:

$$
A(x, z)=A_{0} \sqrt{\frac{2}{\pi}} \frac{\omega_{0} e^{-i\left(\phi_{0}-\phi(z)\right)}}{\omega(z)} \cdot e^{-i \frac{k x^{2}}{2 R(z)}-\frac{x^{2}}{\omega^{2}(z)}} .
$$

$\omega_{0}$ bezeichnet die minimale Strahltaillenbreite und $\lambda$ die Wellenlänge. Die Funktionen $R(z), \omega(z)$ und $\phi(z)$ definieren das Verhalten des Strahls.

Die Weite des Strahls (und entsprechend auch die Intensität) senkrecht zur Ausbreitungsrichtung folgt der Proportionalität

$$
\begin{aligned}
& A(x, z) \propto e^{-\frac{x^{2}}{\omega^{2}(z)}} \\
& I(x, z)=|A(x, z)|^{2} \propto e^{-\frac{2 x^{2}}{\omega^{2}(z)}} .
\end{aligned}
$$

Betrachtet man die Grenze des Strahls als jene, an dem seine Intensität auf $1 / e^{2}$ abfällt, so entwickelt sich der Strahl in seiner Breite gemäß

$$
\omega(z)=\omega_{0} \sqrt{1+\left(\frac{z \lambda}{\pi \omega_{0}^{2}}\right)^{2}},
$$


wobei sein Profil an jeder Stelle die Form der Dichtefunktion der Normalverteilung hat. Diese Gleichung nähert sich für große Abstände $z$ einer Asymptote an, so dass der Divergenzwinkel im Fernfeld lautet:

$$
\theta \approx \tan (\theta)=\frac{\lambda}{\pi \omega_{0}} .
$$

Der Krümmungsradius der Wellenfront des Gaußstrahls ist durch den Imaginärteil der Exponentialfunktion in Gleichung (A.1) gegeben. Er folgt:

$$
R(z)=z\left(1+\left(\frac{\pi \omega_{0}^{2}}{z \lambda}\right)^{2}\right) .
$$

Alle drei dieser Parameter werden von $\omega_{0}$ und $\lambda$ charakterisiert. Somit definiert man die für einen Gaußschen Strahl charakteristische Rayleighlänge:

$$
z_{R}=\frac{\pi \omega_{0}^{2}}{\lambda}
$$

Der letzte Phasenterm in Gleichung (A.1) drückt die Guoy Phase aus, eine Phasenänderung an der Strahltaille, die einen Sprung um $\pi$ bewirkt (verglichen mit einer ebenen Welle, die die gleiche Strecke durchläuft):

$$
\phi(z)=\arctan \left(\frac{z}{z_{R}}\right) .
$$




\section{Anhang B}

\section{Übertragung der Momentenbreite in Halbwertsbreite bei einer Gaußschen Normalverteilung}

Die im Schrifttum häufig zu findenden Angaben einer Halbwertsbreite oder FWHM (engl. full width half maximum) können, im Falle eines Strahls, dessen Profil der Gaußschen Normalverteilungsfunktion folgt, mittels eines linearen Zusammenhangs in den $1 / e^{2}$-Abfall umgerechnet werden. Das ist insbesondere wichtig, da dieser Abfall bei einem solchen Strahlprofil identisch mit dem zweiten Bildmoment ist. Dadurch lassen sich die Ergebnisse der Momentenauswertung in dieser gebräuchlichen Konvention darstellen. Das Intensitätsprofil $I$ eines Gauß'schen Strahls folgt:

$$
I=I_{0} \cdot e^{-2 \frac{x^{2}}{w^{2}}}
$$

Hierbei bezeichnet $x$ den Abstand von der Strahlmitte und $w^{2}$ die halbe Strahlbreite am $1 / e^{2}$-Abfall, also die doppelte Varianz $\sigma^{2}$ der zugehörigen Normalverteilungsfunktion. Daher kann der Abfall auf die Hälfte der maximalen Strahlintensität ausgedrückt werden durch:

$$
\begin{aligned}
\frac{1}{2} \cdot I_{0} & =I_{0} \cdot e^{-2 \frac{\left(\frac{F W H M}{2}\right)^{2}}{w^{2}}} \\
\Rightarrow-\frac{1}{2} \cdot \frac{F W H M^{2}}{w^{2}} & =\ln \left(\frac{1}{2}\right)=-\ln (2) \\
\Rightarrow \quad F W H M^{2} & =2 \cdot \ln (2) \cdot w^{2} \\
\Rightarrow \quad F W H M & =\sqrt{2 \cdot \ln (2)} \cdot w \\
& \approx 1,386 \cdot w .
\end{aligned}
$$




\section{Anhang C}

\section{Simulation der Experimente mit Multischicht-Laue-Linsen}

Das Programm zur Simulation der Experimente mit den Multischicht Laue Linsen ist im Folgenden dargestellt. Wichtige Schritte sind durch Kommentare im Quelltext hervorgehoben. Das Programm gliedert sich in zwei Teile, erstens der Berechnung des Wellenfeldes hinter der MLL und zweitens der Auswertung und Darstellung der Berechnung.

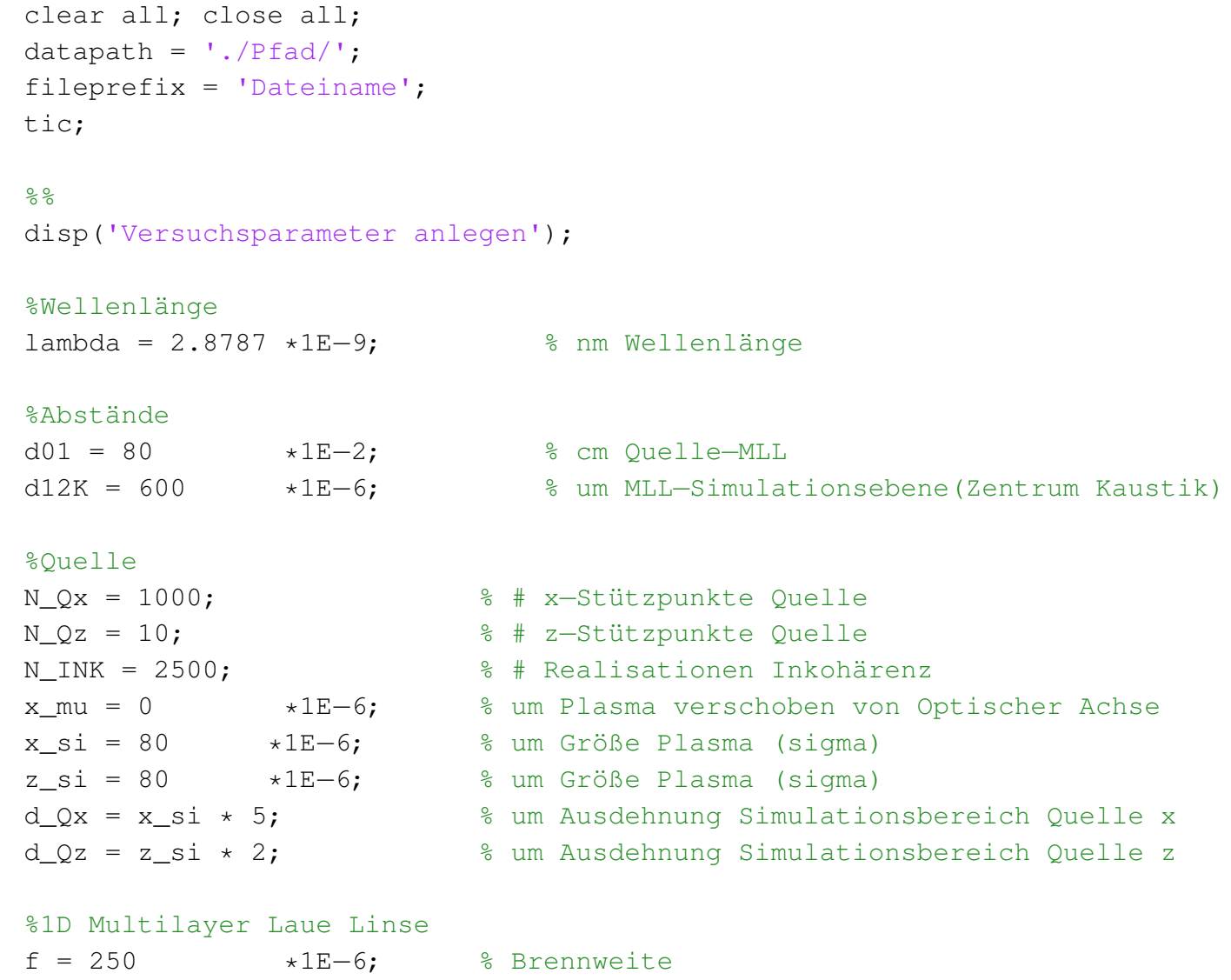




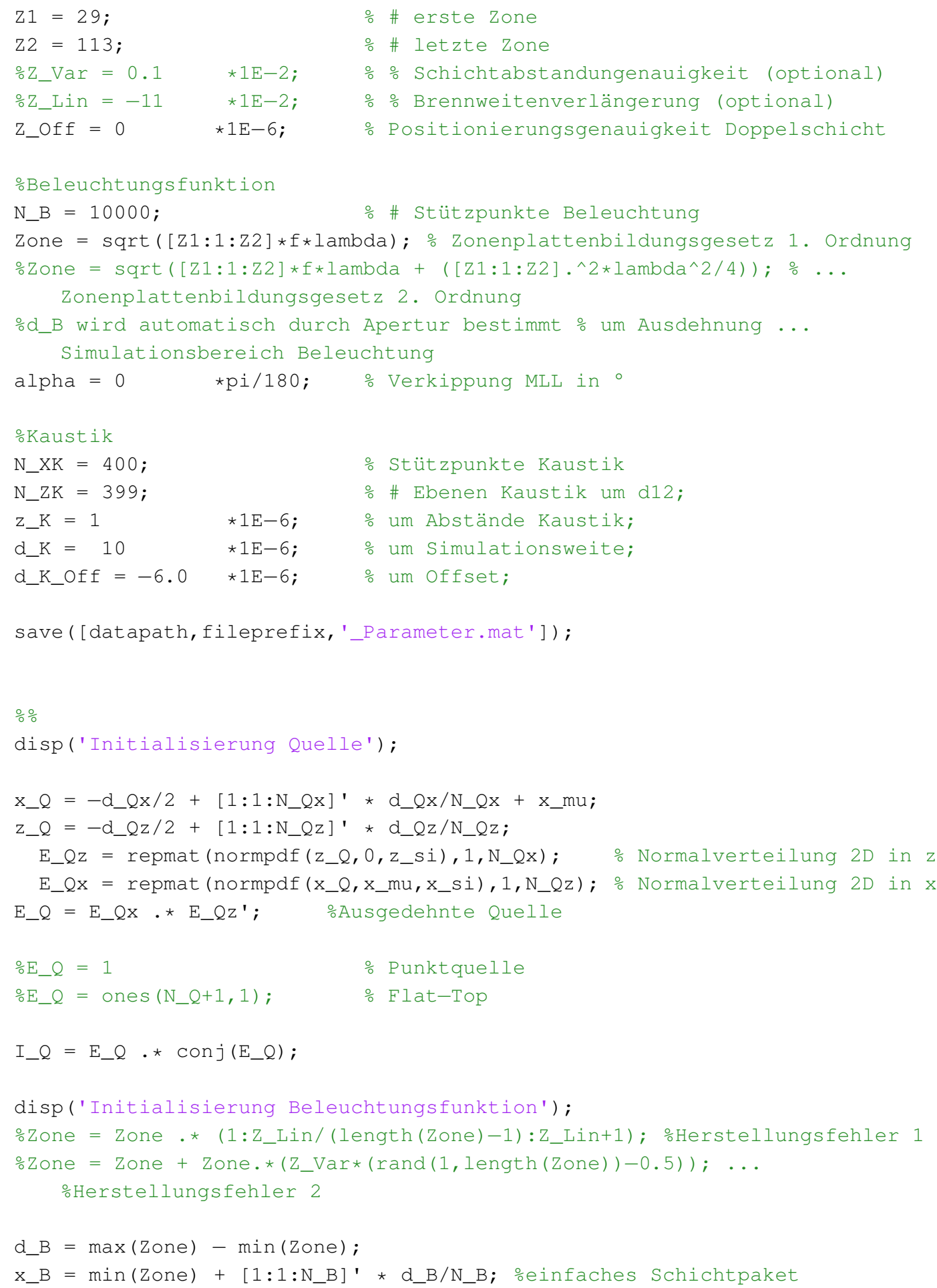




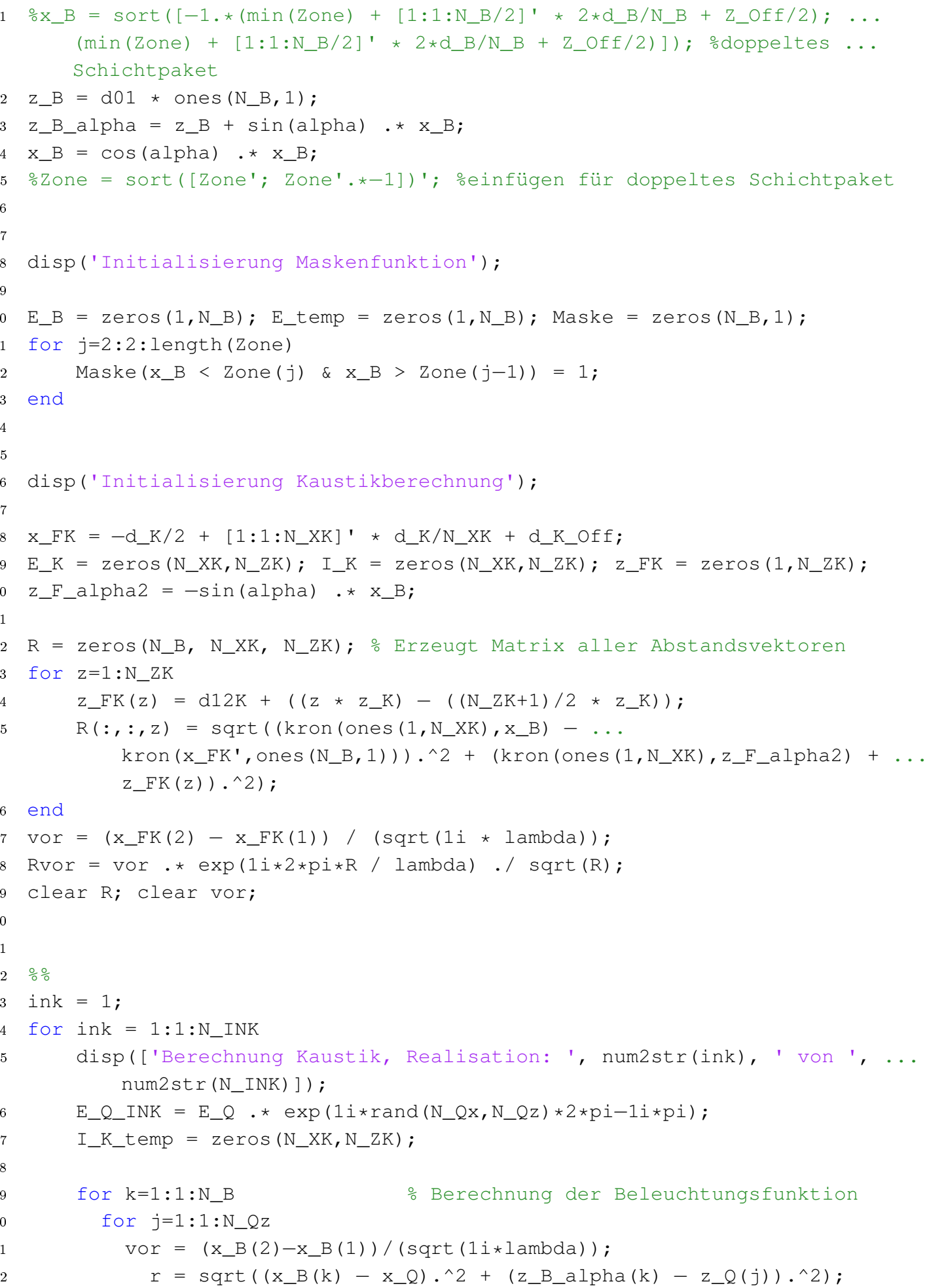




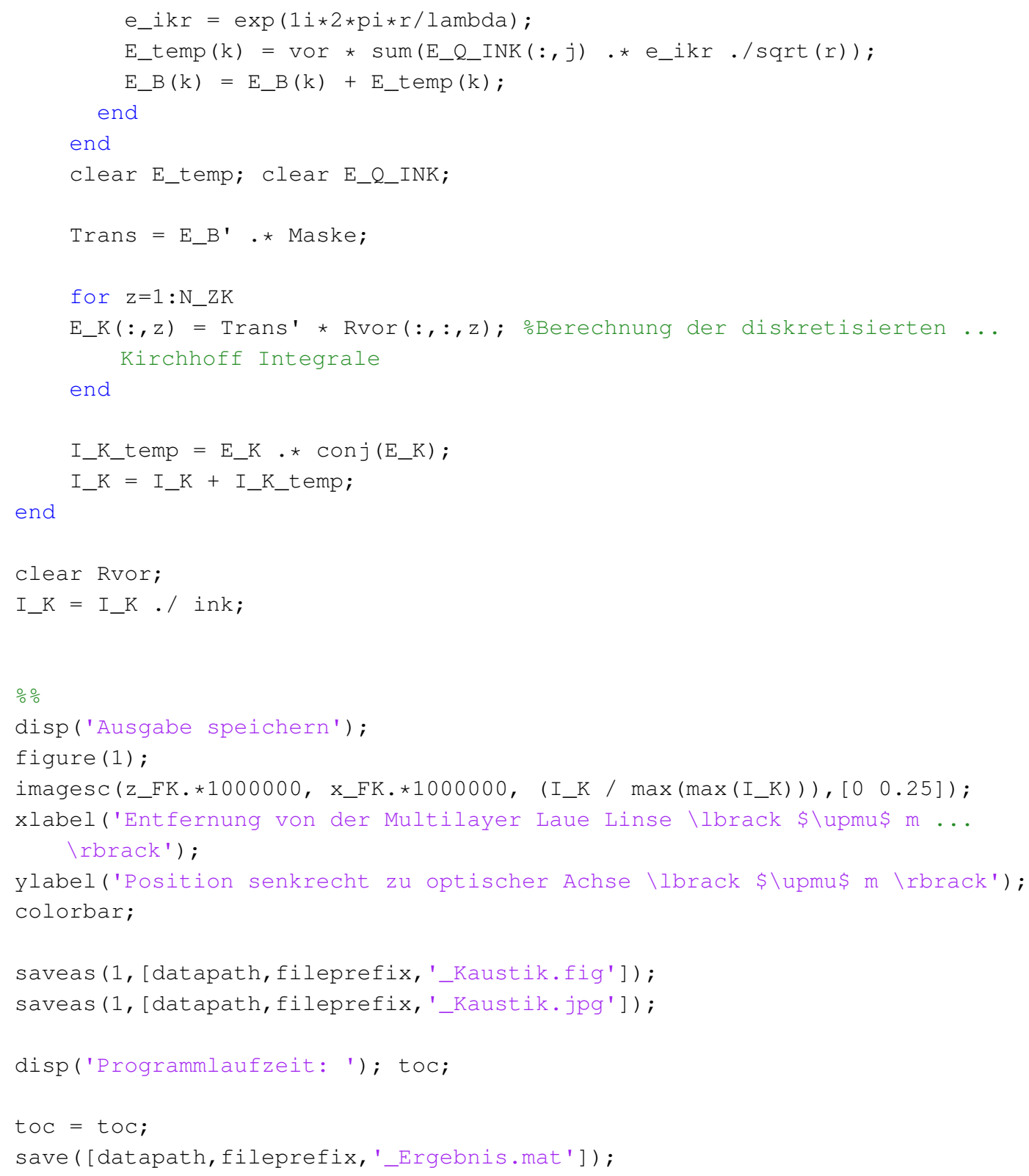


Es folgt der zweite Programmteil zur Analyse der simulierten Felder:

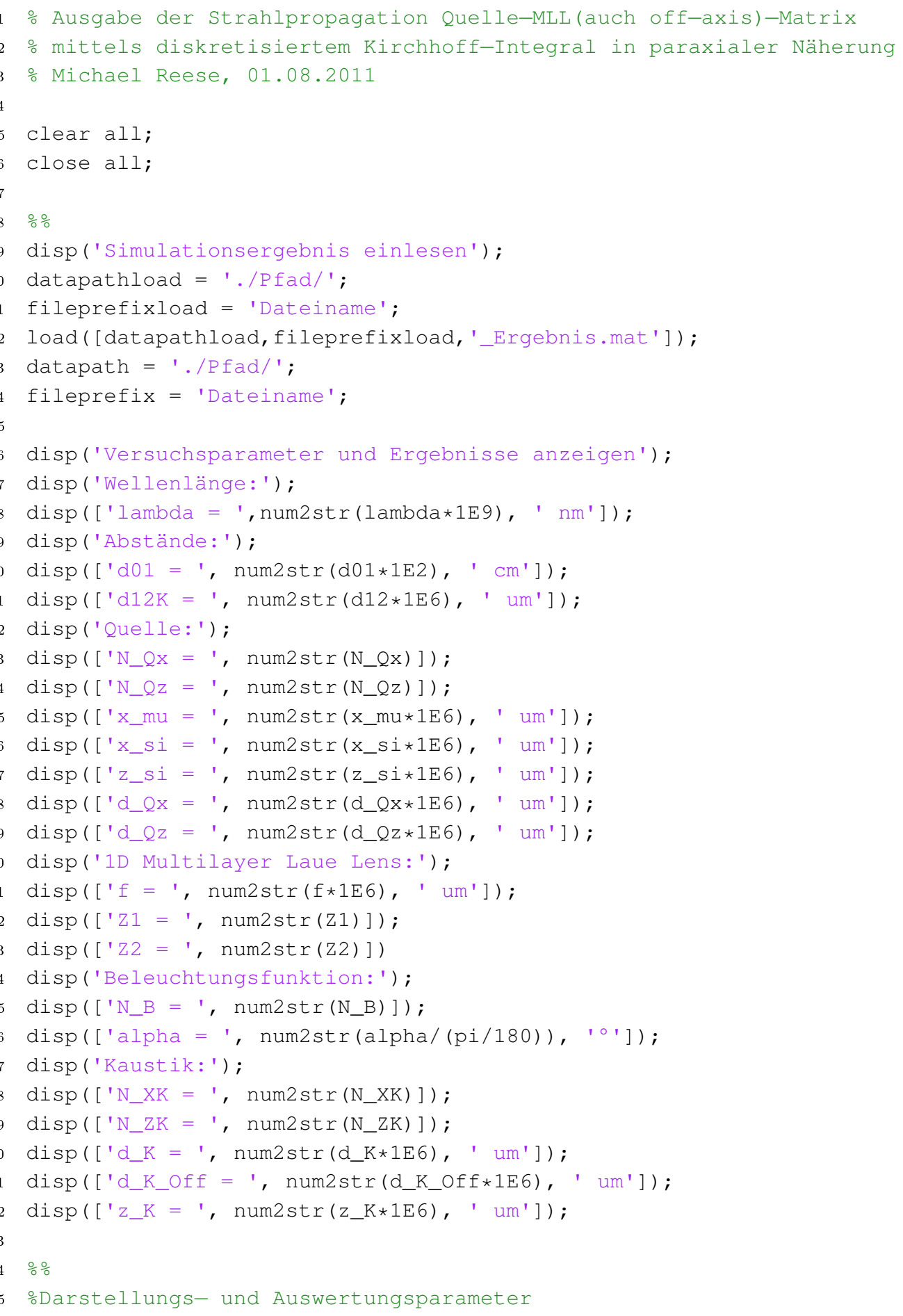




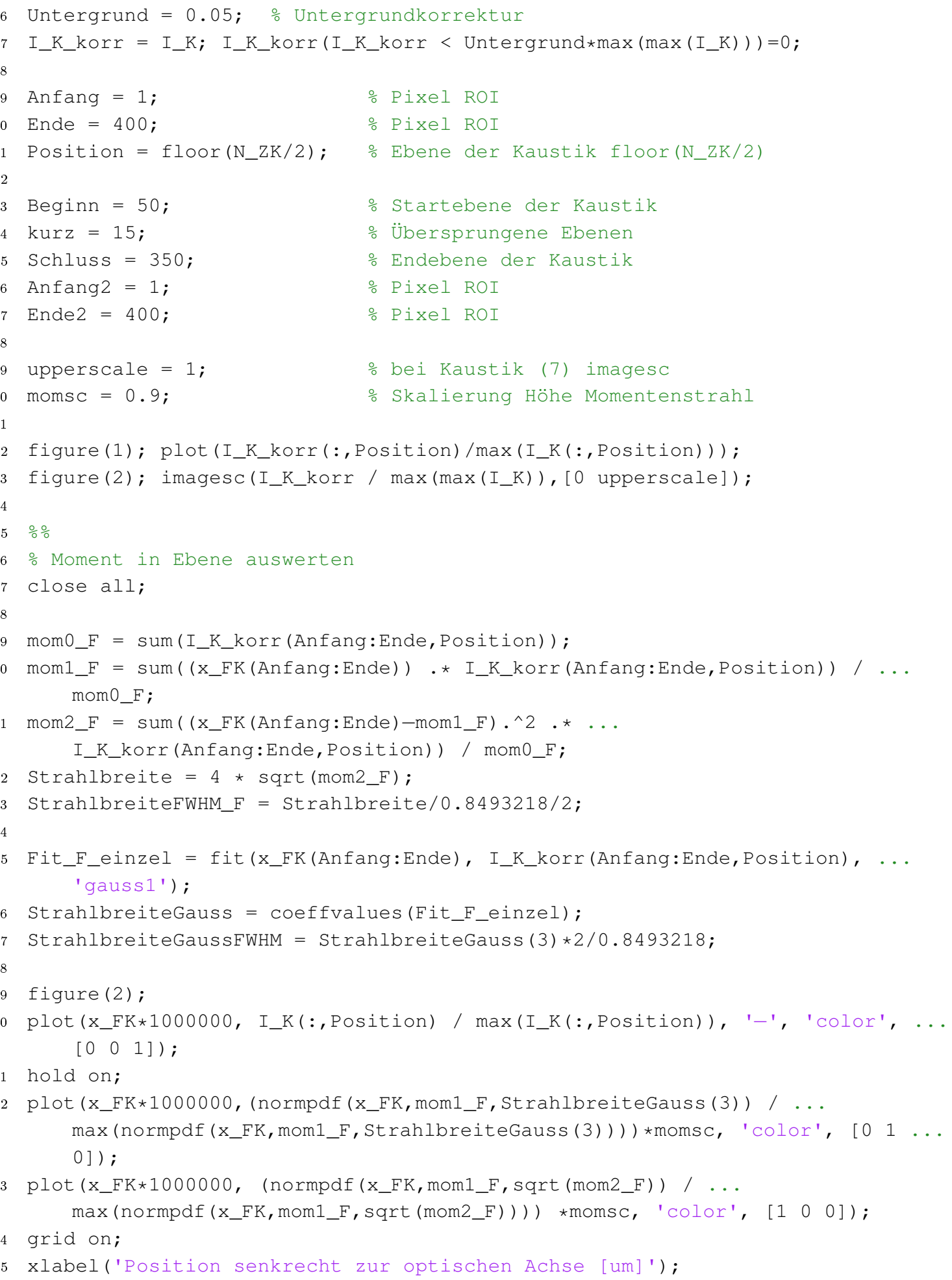




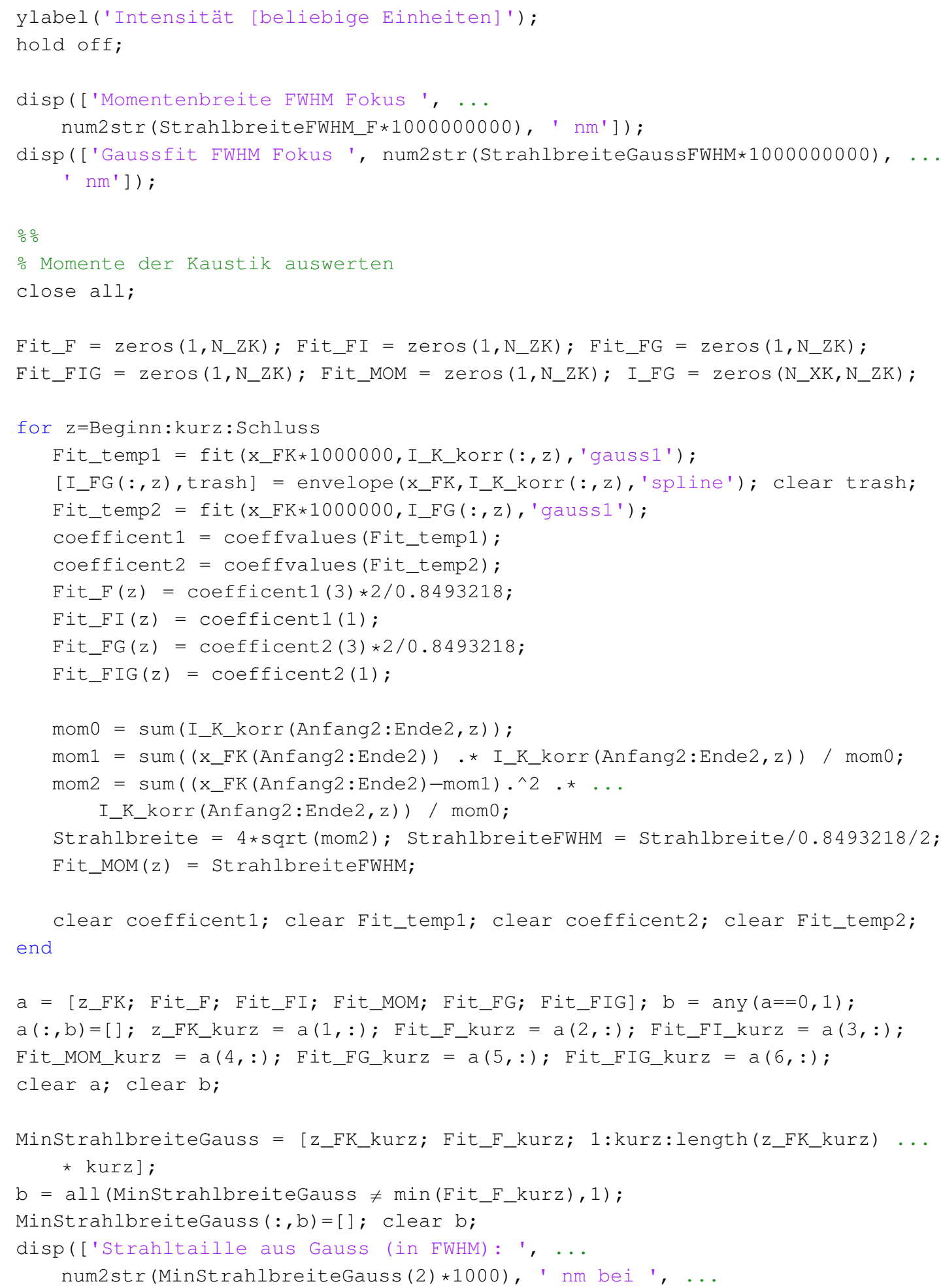




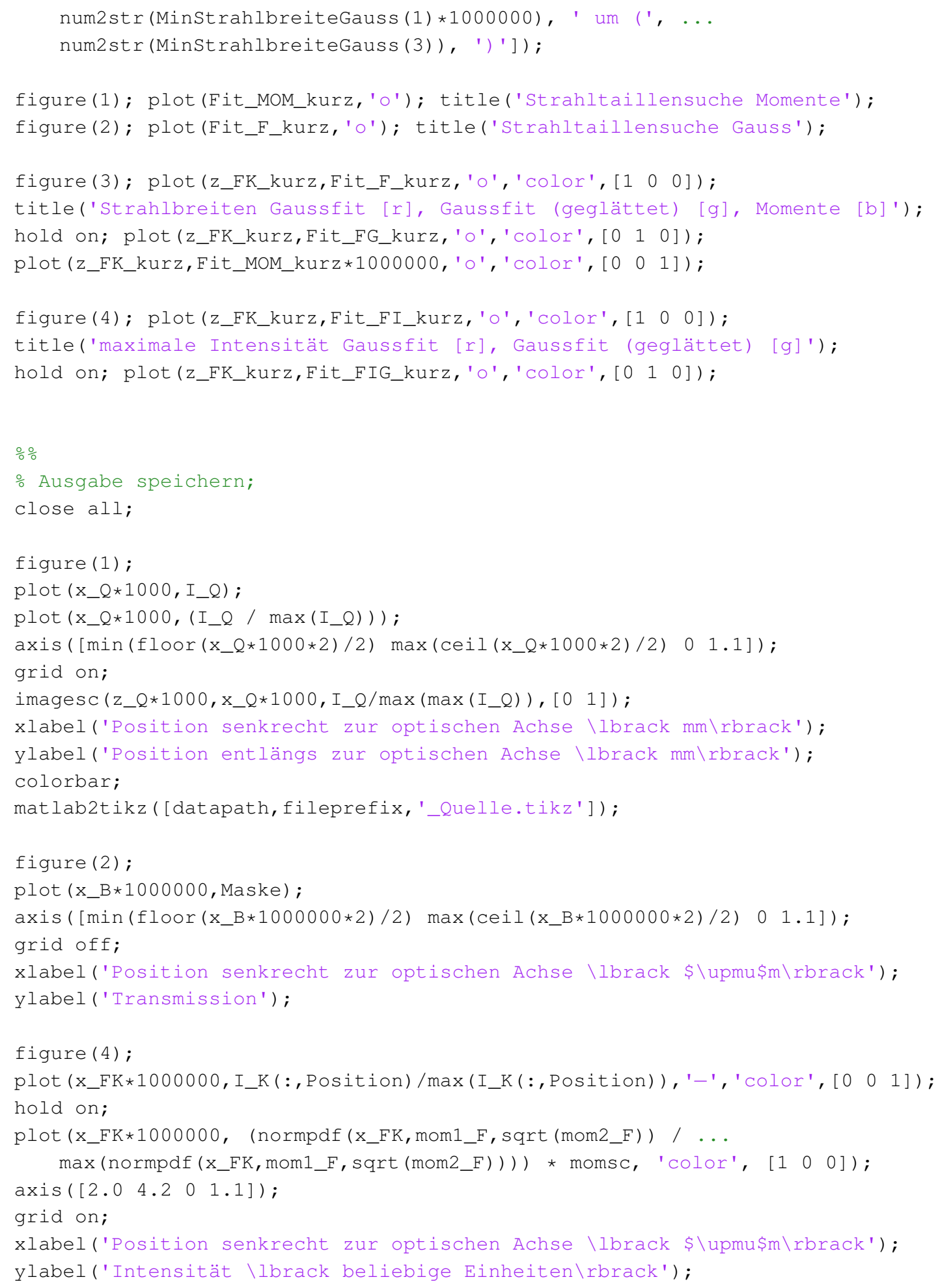


172

173

174

figure (5);

imagesc(z_FK. $\star 1000000$, X_FK. $\star 1000000$, (I_K / $\left.\max \left(\max \left(I \_K\right)\right)\right),[0$ upperscale]); xlabel('Position senkrecht zur optischen Achse \lbrack \$ \upmu\$m\rbrack'); ylabel('Intensität \lbrack beliebige Einheiten\rbrack');

colorbar;

matlab2tikz([datapath,fileprefix,'_Kaustik.tikz']) ;

figure (6);

plot (z_FK_kurz*1000000,Fit_F_kurz*1000,' ' ');

grid on;

xlabel('Entfernung von der Multilayer Laue Linse \lbrack \$ \upmu\$m\rbrack'); ylabel ('Strahlbreite in FWHM \lbrack nm\rbrack');

$\operatorname{axis}\left(\left[\min \left(f l o o r\left(z \_F K \_k u r z * 1000000 / 1\right)\right) * 1 \max \left(\right.\right.\right.$ ceil $\left.\left(z \_F K \_k u r z * 1000000 / 1\right) * 1\right) \ldots$ $100 \max ($ ceil (Fit_F_kurz*1000/100)*100)]);

figure (7);

plot (z_FK_kurz*1000000,Fit_MOM_kurz $\left.\star 1000000000, \mathrm{O}^{\prime}\right)$;

grid on;

xlabel('Entfernung von der Multilayer Laue Linse \lbrack \$\upmu\$m\rbrack');

ylabel('Strahlbreite in FWHM \lbrack nm\rbrack');

axis ([min(floor (z_FK_kurz*1000000/1)) *1 max(ceil(z_FK_kurz*1000000/1)*1) .. 400 max (ceil(Fit_MOM_kurz $* 1000000000 / 100) * 100)])$;

saveas (1, [datapath, fileprefix, '_Quelle.jpg']);

saveas (2, [datapath,fileprefix, '_Maskenfunktion.jpg']) ;

saveas (4, [datapath, fileprefix, '_Fokusfunktion_ausKaustik.jpg']) ;

saveas (5, [datapath, fileprefix, '_Kaustik.jpg']) ;

saveas (6, [datapath, fileprefix,'_StrahlbreiteGauss.jpg']);

saveas (7, [datapath,fileprefix, '_StrahlbreiteMom.jpg']) ;

Fokus $=\left[\mathrm{X} \_F K\right.$, ones $\left.\left(\mathrm{N} \_\mathrm{XK}, 1\right) . * \mathrm{~d} 12, \mathrm{I} \_\mathrm{K}, \mathrm{E} \_\mathrm{K}\right]$;

Strahlbreiten = [z_FK_kurz, Fit_F_kurz, Fit_MOM_kurz $]$;

xlswrite([datapath,fileprefix, '_Fokusfunktion.xls'], Fokus);

xlswrite([datapath, fileprefix, '_Strahlbreiten.xls'], Strahlbreiten);

matfig2pgf('filename', [datapath,fileprefix, '_Maskenfunktion.pgf'], ...

'fignr', 2, 'converttexttolatex', false, 'figwidth', 12);

matfig2pgf('filename', ...

[datapath, fileprefix, '_Fokusfunktion_auskaustik.pgf'], 'fignr', 4, ...

'converttexttolatex', false, 'figwidth', 12);

matfig2pgf('filename', [datapath,fileprefix, '_StrahlbreiteGauss.pgf'], ...

'fignr', 6, 'converttexttolatex', false, 'figwidth', 12);

matfig2pgf('filename', [datapath,fileprefix,'_StrahlbreiteMom.pgf'], ...

'fignr', 7, 'converttexttolatex', false, 'figwidth', 12); 
disp(['Momentenbreite FWHM Fokus: ', ... num2str(StrahlbreiteFWHM_F*1000000000), ' nm']); 


\section{Anhang D}

\section{Analyse der Minima und Maxima von Beugungsbildern ultra-kleiner Lochblenden}

Das Programm zur Analyse der Minima (und Maxima) von Beugungsbildern ultrakleiner Lochblenden ist im Folgenden angegeben. Wichtige Schritte sind durch Kommentare im Quelltext hervorgehoben.

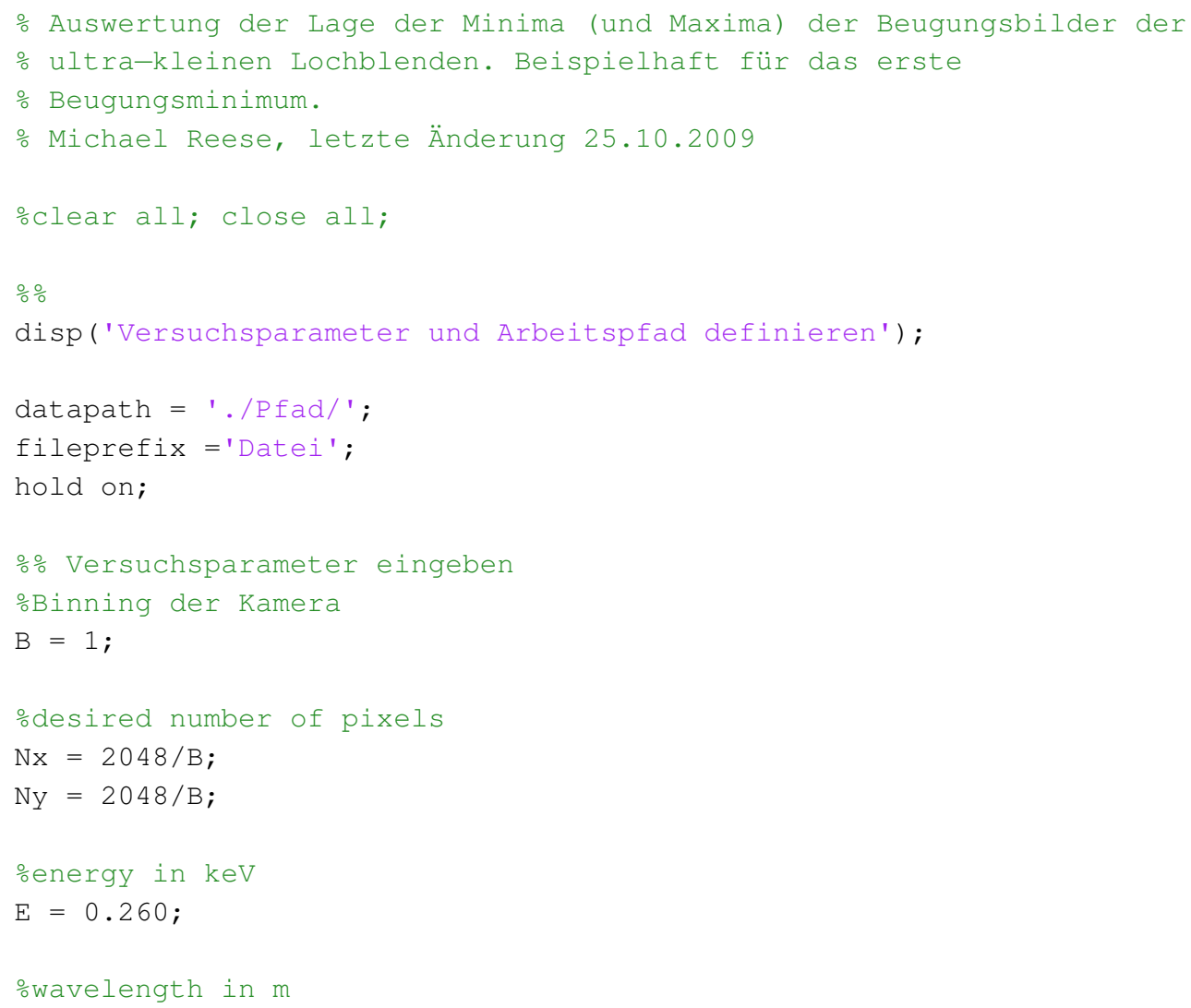




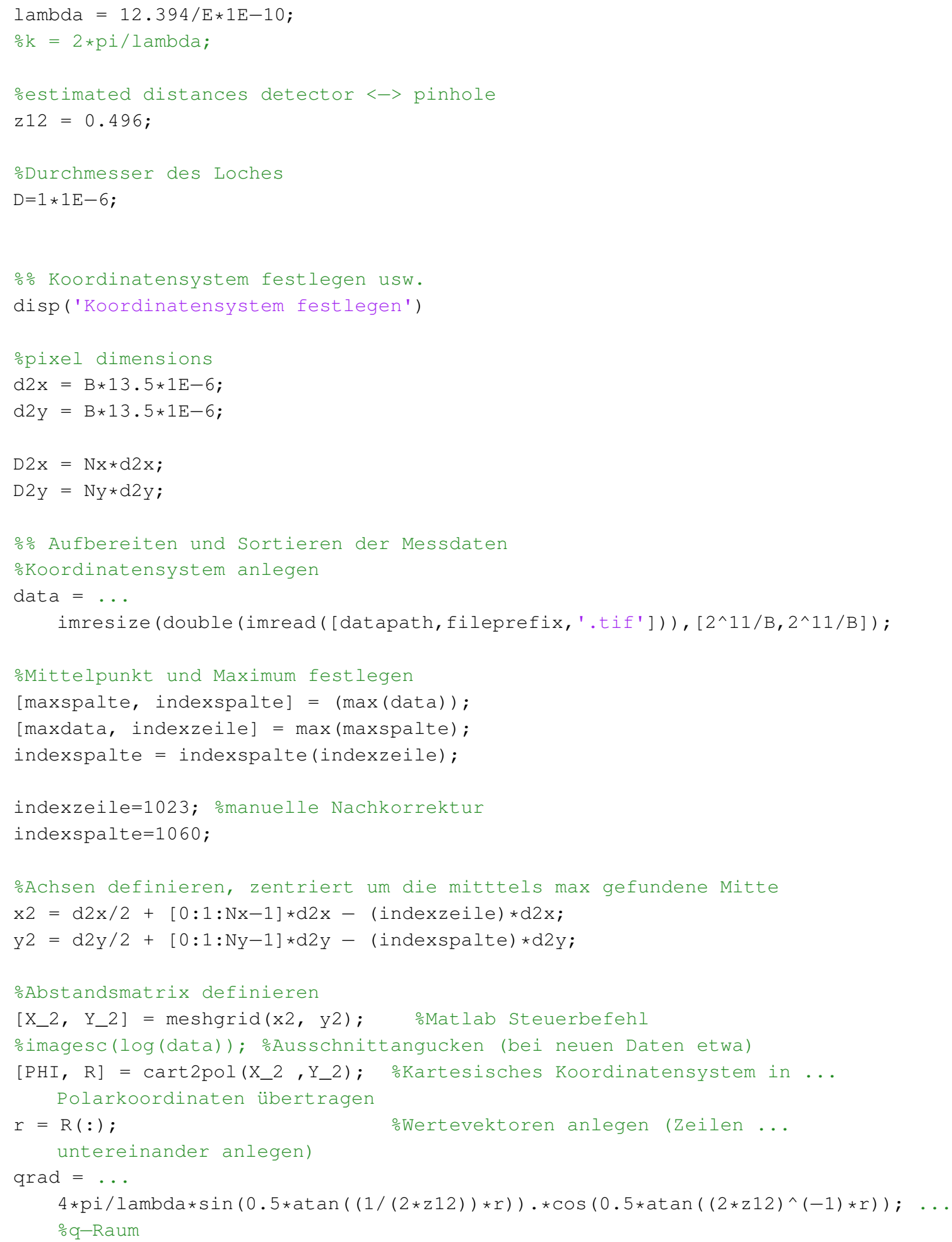


69 phi = PHI (:);

$70 \mathrm{phi}=\operatorname{round}(70 * \mathrm{phi}) / 70$; zusammenfassbar werden)

$71 \%$ round $(10000 * r) / 10000$;

72 clear PHI; clear R;

73 


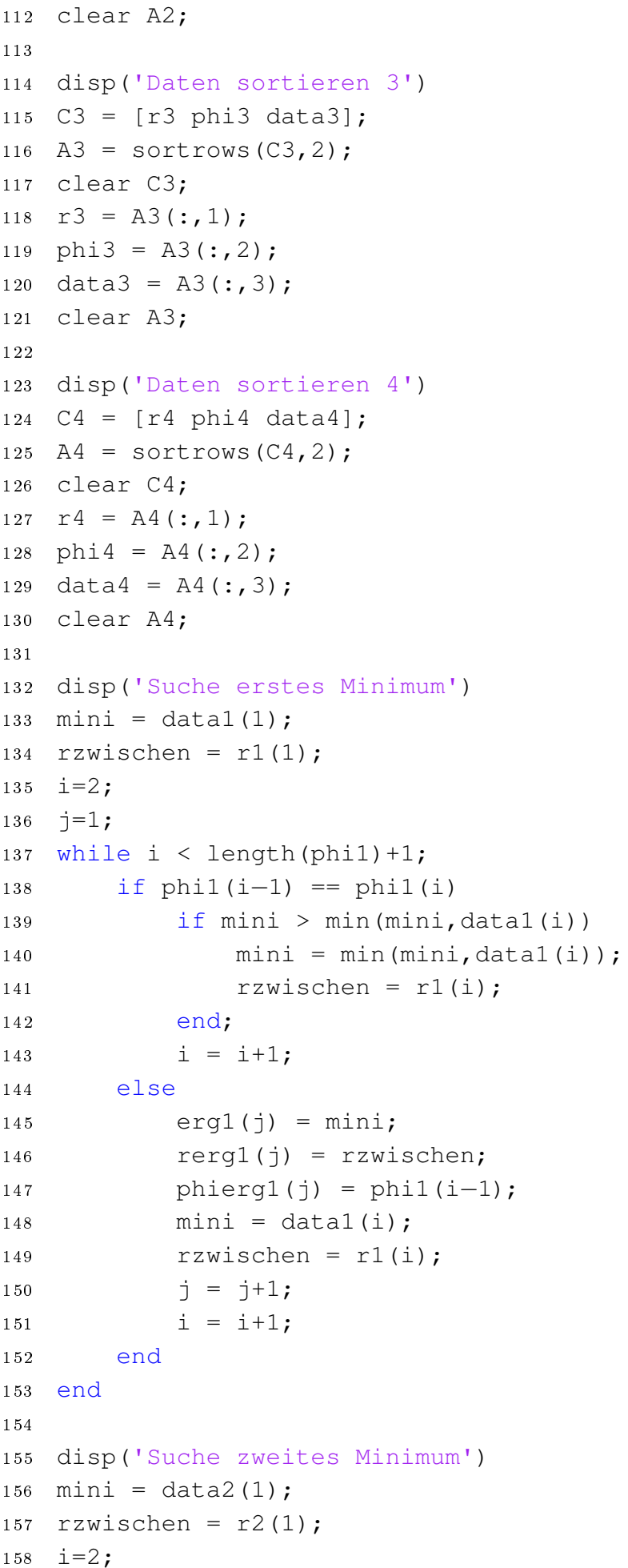




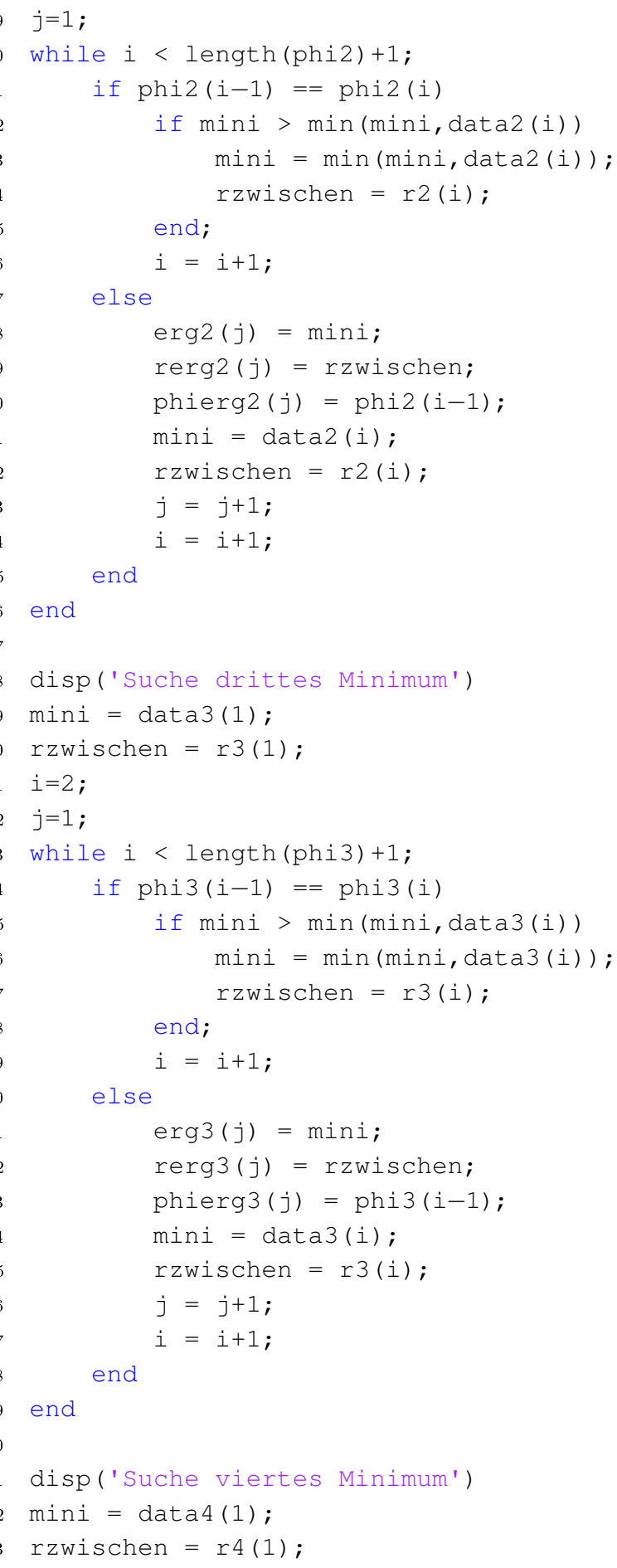




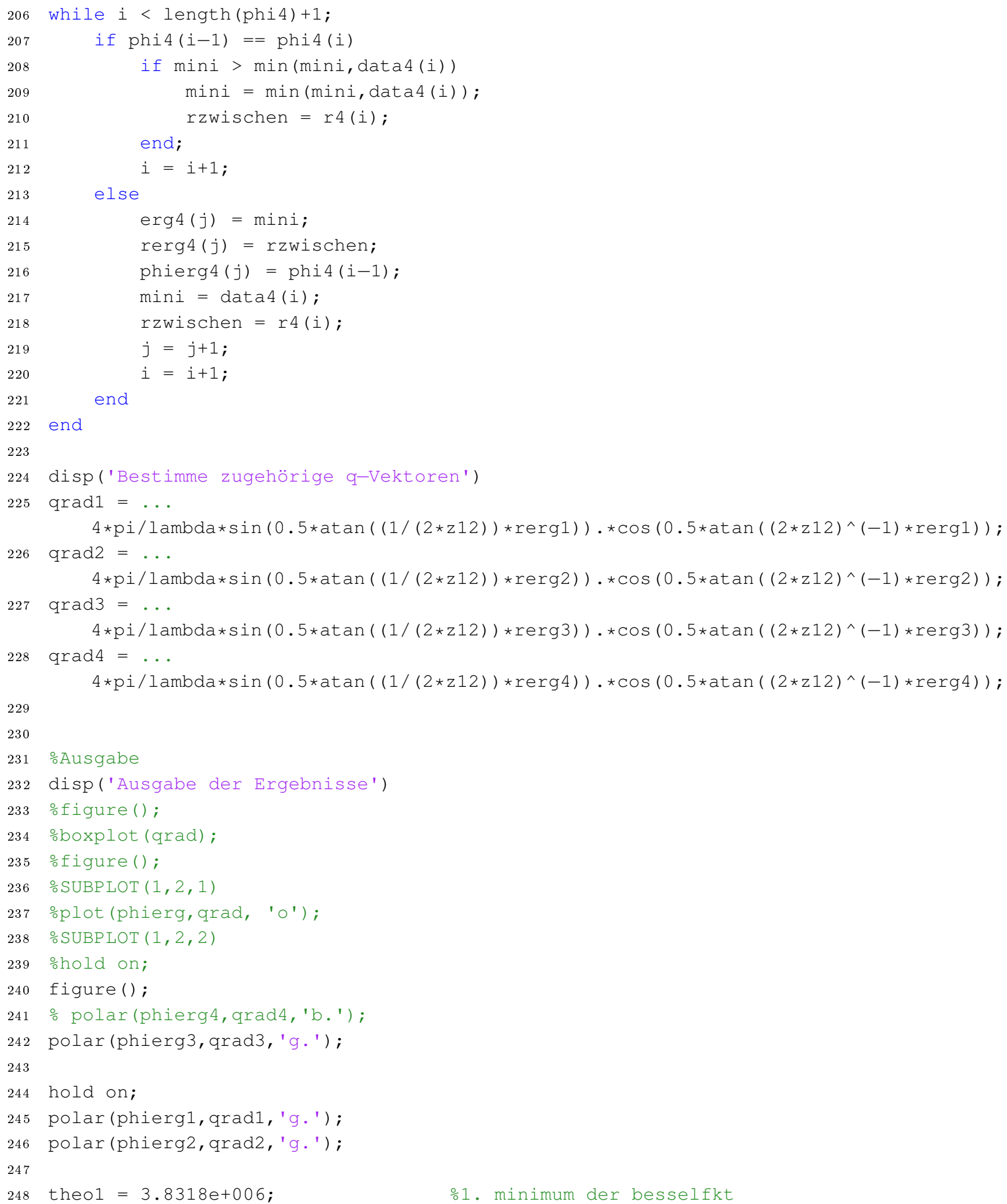


249 kreis1 = phierg1 $10+1$;

250 kreisl = theol $*$ kreis 1 ;

251 theo2 $=7.0154$ e+006;

252 kreis2 $=$ phierg $2 \star 0+1$;

253 kreis2 $=$ theo $2 *$ kreis 2 ;

254 theo3 $=1.0173 e+007$;

255 kreis $3=$ phierg $3 * 0+1$;

256 kreis3 $=$ theo $3 *$ kreis 3 ;

257 theo4 $=1.3323 e+007$;

258 kreis4 $=\operatorname{phierg} 4 * 0+1$;

259 kreis4 $=$ theo $4 *$ kreis 4 ;

260 hold on;

261 set (polar (phierg1,kreis1, 'red-'), 'Linewidth', 2.2);

262 set (polar(phierg2,kreis2, 'red-'), 'Linewidth', 2.2) ;

263 set (polar (phierg3,kreis3, 'red_' '), 'LineWidth', 2.2);

264 set(polar (phierg4,kreis4,'r'), 'LineWidth', 2.2) ;

265

266

267 disp('Anzahl der Datenpunkte pro Auswertungswinkel')

268 anzahlprophi1 = length (data1)/length (erg1)

269 anzahlprophi2 = length (data2)/length (erg2)

270 anzahlprophi3 = length (data3)/length (erg3)

271 anzahlprophi4 = length (data4)/length (erg4)

272 hold off; 



\section{Literaturverzeichnis}

[Abramoff u. a. 2004] Abramoff, M. D. ; Magelhaes, P. J. ; Ram, S. J.: Image Processing with ImageJ. In: Biophotonics International 11 (2004), S. 36-42

[Ackermann u. a. 2007] Ackermann, W. ; Asova, G. ; Ayvazyan, V. ; Azima, A. ; Baboi, N. ; Bahr, J. ; Balandin, V. ; Beutner, B. ; Brandt, A. ; Bolzmann, A. ; Brinkmann, R. ; Brovko, I. O. ; Castellano, M. ; Castro, P. ; Catani, L. ; Chiadroni, E. ; Choroba, S. ; Cianchi, A. ; Costello, J. T. ; Cubaynes, D. ; Dardis, J. ; Decking, W. ; Delsim-Hashemi, H. ; Delserieys, A. ; DiPirro, G. ; Dohlus, M. ; Dusterer, S. ; Eckhardt, A. ; H. T., Edwards ; Faatz, B. ; Feldhaus, J. ; Flottmann, K. ; Frisch, J. ; Frohlich, L. ; Garvey, T. ; Gensch, U. ; Gerth, Ch. ; Gorler, M. ; Golubeva, N. ; Grabosch, H.-J. ; Grecki, M. ; Grimm, O. ; Hacker, K. ; Hahn, H. U. ; Han, J. ; Honkavaara, K. ; Hott, H. T. ; Huning, M. ; Ivanisenko, Y. ; Jaeschke, E. ; Jalmuzna, W. ; Jezynski, T. ; Kammering, R. ; Katalev, V. ; Kavanagh, T. K. ; Kennedy, E. ; Khodyachykh, S. ; Klose, K. ; Kocharyan, V. ; Korfer, M. ; Kollewe, M. ; Koprek, W. ; Korepanov, S. ; Kostin, D. ; Krassilnikov, M. ; Kube, G. ; Kuhlmann, M. ; S., C. L. ; Lilje, L. ; Limberg, T. ; Lipka, D. ; Lohl, F. ; LunA, H. ; Luong, M. ; Martins, M. ; Meyer, M. ; Michelato, P. ; Miltchev, V. ; Moller, W. D. ; Monaco, L. ; Muller, W. F. O. ; Napieralski, O. ; Napoly, O. ; Nicolosi, P. ; Nolle, D. ; Nunez, T. ; Oppelt, A. ; Pagani, C. ; Paparella, R. ; Pchalek, N. ; Pedregosa-Gutierrez, J. ; Petersen, B. ; Petrosyan, B. ; Petrosyan, G. ; Petrosyan, J. ; Pfluger, J. ; Plonjes, E. ; Poletto, L. ; Pozniak, K. ; Pra, te. ; Proch, D. ; Pucyk, P. ; Radcliffe, P. ; Redlin, H. ; Rehlich, K. ; Richter, M. ; Roehrs, M. ; Roensch, J. ; Romaniuk, R. ; Ross, M. ; Rossbach, J. ; Rybnikov, V. ; Sachwitz, M. ; L., E. ; Sandner, W. ; Schlarb, H. ; Schmidt, B. ; Schmitz, M. ; Schmuser, P. ; Schneider, J. R. ; Schneidmiller, E. A. ; Schnepp, S. ; Schreiber, S. ; Seidel, M. ; Sertore, D. ; Shabunov, A. V. ; Simon, C. ; Simrock, S. ; Sombrowski, E. ; Sorokin, A. A. ; Spanknebel, P. ; Spesyvtsev, R. ; Staykov, L. ; Steffen, B. ; Stephan, F. ; Stulle, F. ; Thom, H. ; Tiedtke, K. ; Tischer, M. ; Toleikis, S. ; Treusch, R. ; Trines, D. ; Tsakov, I. ; Vogel, E. ; Weiland, T. ; Weise, H. ; Wellhofer, M. ; Wendt, M. ; Will, I. ; Winter, A. ; Wittenburg, K. ; Wurth, W. ; Yeates, P. ; Yurkov, M. V. ; 
Zagorodnov, I. ; Zapfe, K.: Operation of a free-electron laser from the extreme ultraviolet to the water window. In: Nature Photonics 1 (2007), S. 336 - 342. - URL http://dx.doi.org/10.1038/nphoton.2007.76. - ISSN 1749-4885

[Alda 2003] ALDA, Javier: Laser and Gaussian Beam Propagation and Transformation. In: Encyclopedia of Optical Engineering (2003), S. 999 - 1013

[Alianelli u. a. 2011] Alianelli, L. ; Sawhney, K. J. S. ; Barrett, R. ; Pape, I. ; Malik, A. ; Wilson, M. C.: High efficiency nano-focusing kinoform optics for synchrotron radiation. In: Optics Express 19 (2011), S. 11120 - 11127

[Als-Nielsen und McMorrow 2001] Als-Nielsen, Jens; McMorrow, Des: Elements of Modern X-Ray Physics. John Wiley \& Sons, Ltd., 2001

[Amano u. a. 2010] Amano, Sho ; Inaoka, Yutaka ; Hiraishi, Hiroki ; MiYamoto, Shuji ; Mochizuki, Takayasu: Laser-plasma debris from a rotating cryogenic-solid-Xe target. In: Review of Scientific Instruments 81 (2010), S. 023104

[Attwood 1999] AtTwood, David: Soft X-Rays and Extreme Ultraviolet Radiation: Principles and Applications. Cambridge University Press, 1999

[Barkusky 2010] BARKUSKY, Frank: Erzeugung intensiver EUV-Strahlung und deren Wechselwirkung mit Materie, Universität Göttingen, Dissertation, 2010

[Barkusky u. a. 2007] Barkusky, Frank ; Peth, Christian ; Bayer, Armin ; Mann, Klaus: Direct photo-etching of poly(methyl methacrylate) using focused extreme ultraviolet radiation from a table-top laser-induced plasma source. In: Journal of Applied Physics 101 (2007), S. 124908

[Barth u. a. 2010] Barth, Ruth; Staier, Florian ; Simpson, Todd ; Mittler, Silvia ; Eisebitt, Stefan ; Grunze, Michael ; Rosenhahn, Axel: Soft X-ray holographic microscopy of chromosomes with high aspect ratio pinholes. In: Journal of Biotechnology (2010), S. $238-242$

[Bearden und Burr 1967] Bearden, J. A. ; Burr, A. F.: Reevaluation Of X-Ray Atomic Energy Levels. In: Review of Modern Physics 1 (1967), S. 125

[Beckers u. a. 2011] Beckers, Mike ; Senkbeil, Tobias ; Gorniak, Thomas ; Reese, Michael ; Giewekemeyer, Klaus ; Gleber, Sophie-Charlotte ; Salditt, Tim ; Rosenhahn, Axel: Chemical contrast in soft x-ray ptychography. In: Physical Review Letters 107 (2011), S. 208101 
[Benk u. a. 2008] Benk, Markus ; Bergmann, Klaus ; Schäfer, David ; Wilhein, Thomas: Compact soft x-ray microscope using a gas-discharge light source. In: Optics Letters 33 (2008), S. $2359-2361$

[Bennett 1934] Bennett, W. H.: Magnetically self-focussing streams. In: Physical Review 45 (1934), S. $0890-0897$

[Bergemann u. a. 2003] Bergemann, C. ; Keymeulen, H. ; Veen, J. F. van der: Focusing X-Ray Beams to Nanometer Dimensions. In: Physical Review Letters 91 (2003), S. 204801

[Berger u. a. 2010] Berger, M. J. ; Hubbell, J. H. ; Seltzer, S.M. ; Chang, J. ; Coursey, J. S. ; Sukumar, R. ; Zucker, D. S. ; Olsen, K.: XCOM: Photon Cross Sections Database. November 2010. - URL http://www.nist.gov/pml/data/xcom/ index.cfm

[Berglund u.a. 2000] Berglund, M. ; Rymell, L. ; Peuker, M. ; Wilhein, T. ; Hertz, H. M.: Compact water-window transmission X-ray microscopy. In: Journal of Microscopy 197 (2000), S. $268-273$

[Bergmann u.a. 2008] Bergmann, Klaus ; Küpper, Felix ; Benk, Markus: Soft x-ray emission from a pulsed gas discharge in a pseudosparklike electrode geometry. In: Applied Physics 103 (2008), S. 123304

[Bertilson u.a. 2011] Bertilson, Michael ; Hofsten, Olov von ; Vogt, Ulrich ; Holmberg, Anders ; Christakou, Athanasia E. ; Hertz, Hans M.: Laboratory soft-x-ray microscope for cryotomography of biological specimens. In: Optics Letters 36 (2011), S. $2728-2730$

[Bethe 1944] Bethe, H. A.: Theory of Diffraction by Small Holes. In: The Physical Review 66 (1944), S. $163-182$

[Bilderback und Thiel 1995] Bilderback, D. H. ; Thiel, D. J.: Microbeam Generation With Capillary Optics. In: Review of Scientific Instruments 44 (1995), S. 2059 $-2063$

[Birch u. a. 2003] Birch, Jens ; ERIKsson, Fredrik ; Johansson, Göran A. ; HerTz, Hans M.: Recent advances in ion-assisted growth of $\mathrm{Cr} / \mathrm{Sc}$ multilayer X-ray mirrors for the water window. In: Vacuum 68 (2003), S. $275-282$

[Bonfigli u. a. 2004] Bonfigli, Francesca ; Baldacchini, G. ; Faenov, A. ; Flora, F. ; Lai, A. ; Marolo, T. ; Montereali, R.M. ; Nichelatti, E. ; Pikuz, T. ; 
REALE, L.: Miniaturized optical sources and imaging detectors based on soft Xray irradiated $\mathrm{LiF}$ material. In: 7th International EULASNET meeting Workshop "Umbrella for laser and optical technologies" ENEA, UTS Tecnologie Fisiche Avanzate, Solid State Laser and Spectroscopy Laboratory (Veranst.), 2004

[Born und Wolf 1997] Born, Max ; Wolf, Emil: Principles of Optics: Electromagnetic Theory of Propagation, Interference and Diffraction of Light, 6th edition. Cambridge University Press, 1997

[Chao u. a. 2003] Chao, Weilun ; Anderson, Erik ; Denbeaux, Gregory P. ; Harteneck, Bruce ; Liddle, J. A. ; Olynick, Deirdre L. ; Pearson, Angelic L. ; Salmassi, Farhad ; Song, Cheng Y. ; Attwood, David T.: 20-nm-resolution soft $\mathrm{x}$-ray microscopy demonstrated by use of multilayer test structures. In: Optics Letters 28 (2003), S. $2019-2021$

[Chao u. a. 2005] ChaO, Weilun ; Harteneck, Bruce ; Liddle, Alexander ; AnderSon, Erik ; AtTwood, David: Soft X-ray microscopy at a spatial resolution better than 15nm. In: Nature 435 (2005), S. $1210-1213$

[Chao u. a. 2009a] ChaO, Weilun; KIm, Jihoon ; Rekawa, Senajith ; Fischer, Peter ; Anderson, Erik: Demonstration of $12 \mathrm{~nm}$ Resolution Fresnel Zone Plate Lens based Soft X-ray Microscopy. In: Optics Express 17 (20) (2009), S. 17669 - 17677

[Chao u.a. 2009b] Chao, Weilun ; Kim, Jihoon ; Rekawa, Senajith ; Fischer, Peter ; Anderson, Erik: Hydrogen silsesquioxane double patterning process for 12 nm resolution x-ray zone plates. In: Journal of Vacuum Science 63 Technology B 27 (2009), S. $2606-2611$

[Chu u. a. 2008] Chu, Y. S. ; Yi, J. M. ; Carlo, F. D. ; Shen, Q. ; Lee, Wah-Keat ; Wu, H. J. ; Wang, C. L. ; Wang, J. Y. ; LiU, C. J. ; Wang, C. H. ; Wu, S. R. ; Chien, C. C. ; Hwu, Y. ; Tkachuk, A. ; Yun, W. ; Feser, M. ; Liang, K. S. ; Yang, C. S. ; Je, J. H. ; Margaritondo, G.: Hard-x-ray microscopy with Fresnel zone plates reaches $40 \mathrm{~nm}$ Rayleigh resolution. In: Applied Physics Letters 92 (2008), S. 103119

[Conley u. a. 2008] Conley, Ray ; Liu, Chian ; Qian, Jun ; Kewish, Cameron M. ; Macrander, Albert T. ; Yan, Hanfei ; Kang, Hyon C. ; Maser, Jörg ; StephenSon, G. B.: Wedged multilayer Laue lens. In: Review of Scientific Instruments 79 (2008), S. 053104

[Corkum 1993] Corkum, P. B.: Plasma Perspective on Strong-Field Multiphoton Ionization. In: Physical Review Letters 71 (1993), S. $1994-1997$ 
[Cross und Valentini 1982] Cross, Jon B. ; Valentini, James J.: High repetition rate pulsed nozzle beam source. In: Review of Scientific Instruments 53 (1982), S. 38 $-42$

[Daly 2009] DALY, Michael J.: A new perspective on radiation resistance based on Deinococcus radiodurans. In: Nature Reviews Microbiology 7 (2009), S. 237 - 245

[Derbenev u. a. 1982] Derbenev, Y. S. ; Kondratenko, A. M. ; Saldin, E. L.: On the Possibility of Using a Free Electron Laser For Polarization of Electrons in Storage Rings. In: Nuclear Instruments and Methods 193 (1982), S. 415 - 421

[Dierolf u. a. 2010] Dierolf, Martin ; Thibault, Pierre ; Menzel, Andreas ; Kewish, Cameron M. ; Jefimovs, Konstantins ; Schlichting, Ilme ; König, Konstanze von ; Bunk, Oliver ; Pfeiffer, Franz: Ptychographic coherent diffractive imaging of weakly scattering specimens. In: New Journal of Physics 12 (2010), S. 035017

[Egan 2006] EGAN, Patrick: fwhm. http://www.mathworks.com/matlabcentral/ fileexchange/10590-fwhm. März 2006

[Eisebitt u. a. 2004] Eisebitt, S. ; LÜning, J. ; Schlotter, W. F. ; Lörgen, M. ; Hellwig, O. ; Eberhardt, W. ; Stöhr, J.: Lensless imaging of magnetic nanostructures by X-ray spectro-holography. In: Nature 432 (2004), S. 885

[Even u. a. 2000] Even, U. ; Jortner, J. ; Noy, D. ; Lavie, N. ; Cossart-Magos, C.: Cooling of large molecules below $1 \mathrm{~K}$ and He clusters formation. In: Journal of Chemical Physics 112 (2000), S. 8068

[Faatz u. a. 2011] Faatz, B. ; Baboi, N. ; Ayvazyan, V. ; Balandin, V. ; Decking, W. ; Duesterer, S. ; Eckoldt, H.-J. ; Feldhaus, J. ; Golubeva, N. ; HonkavaAra, K. ; Koerfer, M. ; Laarmann, T. ; Leuschner, A. ; Lilje, L. ; LimBerg, T. ; Noelle, D. ; Obier, F. ; Petrov, A. ; Ploenjes, E. ; Rehlich, K. ; Schlarb, H. ; Schmidt, B. ; Schmitz, M. ; Schreiber, S. ; Schulte-Schrepping, H. ; Spengler, J. ; Staack, M. ; Tavella, F. ; Tiedtke, K. ; Tischer, M. ; Treusch, R. ; Vogt, M. ; Willner, A. ; Bahrdt, J. ; Follath, R. ; Gensch, M. ; Holldack, K. ; Meseck, A. ; Mitzner, R. ; Drescher, M. ; Miltchev, V. ; Rösch-Schulenburg, J. ; Rossbach, J.: Flash II: Perspectives and challenges. In: Nuclear Instruments and Methods in Physics Research Section A: Accelerators, Spectrometers, Detectors and Associated Equipment 635 (2011), S. 2 - 5

[Fiedorowicz u. a. 2001] Fiedorowicz, H. ; Bartnik, A. ; Dunn, J. ; Smith, R. F. ; Hunter, J. ; Nilsen, J. ; Osterheld, A. L. ; Shlyaptsev, V. N.: Demonstration of a neonlike argon soft-x-ray laser with a picosecond-laser-irradiated gas puff target. In: Optics Letters 26 (2001), S. $1403-1405$ 
[Fuhse 2006] Fuhse, Christian: X-ray waveguides and waveguide-based lensless imaging, Universität Göttingen, Dissertation, 2006

[Fuhse und Salditt 2006a] Fuhse, Christian ; SAlditt, Tim: Finite-difference field calculations for two-dimensionally confined x-ray waveguides. In: Applied Optics 45 (2006), S. $4603-4608$

[Fuhse und Salditt 2006b] Fuhse, Christian ; Salditt, Tim: Propagation of X-rays in ultra-narrow slits. In: Optics Communications 265 (2006), S. $140-146$

[Ghafoor u. a. 2008] Ghafoor, Naureen ; Eriksson, Fredrik ; Gullikson, Eric ; Hultman, Lars ; Birch, Jens: Incorporation of nitrogen in $\mathrm{Cr} / \mathrm{Sc}$ multilayers giving improved soft x-ray reflectivity. In: Applied Physics Letters (2008), S. 091913

[Giewekemeyer 2011] GIEWEKEMEYER, Klaus: A study on new approaches in coherent x-ray microscopy of biological specimens, Universität Göttingen, Dissertation, 2011

[Giewekemeyer u. a. 2010a] GieweKemeyer, Klaus ; Neubauer, Henrike ; KalbFleisch, Sebastian ; KrÜger, Sven P. ; SAlditt, Tim: Holographic and diffractive $\mathrm{x}$-ray imaging using waveguides as quasi-point sources. In: New Journal of Physics 12 (2010), S. 035008

[Giewekemeyer u.a. 2010b] Giewekemeyer, Klaus ; Thibault, Pierre ; Kalbfleisch, Sebastian ; Beerlink, Andre ; Kewish, C. M. ; Dierolf, M. ; Pfeiffer, Franz; SALDiTT, Tim: Quantitative biological imaging by ptychographic x-ray diffraction microscopy. In: Proceedings of the National Academy of Sciences 107 (2010), S. $529-534$

[Goldberg u. a. 1996] Goldberg, K. A. ; Tejnil, E. ; Bokor, J.: A 3-D Numerical Study of Pinhole Diffraction to Predict the Accuracy of EUV Point Diffraction Interferometry. In: Proceedings of Extreme Ultraviolet Lithography, Optical Society of America (1996), S. 142

[GPL 2007] GPL: GNU General Public License. Free Software Foundation, Genf, Schweiz, 2007

[Griem 2005] Griem, Hans R.: Principles of Plasma Spectroscopy. Cambridge University Press, 2005

[Großmann 2009] Grossmann, Peter: Absorptionsspektroskopische Messungen mit piezogesteuerter XUV-Laserplasmaquelle, Fachhochschule Hildesheim/Holzminden/Göttingen, Diplomarbeit, 2009 
[Großmann 2011] Grossmann, Peter: persönliche Mitteilung, zu veröffentlichendes Manuskript. 2011

[Guilbaud u. a. 2010] Guilbaud, O. ; Tissandier, F. ; Goddet, J-P. ; Ribière, M. ; Sebban, S. ; Gautier, J. ; Joyeux, D. ; Ros, D. ; Cassou, K. ; Kazamias, S. ; Klisnick, A. ; Habib, J. ; Zeitoun, P. ; Benredjem, D. ; Mocek, T. ; Nedjl, J. ; Rossi, S. de ; Maynard, G. ; Cros, B. ; BoudaA, A. ; Calisti, A.: Fourier-limited seeded soft x-ray laser pulse. In: Optics Letters 35 (2010), S. 1326 - 1329

[Guttmann u. a. 2003] Guttmann, P. ; Niemann, B. ; Rehbein, S. ; Knöchel, C. ; Rudolph, D. ; Schmahl, G.: The transmission X-ray microscope at BESSY II. In: Journal de Physique IV 104 (2003), S. 85 - 90

[Hayles u.a. 2009] Hayles, Mike ; Jiao, Chengge ; Lieftink, Hans ; Nemecek, Tomas ; Reyntuens, Steve ; Wall, David: Nova NanoLab 200/600 System Users Guide. 5350 NE Dawson Creek Drive Hillsboro, OR 97124: FEI Company (Veranst.), 2009

[Hell 2003] HeLl, Stefan W.: Jahrbuch der Max-Planck-Gesellschaft. Max-PlanckGesellschaft, 2003

[Henke u. a. 1993] Henke, B. L. ; Gullikson, E. M. ; DAvis, J. C.: X-ray interactions: photoabsorption, scattering, transmission, and reflection at $\mathrm{E}=50-30000 \mathrm{eV}, \mathrm{Z}=1-92$. In: Atomic Data and Nuclear Data Tables 54 (1993), S. $181-342$

[von Hofsten u. a. 2009] Hofsten, Olov von ; Bertilson, Michael ; Reinspach, Julia ; Holmberg, Anders ; Hertz, Hans M. ; Vogt, Ulrich: Sub-25-nm laboratory x-ray microscopy using a compound Fresnel zone plate. In: Optics Letters 37 (2009), S. $2631-2633$

[Hoppe 1969] Hoppe, Walter: Beugung im Inhomogenen Primärstrahlwellenfeld. I. Prinzip einer Phasenmessung von Elektronenbeugungsinterferenzen. In: Acta Crystallographica Section A 25 (1969), S. $495-501$

[ISO11146 1999] International Organisation for Standardization, Genf, SCHWEIZ: ISO/FDIS 11146: Laser and laser-related equipment - Test methods for laser beam parameters - Beam width, divergence angle and beam propagation factor. 1999

[ITT Visual Information Solutions ] ITT Visual Information Solutions: Interactive Data Language. http://www.ittvis.com/language/en-us/productsservices/ idl.aspx 
[J. W. Strutt, 3rd Baron Rayleigh 1878] J. W. Strutt, 3Rd Baron Rayleigh: On the Instability of Jets. In: Proceedings of the London Mathematical Society 10 (1878), S. $4-13$

[J. W. Strutt, 3rd Baron Rayleigh 1879] J. W. Strutt, 3Rd Baron Rayleigh: Investigations in Optics, with special reference to the Spectroscope. In: Philosophical Magazine Series 58 (1879), S. $261-274$

[Jansson u. a. 2005] Jansson, P. A. C. ; Vogt, U. ; Hertz, H. M.: Liquid-nitrogen-jet laserplasma source for compact soft x-ray microscopy. In: Revied Scientific Instruments 76 (2005), S. 43503

[Janulewicz u. a. 2004] Janulewicz, K. A. ; Lucianetti, A. ; Priebe, G. ; Nickles, P. V.: Review of state-of-the-art and output characteristics of table-top soft x-ray lasers. In: X-Ray Spectrometry 33 (2004), S. $262-266$

[Kalbfleisch 2006] Kalbfleisch, Sebastian: Fresnelsche Reflexionszonenplatten für harte Röntgenstrahlung, Universität Göttingen, Diplomarbeit, 2006

[Kamijo u. a. 2003] KamiJo, Nagao ; Suzuki, Yoshio ; TAkano, Hidekazu ; Tamura, Shigeharu ; Yasumoto, Masato ; Takeuchi, Akihisa ; Awaji, Mitsuhiro: Microbeam of $100 \mathrm{keV}$ x ray with a sputtered-sliced Fresnel zone plate. In: Review of Scientific Instruments 74 (2003), S. $5101-5104$

[Kang u. a. 2006] Kang, Hyon C. ; Maser, Jörg ; Stephenson, G. B. ; Liu, Chian ; Conley, Ray ; Macrander, Albert T. ; Vogt, Stefan: Nanometer Linear Focusing of Hard X Rays by a Multilayer Laue Lens. In: Physical Review Letters 96 (2006), S. 127401

[Kang u.a. 2007] Kang, Hyon C. ; Stephenson, G. B. ; Liu, Chian ; Conley, Ray ; Khachatryan, Ruben ; Wieczorek, Michael ; Macrander, Albert T. ; Yan, Hanfei ; Maser, Jörg ; Hiller, Jon ; Koritala, Rachel: Sectioning of multilayers to make a multilayer Laue lens. In: Review of Scientific Instruments 78 (2007), S. 046103

[Kang u.a. 2008] Kang, Hyon C. ; Yan, Hanfei ; Winarski, Robert P. ; Holt, Martin V. ; Maser, Jörg ; Liu, Chian ; Conley, Ray ; Vogt, Stefan ; Macrander, Albert T. ; Stephenson, G. B.: Focusing of hard x-rays to 16 nanometers with a multilayer Laue lens. In: Applied Physics Letters 92 (2008), S. 221114

[Kewish u. a. 2010] Kewish, C. M. ; Thibault, P. ; Dierolf, M. ; Bunk, O. ; Menzel, A. ; Vila-Comamala, J. ; Jefimovs, K. ; Pfeiffer, F.: Ptychographic characterization of the wavefield in the focus of reflective hard x-ray optics. In: Ultramicroscopy 110 (2010), S. $325-329$ 
[Kim 1989] KIM, Kwang-Je: Characteristics of Synchrotron Radiation. In: American Institute of Physics - Conference Proceedings (1989), S. 565-632

[Kirkpatrick und Baez 1948] Kirkpatrick, P. ; Baez, A. V.: Formation Of Optical Images By X-Rays. In: Optical Society Of America 9 (1948), S. 766 - 774

[Knaack u. a. 2004] KnaAck, S. A. ; Eddington, J. ; Leonard, Q. ; Cerrina, F. ; Onellion, M.: Dense arrays of nanopores as x-ray lithography masks. In: Applied Physics Letters 84 (2004), S. 3388 - 3390

[Kopylov u. a. 1995] Kopylov, Yuri V.; Popov, Alexei V. ; Vinogradov, Alexander V.: Application of the parabolic wave equation to X-ray diffraction optics. In: Optics Communications 118 (1995), S. 619 - 636

[Kopylov u. a. 1996] Kopylov, Yuri V.; Popov, Alexei V.; Vinogradov, Alexander V.: Diffraction phenomena inside thick Fresnel zone plates. In: Radio Science 31 (1996), S. $1815-1822$

[Koyama u.a. 2011a] Koyama, T. ; Takenaka, H. ; Ichimaru, S. ; Ohchi, T. ; Tsujia, T. ; Takanoa, H. ; Kagoshima, Y.: Development of Multilayer Laue Lenses; (1) Linear Type. In: American Institute of Physics - Conference Proceedings 1365 (2011), S. $24-27$

[Koyama u.a. 2011b] Koyama, T. ; Tsuji, T. ; Takano, H. ; Kagoshima, Y. ; Ichimaru, S. ; Ohchi, T. ; TAkenaka, H.: Development of Multilayer Laue Lenses; (2) Circular Type. In: American Institute of Physics - Conference Proceedings 1365 (2011), S. $100-103$

[Kranzusch u.a. 2003] Kranzusch, Sebastian ; Peth, Christian ; Mann, Klaus: Spatial characterization of extreme ultraviolet plasmas generated by laser excitation of xenon gas targets. In: Review of Scientific Instruments 74 (2003), S. 969 - 974

[Krebs 2011]ＫReBS, Hans-Ulrich: persönliche Mitteilung. Februar 2011

[Krebs und Bremert 1993] KreBs, Hans-Ulrich ; Bremert, Olaf: Pulsed laser deposition of thin metallic alloys. In: Applied Physics Letters 62 (1993), S. 2341

[Krieger 2001] KRIEger, Hanno: Strahlenphysik, Dosimetrie und Strahlenschutz, Band 2, Strahlungsquellen, Detektoren und klinische Dosimetrie. Teubner Verlag, 2001

[Kruer 1988] Kruen, William: The Physics of Laser Plasma Interactions. AddisonWesley, 1988 
[Kubiak u. a. 1995] Kubiak, G. D. ; Krenz, K. D. ; Berger, K. W. ; Trucano, T. G. ; Fisher, P. W. ; Gouge, M. J.: Cryogenic pellet laser plasma source targets. In: Optical Society of America Conference on Extreme Ultraviolet Lithography 23 (1995), S. 248

[Kurokhtin und Popov 2002] Kurokhtin, Alexei N. ; Popov, Alexei V.: Simulation of high-resolution x-ray zone plates. In: Journal of the Optical Society of America A 19 (2002), S. $315-324$

[Lammel 2003] LAMmEL, Oliver: Lineare und nichtlineare absorptions- und emissionsspektroskopische Charakterisierung eines LiF:F2--Farbzentrenkristalls, Universität Regensburg, Dissertation, 2003

[Lauterborn und Kurz 2003] Lauterborn, Werner ; Kurz, Thomas: Coherent Optics: fundamentals and applications. 2. Springer-Verlag, 2003

[Lübbecke und Schäfer] LüBbecke, Maik ; Schäfer, Bernd: Mr. Beam. LaserLaboratorium Göttingen e.V.. - URL http://www.llg-ev.de/abteilungen/ optik-kurze-wellenlaengen/hartmann-shack-wavefront-sensor.html. - LaserLaboratorium Göttingen e.V.

[Levin-Zaidman u. a. 2003] Levin-Zaidman, Smadar ; Englander, Joseph ; Shimoni, Eyal ; Sharma, Ajay K. ; Minton, Kenneth W. ; Minsky, Abraham: Ringlike Structure of the Deinococcus radiodurans Genome: A Key to Radioresistance? In: Science 299 (2003), S. $254-256$

[Liese u. a. 2010] Liese, Tobias ; RAdisch, Volker ; KreBs, Hans-Ulrich: Fabrication of multilayer Laue lenses by a combination of pulsed laser deposition and focused ion beam. In: Review of Scientific Instruments 81 (2010), S. 073710

[Liese u. a. 2011] Liese, Tobias ; RADisch, Volker ; KnorR, Ing ; ReEse, Michael ; Grossmann, Peter ; Mann, Klaus ; Krebs, Hans-Ulrich: Development of laser deposited multilayer zone plate structures for soft X-ray radiation. In: Applied Surface Science 257 (2011), S. $5138-5141$

[Lim u. a. 2011] Lim, Jun ; Shin, Hyun J. ; Hong, Chung K.: High-Resolution Soft Xray Digital In-Line Holographic Microscopy. In: Japanese Journal of Applied Physics 50 (2011), S. 072504

[MacDonald und Gibson 2003] MacDonald, C. A. ; Gibson, W. M.: Applications and advances in polycapillary optics. In: X-Ray Spectrometry 3 (2003), S. $258-268$ 
[Maiden und Rodenburg 2009] Maiden, A. M. ; Rodenburg, J. M.: An improved ptychographical phase retrieval algorithm for diffractive imaging. In: Ultramicroscopy 109 (2009), S. $1256-1262$

[Mancuso u. a. 2010] Mancuso, A. P. ; Gorniak, Th. ; Staier, F. ; Yefanov, O. M. ; Barth, R. ; Christophis, C. ; Reime, B. ; Gulden, J. ; Singer, A. ; Pettit, M. E. ; Nisius, Th. ; Wilhein, Th. ; Gutt, C. ; Grübel, G. ; Guerassimova, N. ; Treusch, R. ; Feldhaus, J. ; Eisebitt, S. ; Weckert, E. ; Grunze, M. ; Rosenhahn, A. ; Vartanyants, I. A.: Coherent imaging of biological samples with femtosecond pulses at the free-electron laser FLASH. In: New Journal of Physics 12 (2010), S. 035003

[MathWorks Inc. 2009] MathWorks InC.: MatLab 2009a. http://www.mathworks . $\mathrm{de} /$ products/matlab/. 2009

[Mayer-Kuckuk 1997] Mayer-Kuckuk, Theo: Atomphysik. Teubner Studienbücher, 1997

[McCarthy und Molloy 1974] McCarthy, M. J. ; Molloy, N. A.: Review of stability of liquid jets and the influence of nozzle design. In: The Chemical Engineering Journal 7 (1974), S. $1-20$

[Morgan 1975] Morgan, C. G.: Laser-induced breakdown of gases. In: Reports On Progress in Physics 38 (1975), S. $612-665$

[Murphy und Pellegrini 1985] Murphy, J. B. ; Pellegrini, C.: Generation of highintensity coherent radiation in the soft-x-ray and vacuum-ultraviolet region. In: Journal of the Optical Society of America B 2 (1985), S. 259 - 264

[Nugent-Glandorf u. a. 2002] Nugent-Glandorf, L. ; Scheer, M. ; Samuels, D. A. ; Bierbaum, V. ; Leone, S. R.: A laser based instrument fort he study of ultrafast chemical dynamics by soft x-ray-probe photoelectron spectroscopy. In: $R e$ view of Scientific Instruments 73 (2002), S. 1875 - 1886

[Osterhoff und Salditt 2009] Osterhoff, Markus ; Salditt, Tim: Real structure effects in X-ray waveguide optics: The influence of interfacial roughness and refractive index profile on the near-field and far-field distribution. In: Optics Communications 282 (2009), S. $3250-3256$

[Paganin 2006] Paganin, D. M.: Coherent X-Ray Optics. Oxford University Press, 2006 
[Peth 2008] Peth, Christian: XUV-Laserplasmaquellen für die AbsorptionsSpektroskopie und zeitaufgelöste Röntgenbeugung, Universität Göttingen, Dissertation, 2008

[Peth u. a. 2007] Peth, Christian ; Kalinin, Anton ; Barkusky, Frank ; Mann, Klaus ; Toennies, J. P. ; Rusin, Lev Y.: XUV laser-plasma source based on solid Ar filament. In: Review of Scientific Instruments 78 (2007), S. 103509

[Pezzagna u.a. 2010] Pezzagna, Sébastien ; Wildanger, Dominik ; Mazarov, Paul ; Wieck, Andreas D. ; Sarov, Yanko ; Rangelow, Ivo ; Naydenov, Boris ; Jelezko, Fedor ; Hell, Stefan W. ; Meijer, Jan: Nanoscale Engineering and Optical Addressing of Single Spins in Diamond. In: Small 6 (2010), S. 2117 - 2121

[Proch und Trickl 1989] Proch, D. ; TrickL, T.: A high-intensity multi-purpose piezo-electric pulsed molecular beam source. In: Revied Scientific Instruments 60 (1989), S. $713-716$

[Quitmann u. a. 2009] Quitmann, Christoph ; David, Christian ; Nolting, Frithjof ; Pfeiffer, Franz ; Stampanoni, Marco: 9th International Conference on X-Ray Microscopy. In: Journal of Physics: Conference Series 186 (2009), S. 011001

[R Development Core Team 2011] R Development Core Team: R: A Language and Environment for Statistical Computing. Vienna, Austria: R Foundation for Statistical Computing (Veranst.), 2011. - URL http://www.R-project.org/

[Rasband 1997 - 2011] RASBAND, W.S.: ImageJ. http://imagej.nih.gov/ij/. 1997 $-2011$

[Röder 2009] RöDER, Johanna: Analyse der Glättung rauer Oberflächen durch Dünnschichtendeposition, Universität Göttingen, Dissertation, 2009

[Röder u. a. 2010] RöDER, Johanna ; Liese, Tobias ; KREBs, Hans-Ulrich: Materialdependent smoothing of periodic rippled structures by pulsed laser deposition. In: Journal of Applied Physics 107 (2010), S. 103515

[Reale u. a. 2008] Reale, Lucia ; Bonfigli, Francesca ; Lai, Antonia ; Flora, Francesco ; Poma, Anna ; Albertano, Patrizia ; Bellezza, Simona ; Montereali, Rosa M. ; Faenov, Anatoly ; Pikuz, Tania ; Almaviva, Salvatore ; Vincenti, Maria A. ; Francucci, Massimo ; Gaudio, Pasqualino ; Martellucci, Sergio ; RiChetTA, Maria: X-ray Microscopy of Plant Cells by Using LiF Crystal as a Detector. In: Microscopy Research and Technique 71 (2008), S. $839-848$ 
[Reese u. a. 2011] Reese, Michael ; Schäfer, Bernd ; Grossmann, Peter ; Bayer, Armin ; Mann, Klaus ; Liese, Tobias ; KreBs, Hans-Ulrich: Submicron focusing of XUV radiation from a laser plasma source using a multilayer Laue lens. In: Applied Physics A 102 (2011), S. 85-90

[Röntgen 1896] Röntgen, Wilhelm C.: Über eine neue Art von Strahlen. In: Sonderabbdruck aus den Sitzungsberichten der Würzburger physikalisch-medizinischen Gesellschaft zu Würzburg (1896), S. $1-22$

[Rodenburg und Faulkner 2004] Rodenburg, J. M. ; Faulkner, H. M. L.: A phase retrieval algorithm for shifting illumination. In: Applied Physics Letters 85 (2004), S. $4795-4797$

[Rodenburg u. a. 2007] Rodenburg, J. M. ; Hurst, A. C. ; Cullis, A. G. ; Dobson, B. R. ; Pfeiffer, F. ; Bunk, O. ; David, C. ; Jefimovs, K. ; Johnson, I.: HardX-Ray Lensless Imaging of Extended Objects. In: Physical Review Letters 98 (2007), S. 034801

[Sakdinawat und Attwood 2010] Sakdinawat, Anne ; AtTwood, David: Nanoscale X-ray imaging. In: Nature Photonics (2010), S. $840-848$

[Saleh und Teich 2008] Saleh, Bahaa E. A. ; TEICH, Malvin C.: Fundamentals of Photonics, 2nd Edition. Wiley-VCH Verlag, 2008

[Sandberg u. a. 2007] SAndberg, Richard L. ; Paul, Ariel ; Raymondson, Daisy A. ; Hadrich, Steffen ; Gaudiosi, David M. ; Holtsnider, Jim ; Tobey, Ra'anan I. ; Cohen, Oren ; Murnane, Margaret M. ; Kapteyn, Henry C. ; Song, Changyong ; MiaO, Jianwei ; Liu, Yanwei ; Salmassi, Farhad: Lensless Diffractive Imaging Using Tabletop Coherent High-Harmonic Soft-X-Ray Beams. In: Physical Review Letters 99 (2007), Aug, S. 098103. - URL http://Iink.aps.org/doi/10.1103/PhysRevLett. 99.098103

[Sannomiya u. a. 2011] Sannomiya, Takumi ; Scholder, Olivier ; Jefimovs, Konstantins ; Hafner, Christian ; Dahlin, Andreas B.: Investigation of Plasmon Resonances in Metal Films with Nanohole Arrays for Biosensing Applications. In: Small 12 (2011), S. $1653-1663$

[Schäfers u.a. 1998] Schäfers, Franz ; Mertins, Hans-Christoph ; Schmolla, Frank ; Packe, Ingo ; Salashchenko, Nikolay N. ; Shamov, Eugeny A.: CrySc multilayers for the soft-x-ray range. In: Applied Optics 37 (1998), S. $719-728$

[Schlittgen 1996] Schlittgen, Rainer: Statistische Inferenz. Oldenbourg, 1996 
[Schlömer 2008] SCHLÖMER, Nico: Matlab2tikz. http://www.mathworks.com/ matlabcentral/fileexchange/22022-matlab2tikz. November 2008

[Schropp u. a. 2010] Schropp, A. ; Boye, P. ; Feldkamp, J. M. ; Hoppe, R. ; Patommel, J. ; Samberg, D. ; Stephan, S. ; Giewekemeyer, K. ; Wilke, R. N. ; Salditt, T. ; Gulden, J. ; Mancuso, A. P. ; Vartanyants, I. A. ; Weckert, E. ; Schoder, S. ; Burghammer, M. ; Schroer, C. G.: Hard x-ray nanobeam characterization by coherent diffraction microscopy. In: Applied Physics Letters 96 (2010), S. 091102

[Seres u. a. 2005] Seres, J. ; Seres, E. ; Verhoef, A. J. ; Tempea, G. ; Strelill, C. ; Wobrauschek, P. ; Yakovlev, V. ; Scrinzi, A. ; Spielmann, C. ; Krausz, F.: Source of coherent kiloelectronvolt X-rays. In: Nature 433 (2005), S. 596

[Skoglund u. a. 2010] Skoglund, P. ; Lundström, U. ; Vogt, U. ; Hertz, H. M.: High-brightness water-window electron-impact liquid-jet microfocus source. In: Applied Physics Letters 96 (2010), S. 084103

[Sommerer u. a. 1998] Sommerer, G. ; Mevel, E. ; Hollandt, J. ; Schulze, D. ; Nickles, P. V. ; Ulm, G. ; Sandner, W.: Absolute photon number measurement of high-order harmonics in the extreme UV. In: Optics Communications 146 (1998), S. $347-355$

[StatSoft, Inc. 2011] StatSoft, Inc.: Statistica 9.1. Tulsa, OK, USA: StatSoft, Inc. (Veranst.), 2011. - URL http://www.statsoft.com

[Takenaka u. a. 2009] Takenaka, H. ; Ichimaru, S. ; Ohchi, T. ; Koyama, T. ; Tsuji, T. ; Takano, H. ; Kagoshima, Y.: Mo/Si and MoSi2/Si nanostructures for multilayer Laue lens. In: Journal of Physics: Conference Series 186 (2009), S. 012074

[Takman u. a. 2007] Takman, P. A. C. ; Stollberg, H. ; Johansson, G. A. ; Holmberg, A. ; Lindblom, M. ; Hertz, H. M.: High-resolution compact X-ray microscopy. In: Journal of Microscopy 226 (2007), S. 175 - 181

[Tamura u.a. 2009] Tamura, Shigeharu ; Yasumoto, Masato ; KamiJo, Nagao ; Uesugi, K ; Takeuchi, Akihisa ; Terada, Y. ; Suzuki, Yoshio: Quasi-kinoform Type Multilayer Zone Plate with High Diffraction Efficiency for High-energy X-rays. In: Journal of Physics: Conference Series 186 (2009), S. 012075

[Tamura u. a. 2002] Tamura, Shigeharu ; Yasumoto, Masato ; Minara, Toshiyuki ; Kamiso, Nagao ; Suzuki, Yoshio ; AwajI, Mitsuhiro ; Takeuchi, Akihisa ; Takano, Hidekazu ; HANDA, Katsumi: Multilayer Fresnel zone plate for high-energy X-ray by DC sputtering deposition. In: Vacuum 66 (2002), S. 495 - 499 
[Thibault u. a. 2008] Thibault, Pierre ; Dierolf, Martin ; Menzel, Andreas ; Bunk, Oliver ; DAvid, Christian ; Pfeiffer, Franz: High-Resolution Scanning X-ray Diffraction Microscopy. In: Science 321 (2008), S. $379-382$

[Thieme u. a. 1998] Thieme, Jürgen ; Schmahl, Günter ; Rudolph, Dietbert: X-ray microscopy and spectromicroscopy. Springer-Verlag, 1998

[Tomassetti u. a. 2003] Tomassetti, G. ; Ritucci, A. ; Reale, A. ; Palladino, L. ; Reale, L. ; Arrizza, L. ; Baldacchini, G. ; Bonfigli, F. ; Flora, F. ; Mezi, L. ; Montereali, R. M. ; Kukhlevsky, S. V. ; Faenov, A. ; Pikuz, T. ; Kaiser, J.: High-resolution imaging of a soft-X-ray laser beam by color centers excitation in lithium fluoride crystals. In: Europhysics Letters 63 (2003), S. 681 - 686

[Töttger 2007] TöTTGER, Holger: Charakterisierung von Gas-Targets mittels Wellenfrontsensorik zur Optimierung laserproduzierter EUV-Plasmen, Fachhochschule Hildesheim/Holzminden/Göttingen, Diplomarbeit, 2007

[Vila-Comamala u. a. 2010] Vila-Comamala, Joan ; Gorelick, Sergey ; FÄrm, Elina ; Kewish, Cameron M. ; Diaz, Ana ; Barrett, Ray ; Guzenko, Vitaliy A. ; Ritala, Mikko ; David, Christian: Ultra-high resolution zone-doubled diffractive X-ray optics for the multi-keV regime. In: Optics Express 19 (2010), S. 175 - 184

[Vinogradov und Mitrofanow 2009] Vinogradov, Alexander V. ; Mitrofanow, A. N.: Study of Spatial Resolution of Circular and Off-Axis Zone Plates. In: Bulletin of the Lebedev Physics Institute 36 (2009), S. 263 - 269

[Wachulak u. a. 2010] Wachulak, P. W. ; Bartnik, A. ; Fiedorowicz, H. ; Feigl, T. ; Jarocki, R. ; Kostecki, J. ; Rakowski, R. ; Rudawski, P. ; Sawicka, M. ; SzczureK, M. ; SzczureK, A. ; ZaWADZKI, Z.: A compact, quasi-monochromatic laser-plasma EUV source based on a double-stream gas-puff target at $13.8 \mathrm{~nm}$ wavelength. In: Applied Physics B 100 (2010), S. $461-469$

[Wagenaars 2006] WagenaARs, Paul: Matfig2PGF. http://www.mathworks.com/ matlabcentral/fileexchange/12962. November 2006

[Wang 2003] WANG, Lei: Envelope 1.1. http://www.mathworks.com/matlabcentral/ fileexchange/3142-envelope1-1. März 2003

[Wang u. a. 2006] Wang, Y. ; Granados, E. ; Larotonda, M. A. ; Berrill, M. ; Luther, B. M. ; Patel, D. ; Menoni, C. S. ; Rocca, J. J.: High-Brightness InjectionSeeded Soft-X-Ray-Laser Amplifier Using a Solid Target. In: Physical Review Letters 97 (2006), S. 123901 
[Wolter 1952] Wolter, Hans: Spiegelsysteme streifenden Einfalls als abbildende Optiken für Röntgenstrahlung. In: Annalen der Physik 10 (1952), S. $94-114$

[Yin u.a. 2006] Yin, Gung-Chian ; Song, Yen-Fang ; Tang, Mau-Tsu ; Chen, FuRong ; Liang, Keng S. ; Duewer, Frederick W. ; Feser, Michael ; Yun, Wenbing ; SHIEH, Han-Ping D.: $30 \mathrm{~nm}$ resolution x-ray imaging at $8 \mathrm{keV}$ using third order diffraction of a zone plate lens objective in a transmission microscope. In: Applied Physics Letters 89 (2006), S. 221122 


\section{Danksagung}

Abschließend möchte ich all jenen danken, die mich bei der Entstehung dieser Arbeit unterstützten.

Ich danke Prof. Dr. Tim Salditt für die akademische Betreuung, die stete Gesprächsbereitschaft und seine Unterstützung sowie für das Erstreferat.

Dem Korreferenten Prof. Dr. Gerd Marowsky und Dr. Klaus Mann danke ich für den Themenvorschlag und die Möglichkeit, diese Arbeit am Laser-Laboratorium Göttingen e.V. anzufertigen.

Dr. Christian Peth und Dr. Anton Kalinin danke ich für die Einarbeitung und die Zusammenarbeit am Laborröntgenmikroskop. Prof. Dr. Hans-Ulrich Krebs, Tobias Liese und Volker Radisch möchte für die Herstellung der Multischicht-Laue-Linsen und für die Kooperation bei der Charakterisierung danken. Der Arbeitsgruppe PD Dr. Axel Rosenhahn, Dr. Mike Beckers, Tobias Senkbeil und Thomas Gorniak danke ich für die Messzeiten am BESSY II Speichering und Dr. Klaus Giewekemeyer, Dr. Dong-Du Mai, Markus Osterhoff, Dr. Sven-Philip Krüger und Henrike Neubauer für die Unterstützung bei der Auswertung der Daten und Simulation der ultra-kleinen Lochblenden.

Mein besonderer Dank für die stete freundschaftliche, moralische und fachliche Unterstützung im Entstehen dieser Arbeit, die wertvollen Gespräche und Diskussionen und das Lektorat dieser Arbeit gelten Dr. Katharina Lange, Dr. Klaus Giewekemeyer und Dr. Stephanie Kahle.

Des Weiteren danke ich

- meiner Arbeitsgruppe und allen Mitarbeitern des Laser-Laboratoriums für die freundschaftliche und humorvolle Zusammenarbeit,

- Jens-Oliver Dette, Alexander Göhmann, Dr. Bernd Schäfer, Dr. Armin Bayer, Stefan Döring, Maik Lübbeke, Andreas Spata, Olaf Mädiger und Dirk Born für die schnelle und kompetente Unterstützung in Konstruktions-, Physik-, Elektronik-, Computer-, Programmier- und Mechanikfragen,

- Jochen Herbst, Dr. Martin Röver und Christian Denker für die Unterstützung bei der Arbeit an den Geräten der Universität und die Herstellung von Proben und Filtern,

- Dr. Claudia Geisler und Dr. Dominik Wildanger für die gemeinsame Arbeit bei der Farbzentrenuntersuchung, 
- der DFG und den BMBF für die finanzielle Unterstützung im Rahmen des Sonderforschungsbereiches 755 „Nanoscale Photonic Imaging“ und des Projektes „Labratory X-ray Microscope“,

- den weiteren Lektoren meiner Arbeit Dr. Frank Barkusky, Julia Sedlmayer, Peter Großmann, Gabriele Reese und Janina Wolf von der Sahl.

Schließlich möchte ich insbesondere meiner Familie und meinen Freunden meinen Dank aussprechen, deren Unterstützung stets von unschätzbarem Wert für mich war. 